\title{
RESOURCE RECOVERY BY OSMOTIC BIOELECTROCHEMICAL SYSTEMS TOWARDS SUSTAINABLE WASTEWATER TREATMENT
}

\author{
Mohan Qin \\ Dissertation Submitted to the faculty of the Virginia Polytechnic Institute and State University in \\ partial fulfillment of the requirements for the degree of \\ Doctor of Philosophy in Civil Engineering
}

Zhen He, Chair

Andrea M. Dietrich

Daniel M. Gallagher

Amanda J. Morris

Oct. $5^{\text {th }}, 2017$

Blacksburg, VA

Keywords: Osmotic bioelectrochemical systems, resource recovery, bioelectrochemical systems, forward osmosis, wastewater. 


\title{
Resource Recovery by Osmotic Bioelectrochemical Systems towards Sustainable Wastewater Treatment
}

\author{
Mohan Qin
}

\begin{abstract}
(academic)
Recovering valuable resources from wastewater will transform wastewater management from a treatment focused to sustainability focused strategy, and creates the need for new technology development. An innovative treatment concept - osmotic bioelectrochemical system (OsBES), which is based on cooperation between bioelectrochemical systems (BES) and forward osmosis (FO), has been introduced and studied in the past few years. An OsBES can accomplish simultaneous treatment of wastewater and recovery of resources such as nutrient, energy, and water (NEW). The cooperation can be accomplished in either an integrated (osmotic microbial fuel cells, OsMFC) or coupled (microbial electrolysis cell-forward osmosis system, MEC-FO) configuration. In OsMFC, higher current generation than regular microbial fuel cell (MFC) was observed, resulting from the lower resistance of FO membrane. The electricity generation in OsMFC could greatly inhibit the reverse salt flux. Besides, ammonium removal was successfully demonstrated in OsMFC, making OsMFCs a promising technology for "NEW recovery" (NEW: nutrient, energy and water). For the coupled OsBES, an MEC-FO system was developed. The MEC produced an ammonium bicarbonate draw solute via recovering ammonia from synthetic organic solution, which was then applied in the FO for extracting water from the MEC anode effluent. The system has been advanced with treating landfill leachate. A mathematical model developed for ammonia removal/recovery in BES quantitatively confirmed that the $\mathrm{NH}_{4}{ }^{+}$ions serve as effective proton shuttles across cation exchange membrane (CEM).
\end{abstract}




\title{
Resource Recovery by Osmotic Bioelectrochemical Systems towards Sustainable Wastewater Treatment
}

\author{
Mohan Qin
}

\section{Abstract (general audience)}

Nowadays, wastewater is no longer considered as waste. Instead, it is a pool for different kinds of resources, such as nutrient, energy, and water (NEW). Various technologies were developed to achieve NEW recovery from wastewater. A novel concept, osmotic bioelectrochemical system (OsBES) has been introduced and studied in the past few years. OsBES is based on two technologies: bioelectrochemical systems (BES) and forward osmosis (FO); and the corporation between these two technologies could accomplish simultaneous wastewater treatment and resource recovery. We investigated two kinds of OsBES: one is osmotic microbial fuel cells (OsMFC), and the other is microbial electrolysis cell-forward osmosis system (MEC-FO). For OsMFC, a mathematical model was built to understand the internal resistance, which will affect the current generation according to Om's law $(\mathrm{I}=\mathrm{U} / \mathrm{R})$. The salt transport across the cation exchange membrane (CEM) is related to the current generation. The ion transport, especially ammonium/ammonia transport, across CEM membrane in BES was modelled, which will help the BES design and operation for ammonia recovery systems. The system performance for wastewater treatment and resource recovery in MEC-FO was fully investigated with both synthetic wastewater and landfill leachate. The results indicated that MEC-FO is a promising system for NEW recovery. 


\section{Acknowledgements}

First and foremost I thank my advisor, Dr. Zhen (Jason) He, for his guidance and mentorship these past four years. He has been more than an adviser, and I have benefited from his mentoring, patience, and encouragement. Thank you for offering me such a great opportunity of being an EBBLer and doing interesting research in bioelectrochemical systems. I sincerely thank my committee members: Dr. Andrea M. Dietrich, Dr. Daniel M. Gallagher, and Dr. Amanda J. Morris, for their insightful comments, thought-provoking questions and their support for me.

I would like to thank all of the EBBL members for their support and assistance during my research journey. Thanks to my collaborators, Dr. Ying Liu, Dr. Rui Qiao, Dr. William Maza, and Dr. Amanda Morris, for their great work.

I would also like to thank the Qatar National Research Fund (6-289-2-125) and Institute for Critical Technology and Applied Science (ICTAS) at Virginia Tech to financially support my research. Many thanks to Peppers Ferry Regional Wastewater Treatment Plant for providing inocula for my reactors.

Thanks to my family and friends. I wouldn't be able to accomplish anything in my life without their love and support. Finally to my husband, Haoran. You give me love and strength, and you are always there for me. 


\section{Table of Contents}

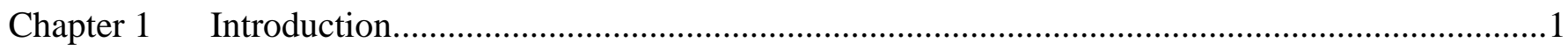

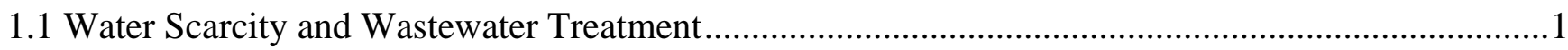

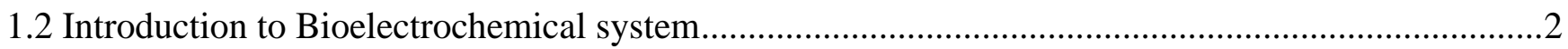

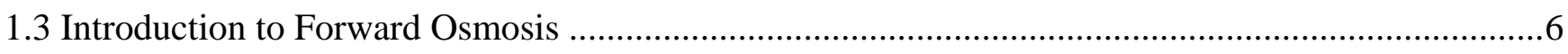

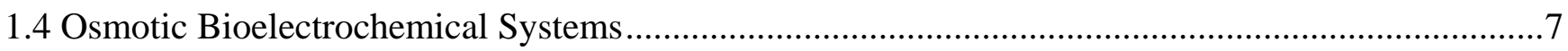

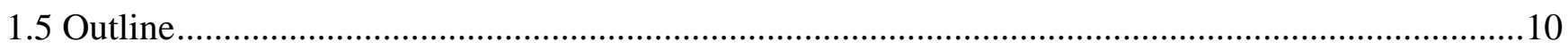

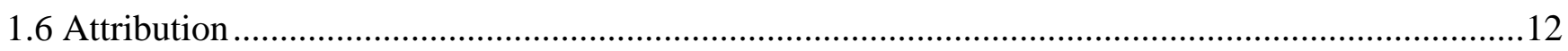

Chapter 2 Resource Recovery by Osmotic Bioelectrochemical Systems towards Sustainable

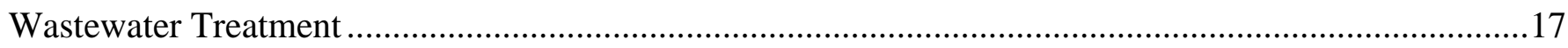

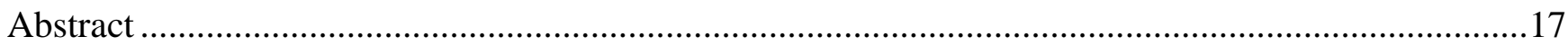

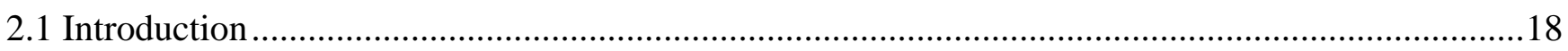

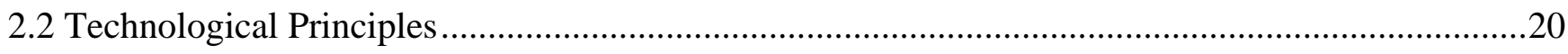

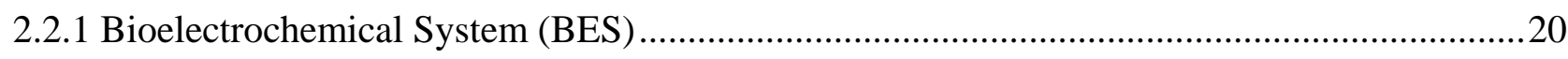

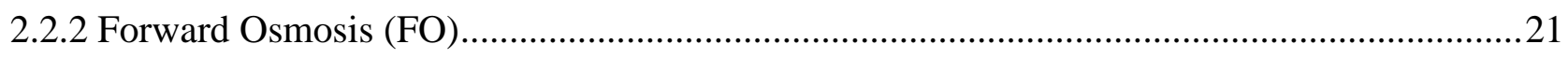

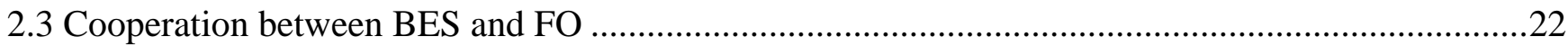

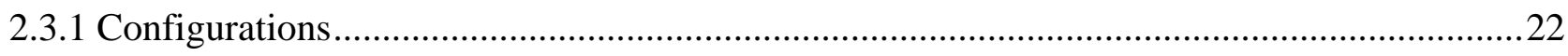

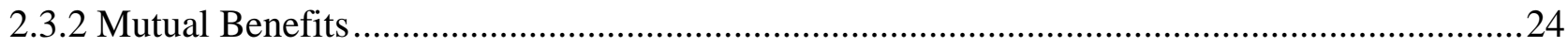

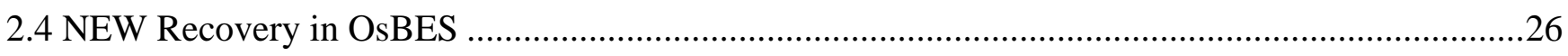

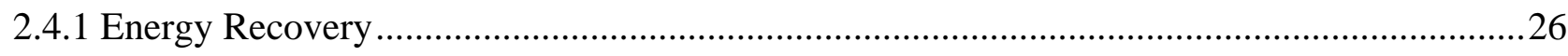

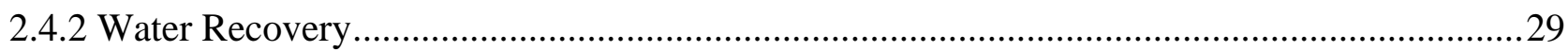

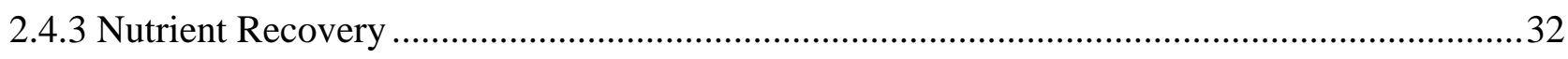

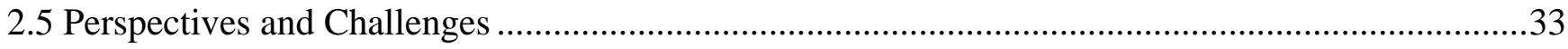

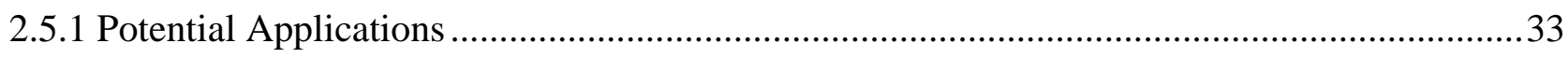

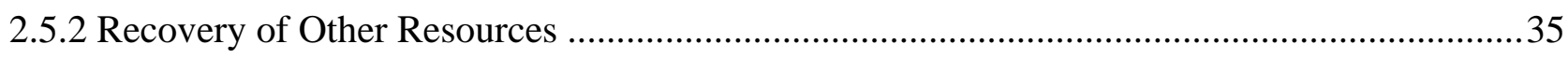

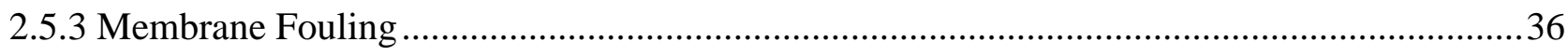

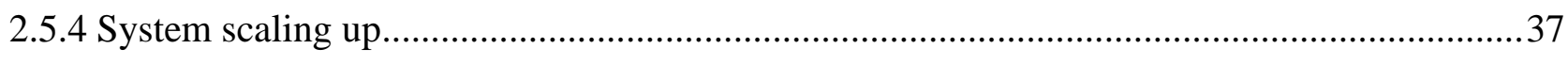

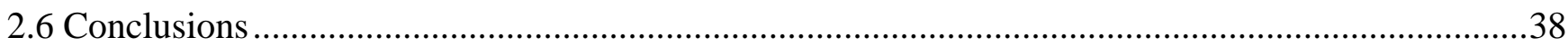

Chapter 3 Understanding Electricity Generation in Osmotic Microbial Fuel Cells through Integrated

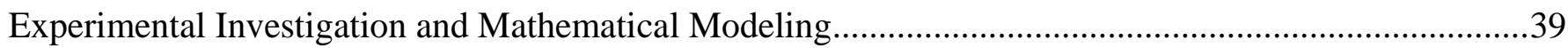

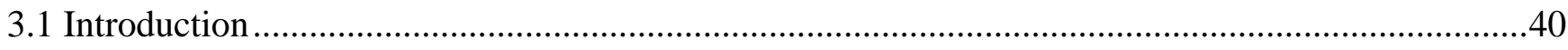

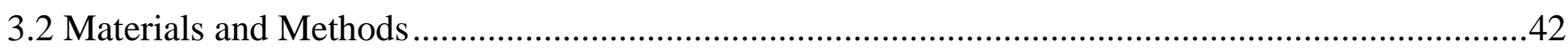

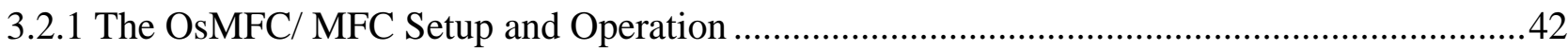

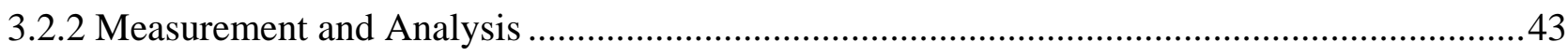

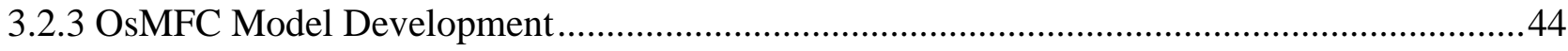

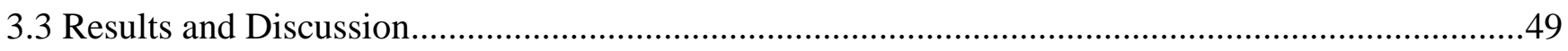

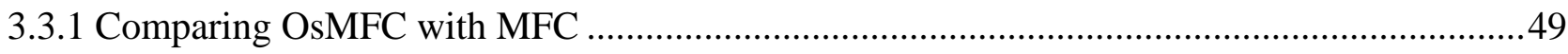

3.3.2 Effects of membrane and solution resistance....................................................................52

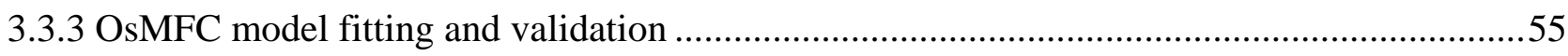

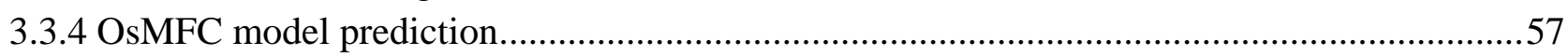

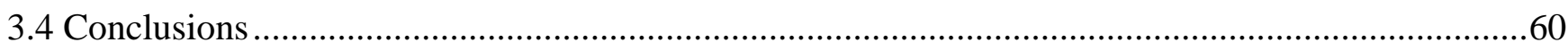


Chapter 4 Effects of Current Generation and Electrolyte $\mathrm{pH}$ on Reverse Salt Flux across Thin Film Composite Membrane in Osmotic Microbial Fuel Cells

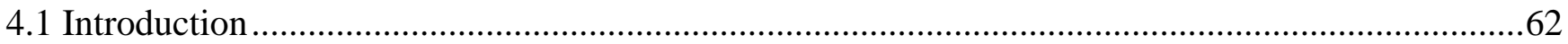

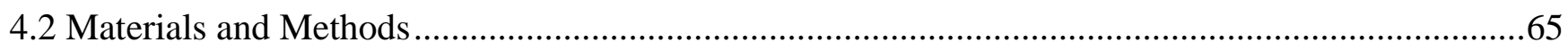

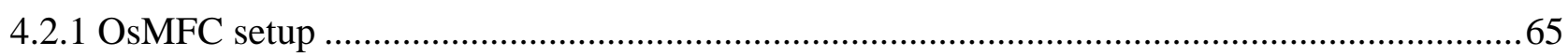

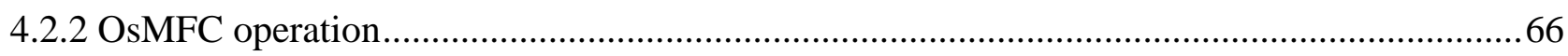

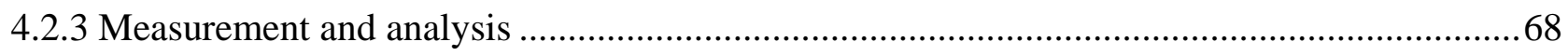

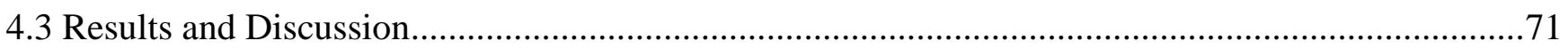

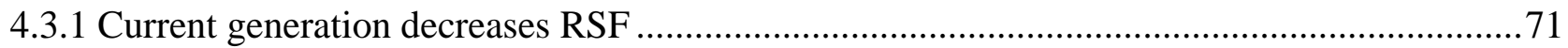

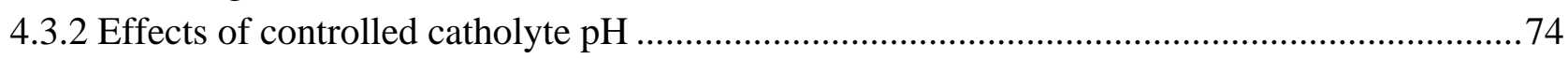

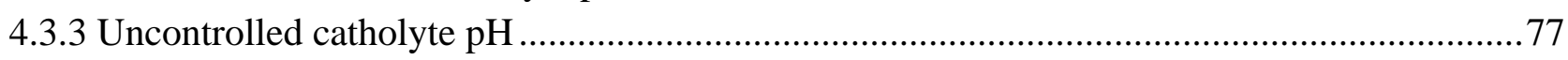

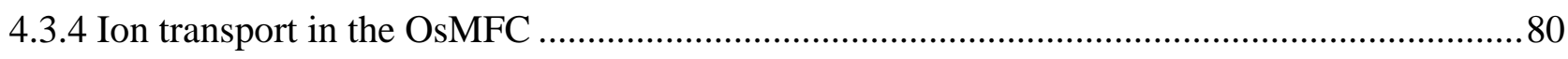

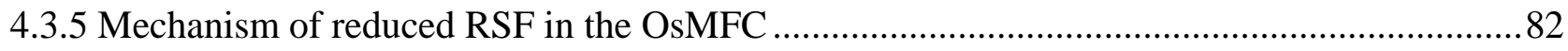

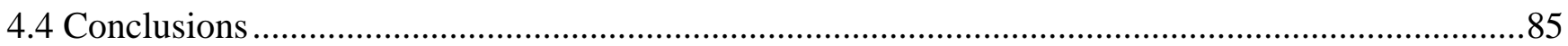

Chapter 5 Ammonium removal from synthetic wastewater promoted by current generation and water

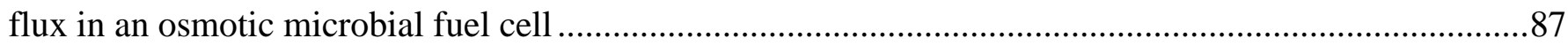

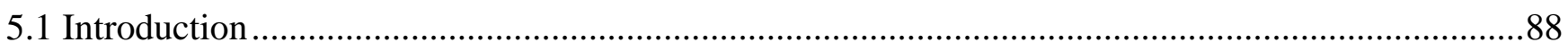

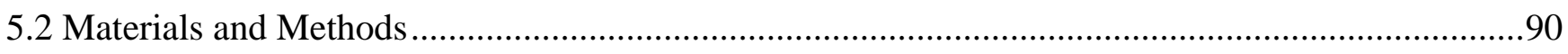

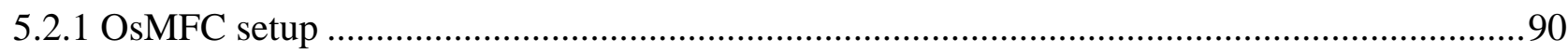

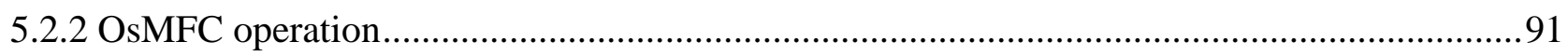

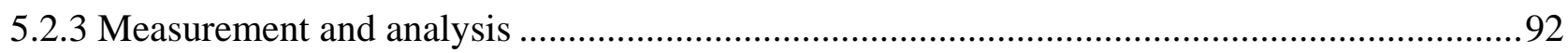

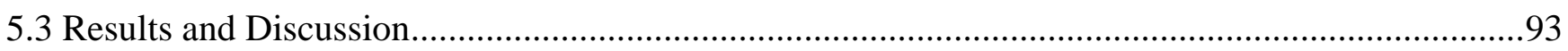

5.3.1 Feasibility of ammonium removal across FO membrane .................................................93

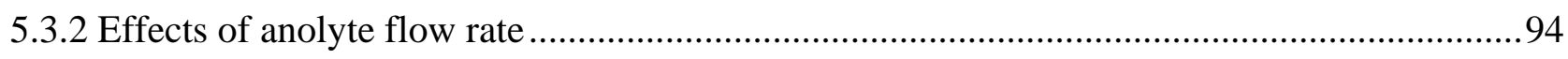

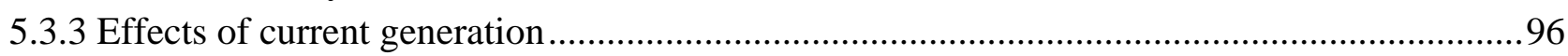

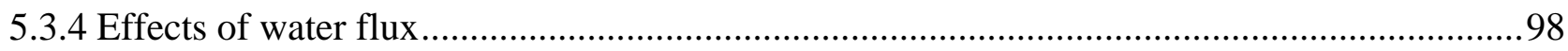

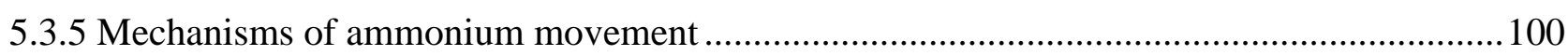

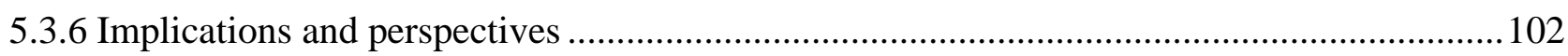

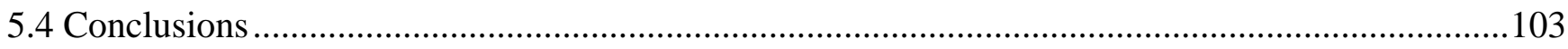

Chapter 6 Understanding Ammonium Transport in Bioelectrochemical Systems towards its Recovery 104

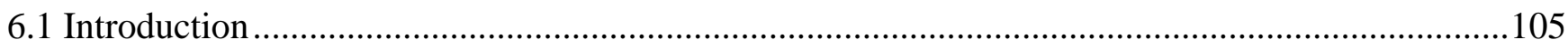

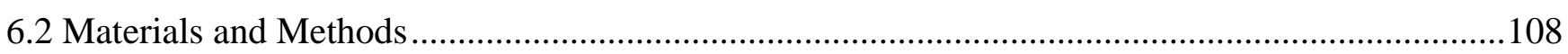

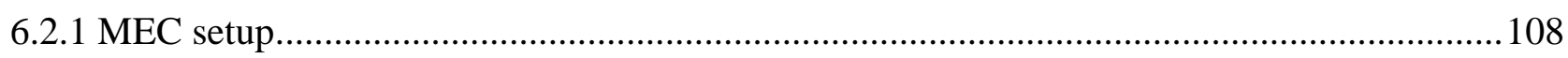

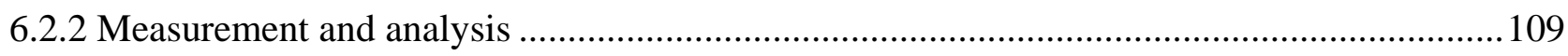

6.2.3 Mathematical model of BES-based ammonia recovery .............................................. 110

6.2.4 Modeling of electrochemical and chemical reactions in the MEC ...................................... 116

6.2.5 Governing equation for $\mathrm{H}^{+}$ion concentration in the anolyte .......................................... 117

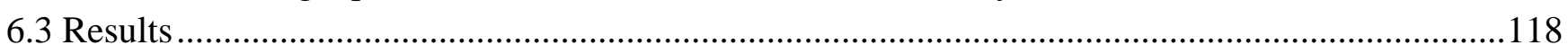

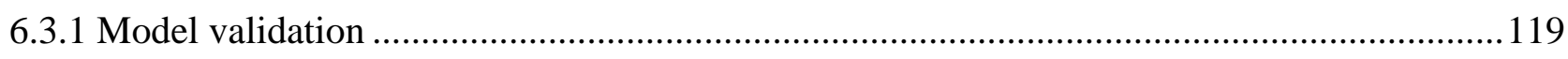

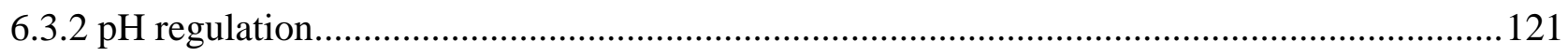

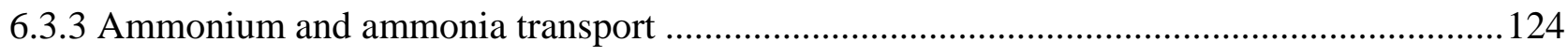

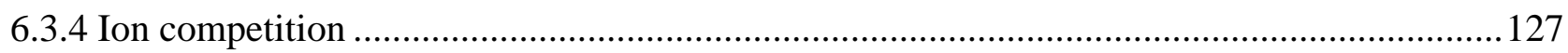

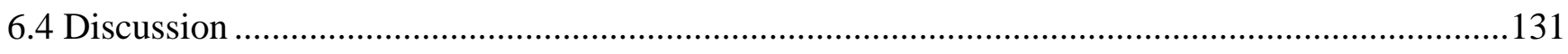


Chapter 7 Integrated Experimental and Modeling Evaluation of Energy Consumption for Ammonia

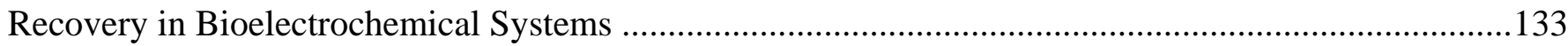

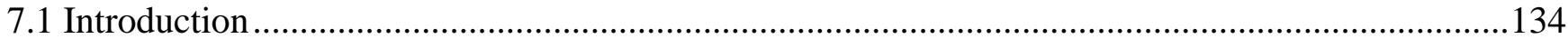

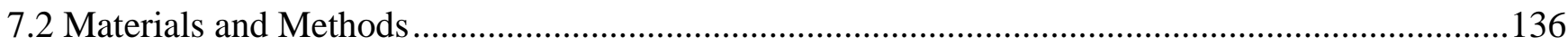

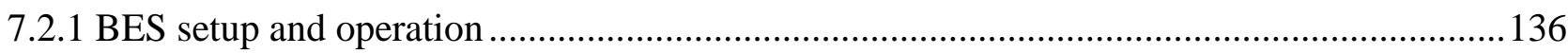

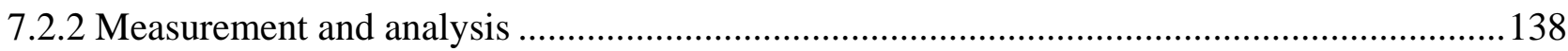

7.2.3 Mathematical model of BES-based ammonia recovery ..................................................... 140

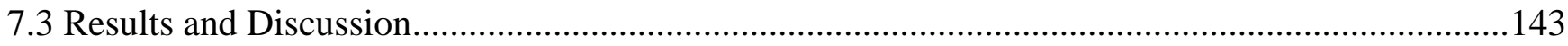

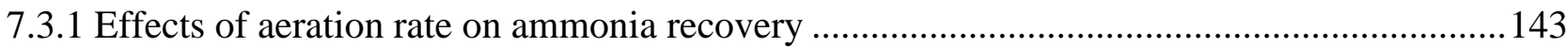

7.3.2 Effects of current generation - external voltage ............................................................ 148

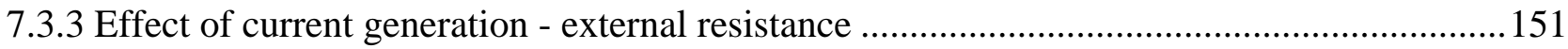

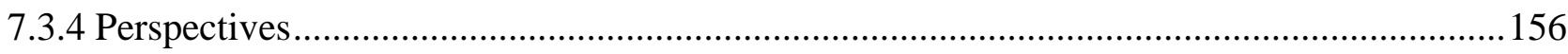

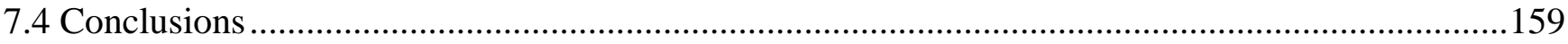

Chapter 8 Self-supplied ammonium bicarbonate draw solute for achieving wastewater treatment and recovery in a microbial electrolysis cell-forward osmosis-coupled system .........................................160

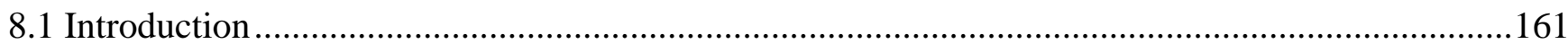

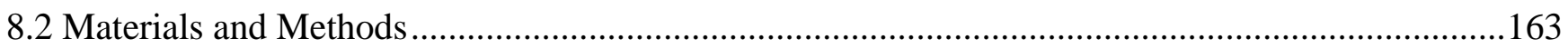

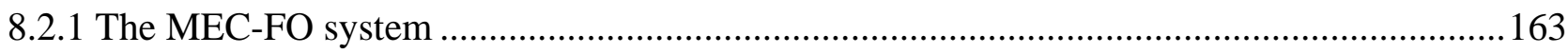

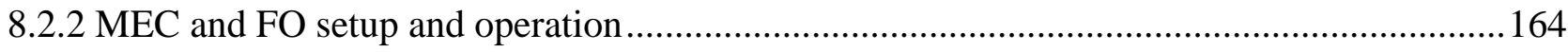

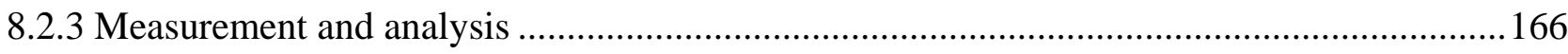

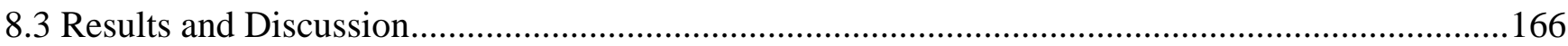

Chapter 9 Recovery of Nitrogen and Water from Landfill Leachate by A Microbial Electrolysis Cell-

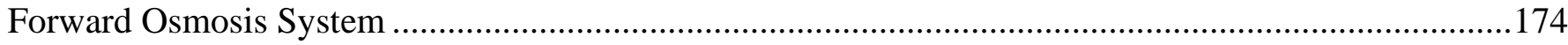

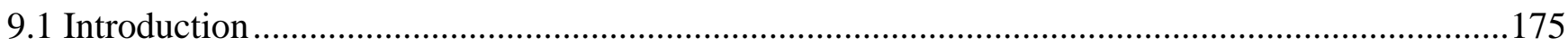

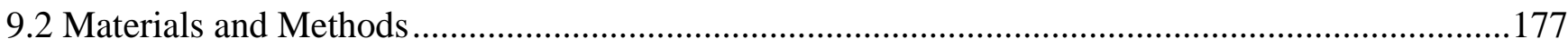

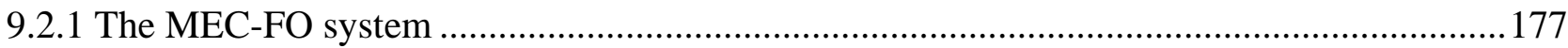

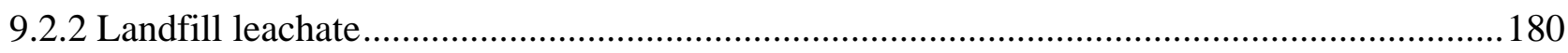

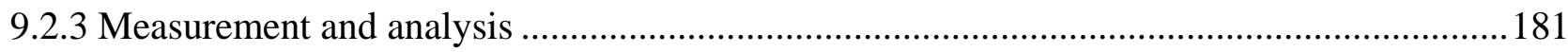

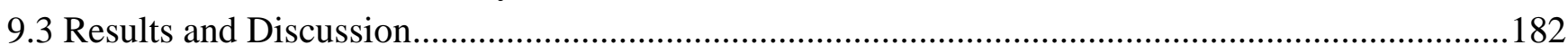

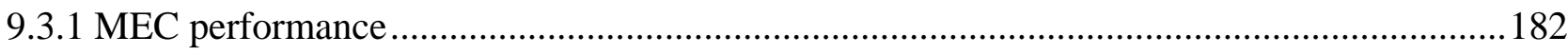

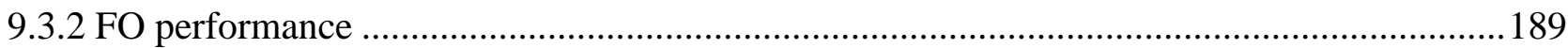

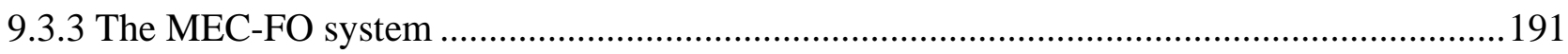

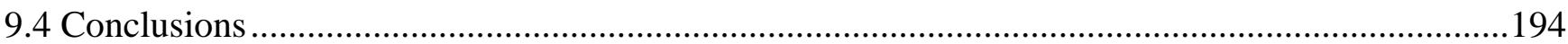

Chapter 10 Nanoparticulate $\mathrm{Ni}(\mathrm{OH})_{2}$ films synthesized from macrocyclic nickel (II) Cyclam for

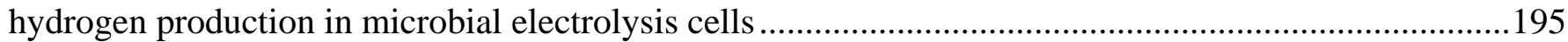

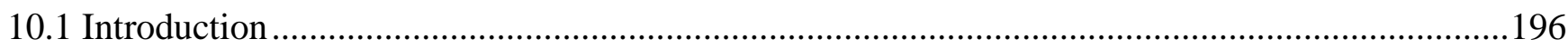

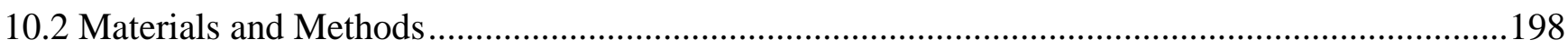

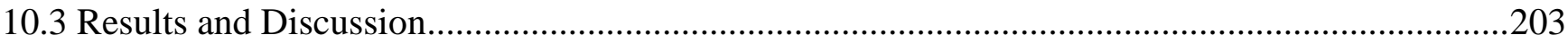

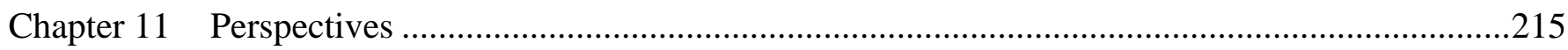

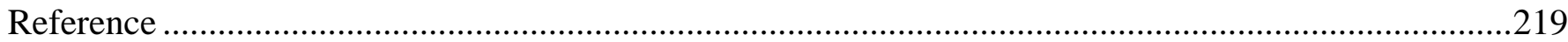




\section{Tables}

Table 2.1 Summary of the OsBES studies for energy recovery.........................................................27

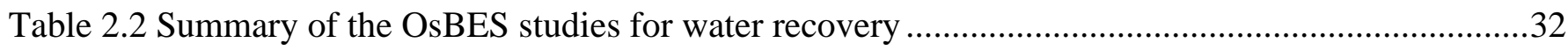

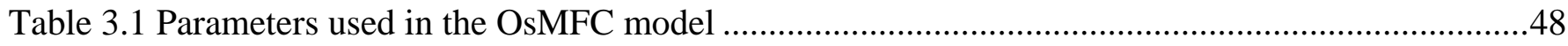

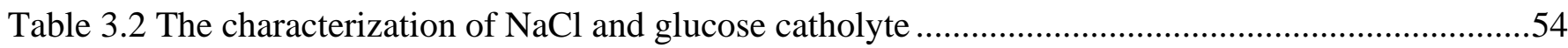

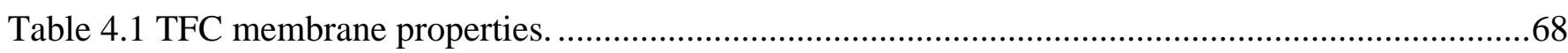

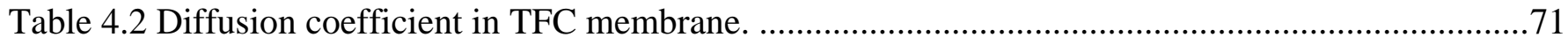

Table 6.1 Equilibrium constants for acid-base reactions and aqueous-solution equilibrium ..................111

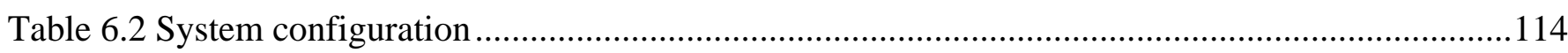

Table 6.3 Diffusion coefficients of all species ......................................................................... 115

Table 6.4 Initial conditions in the anolyte and catholyte in numerical simulations ..............................115

Table 7.1 Acid-base reactions and gas-solution equilibrium considered in our model...........................142

Table 7.2 Comparison of different technologies for ammonia removal and recovery from wastewater ..157

Table 8.1 Comparison between the MEC system, MFC-FO system and MFC ....................................172

Table 9.1 Chemical characteristics of the landfill leachate used in this study. ....................................181

Table 10.1 Hydrogen production and efficiencies of the MECs with modified carbon cloth with nickel

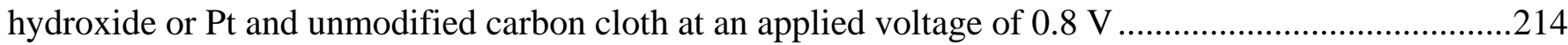




\section{Figures}

Figure 1.1 Global physical and economic surface water scarcity (Source: World Water Development Report

4. World Water Assessment Programme) (de Fraiture et al. 2007) ............................................ 1

Figure 1.2 Exmaples of BES: A) MFC; B) MEC; and C) MDC .........................................................

Figure 1.3 Microbial fuel cell architecture (a) and the different types of microorganisms in an anodic biofilm (b), including exoelectrogens that transfer electrons by direct contact (green), produce nanowires (purple) and use endogenous (and therefore self-produced) mediators (blue). other non-exoelectrogenic bacteria (brown) that live off the products produced by other bacteria or possibly use mediators or nanowires produced by other microorganisms can also be present (Logan 2009).

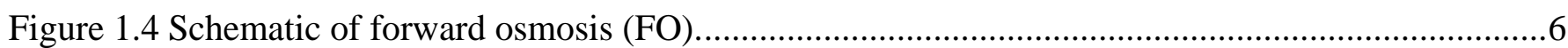

Figure 2.1 Number of published journal articles about OsBES since the first OsBES paper...................18

Figure 2.2 Schematics of (A) bioelectrochemical system (BES) and (B) forward osmosis (FO).............22

Figure 2.3 Configurations and examples of the cooperation between BES and FO: (A) integrated OsBES; (B) an example of integrated OsBES: OsMFC; (C) another example of integrated OsBES: OsMDC; (D) coupled OsBES; (E) an example of coupled OsBES: MEC-FO; and (F) another example of coupled OsBES: MFC-OMBR.

Figure 2.4 Mutual benefits between BES and FO in OsBES.

Figure 2.5 Examples of energy recovery and consumption in the OsBES: (A) an OsMFC treating synthetic municipal wastewater; (B) an MEC-FO system treating synthetic sidestream centrate.

Figure 2.6 Water recovery in the OsBES: (A) an OsMFC generates higher current than a conventional MFC at different concentrations of $\mathrm{NaCl}$ draw solution; and (B) fouled $\mathrm{FO}$ membrane greatly reduces water flux compared to pristine membrane.

Figure 3.1 The OsMFC and MFC operated in a batch mode (catholyte: $35 \mathrm{~g} \mathrm{~L}^{-1} \mathrm{NaCl}$ ): (A) open circuit voltage and the individual potentials, $(\mathrm{B})$ current density $(j)$ and the anode and cathode potential under a closed circuit condiction, and (C) water flux in open circuit (OC) and closed circuit (CC).

Figure 3.2 The OsMFC and the MFC operated with no water flux (catholyte: $2 \mathrm{~g} \mathrm{~L}^{-1} \mathrm{NaCl}$ ): (A) water flux, (B) current density, and (C) individual potentials.

Figure 3.3 The OsMFC with different catholyte conductivity: (A) current density, and (B) water flux. S1-5 are explained in Table 2

Figure 3.4 Model fitting for the OsMFC when the catholyte was changed from 35 to $2 \mathrm{~g} \mathrm{~L}^{-1} \mathrm{NaCl}$ : (A) current generation, (B) COD concentration in anode effluent, (C) salt concentrations in the

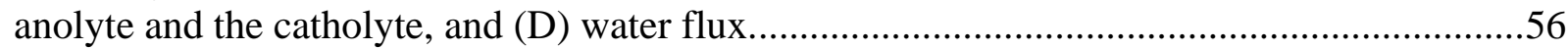

Figure 3.5 The OsMFC model validation when the catholyte was changed from $2 \mathrm{~g} \mathrm{~L}^{-1} \mathrm{NaCl}$ to $10 \mathrm{~g} \mathrm{~L}^{-1}$ $\mathrm{NaCl}$ solution: (A) current generation, (B) salt concentrations of anolyte and catholyte, and (C) water flux.

Figure 3.6 Model prediction for the OsMFC and the MFC with varied salt concentration in the catholyte: (A) current generation in OsMFC and MFC, (B) internal resistance distribution in MFC, and (C) internal resistance distribution in OsMFC. $\mathrm{R}_{\mathrm{m}}$ : membrane resistance; RC: catholyte

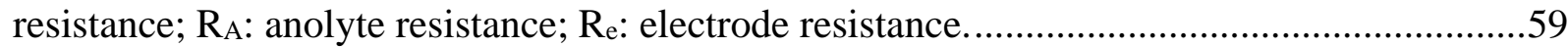

Figure 4.1 Schematic of an osmotic microbial fuel cell.

Figure 4.2 Photograph of OsMFC. A, anolyte reservoir; B, catholyte reservoir; C, OsMFC reactor; D, pump for $\mathrm{pH}$ adjustment; $\mathrm{E}, 1 \mathrm{M} \mathrm{NaOH} / \mathrm{HCl}$ solution; F, recirculation pump; $\mathrm{G}$, stir plate; $\mathrm{H}$, mass balance; I, pH meter; J, pump speed controller, and $\mathrm{K}$, aeration pump (for catholyte). 
Figure 4.3 Integrity test for the FO membrane. (A) the extracted water volume during the 2-h operation for the pristine FO membrane and membrane after-wash; (B) the water flux and RSF for the pristine

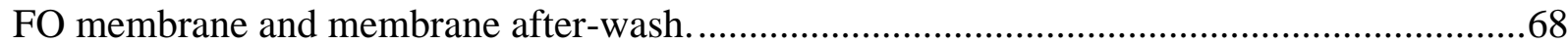

Figure 4.4 The OsMFC performance with different external resistance: (A) current generation; and (B)

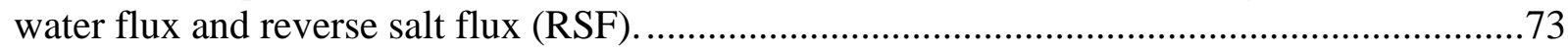

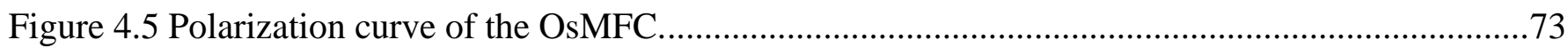

Figure 4.6 Water flux in OsMFC with different external resistance (Coulomb production): (A) $1 \Omega-311$ Coulomb (C) generation (B) $10 \Omega-226$ C generation (C) $40 \Omega-148$ C generation (D) $100 \Omega$ -

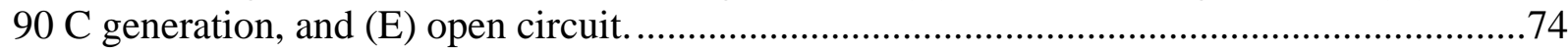

Figure $4.7 \mathrm{pH}$ variation in anolyte and catholyte with fixed catholyte $\mathrm{pH}$. The external resistance was 40

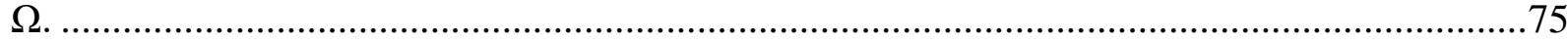

Figure 4.8 The effects of the controlled catholyte $\mathrm{pH}$ and external resistance: (A) RSF; and (B) total Coulomb production. Quasi-infinite resistance was in open circuit mode and didn't have current

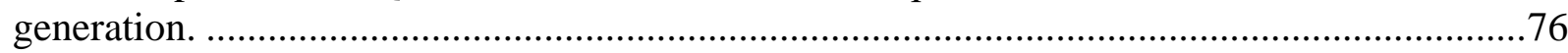

Figure 4.9 Performance of the OsMFC with $40 \Omega$ resistor and uncontrolled catholyte $\mathrm{pH}$ : (A) water flux

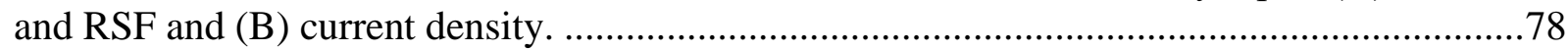

Figure 4.10 The OsMFC performance with uncontrolled catholyte $\mathrm{pH}$ : (A) measured (actual) and calculated $\mathrm{pH}$ value with different initial catholyte $\mathrm{pH}$ (external resistance of $40 \Omega$ ); and (B) water flux and RSF with different external resistance (initial catholyte $\mathrm{pH}$ of 7) ........................................78

Figure 4.11 Water flux and RSF of the OsMFC with open circuit and uncontrolled catholyte pH. ...........80

Figure 4.12 Transport of sodium $\left(\mathrm{Na}^{+}\right)$and chloride $\left(\mathrm{Cl}^{-}\right)$ions to the anode: (A) affected by Coulomb production; and (B) affected by the catholyte $\mathrm{pH}$.

Figure 4.13 Proposed mechanism of the ion transport (orange: $\mathrm{Na}^{+}$; green: $\mathrm{Cl}^{-}$) across the TFC membrane The arrows represent the flux of ions (orange: reverse flux of $\mathrm{Na}^{+}$; green: reverse flux of $\mathrm{Cl}^{-}$ dragged by $\mathrm{Na}^{+}$transport; yellow: forward flux of $\mathrm{Na}^{+}$driven by current; purple: reverse $\mathrm{Na}^{+}$ flux inhibited by current; red: increased reverse flux of $\mathrm{Na}^{+}$by increased catholyte $\left.\mathrm{pH}\right) . \ldots \ldots . .82$

Figure 4.14 Contribution of diffusion and EDM (electrically-driven migration) to ion transport: (A) affected

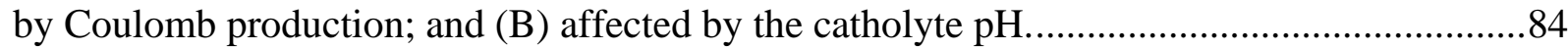

Figure 5.1 Ammonium removal by the OsMFC affected by different catholyte $\mathrm{NaCl}$ concentrations: (A) current generation; and (B) water flux and $\mathrm{NH}_{4}{ }^{+}-\mathrm{N}$ removal efficiency.

Figure 5.2 Ammonium removal by the OsMFC affected by different anolyte flow rates: (A) current generation and $\mathrm{NH}_{4}{ }^{+}-\mathrm{N}$ removal efficiency; and (B) water flux.

Figure 5.3 Ammonium removal by the OsMFC affected by the current generation: (A) current generation in open and closed circuit conditions; (B) $\mathrm{NH}_{4}{ }^{+}-\mathrm{N}$ removal efficiency in closed and open circuit conditions; (C) polarization curve; and (D) current generation and $\mathrm{NH}_{4}{ }^{+}-\mathrm{N}$ removal efficiency with different external resistances.

Figure 5.4 Ammonium removal by the OsMFC affected by water flux: (A) similar current generation under two different conditions, $2 \mathrm{~g} \mathrm{~L}^{-1} \mathrm{NaCl}$ as catholyte $/ 10 \Omega$ external resistance and $35 \mathrm{~g} \mathrm{~L}^{-1} \mathrm{NaCl}$ as catholyte / $88 \Omega$ external resistance; and (B) $\mathrm{NH}_{4}{ }^{+}-\mathrm{N}$ removal efficiency and water flux under those two conditions.

Figure 5.5 The contribution of different mechanisms to ammonium removal: (A) comparison between open and closed circuit conditions with or without water flux; (B) effects of current generation; and (C) effects of water flux (orange: current generation; green: water flux; yellow: ion exchange; and maroon: diffusion). 101

Figure 6.1 A sketch of the MEC-based ammonia recovery system. 110

Figure 6.2 Comparison of experimental and model studies of ammonia recovery during three batch cycles of MEC operation. The current density measured experimentally (a) is feed into the models to predict the various observables of the anolyte and the catholyte: the $\mathrm{pH}(\mathrm{b})$, the acetate 
concentration (c), the amount of $\mathrm{NH}_{3}$ collected from catholyte by aeration (d), the total nitrogen content (e), and the transport of $\mathrm{Na}^{+}$and $\mathrm{Cl}^{-}$ions out of the anolyte (f). Symbols are the experiment data and lines are model prediction. In (b-e), red lines are for anolyte and blue lines are for catholyte. In (c), the acetate concentration in catholyte is below the detection limit of our equipment and thus not shown. In (f), to highlight the relative importance of $\mathrm{Na}^{+} / \mathrm{Cl}^{-}$ion transport in the overall charge transport in the system, their transport is quantified using the cumulative transport number $\boldsymbol{t} \boldsymbol{i}+$ (see text, $\boldsymbol{t} \boldsymbol{i}+=\mathbf{1 . 0}$ means that the charge carried by the transport a species $i$ is equal to the total charge passed through the membrane). A detailed summary of operating conditions and parameters (e.g., initial species concentration in anolyte/catholyte) is in provided in Table 6.2, Table 6.3, and Table 6.4.

Figure 6.3 Partition between $\mathrm{NH}_{4}{ }^{+}$and $\mathrm{NH}_{3}$ as a function of $\mathrm{pH}$ assuming fast $\mathrm{NH}_{3}+\mathrm{H}^{+}=\mathrm{NH}_{4}{ }^{+}$reaction.

Figure 6.4 Regulation of $\mathrm{pH}$ in $\mathrm{MEC}$ during ammonia recovery operation. The variation of the $\mathrm{pH}$ values in the anolyte (a) and the catholyte (c) is governed by various Faradaic and chemical reactions (b and d) identified in Equation 6.11-6.15. The generation/consumption of proton and hydroxide by the chemical reactions involving various ion groups in the anolyte and catholyte are shown in (b) and (d), respectively. These generation/consumption terms are normalized by the production of proton (in anolyte, for panel b) and hydroxide (in catholyte, for panel d) generated through Faradic reactions. [Operating conditions and parameters in this study are the same as in Figure 6.2 except the followings: the current is $\mathrm{I}=1 \mathrm{~A} / \mathrm{m}^{2}$; the catholyte initially contains $10 \mathrm{mM}$ of $\mathrm{Na}^{+}$ions and the accompanying carbonate group ions to produce a $\mathrm{pH}$ of $\left.\sim 6.8\right]$.

Figure 6.5 Mechanisms of $\mathrm{NH}_{4}{ }^{+} / \mathrm{NH}_{3}$ transport in MECs. (a) Relative strength of $\mathrm{NH}_{4}{ }^{+}$ion transport due to diffusion and migration. (b) Evolution of the $\mathrm{NH}_{4}{ }^{+}$ion concentration in the anolyte and catholyte. (c) Distribution of $\mathrm{pH}$ across the CEM and at the CEM-anolyte/catholyte interfaces. (d) The diffusion flux of $\mathrm{NH}_{3}$ toward the anolyte at the CEM-catholyte/anolyte interfaces. These diffusion fluxes are normalized by the total $\mathrm{NH}_{4}{ }^{+}$ion flux to highlight their impact on the ammonia recovery in the catholyte. [ALL parameters in this study are the same as in Fig. 3.]

Figure 6.6 Ionic competition between $\mathrm{Na}^{+}$and $\mathrm{NH}_{4}^{+}$. (a) Transport number of $\mathrm{Na}^{+}$and $\mathrm{NH}_{4}{ }^{+}$ions at the CEM-anolyte interface. (b) The concentration profiles and transport of $\mathrm{Na}^{+}$and $\mathrm{NH}_{4}{ }^{+}$ions within the cation exchange membrane. [ALL parameters in this study are the same as in Chapter 5.]

Figure 6.7 Transport number of $\mathrm{Na}^{+}$and $\mathrm{NH}_{4}{ }^{+}$ions at the CEM-anolyte interface under different initial $\mathrm{Na}^{+}$ ion concentration in the anolyte. The solid (dashed) lines are for case in which the initial concentration of $\mathrm{Na}^{+}$ions in the anolyte is equal to (three times of) that of the $\mathrm{NH}_{4}{ }^{+}$ions....130

Figure 6.8 Evolution of the $\mathrm{NH}_{4}{ }^{+}$ion concentration in anolyte during operations with different initial $\mathrm{Na}^{+}$ ion concentrations in the anolyte. S0.2, S1, and S3 corresponds to the case in which the initial $\mathrm{Na}^{+}$ion concentration in the anolyte is $0.2,1.0$ and 3.0 (for demonstration purpose) times of the initial $\mathrm{NH}_{4}{ }^{+}$ion concentration, respectively. The operating parameters (e.g., current density and aeration rate) are otherwise identical in all cases.

Figure 7.1 Ammonia recovery by the BES affected by different catholyte aeration rates: (A) current generation; (B) $\mathrm{NH}_{4}{ }^{+}-\mathrm{N}$ concentration in the anolyte and the catholyte; (C) the $\mathrm{NH}_{3}$ recovery rate; and (D) simulated anolyte and catholyte $\mathrm{NH}_{4}{ }^{+}-\mathrm{N}$ concentration. The external resistance was $1 \Omega$ and the external voltage was $0.8 \mathrm{~V}$.

Figure 7.2 The total Coulomb generation per cycle for BES with different aeration rate. 145

Figure 7.3 The simulated amount of $\mathrm{NH}_{3}$ collected from catholyte by aeration with different catholyte aeration rate 
Figure 7.4 Energy consumption by the BES affected by different aeration rate: (A) energy consumption for ammonium removal; and (B) energy consumption for ammonia recovery (blue: aeration; orange: power supply; yellow: catholyte recirculation; green: anolyte recirculation). 148

Figure 7.5 Ammonia recovery by the BES affected by different external voltages: (A) current generation; (B) $\mathrm{NH}_{4}{ }^{+}-\mathrm{N}$ concentration in the anolyte and the catholyte effluent; (C) the $\mathrm{NH}_{3}$ recovery rate; and (D) the simulated anolyte and catholyte $\mathrm{NH}_{4}{ }^{+}-\mathrm{N}$ concentrations. The external resistance was $1 \Omega$ and the catholyte aeration rate was $100 \mathrm{~mL} \mathrm{~min}^{-1}$

Figure 7.6 The total Coulomb generation per cycle for BES with different external voltage..................150

Figure 7.7 The simulated amount of ammonia recovery for BES with different current........................150

Figure 7.8 Energy consumption for ammonia recovery affected by different external voltages (Green: anolyte recirculation; yellow: catholyte recirculation; orange: power supply; and cyan: aeration).

Figure 7.9 The BES performance affected by different external resistances: (A) current generation; (B) the $\mathrm{NH}_{3}$ recovery rate; and $(\mathrm{C})$ simulated $\mathrm{NH}_{4}{ }^{+}-\mathrm{N}$ concentration in the anolyte and catholyte effluents. The external voltage was $0.8 \mathrm{~V}$ and the catholyte aeration rate was $100 \mathrm{~mL} \mathrm{~min}^{-1} 153$

Figure 7.10 The total Coulomb generation per cycle for BES with different external resistance.............154

Figure 7.11 The $\mathrm{NH}_{4}{ }^{+}-\mathrm{N}$ concentration for anolyte and catholyte effluent in BES with different external resistances.

Figure 7.12 Energy consumption for ammonia recovery affected by different external resistances (Gray: anolyte recirculation; yellow: catholyte recirculation; cyan: power supply; and magenta: aeration). 155

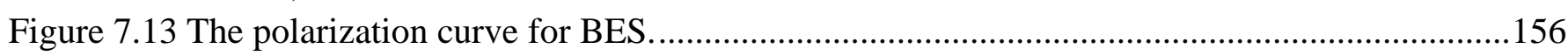

Figure 8.1 The schematic design of the MEC-FO coupled system ................................................... 164

Figure 8.2 Ammonia recovery in the MEC: (A) current density, (B) COD concentration, (C) ammonium nitrogen concentration in the MEC and the solution of absorption bottle, and (D) the $\mathrm{pH}$ in each chamber.

Figure 8.3 The MEC-FO system: (A) water flux in the FO process, (B) reverse salt flux in FO, the (C) current density from the mixture of returned feed solution and fresh anolyte in the MEC, and (D) ammonium nitrogen concentration in the MEC and the FO ........................................169

Figure 9.1 Schematic of the MEC-FO system.......................................................................178

Figure 9.2 The MEC performance fed with and without aeration: (A) current generation; (B) average effluent COD and Coulumbic recovery (CR); and (C) the MEC effluent conductivity..........184

Figure 9.3 Ammonium recovery in the MEC: (A) ammonium concentration with aeration; (B) (A) ammonium concentration without aeration; and $(\mathrm{C})$ the current generation when aeration was put back to the MEC. The insets in (A) and (B) show the $\mathrm{NH}_{4}{ }^{+}-\mathrm{N}$ removal efficiency..........186

Figure 9.4 The concentrations of the major ions in the MEC with aeration and without aeration (A) in the anolyte from the $3^{\text {rd }}$ batch; (B) in the catholyte from the $3^{\text {rd }}$ batch; (C) in the anolyte from the $5^{\text {th }}$ batch; and (D) in the catholyte from the $5^{\text {th }}$ batch. 188

Figure 9.5 The FO performance fed with the treated leachate: (A) water flux with $1 \mathrm{M} \mathrm{NH}_{4} \mathrm{HCO}_{3}$ as draw solution; (B) reverse salt flux with different $\mathrm{NH}_{4} \mathrm{HCO}_{3}$ concentrations; and (C) EIS for new and used FO membranes. The inset in (C) shows the water flux with $0.5 \mathrm{M} \mathrm{NH}_{4} \mathrm{HCO}_{3}$ as draw solution for new and used FO membranes.

Figure 9.6 The MEC-FO system performance: (A) current generation; and (B) $\mathrm{NH}_{4}{ }^{+}-\mathrm{N}$ distribution during

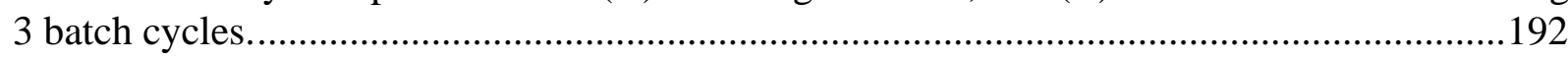

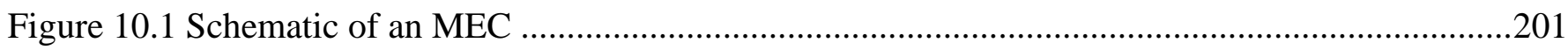

Figure 10.2 (a) Cyclic voltammograms of $\mathrm{Ni}$ (II)cyclam (solid black line) and $\mathrm{Ni}(\mathrm{II}) \mathrm{Cl}_{2} \bullet 6 \mathrm{H}_{2} \mathrm{O}$ in an aqueous $0.1 \mathrm{M} \mathrm{NaOH}$ solution. The inset shows a closer view of the $\mathrm{Ni}(\mathrm{II}) \mathrm{Cl}_{2} \cdot 6 \mathrm{H}_{2} \mathrm{O} \mathrm{CV}$. (b) Comparison of the current densities corresponding to the electrodeposition of the $\mathrm{Ni}$ (II) catalyst from the 
$\mathrm{Ni}(\mathrm{II})$ cyclam (black) and $\mathrm{Ni}(\mathrm{II}) \mathrm{Cl}_{2}$ solutions on the $\mathrm{CFE}$ as a function of the number of potential sweep scans. 204

Figure 10.3 (a) Cyclic voltammograms of $6 \mathrm{mM}$ (solid black line), $15 \mathrm{mM}$ (solid red line), and $23 \mathrm{mM}$ (solid blue line) $\mathrm{Ni}$ (II)cyclam solutions in aqueous $0.1 \mathrm{M} \mathrm{NaOH}$ electrolyte. (b) Comparison of the current densities corresponding to the electrodeposition of the $\mathrm{Ni}$ (II) catalyst from the 6 $\mathrm{mmm}$ (black circles), $15 \mathrm{mM}$ (red circles), and $23 \mathrm{mM}$ (blue circles) Ni(II)cyclam solutions on the CFE as a function of the number of potential sweep scans. These are compared to each other by dividing the current densities by the concentration of $\mathrm{Ni}$ (II) cyclam at the start of the deposition. 205

Figure 10.4 Cyclic voltammograms of a $\mathrm{Ni}(\mathrm{OH})_{2}$ film deposited on $\mathrm{CFE}$ (black - left y-axis), carbon cloth (black dash - left y-axis), and Ni(II)cyclam in solution (red - right y-axis, working electrode: glassy carbon) in anaerobic $0.1 \mathrm{M} \mathrm{TBAPF}_{6}-\mathrm{CH}_{3} \mathrm{CN}$ solutions (scan rate $25 \mathrm{mV} / \mathrm{s}$ ). 206

Figure 10.5 X-ray photoelectron spectra of $\mathrm{Ni} 2 \mathrm{p}_{3 / 2}$ (top), $\mathrm{N}$ 1s (middle), and $\mathrm{O} 1 \mathrm{~s}$ (bottom) signals for films prepared from $6 \mathrm{mM}, 15 \mathrm{mM}$, and $23 \mathrm{mM}$ solutions of $\mathrm{Ni}(\mathrm{II})$ cyclam. 207

Figure 10.6 SEM images of carbon cloth ( $a$ and $b$ ) and $\mathrm{Ni}(\mathrm{OH}) 2$-modified carbon cloth that results from electrodeposition from a Ni(II)cyclam precursor solution at concentration of $23 \mathrm{mM}$ (c and d), $15 \mathrm{mM}(\mathrm{e})$ and $6 \mathrm{mM}(\mathrm{f})$.

Figure 10.7 a. Tafel slope for carbon cloth (green), $\mathrm{Ni}(\mathrm{OH})_{2}-6 \mathrm{mM}$ precursor (navy), $\mathrm{Ni}(\mathrm{OH})_{2}-15 \mathrm{mM}$ precursor (red) and $\mathrm{Ni}(\mathrm{OH})_{2}-23 \mathrm{mM}$ precursor (black) with a scan rate of $1 \mathrm{mV} \mathrm{s}^{-1}$ measured in PBS buffer (1 M). b-d. CV in the region of 0.1-0.2 V vs. RHE plotted against scan rate and linear regression for the $C_{d l}$ estimation with $23 \mathrm{mM}, 15 \mathrm{mM}$ and $6 \mathrm{mM} \mathrm{Ni}(\mathrm{II})$ cyclam. Scan rate: $130 \mathrm{mV} / \mathrm{s}$ (orange), $100 \mathrm{mV} / \mathrm{s}$ (blue), $80 \mathrm{mV} / \mathrm{s}$ (navy), $50 \mathrm{mV} / \mathrm{s}$ (green), $30 \mathrm{mV} / \mathrm{s}$ (black), and $10 \mathrm{mV} / \mathrm{s}$ (red). 210

Figure 10.8 EDS spectrums of (a) unmodified carbon cloth, (b) $\mathrm{Ni}(\mathrm{OH}) 2$ modified carbon cloth. Pd and $\mathrm{Au}$ peaks results from the metal pre-treatment of the samples prior to SEM collection 212

Figure 10.9 Current density of the MEC with modified carbon cloth with $\mathrm{Ni}(\mathrm{OH}) 2(23 \mathrm{mM} \mathrm{Ni}(\mathrm{II})$ cyclam (black), $15 \mathrm{mM}$ (green), and $6 \mathrm{mM}$ (pink)), $\mathrm{Ni}(\mathrm{OH}) 2$ from $\mathrm{Ni}(\mathrm{II}) \mathrm{Cl} 2 \cdot 6 \mathrm{H} 2 \mathrm{O}$ (orange) or $\mathrm{Pt}$ (red) and unmodified carbon cloth (blue) at an applied voltage of $0.8 \mathrm{~V}$. 


\section{Chapter 1 Introduction}

\subsection{Water Scarcity and Wastewater Treatment}

Water resource scarcity, variability, and uncertainty are becoming more and more prominent all around the world. According to a recent report (Water 2014), 768 million people lack access to fresh water, and another 2.5 billion suffer from sanitation problems. $25 \%$ of world population, $33 \%$ of developing world population will live in areas of water scarcity in 2025 (Figure 1.1) (de Fraiture et al. 2007). In addition to the deterioration in ecosystems and hydrological cycle, global water consumption is estimated to increase by $55 \%$ by year 2050 , resulting in significantly increased freshwater demand (Water 2014). The growing water stress promotes the development

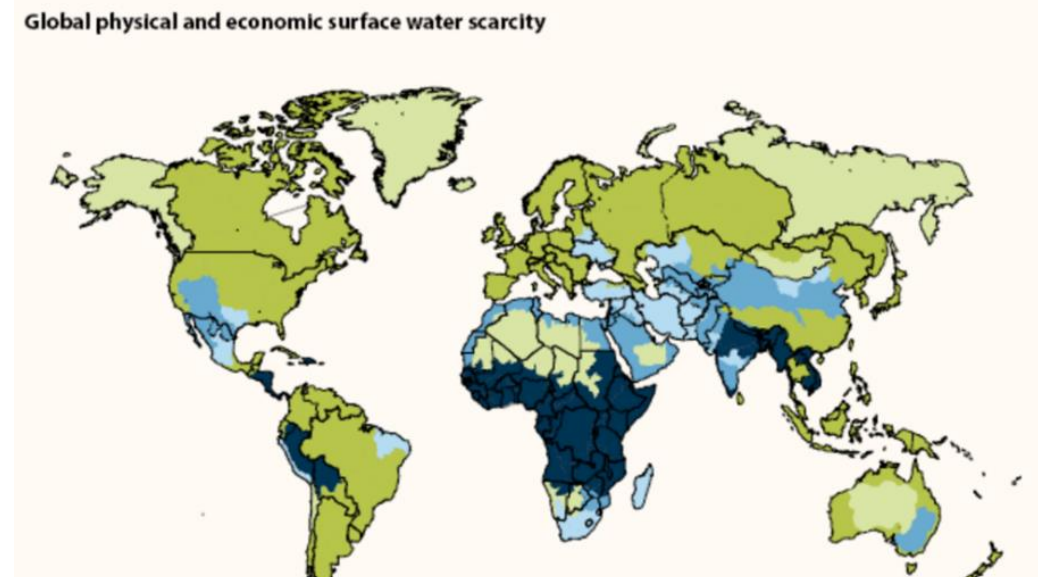

Figure 1.1 Global physical and economic surface water scarcity (Source: World Water Development Report 4. World Water Assessment Programme) (de Fraiture et al. 2007).

of wastewater reclamation as a new water resource.

Besides water, wastewaters also contain many other valuable sources, such as nutrient and energy, which are critically important and have a wide range of applications. Globally, approximately $20 \%$ of manufactured nitrogen and phosphorous is contained in domestic wastewater (Batstone et al. 
2015, Matassa et al. 2015a), of which the majority is potentially recoverable due to urban concentration. The situation is more attenuated for energy. Wastewater contains $1.3 \mathrm{MJ} / \mathrm{person} / \mathrm{day}$ of chemical energy (Batstone et al. 2015). This represents 1\% of the current world total energy consumption, or $4 \%$ of the world total electricity production, and requires a process to convert it from dilute chemical energy to a usable form (Puyol et al. 2016).

Nutrient, Energy, and Water (NEW) recovery from wastewater has been accomplished in separate processes such as anaerobic digestion (energy), struvite recovery (nutrient) and membrane treatment (water), but each of them has application challenges. For example, anaerobic digestion is usually not applied to low-strength wastewater or small-volume wastewater, struvite recovery is essentially a phosphorus recovery process and ammonia is not efficiently recovered, and pressure-driven membrane treatment is an energy-intensive process. Therefore, there is a strong need for innovative treatment technologies that can accomplish NEW recovery in a resource- and energy-efficient way.

\subsection{Introduction to Bioelectrochemical system}

Bioelectrochemical system (BES) is a group of technologies that relies on the interaction between microorganisms and electrode (Rabaey et al. 2009). In a BES anode, organic compounds in wastewater are oxidized by the exoelectrogens growing on an anode electrode and the generated electrons spontaneously flow from the anode electrode to the cathode electrode, where oxygen is reduced to water (oxygen reduction reaction) (Rabaey and Verstraete 2005). Ion exchange membranes such as cation exchange membrane (CEM) or anion exchange membrane (AEM) is used as the separator between the anode and the cathode, and there have also been membrane-less 
BES reactors that still need a separator between the electrodes (Li et al. 2011b). A representative BES is a microbial fuel cell (MFC), which can generate electricity directly from wastewater (Li et al. 2014b). MFCs can be modified to accomplish various functions such as hydrogen production, desalination, and heavy metal removal (Figure 1.2A) (Logan and Rabaey 2012, Wang and Ren 2013). In a microbial electrolysis cell (MEC), hydrogen gas or other value-added chemicals can be produced with a small amount of external power input (Figure 1.2B) (Cheng et al. 2009, Logan et al. 2008a). A microbial desalination cell (MDC) can achieve desalination of saline water in a similar way to that of electrodialysis but with much lower energy demand (Figure 1.2C) (Cao et al. 2009). To produce higher-value products such as pure organic compounds, electrons are used by microorganisms in the cathode to achieve microbial electrosysthesis (Rabaey and Rozendal 2010).

BES could accomplish direct biological conversion of organic energy into electricity. The process of transferring electrons to electrode is achieved in anaerobic bacteria respiration by a wide microbial diversity. The most frequently used model microorganisms
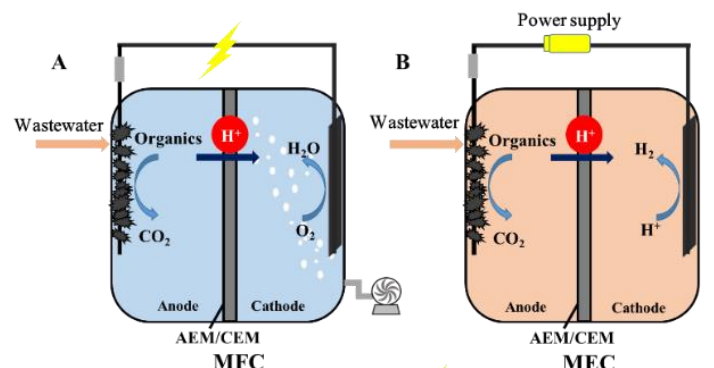

C

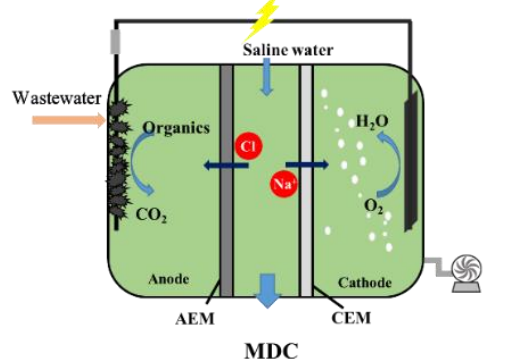

Figure 1.2 Exmaples of BES: A) MFC; B) MEC; and C) MDC. 


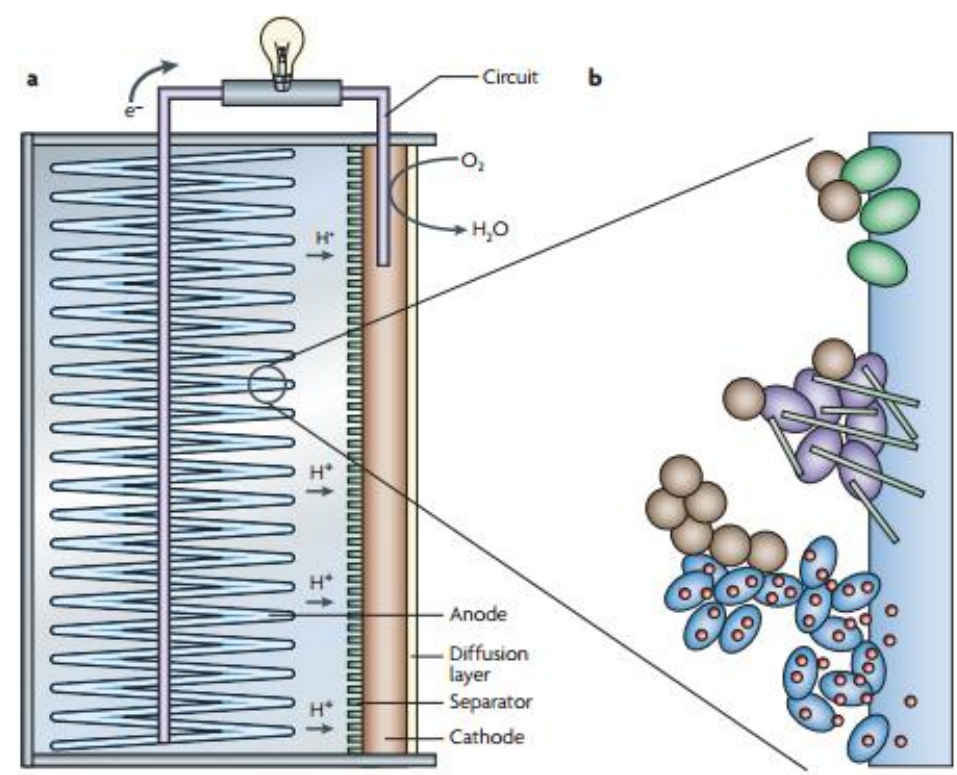

Figure 1.3 Microbial fuel cell architecture (a) and the different types of microorganisms in an anodic biofilm (b), including exoelectrogens that transfer electrons by direct contact (green), produce nanowires (purple) and use endogenous (and therefore self-produced) mediators (blue). other non-exoelectrogenic bacteria (brown) that live off the products produced by other bacteria or possibly use mediators or nanowires produced by other microorganisms can also be present (Logan 2009).

are Shewanella and Geobacter genera (Yang et al. 2012). The microorganisms will, with their capacity, attempt to maximize their energy gain by selecting the electron acceptor with the highest potential available. Several mechanisms have been described for extracellular electron transfer (EET) in the past years, they can be categorized as direct electron transfer and indirect electron transfer (Figure 1.3) (Rabaey et al. 2009). Direct electron transfer involves membrane bound or associated enzyme complexes, and may involve conductive pili or pilus-like structures (also called nanowires). Indirect electron transfer uses organic or inorganic shuttles. The soluble compound is reduced or oxidized at the cell and subsequently diffuses towards the insoluble electron acceptor/donor.

Wastewater could be used as a source of fuel for BES, with the benefit of accomplishing wastewater treatment (Liu et al. 2004). Recent years, BES has been researched on treating 
wastewater and extracting the waste energy extensively, with the representing technology, microbial fuel cell (MFC). For example, MFCs may produce up to $1.43 \mathrm{kWh} \mathrm{m}^{-3}$ from a primary sludge or $1.8 \mathrm{kWh} \mathrm{m}^{-3}$ from a treated effluent (Ge et al., 2013). Theoretically, BES can convert maximum $100 \%$ of chemical energy into electricity. However, there is always some energy lost through 1) coulombic loss where organics are not converted to electrical current at $100 \%$, and 2) electrochemical potential or voltage loss. Nevertheless, the reported energy conversion efficiency for MFC can reach $80 \%$ which is much higher than $33 \%$ for typical heat engine combustion of methane gas (Rabaey et al. 2005).

In BES, nitrogen could be removed through bioelectrochemical denitrification or recovered via ammonium migration driven by electricity generation (Clauwaert et al. 2007, Kuntke et al. 2012). Nitrogen recovery in BES is considered as an efficient and economical method for nitrogen removal from wastewater (Kelly and He 2014). When cation exchange membranes (CEMs) are used (Cheng et al. 2013, Haddadi et al. 2013, Logan et al. 2008b, Rozendal et al. 2006a), the current is carried by cations such as $\mathrm{NH}_{4}{ }^{+}$ions moving from the anolyte into the catholyte through the CEM. The $\mathrm{NH}_{4}{ }^{+}$ions, once transported into the catholyte, are converted into $\mathrm{NH}_{3}$ molecules because the catholyte is usually rendered basic by the production of $\mathrm{OH}^{-}$ions therein (Haddadi et al. 2014, Kim et al. 2008, Kuntke et al. 2012). Some of these $\mathrm{NH}_{3}$ molecules can then be recovered through gas aeration. This type of process has been demonstrated in various BES including microbial fuel cells (MFCs) and microbial electrolysis cells (MECs). In MECs, a higher current density would greatly enhance ammonia recovery (Haddadi et al. 2013), and thus MECs with external power exhibit a better performance for ammonium recovery than MFCs (Zhang et al. 2014a). It was reported that ammonia can be recovered from ammonium-rich wastewater, such as 
synthetic wastewater, urine, and swine wastewater (Kim et al. 2008, Kuntke et al. 2011, Kuntke et al. 2012, Qin and He 2014).

\subsection{Introduction to Forward Osmosis}

Forward osmosis (FO) is based on the natural phenomenon of osmotic processes, and can extract clean water from wastewater (Cath et al. 2006). In an FO process (Figure 1.4), water moves across a semipermeable membrane from high water potential to low water potential, driven by an osmotic pressure gradient (Zhang et al. 2011a). Draw solutes such as $\mathrm{NH}_{3} / \mathrm{CO}_{2}$ and magnetic nanoparticles can avoid energy-intensive post-treatment processes like reverse osmosis (Ling et al. 2010, McCutcheon et al. 2005). Fertilizers can also be applied as draw solutes to eliminate the need for regeneration (Phuntsho et al. 2011). Because of small pore radius $(0.25-0.37 \mathrm{~nm})$, FO can achieve effective rejection of a wide range of contaminants (Coday et al. 2014). The lack of external pressure input also results in cost-saving and energy-efficient water extraction and low membrane fouling (Lutchmiah et al. 2014b). Thus, FO is considered as a promising technology for extracting reusable water from complex wastewater such as landfill leachate (Cath et al. 2005a, Cath et al. 2005b, Cornelissen et al. 2011, Holloway et al. 2007, Zhang et al. 2014d), and dewatering of waste activated sludge (Chung et al. 2012a, Cornelissen et al. 2008, Zhu et al. 2012).

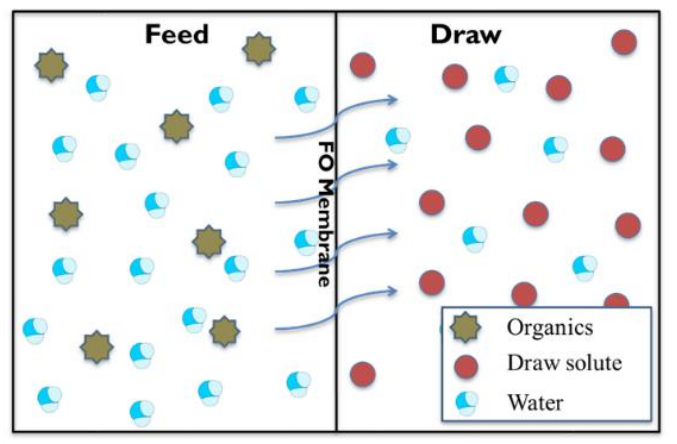

Figure 1.4 Schematic of forward osmosis (FO). 
FO can also be applied to produce electric energy in a process called pressure retarded osmosis (PRO), which takes advantage of increased hydraulic pressure due to water expansion of draw solution and uses pressurized flow of water to drive a hydro turbine for power production (Achilli and Childress 2010). The details of FO membrane, limitation, development and potential applications can be found in various review papers (Cath et al. 2006, Chung et al. 2012b, Lutchmiah et al. 2014b, Thompson and Nicoll 2011, Zhao et al. 2012). In FO, reversed salt flux (RSF) is one of the most challenging drawbacks of FO (Chekli et al. 2012). RSF occurs due to a concentration gradient across an FO membrane, resulting in backward migration of draw solutes into the feed side. Such reverse migration reduces the driving force for water transport, increases the operation cost due to loss of draw solutes, and contaminates the feed solution (Cath et al. 2006). RSF can also result in salt accumulation within the fouling layer on the membrane surface, which increases the cake-enhanced osmotic pressure and therefore, exhibits a negative impact on the

process efficiency (Boo et al. 2012). The reverse salt flux was influenced by various parameters: draw solute, solution pH, etc. (Phillip et al. 2010, Yong et al. 2012). Recent studies demonstrated that the ion transport across FO membrane varied significantly with changes in $\mathrm{pH}$ and occurred fastest at alkaline $\mathrm{pH}$ (Arena et al. 2015).

\subsection{Osmotic Bioelectrochemical Systems}

The individual advantages of BES (e.g., energy recovery and nutrient recovery) and FO (water recovery) intrigue their cooperation to accomplish NEW recovery, and such cooperation would also address the disadvantages of each technology (Lu et al. 2014b). Osmotic bioelectrochemical system (OsBES), an innovative treatment concept, is proposed. An OsBES can accomplish simultaneous treatment of wastewater and recovery of resources such as nutrient, energy, and water. 
The synergy between those two technologies lies in the complementing functions in energy recovery and water/wastewater treatment. With the advantage of effective water extraction, an FO process can improve water recovery from the treatment by BES. Pressure retard osmosis (PRO) process may provide BES with sufficient and sustainable energy via water flux. In return, BES can achieve degradation of contaminants in wastewater, provide source of treated wastewater for recovery, and harvest valuable products to improve the sustainability of the whole system. A proper combination of the two may also solve some key issues in each process, such as the supply of draw solute, reverse solute leakage in the FO, and energy requirement for carrying out certain reactions in the BES. The first study of BES-FO cooperation appeared in 2011 and proposed a new concept of osmotic microbial fuel cells (OsMFCs) (Zhang et al. 2011a). Since then, there have been various studies and efforts to explore the cooperation between BES and FO from different aspects and this will be explicitly explained in Chapter 217.

The cooperation between BES and FO can be accomplished in two ways depending on the location of FO membrane, integrated OsBES (internal cooperation) and coupled OsBES (external cooperation). Integrated OsBES uses FO membrane to replace ion exchange membrane and installs FO membrane within the BES as a separator between an anode and a cathode. The first OsBES was created as an integrated system, in the form of OsMFCs (Zhang et al. 2011a). The anolyte (wastewater) in an OsMFC also acts as the feed solution for the FO process, while a highconcentration catholyte is used as the draw solution. The feasibility of this OsMFC was demonstrated by successful water extraction and bioelectricity generation. The concept of OsMFC was further developed to create osmotic microbial electrolysis cells (OsMECs) and microbial desalination cells (OsMDCs) (Lee et al. 2015, Zhang and He 2012). In an OsMEC, external power supply is applied to drive hydrogen evolution reaction in the cathode. In an OsMDC, AEM that 
separates the anode and the middle compartment is replaced by FO membrane, while CEM is kept between the cathode and the middle compartment. In this way, saline water in the middle compartment would function as a draw solution to extract high quality water from the anolyte, and salts can still be removed driven by electricity generation like that in a conventional MDC.

Coupled OsBES links BES and FO units externally through hydraulic connection. An example of a coupled OsBES is to connect an MEC to an FO unit for recovering ammonium from a synthetic wastewater and then applying the recovered ammonium as a draw in the subsequent FO process (Qin and He 2014, Qin et al. 2016b). In an osmotic membrane bioreactor (OMBR) - MFC system, the membrane fouling in the OMBR was alleviated by the MFC treatment, and the electricity generation in the MFC was enhanced due to increased solution conductivity after the OMBR treatment (Hou et al. 2016). FO-based processes have also been studied as pre-treatment before BES. For example, an FO unit containing anaerobic acidification converted complex organic contaminants into short-chain fatty acids and alcohols and also concentrated wastewater, which was then treated in an MFC for electricity generation (Liu et al. 2017). In addition, MDC-FO system can be applied for desalination, and the effluent salinity from MDC-FO system is lower than the maximum contaminant levels of the National Secondary Drinking Water Regulations. Compared to the integration of MDC and RO (ElMekawy et al. 2014), MDC-FO system might have lower energy consumption and lower membrane fouling propensity. 


\subsection{Outline}

This thesis is composed of eleven chapters. The overall goal is to develop the osmotic bioelectrochemcial systems and achieve resource recovery from wastewater towards sustainable wastewater treatment. More specifically, it has the following objectives:

The first objective is to better understand the OsMFCs, which was the first study of BES-FO cooperation. First, the reason why OsMFC has higher current generation than MFC was investigated. Experiments were conducted for comparing with a CEM-MFC and under different operating conditions, and a mathematical model has been developed to predicate the contribution of different factors of internal resistance to current generation with water flux. Second, effects of current generation and electrolyte $\mathrm{pH}$ on reverse salt flux in OsMFC were addressed. As an FO based technology, OsMFCs also encounter reverse salt flux (RSF) that is the backward transport of salt ions across the FO membrane into the treated wastewater. This RSF can reduce water flux, contaminate the treated wastewater, and increase the operational expense, and thus must be properly addressed before any possible applications. In this study, we hypothesized that the unique feature of OsMFCs, electricity generation, could inhibit the reverse salt flux. Third, the ammonium removal in a laboratory OsMFC towards understanding of the effects of current generation and water flux on ammonia removal was investigated. Past OsMFC studies focus on recovery of resources such as electricity and water, but ammonia recovery in OsMFCs has not been investigated. It will be of great interest to accomplish ammonia recovery in OsMFCs, making OsMFCs a promising technology for "NEW recovery" (NEW: nutrient, energy and water). 
The second objective is the development of a mathematical model for ammonia recovery in BES, which could be helpful for further understanding and optimization of ammonia recovery in BES. While a general picture of ammonia recovery using BES is emerging, some issues remain open. For example, the transport of $\mathrm{NH}_{4}{ }^{+}$ions and $\mathrm{NH}_{3}$ is not fully understood despite their essential role in ammonia recovery. In addition, whether $\mathrm{NH}_{4}{ }^{+}$ions move through the $\mathrm{CEM}$ via diffusion or migration is not clear. Another major issue is how the $\mathrm{pH}$ in the anolyte and catholyte is regulated in BES. Resolving the above issues using experimental study alone is difficult due to the challenge in assessing details of all transport processes in the BES. Numerical modeling can address this challenge, but most prior modeling of BES focused on power production and organic removal (Peng et al. 2013, Picioreanu et al. 2007, Ping et al. 2014, Qin et al. 2015, Zeng et al. 2010). In the only comprehensive model for BES-based ammonia recovery, the transport and chemical reactions of major species in BES was studied at a steady state (Dykstra et al. 2014c). The predictions of this model agree qualitatively with experimental observations and they led to critical insight into the effects of current density and membrane properties on the ammonia recovery. However, the model has not been validated by detailed comparison with experiments, and studies based on this model did not cover the issues described above, e.g., the competition of inert ions (i.e., ions that do not react with other species within the system, e.g., the $\mathrm{Na}^{+}$ions) with the $\mathrm{NH}_{4}{ }^{+}$ions for transport across the CEM cannot be studied using steady state models. Here, we have studied the ammonia recovery in a representative BES - microbial electrolysis cells, by integrating experiments with simulations. With this mathematical model, the effects of catholyte aeration rate, external voltage, and external resistance on both ammonia recovery and energy consumption were also investigated. 
The third objective was to develop an MEC-FO system with self-supplied ammonium bicarbonate draw solute for achieving wastewater treatment and recovery. The MEC was to degrade organic compounds (in its anode) and recover ammonia (in the cathode) for preparing draw solute for the following FO treatment, which extracted clean water from the MEC anode effluent. The key parameters including ammonia recovery and regeneration, organic degradation, electricity generation, and water flux were investigated. Then the following study explored the potential of resource recovery from landfill leachate with the MEC-FO system.

The fourth objective was to develop a cost effective alternatives to platinum catalysts in MEC cathode for hydrogen production. In MEC cathode, the kinetic barriers toward proton reduction necessitate the use of catalysts to drive hydrogen formation at appreciable rates and low applied potentials. In this study, a nano- $\mathrm{Ni}(\mathrm{OH})_{2}$ modified cathode was developed by electrodeposition

from a nickel(II) cyclam (cyclam $=1,4,8,11$-tetraazacyclotetradecane) precursor and tested in a bench-scale MEC.

\subsection{Attribution}

Each coauthor is duly credited for his or her contribution to this work, both in their sharing of ideas and technical expertise.

Zhen He, Ph.D. Professor of Civil and Environmental Engineering

(Principal Investigator)

Department of Civil and Environmental Engineering, Virginia Polytechnic Institute and State University. Blacksburg, VA 24061 
Qingyun Ping, Ph.D.

Department of Civil and Environmental Engineering, Virginia Polytechnic Institute and State University. Blacksburg, VA 24061

Coauthor of chapter 3

Yaobin Lu, Ph.D.

Department of Civil and Environmental Engineering, Virginia Polytechnic Institute and State University. Blacksburg, VA 24061

Coauthor of chapter 3

Ibrahim M. Abu-Reesh, D.Sc. Professor of Chemical Engineering

Department of Chemical Engineering, College of Engineering, Qatar University, P.O. Box 2713, Doha, Qatar

Coauthor of chapter $3,4,5$

Erin A. Hynes

Department of Civil, Architectural, and Environmental Engineering, The University of Texas at Austin, Austin, TX 78712, USA

Coauthor of chapter 5 
Ying Liu, Ph.D.

Department of Mechanical Engineering, Virginia Polytechnic Institute and State University.

Blacksburg, VA 24061

Coauthor of chapter 6,7

Shuai Luo

Department of Civil and Environmental Engineering, Virginia Polytechnic Institute and State University. Blacksburg, VA 24061

Coauthor of chapter 6,7

Rui Qiao, Ph.D., Associate Professor

Department of Mechanical Engineering, Virginia Polytechnic Institute and State University.

Blacksburg, VA 24061

Coauthor of chapter 6,7

Hannah Molitor

Department of Civil and Environmental Engineering, University of Wisconsin-Platteville,

Platteville, WI 53818, United States

Coauthor of chapter 9

Brian Brazil 
Waste Management, Gaithersburg, MD 20882, United States

Coauthor of chapter 9

John T. Novak

Department of Civil and Environmental Engineering, Virginia Polytechnic Institute and State University. Blacksburg, VA 24061

Coauthor of chapter 9

William A. Maza, Ph.D.

Department of Chemistry, Virginia Polytechnic Institute and State University, Blacksburg, Virginia 24061, USA

Coauthor of chapter 10

Bethany M. Stratakes

Department of Chemistry, Virginia Polytechnic Institute and State University, Blacksburg, Virginia 24061, USA

Coauthor of chapter 10

Spencer R. Ahrenholtz

Department of Chemistry, Virginia Polytechnic Institute and State University, Blacksburg, Virginia 24061, USA 
Amanda J. Morris, Ph.D., Associate Professor

Department of Chemistry, Virginia Polytechnic Institute and State University, Blacksburg, Virginia 24061, USA

Coauthor of chapter 10 


\title{
Chapter 2 Resource Recovery by Osmotic Bioelectrochemical Systems towards Sustainable Wastewater Treatment
}

(This section has been published as Qin, M. and He, Z.* (2017) Resource Recovery by Osmotic Bioelectrochemical Systems towards Sustainable Wastewater Treatment. Environmental Science: Water Research \& Technology, 3, 583-592.)

\begin{abstract}
Recovering valuable resources from wastewater will transform wastewater management from a treatment focused to sustainability focused strategy, and creates the need for new technology development. An innovative treatment concept - osmotic bioelectrochemical system (OsBES), which is based on cooperation between bioelectrochemical systems (BES) and forward osmosis (FO), has been introduced and studied in the past few years. An OsBES can accomplish simultaneous treatment of wastewater and recovery of resources such as nutrient, energy, and water (NEW). The cooperation can be accomplished in either an internal (integrated OsBES) or external (coupled OsBES) configuration, through a strong synergy between BES and FO. BES can provide draw solute, perform pre-treatment, or reduce reverse salt flux to help with FO operation; while FO can achieve water recovery, enhance current generation, and supply energy sources to BES operation. Given much progress and interest in the OsBES, this paper has reviewed the past studies, described the current status, presented qualitative and quantitative analyses, and discussed the perspectives of the OsBES technology with a focus on NEW recovery from wastewater. The challenges for further researching and developing OsBES have also been identified.
\end{abstract}




\subsection{Introduction}

Increasing global energy demands and the threat of fossil-fuel depletion, environmental pollution, shortage of water and other resources are driving intensive efforts towards developing new technologies for sustainable treatment and utilization of wastewater (Hoffert et al. 2002). Wastewaters contain many valuable sources and among them, three key resources - Nutrient, Energy and Water ("NEW"), are critically important and have a wide range of applications, especially in the food production. NEW recovery has been accomplished in separate processes such as anaerobic digestion (energy), struvite recovery (nutrient) and membrane treatment (water), but each of them has application challenges. For example, anaerobic digestion is usually not applied to low-strength wastewater or small-volume wastewater, struvite recovery is essentially a phosphorus recovery process and ammonia is not efficiently recovered, and pressure-driven membrane treatment is an energy-intensive process. Therefore, there is a strong need for innovative treatment technologies that can accomplish NEW recovery in a resource- and energyefficient way.

Bioelectrochemical system (BES) and forward osmosis (FO) are two state-of-the-art water/wastewater technologies that may play important roles in addressing the challenges of NEW recovery. BES emerges as an attractive treatment concept for energy-efficient wastewater

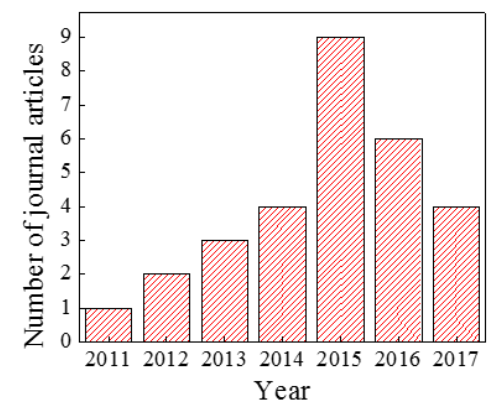

Figure 2.1 Number of published journal articles about OsBES since the first OsBES paper. 
treatment with direct electricity production from wastewater through microbial oxidation of organic contaminants (Wang and Ren 2013). A typical BES contains anode and cathode electrodes, and can be used to generate electricity, produce hydrogen, desalinate saline water, or accomplish other functions. FO is an emerging membrane technology that can extract high-quality water from various water sources (Lutchmiah et al. 2014b). FO allows water to move across a semipermeable membrane from a high water potential zone to a low water potential zone (Shaffer et al. 2015). The individual advantages of BES (e.g., energy recovery and nutrient recovery) and FO (water recovery) intrigue their cooperation to accomplish NEW recovery, and such cooperation would also address the disadvantages of each technology ( $\mathrm{Lu}$ et al. 2014b). For example, FO is concentrating wastewater instead of degrading contaminants, and thus the remaining concentrates from the feed side of FO still require post-treatment (Achilli et al. 2009). Although integration of pressure-drive membrane processes such as ultrafiltration and reverse osmosis membrane into BES can help achieve water recovery (ElMekawy et al. 2014, Yuan and He 2015), it also increases the energy demand by operating membrane processes.

The first study of BES-FO cooperation appeared in 2011 and proposed a new concept of osmotic microbial fuel cells (OsMFCs) (Zhang et al. 2011a). Since then, there have been various studies and efforts to explore the cooperation between BES and FO from different aspects (Figure 2.1). Herein we use osmotic bioelectrochemical system (OsBES) to represent the BES-FO cooperation system. The increasing interest in OsBES and the potentially important role of this technology in NEW recovery create a need for overview of its research and development. This paper aims to provide a timely review of OsBES and summary of the current status of research efforts with a focus on NEW recovery. The basic principles of BES/FO, configurations of OsBES, and mutual 
benefits are described. The challenges and perspectives of NEW recovery by using OsBES have been identified and discussed.

\subsection{Technological Principles}

\subsubsection{Bioelectrochemical System (BES)}

BES relies on the interaction between microorganisms and solid electron acceptors (anode) or donors (cathode) (Logan et al. 2006). In a BES anode, organic compounds in wastewater are oxidized by the exoelectrogens growing on an anode electrode and the generated electrons spontaneously flow from the anode electrode to the cathode electrode, where oxygen is reduced to water (oxygen reduction reaction) (Figure 2.2A) (Rabaey and Verstraete 2005). Ion exchange membranes such as cation exchange membrane (CEM) or anion exchange membrane (AEM) is used as the separator between the anode and the cathode, and there have also been membrane-less BES reactors that still need a separator between the electrodes (Li et al. 2011b). A representative BES is a microbial fuel cell (MFC), which can generate electricity directly from wastewater ( $\mathrm{Li}$ et al. 2014b). MFCs can be modified to accomplish various functions such as hydrogen production, desalination, and heavy metal removal (Logan and Rabaey 2012, Wang and Ren 2013). In a microbial electrolysis cell (MEC), hydrogen gas or other value-added chemicals can be produced with a small amount of external power input (Cheng et al. 2009, Logan et al. 2008a). A microbial desalination cell (MDC) can achieve desalination of saline water in a similar way to that of electrodialysis but with much lower energy demand (Cao et al. 2009). To produce higher-value products such as pure organic compounds, electrons are used by microorganisms in the cathode to achieve microbial electrosysthesis (Rabaey and Rozendal 2010). More details about BES, including the substrates, materials, microbiology, and different system can be found in various 
review papers (Hamelers et al. 2010, Li et al. 2014b, Logan et al. 2006, Rabaey and Verstraete 2005, Schröder et al. 2015, Wang and Ren 2013).

\subsubsection{Forward Osmosis (FO)}

FO is based on the natural phenomenon of osmotic processes, and can extract clean water from wastewater or saline water (Cath et al. 2006, Lutchmiah et al. 2014b). In an FO process, only water molecules can transport across the semi-permeable FO membrane, driven by an osmotic pressure gradient (Figure 2.2B) (Shaffer et al. 2015). Water flux is determined by the osmotic pressure gradient, and draw solutes will play a key role in creating the osmotic pressure difference. Draw solutes such as $\mathrm{NH}_{3} / \mathrm{CO}_{2}$ and magnetic nanoparticles can avoid energy-intensive post-treatment processes like reverse osmosis (Ling et al. 2010, McCutcheon et al. 2005). Fertilizers can also be applied as draw solutes to eliminate the need for regeneration (Phuntsho et al. 2011). Because of small pore radius $(0.25-0.37 \mathrm{~nm})$, FO can achieve effective rejection of a wide range of contaminants (Coday et al. 2014). The lack of external pressure input also results in cost-saving and energy-efficient water extraction and low membrane fouling (Lutchmiah et al. 2014b). FO has been studied to extract reusable water from complex wastewater such as landfill leachate and dewatering of waste activated sludge (Cath et al. 2006). FO can also be applied to produce electric energy in a process called pressure retarded osmosis (PRO), which takes advantage of increased hydraulic pressure due to water expansion of draw solution and uses pressurized flow of water to drive a hydro turbine for power production (Achilli and Childress 2010). The details of FO membrane, limitation, development and potential applications can be found in various review papers (Cath et al. 2006, Chung et al. 2012b, Lutchmiah et al. 2014b, Thompson and Nicoll 2011, Zhao et al. 2012). 


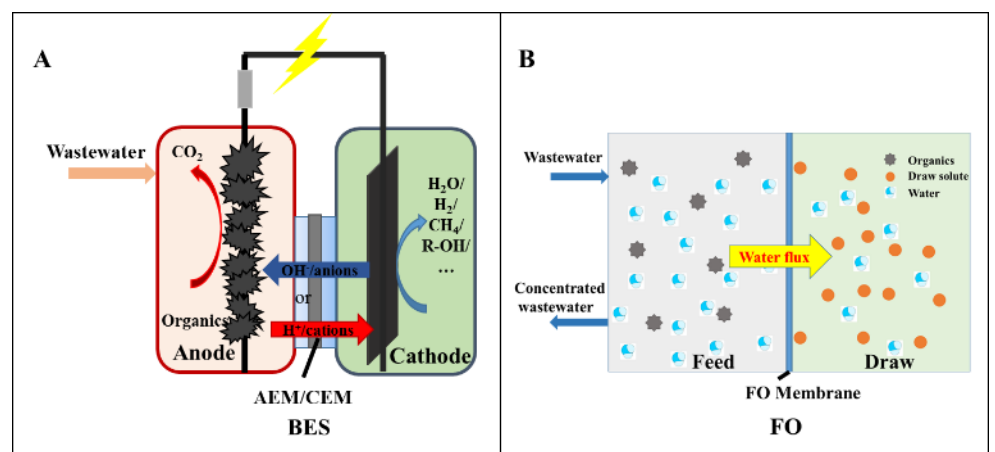

Figure 2.2 Schematics of (A) bioelectrochemical system (BES) and (B) forward osmosis (FO). In this study, we have investigated the relationship between the inter-membrane distance and HRT in a bench-scale MDC with different initial salinities or inter-membrane distances. At the same influent flow rate, six different inter-membrane distances ranging from 0.3 to $2.5 \mathrm{~cm}$ were tested, resulting in different HRTs but the same water production rate $(\mathrm{mL} / \mathrm{min})$. We also analyzed the contributions to conductivity reduction by electric current and water osmosis (water flux into the middle chamber). We studied the effect of different HRTs at the same inter-membrane distances, and the effects of different inter-membrane distances at the same HRTs.

\subsection{Cooperation between BES and FO}

\subsubsection{Configurations}

The cooperation between BES and FO can be accomplished in two ways depending on the location of FO membrane, integrated OsBES (internal cooperation) and coupled OsBES (external cooperation). Integrated OsBES uses FO membrane to replace ion exchange membrane and installs FO membrane within the BES as a separator between an anode and a cathode (Figure 2.3A). The first OsBES was created as an integrated system, in the form of OsMFCs (Zhang et al. 2011a). The anolyte (wastewater) in an OsMFC also acts as the feed solution for the FO process, while a highconcentration catholyte is used as the draw solution. The feasibility of this OsMFC was demonstrated by successful water extraction and bioelectricity generation. The concept of OsMFC 
was further developed to create osmotic microbial electrolysis cells (OsMECs) and microbial desalination cells (OsMDCs) (Lee et al. 2015, Zhang and He 2012). In an OsMEC, external power supply is applied to drive hydrogen evolution reaction in the cathode (Figure 2.3B). In an OsMDC, AEM that separates the anode and the middle compartment is replaced by FO membrane, while CEM is kept between the cathode and the middle compartment (Figure 2.3C). In this way, saline water in the middle compartment would function as a draw solution to extract high quality water from the anolyte, and salts can still be removed driven by electricity generation like that in a conventional MDC.

Coupled OsBES links BES and FO units externally through hydraulic connection (Figure 2.3D). An example of a coupled OsBES is to connect an MEC to an FO unit for recovering ammonium from a synthetic wastewater and then applying the recovered ammonium as a draw in the subsequent FO process (Figure 2.3E) (Qin and He 2014, Qin et al. 2016b). In an osmotic membrane

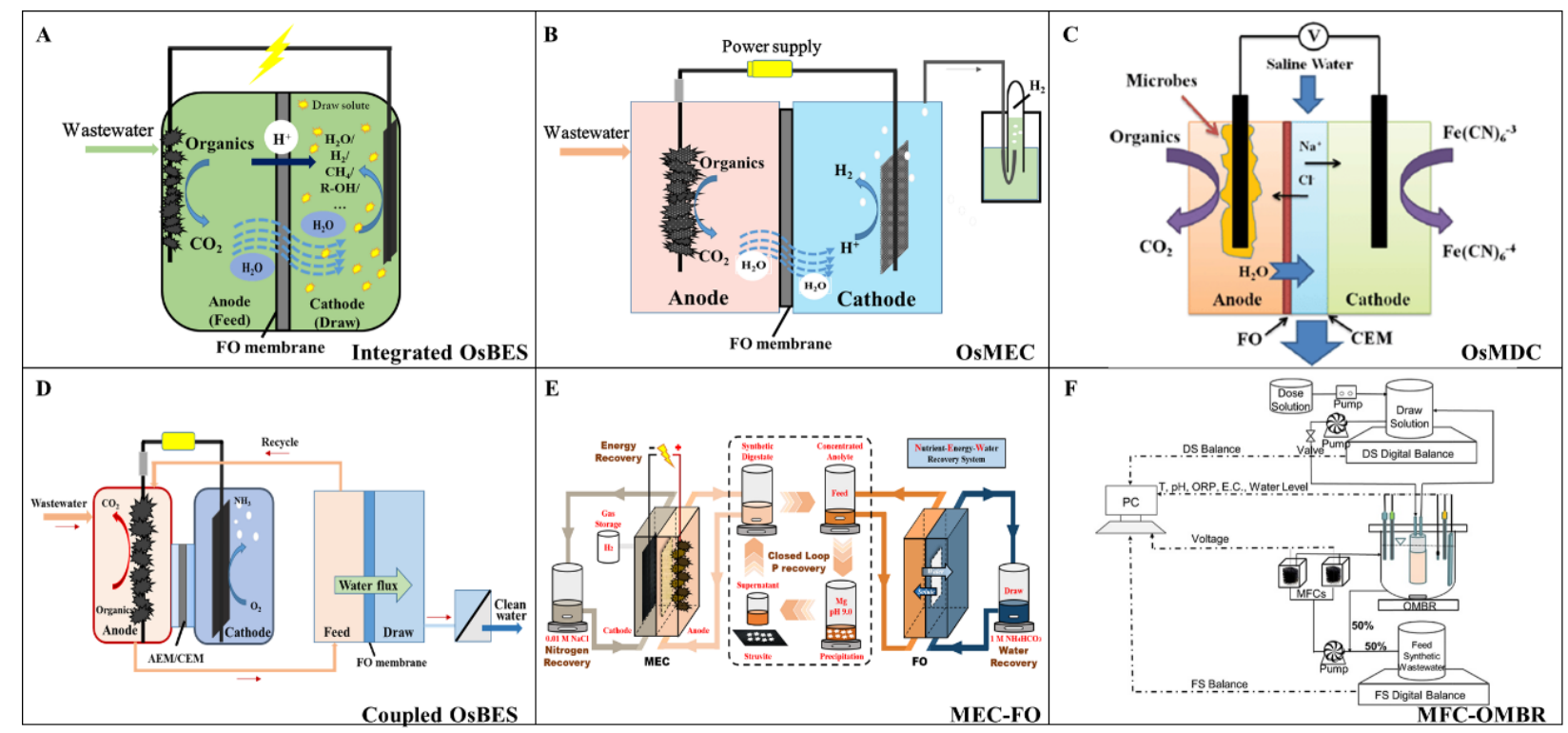

Figure 2.3 Configurations and examples of the cooperation between BES and FO: (A) integrated OsBES; (B) an example of integrated OsBES: OsMFC; (C) another example of integrated OsBES: OsMDC; (D) coupled OsBES; (E) an example of coupled OsBES: MECFO; and (F) another example of coupled OsBES: MFC-OMBR. 
bioreactor (OMBR) - MFC system, the membrane fouling in the OMBR was alleviated by the MFC treatment, and the electricity generation in the MFC was enhanced due to increased solution conductivity after the OMBR treatment (Figure 2.3F) (Hou et al. 2016). FO-based processes have also been studied as pre-treatment before BES. For example, an FO unit containing anaerobic acidification converted complex organic contaminants into short-chain fatty acids and alcohols and also concentrated wastewater, which was then treated in an MFC for electricity generation (Liu et al. 2017). In addition, MDC-FO system can be applied for desalination, and the effluent salinity from MDC-FO system is lower than the maximum contaminant levels of the National Secondary Drinking Water Regulations. Compared to the integration of MDC and RO (ElMekawy et al. 2014), MDC-FO system might have lower energy consumption and lower membrane fouling propensity.

\subsubsection{Mutual Benefits}

The cooperation between BES and FO, either integrated or coupled, can accomplish the recovery of valuable resources such as energy, water, and nutrient (more details in the section 2.4). This cooperation can also create mutual benefits that strengthen each of the unit in this system (Figure 2.4). As mentioned earlier, an OMBR and an MFC could benefit each other in terms of enhancing electricity generation and reducing membrane fouling (Hou et al. 2016). Enhancing electricity

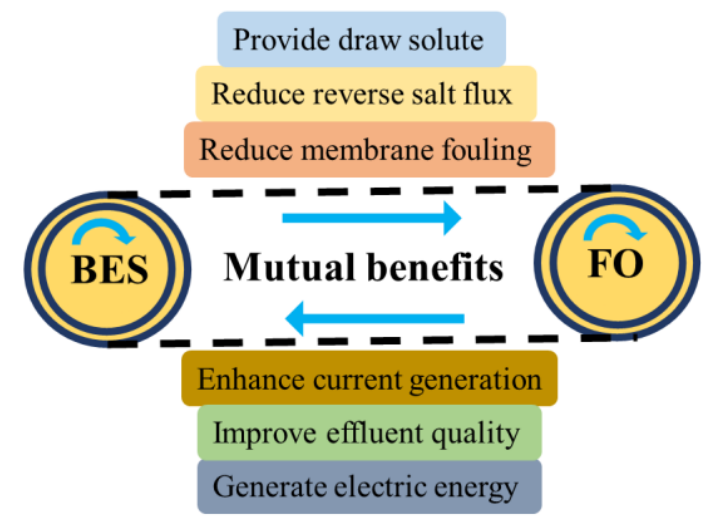

Figure 2.4 Mutual benefits between BES and FO in OsBES. 
generation by FO has already been demonstrated in the first OsMFC study, which showed that the OsMFC generated more electricity than a regular MFC containing CEM (Zhang et al. 2011a). The reason for the higher current generation in the integrated OsBES was interpreted from two aspects: ion transport and system resistance. Water flux, a unique feature of the OsBES (compared to the regular BES), can promote ion transport from the anolyte to the catholyte, thereby reducing electrolyte resistance (Zhang et al. 2011a, Zhu et al. 2015). The lower system resistance of the OsBES also benefits from the concentrated anolyte (due to water flux) and lower FO membrane resistance. A mathematical model has been developed to explain the low internal resistance of an OsMFC, and the results revealed that a lower resistance of FO membrane than that of CEM played an important role in decreasing overall internal resistance of the OsMFC, thereby resulting in more electricity generation (Qin et al. 2015). A special FO reactor - pressure retarded osmosis (PRO), which can generate electric energy from hydraulic pressure due to water flux, was employed to provide external power to an MEC for hydrogen production (Yuan et al. 2015b). A mathematical model predicated that at $0.9 \mathrm{~V}$, the energy requirement by the MEC would reach the maximum energy production by the PRO.

Several studies have found that BES can also benefit FO. A key issue with FO operation is reverse solute flux (RSF), which causes the loss of draw solute and creates problems of reduced water flux and post-disposal of the accumulated salts in the feed. It was reported that bioelectricity generation in an OsMFC could significantly reduce RSF. When the total Coulomb production increased from zero (open circuit) to $311 \mathrm{C}$, RSF was reduced from $16.3 \pm 2.8$ to $3.9 \pm 0.7 \mathrm{gMH}$ (Qin et al. 2016a). Electrically-driven migration was identified to be a more important factor to RSF reduction than diffusion-driven migration. BES can also provide draw solute to FO. In an MEC-FO system, the 
MEC recovered ammonium from a high-strength synthetic wastewater and the recovered ammonium was then applied as a draw solute (in the form of $\mathrm{NH}_{4} \mathrm{HCO}_{3}$ ) in the following $\mathrm{FO}$ unit that extracted water from the MEC effluent (Qin and He 2014). This system has also been investigated for recovering ammonium from landfill leachate and reducing the volume of the treated leachate through FO using recovered ammonium (Qin et al. 2016b).

\subsection{NEW Recovery in OsBES}

\subsubsection{Energy Recovery}

Energy recovery in an OsBES is mainly from the BES-based unit, because BES can produce electricity or hydrogen gas from organic wastes. Energy can be described by using normalized energy recovery (NER), which is energy production per either the volume of the treated wastewater or the removed organics (e.g., chemical oxygen demand - COD) (Ge et al. 2013a). Like BES studies, many OsBES studies do not report energy data; only power density data are presented. To better understand BES/OsBES performance and identify their application niches, energy data must be included (He 2017). Table 2.1 summarizes the studies that either reported energy data or provided sufficient information that we could use to estimate energy recovery. It should be noted that we tend not to make comparison between different studies, because of the difference in reactor configurations and operation. One can see that the NER of the OsBES varies from 0.001 to 0.1

$\mathrm{kW} \mathrm{h} \mathrm{m}{ }^{-3}$ wastewater, and such variation is related to the different OsBES configurations, operation conditions, and organic loading rates. 
Table 2.1 Summary of the OsBES studies for energy recovery.

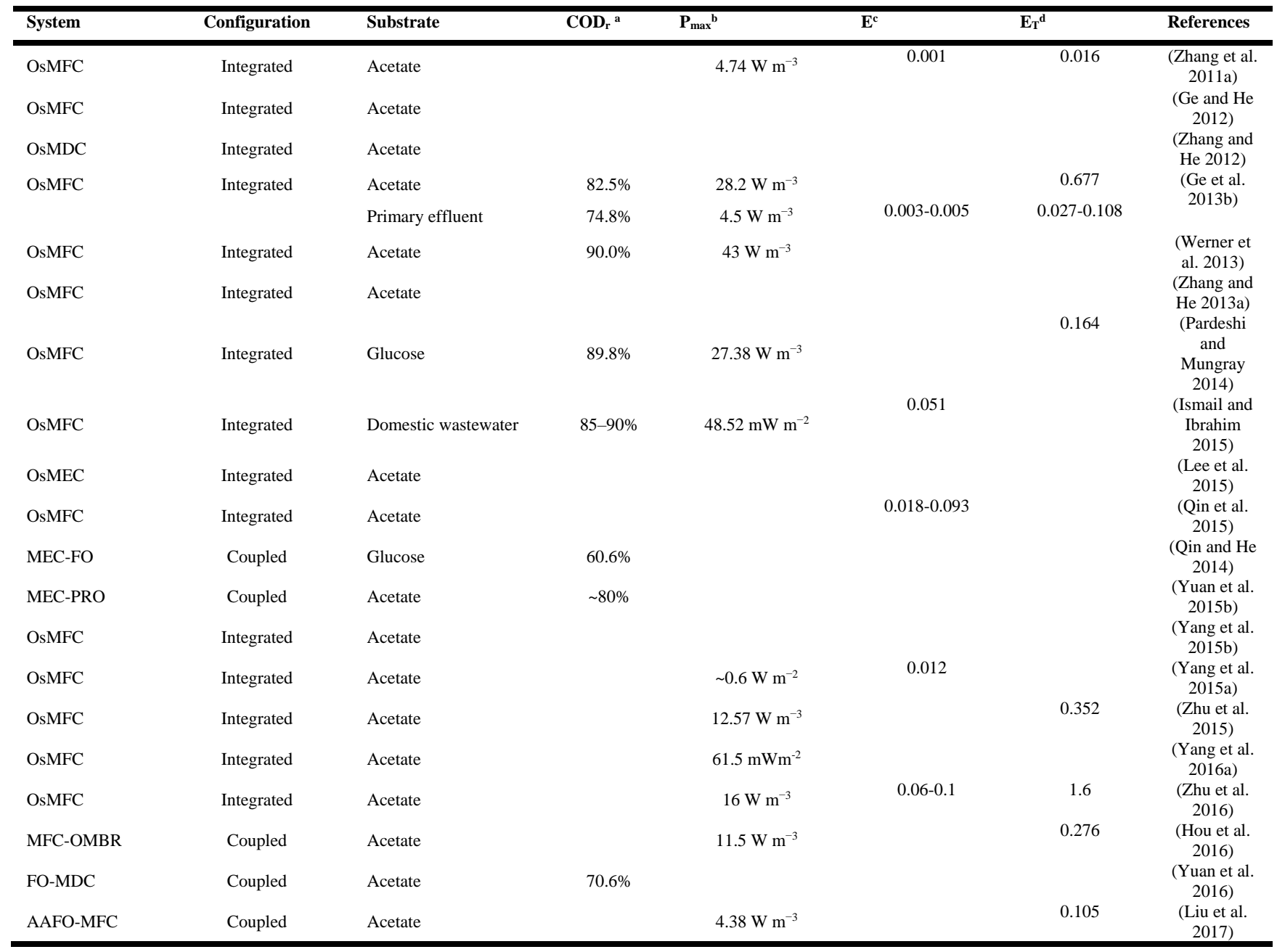

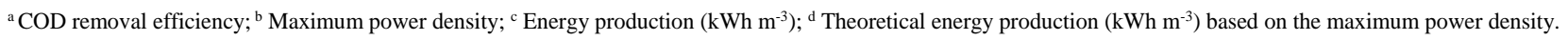

Although OsBES can generate energy, the data of energy recovery alone is not particularly useful because the operation of an OsBES is also consuming energy at the same time. If the recovered energy is smaller than the consumed energy, external energy input will still be needed to operate an OsBES. Thus, establishing an energy balance for an OsBES is cortically important to understand the energy benefit. Unfortunately, even fewer studies reported energy consumption of their OsBES. Figure 2.5 shows the examples of energy recovery and consumption in an integrated 
OsBES and a coupled OsBES. The OsMFC generated $0.093 \mathrm{kWh} \mathrm{m}^{-3}$ treated water as electricity but consumed $0.81 \mathrm{kWh} \mathrm{m}^{-3}$ treated water; $97.4 \%$ of energy consumption was due to the catholyte aeration (Figure 2.5A). Overall, the OsMFC had a negative energy balance of $0.717 \mathrm{kWh} \mathrm{m}^{-3}$.(Qin et al. 2015) In the coupled OsBES (MEC-FO), energy was recovered as hydrogen gas, which could be converted to a NER of $0.36 \mathrm{kWh} \mathrm{m}^{-3}$ treated water (Figure 2.5B) (Zou et al. 2017). The energy consumption of this system was $1.26 \mathrm{kWh} \mathrm{m}^{-3}$ treated water, $94.4 \%$ by the MEC external power supply, $4.8 \%$ due to the MEC electrolyte recirculation, and $0.8 \%$ by the FO process. The generated hydrogen gas could supply up to $28.7 \pm 1.5 \%$ of total energy demand.

Despite negative energy balances in both of the above examples, those analyses have helped us to better understand the energy picture of an OsBES towards its optimization. Some OsBES were not operated under the condition of the maximum power output (which would lead to more energy production). As shown in Table 1, the theoretical energy generation calculated from the maximum current density can be much higher than that calculated from the actual current generation. Thus, optimizing power output will be an effective approach to improve energy recovery. There are also

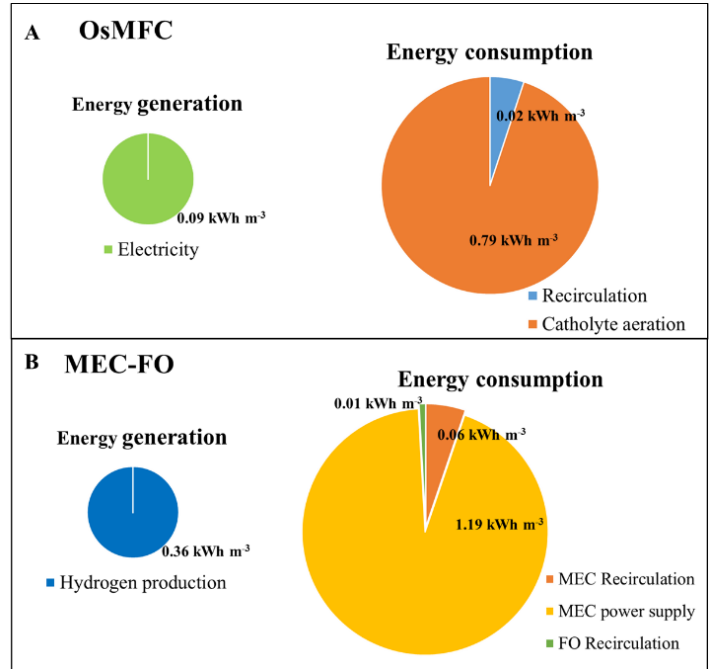

Figure 2.5 Examples of energy recovery and consumption in the OsBES: (A) an OsMFC treating synthetic municipal wastewater; (B) an MEC-FO system treating synthetic sidestream centrate. 
methods to reduce energy consumption towards a neutral or even positive energy balance. For example, aeration was identified as the dominant energy consumer in the OsMFC (Figure 2.5A), and replacing active aeration with passive aeration (e.g., air cathode BES) or precisely controlled aeration such as membrane aeration can reduce related energy consumption (Werner et al. 2013, Yang et al. 2016c). Conventional air cathode will not be suitable for an OsBES, because a catholyte is required to act as a draw solution; a flowing-over cathode may provide passive aeration to an

OsBES but this needs experimental investigation (Clauwaert et al. 2009). In the MEC-FO system (Figure 2.5B), external power supply was the main energy consumer, and this consumption may be reduced by decreasing the external voltage or using renewable source of energy. For example, in the previously mentioned MEC-PRO system (a coupled OsBES), the external voltage for the MEC was provided by the PRO (Yuan et al. 2015b).

\subsubsection{Water Recovery}

Extraction of high-quality water via forward osmosis is a distinct feature of an OsBES compared with a conventional BES. The first OsBES study has provided a strong experimental evidence that the OsMFC achieved water flux of $3.94 \pm 0.22 \mathrm{LMH}$ (liters per square meter FO membrane per hour) with a $116-\mathrm{g} \mathrm{NaCl} \mathrm{L}^{-1}$ catholyte, while there was no obvious water flux in a conventional MFC (Figure 2.6A) (Zhang et al. 2011a). More than 50\% of the treated wastewater could be extracted as clean water from various types of wastewater (Ge et al. 2013b, Ismail and Ibrahim 2015). That is, by using the OsBES technology, half of the wastewater can be potentially reused, instead of being discharged.

A key factor to water recovery in the OsBES is draw solute, and an ideal draw solute should possess a certain ability of $\mathrm{pH}$ buffering (in response to $\mathrm{pH}$ increase due to cathodic reduction 
reactions) and have a high conductivity (creating a high osmotic pressure) (Ge and He 2012). In addition, low cost and non-fouling formation are also important features. Several representative draw solutions have been examined in an OsMFC for water flux, and phosphate buffer saline resulted in the best performance; although glucose could act as a draw solute, it led to little current generation in the OsMFC because of no conductivity (Ge and He 2012). Table 2.2 summarizes the studies with various draw solutes, and one can see that $\mathrm{NaCl}$ is the most commonly used draw solute, which could provide both osmotic pressure gradient and catholyte conductivity. This encourages the exploration of using seawater as a catholyte in the OsMFC, and the diluted seawater (due to water recovery) can be further desalinated (Zhang and He 2013a). In the case of seawater draw solute, there is no need to regenerate draw solute, greatly saving the operational cost. Other non-regenerated draw solutes include fertilizers (Phuntsho et al. 2011, Phuntsho et al. 2012). In many other cases, regeneration of draw solutes is necessary and then low-energy regeneration will be critical to maintain a low energy demand. Examples of such draw solutes include ammonium biocarbonate (which can be regenerated by low heat), hydrogels (regenerated under a range of stimuli, such as temperature, light, pressure, and sound) and magnetic nanoparticles nanoparticles (regenerated by magnetic field) (Akther et al. 2015, Li et al. 2011a, Ling et al. 2010, McCutcheon et al. 2005, Shaffer et al. 2015). 
Other factors that affect water recovery include membrane fouling and liquid recirculation rate. Fouling is an inevitable phenomenon in a membrane-based process. As water permeates the membrane, foulants accumulate on the membrane surface, forming a cake layer, which creates hydraulic resistance and reduce the driving force for water recovery (Hoek and Elimelech 2003). It has been shown that that fouled membrane could significantly reduce water flux compared to the pristine membrane in an OsMFC (Figure 2.6B) (Zhu et al. 2016). Periodic cleaning of FO membrane, regeneration of catholyte and replacement of anolyte could be effective for relieving the membrane fouling and achieving continuing water recovery. Typical FO operations are performed with a very high recirculation rate that creates a shear force to hinder foulants from accumulating on the membrane surface and reduce the external concentration polarization (Boo et al. 2013). The liquid recirculation rates in an OsBES are usually much lower than that of typical FO (cross-flow velocity: $0.01-0.02 \mathrm{~m} \mathrm{~s}^{-1}$ in the OsBES vs. $10-30 \mathrm{~m} \mathrm{~s}^{-1}$ in the FO) (Lee et al. 2010, Yuan et al. 2015b, Zhang et al. 2011a) and in the presence of electrodes and biofilm, a high
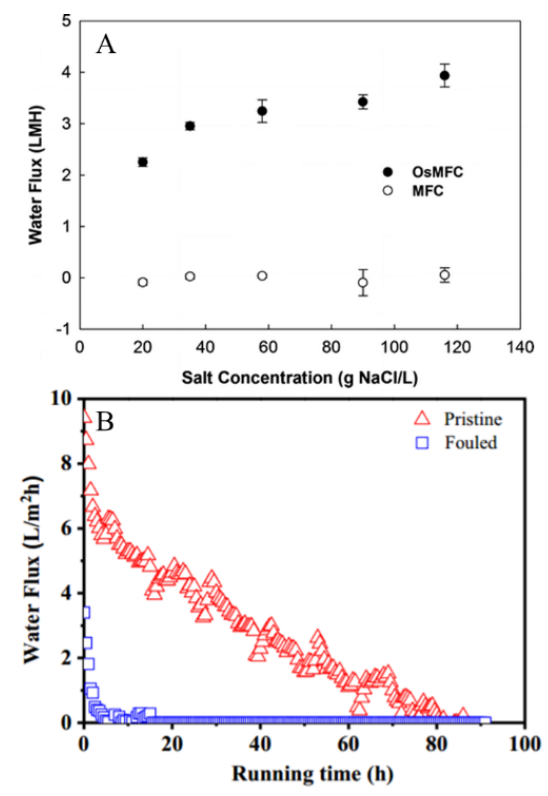

Figure 2.6 Water recovery in the OsBES: (A) an OsMFC generates higher current than a conventional MFC at different concentrations of $\mathrm{NaCl}$ draw solution; and (B) fouled FO membrane greatly reduces water flux compared to pristine membrane. 


\section{Table 2.2 Summary of the OsBES studies for water recovery}

\begin{tabular}{|c|c|c|c|c|c|c|}
\hline System & Configuration & Membrane type & Draw solute & $\mathrm{DS}^{\mathrm{a}}$ & Water flux ${ }^{b}$ & References \\
\hline OsMFC & Integrated & CTA & $\mathrm{NaCl}$ & $20-116 \mathrm{~g} \mathrm{~L}^{-1}$ & $1.2-2.8$ & (Zhang et al. 2011a) \\
\hline \multirow{4}{*}{ OsMFC } & Integrated & CTA & $\mathrm{NaCl}$ & $1 \mathrm{M}$ & 1.82 & (Ge and $\mathrm{He} 2012$ ) \\
\hline & & & $\mathrm{CaCl}_{2}$ & $1 \mathrm{M}$ & 0.56 & \\
\hline & & & PBS buffer & $1 \mathrm{M}$ & 2.42 & \\
\hline & & & Glucose & $1 \mathrm{M}$ & 1.82 & \\
\hline OsMDC & Integrated & CTA & $\mathrm{NaCl}$ & $5-20 \mathrm{~g} \mathrm{~L}^{-1}$ & $0.29-0.69$ & (Zhang and He 2012) \\
\hline OsMFC & Integrated & CTA & $\mathrm{NaCl}$ & $2 \mathrm{M}$ & $1.11-1.49$ & (Ge et al. 2013b) \\
\hline OsMFC & Integrated & CTA & $\mathrm{NaCl}$ & $35 \mathrm{~g} \mathrm{~L}^{-1}$ & $1.5-5.8$ & (Werner et al. 2013) \\
\hline OsMFC & Integrated & CTA & $\mathrm{NaCl}$ & $10-50 \mathrm{~g} \mathrm{~L}^{-1}$ & $0.3-0.8$ & $\begin{array}{c}\text { (Zhang and } \mathrm{He} \\
\text { 2013a) }\end{array}$ \\
\hline OsMFC & Integrated & Chitosan $^{c}+$ PAAc & $\mathrm{NaCl}$ & $2 \mathrm{M}$ & $18.4-34.4$ & $\begin{array}{c}\text { (Pardeshi and } \\
\text { Mungray 2014) }\end{array}$ \\
\hline OsMFC & Integrated & CTA & Oil produced water & & $1.8-4.1$ & $\begin{array}{l}\text { (Ismail and Ibrahim } \\
\text { 2015) }\end{array}$ \\
\hline OsMEC & Integrated & CTA & PBS & $24 \mathrm{~g} \mathrm{~L}^{-1}$ & & (Lee et al. 2015) \\
\hline OsMFC & Integrated & CTA & $\mathrm{NaCl}$ & $35 \mathrm{~g} \mathrm{~L}^{-1}$ & 2.3 & (Qin et al. 2015) \\
\hline MEC-FO & Coupled & CTA & $\mathrm{NH}_{4} \mathrm{HCO}_{3}$ & $0.8 \mathrm{M}$ & 3.0 & (Qin and He 2014) \\
\hline MEC-PRO & Coupled & CTA & $\mathrm{NaCl}$ & $0.1-2.0 \mathrm{M}$ & $0.5-1$ & (Yuan et al. 2015b) \\
\hline OsMFC & Integrated & CTA & $\mathrm{NaCl}$ & $35 \mathrm{~g} \mathrm{~L}^{-1}$ & $1.3-4.1$ & (Yang et al. 2015b) \\
\hline OsMFC & Integrated & CTA & $\mathrm{NaCl}$ & $35 \mathrm{~g} \mathrm{~L}^{-1}$ & & (Yang et al. 2015a) \\
\hline OsMFC & Integrated & TFC & $\mathrm{NaCl}$ & $1 \mathrm{M}$ & $1-6$ & (Zhu et al. 2015) \\
\hline OsMFC & Integrated & СТA & $\mathrm{NaCl}$ & $35 \mathrm{~g} \mathrm{~L}^{-1}$ & 2.33 & (Yang et al. 2016a) \\
\hline OsMFC & Integrated & TFC & $\mathrm{NaCl}$ & $1 \mathrm{M}$ & $3.25 \pm 0.60$ & (Zhu et al. 2016) \\
\hline MFC-OMBR & Coupled & TFC & $\mathrm{NaCl}$ & $0.5 \mathrm{M}$ & $2.0-12.0$ & (Hou et al. 2016) \\
\hline FO-MDC & Coupled & CTA & $\mathrm{NaCl}$ & $35 \mathrm{~g} \mathrm{~L}^{-1}$ & $0.64-0.99$ & (Yuan et al. 2016) \\
\hline AAFO-MFC & Coupled & TFC & $\mathrm{NaCl}$ & $5 \mathrm{M}$ & $2.33-5.62$ & (Liu et al. 2017) \\
\hline
\end{tabular}

a Draw solution concentration; ${ }^{\mathrm{b}}$ in the unit "LMH"; c [CH; polycation]

recirculation rate may not be possible or could wash biofilm off the electrodes. Therefore, it is expected that water recovery in OsBES will be lower than that of traditional FO systems.

\subsubsection{Nutrient Recovery}

Both BES and FO have been studied for nutrient removal and recovery. In BES, ammonia nitrogen can be removed and recovered driven by electricity generation (Kelly and He 2014), and phosphorous is recovered through precipitation by taking advantage of high $\mathrm{pH}$ as a result of cathodic reduction reactions (Zang et al. 2012). FO can concentrate the target compounds such as phosphate in wastewater and thus increase the probability of recovery (Ansari et al. 2017). As an inheritor of both BES and FO, the OsBES is expected to have a function of nutrient recovery. 
Surprisingly the related studies are very limited. A recent study reported, for the first time, that an OsMFC could effectively remove ammonium nitrogen, although the recovery was not investigated (Qin et al. 2017). It was found that current generation and water flux were two key factors to drive ammonium movement across FO membrane. Current generation could greatly enhance the removal efficiency from $40.7 \pm 2.4 \%$ to $85.3 \pm 3.5 \%$ when the current density increased from 0 to $1.8 \pm 0.1 \mathrm{~A} \mathrm{~m}^{-2}$. Water flux could facilitate the ammonium removal from wastewater. In a coupled OsBES (MEC+FO), the recovered ammonia in the MEC was applied as a draw solute for water recovery in the FO process (Qin and He 2014), and this system was further studied to include phosphorus recovery as struvite $\left(\mathrm{MgNH}_{4} \mathrm{PO}_{4} \cdot 6 \mathrm{H}_{2} \mathrm{O}\right)$ (Zou et al. 2017). In a closed-loop mode, this coupled OsBES achieved the recovery of $54.2 \pm 1.9 \%$ of water, $99.7 \pm 13.0 \%$ of net ammonium nitrogen, and $79.5 \pm 0.5 \%$ of phosphorus as struvite (Zou et al. 2017). Ammonium loss primarily from RSF in the FO was fully compensated by the reclaimed ammonium under extended $\mathrm{N}_{2}$ stripping. In another coupled OsBES (MRC-OMBR), both nitrogen and phosphorus was recovered by taking advantage of the electrical potential generated across the anode and the cathode in the MRC and the charged ions such as $\mathrm{NH}_{4}{ }^{+}, \mathrm{PO}_{4}{ }^{3-}$ and salts were extracted from the OMBR bulk solution. This system could effectively recover up to $65 \% \mathrm{PO}_{4}{ }^{3-}$ and $45 \% \mathrm{NH}_{4}{ }^{+}$from the synthetic wastewater.

\subsection{Perspectives and Challenges}

\subsubsection{Potential Applications}

An OsBES may be applied to recover valuable resources with simultaneous treatment of various wastewaters including both low strength (e.g., municipal wastewater) and high strength (e.g., side stream, food/beverage wastewater, livestock wastewater, and landfill leachate). However, the strategies and goal of the applications will differ, because of different characteristics of 
wastewaters. The application to treat low strength wastewater will need to focus on energyefficient treatment and water recovery. Energy recovery from low strength wastewater is still important, but its primary goal will be to help achieve energy-neutral treatment. Nutrient recovery may not be feasible, due to very low concentrations of both nitrogen and phosphorus in low strength wastewater. Treating low strength wastewater will require a large volume of the reactor to obtain a low hydraulic retention time (HRT), thereby creating more challenge in system scaling up. But an OsBES can achieve very good treatment efficiency with low strength wastewater. For example, an OsMFC achieved COD removal efficiency of $82.5 \pm 9.0 \%$ and recovered up to $70 \%$ of water (Ge et al. 2013b). An MFC-OMBR system exhibited excellent removal of organic (>95\%) and phosphorus (>99\%) and could maintain an effluent COD concentration below $20 \mathrm{mg} \mathrm{L}^{-1}$ (Hou et al. 2016).

High-strength wastewater contains high concentrations of organic matters and nutrients, and will be suitable for simultaneous NEW recovery. A prior study has examined the NEW recovery from a synthetic sidestream and achieved effective recovery of water, ammonium nitrogen, and phosphorus (Zou et al. 2017). A challenge of treating high strength wastewater by using an OsBES is the potential competition with anaerobic digestion, which is a mature and efficient technology for biogas production. Some high strength wastewaters such as side stream contain a large amount of solids that may not be suitable for treatment in a system containing electrodes like an OsBES. Thus, synergistic linkage between anaerobic digestion and OsBES may benefit each other: in such a linkage, anaerobic digestion treats high-solid wastewater and generates biogas, and the OsBES further treats the effluent from digestion for nutrient recovery and reducing the volume of wastewater discharge (via water recovery). The ammonia recovery in an OsBES could also 
counteract the ammonia inhibition for anaerobic digestion process (Zhang and Angelidaki 2015a, b).

The application of the coupled OsBES will need to address a key issue of system coordination between its BES unit and FO unit. BES is a biological process and tends to be slow, while FO is a physical process and usually fast. The difference in the treatment speed between the two creates the issue of coordinating the treatment capacities. In a previous study of a coupled OsBES (MECFO), the HRT of an MEC that was used for energy and ammonia recovery was $48 \mathrm{~h}$ while the FO unit required only $5 \mathrm{~h}$ to complete water recovery (Qin and He 2014). As a result, the FO unit was on standby most of the operation time and its treatment capacity would be wasted. Such discrepancy may be solved by increasing the treatment speed of the BES unit (e.g., through optimizing BES operation and materials), or by reducing the size of the FO unit so that a continuous supply of BES effluent can be achieved.

\subsubsection{Recovery of Other Resources}

In addition to NEW, wastewaters also contain other types of resources or can be used to create value-added resources. Some wastewaters such as metal industry wastewater and landfill leachate have high concentrations of various metal ions, and recovering those metals will avoid environmental pollution and reduce the raw material consumption for producing metals. BES offers an attractive approach for both oxidation and reduction reaction oriented processes and therefore, can be used for effective metal recovery (Wang and Ren 2014). Metals such as Au (III), $\mathrm{V}(\mathrm{V}), \mathrm{Cr}(\mathrm{VI}), \mathrm{Ag}(\mathrm{I}), \mathrm{Cu}(\mathrm{II}), \mathrm{Fe}$ (III), $\mathrm{Hg}$ (II), $\mathrm{Pb}$ (II), Cd (II), and Zn (II), can be reduced on the cathode of a BES and precipitate for further recovery. If metal recovery occurs outside a BES, a low catholyte $\mathrm{pH}$ will be necessary to keep metal in the dissolved form and such $\mathrm{pH}$ can also 
benefit the cathodic reduction reactions. Although there have not been any studies of using an OsBES for metal recovery, there may be two benefits with the OsBES approach: first, water flux can facilitate ion transport and thus promote metal ion movement from the anolyte into the catholyte for separation and recovery; and second, the higher current generation in OsBES could potentially enhance the metal reduction in the cathode for subsequent recovery. The value-added

biochemical products, such as methane, alcohols, acetate, and hydrogen peroxide, can be generated in BES and thus may also be produced in OsBES (Kumar et al. 2017). Further separation of those products from draw solutes will be a challenge to address.

\subsubsection{Membrane Fouling}

Although membrane fouling in an FO process is generally less severe than that in pressure-driven membrane processes, it is still an inevitable phenomenon in an OsBES, and can lead to an additional hydraulic resistance that reduces osmotic pressure and water recovery, thereby increasing the capital and operational costs. When treating wastewater, the organic fouling due to the presence of organic matters and microorganisms can become significant (Zhao et al. 2011), and in this aspect, a coupled OsBES may be more advantageous over an integrated OsBES, because the BES unit in the coupled OsBES acts as a pre-treatment step and reduces organic contents in the liquid stream before it enters the FO unit. When fouling occurs, backwash is a commonly used method to remove foulants and retrieve the FO performance. Membrane fouling in FO can be almost fully reversible by backwash, due to the loose fouling layer on the membrane surface in the absence of external pressure (Lee et al. 2010). More severe fouling would require chemical cleaning, either in situ or ex situ. No matter which cleaning method, the microbial community in the anode of an integrated OsBES would be adversely affected, for example by a shock from a high salinity solution or chemical toxicity. To address this problem, membrane 
fabrication and modification must be advanced to reduce membrane fouling and cleaning requirement. A prior study has examined an FO membrane modified with silver nanoparticles $(\mathrm{nAg})$ in an OsMFC and observed a substantial reduction in biofouling as well as further improvement in current generation (Yang et al. 2016a). Fouling alters the membrane properties and in some cases, it may benefit energy recovery in an OsBES. It was found that the current generation of an OsMFC was increased by $34 \%$ upon membrane fouling without water flux (Zhu et al. 2016). This is because that the fouled FO membrane showed significantly higher flux of protons and other ions than a pristine membrane, resulting in a lower internal resistance and higher current generation.

\subsubsection{System scaling up}

Development of the OsBES technology must advance system scaling up, and this will be built on the ongoing scaling up efforts for both BES and FO. Scaling up FO is relatively easy and straightforward, because of its simple structure. There have been several pilot FO systems developed for extracting water from domestic wastewater, fracking wastewater and mine-impaired groundwater (Hancock et al. 2011b, McGinnis et al. 2013, Phuntsho et al. 2016). Thus, the key challenge to scaling up an OsBES will lie in the BES part. There have been a number of reports of BES scaling up, such as MFCs, MECs and MDCs (Cusick et al. 2011, Feng et al. 2014, Wu et al. 2016, Zhang and He 2015), and those will provide valuable experiences to scaling up OsBES. An effective step to transform laboratory systems to pilot/full systems is to establish a transitionalscale system, which has a significantly larger size than bench systems but smaller than pilot/full systems. Such a transitional system possesses some features of both bench and pilot/full systems, and can be a great platform for further research. For example, a 200-L MFC system was developed as a transitional-scale system (much larger than bench MFCs that are usually no more than $1 \mathrm{~L}$ but 
smaller than typical pilot systems of >3,000 L) for treating domestic wastewater (Ge et al. 2015). Scaling up the integrated OsBES will largely benefit from BES scaling up efforts. Mathematical modeling can be a powerful tool to help develop transitional-scale systems based on the results of bench systems (Luo et al. 2016). A key objective for studying the transitional-scale systems is to perform techno-economic analysis, which will reveal both technical and economical feasibility of the OsBES technology. Involving both membranes and electrodes in an OsBES can result in a high capital cost; however, if an appropriate application niche is identified, there could be the potential need for this technology. In the previously mentioned study of 200-L MFC system, the initial analysis of the capital cost indicated that this system could be more competitive in the market of small-scale wastewater treatment (e.g., $<40 \mathrm{~m}^{3}$ day $^{-1}$ ) (Ge et al. 2015). Rapid progress in material science will lead to the reduction of the cost associated with membranes and electrodes.

\subsection{Conclusions}

The cooperation of BES and FO creates an innovative treatment system, which takes advantage of both technologies and can accomplish the recovery of valuable resources such as energy, water, and nutrient from various wastewaters. The cooperation, through either internal or external connection, also generates a strong synergy between BES and FO and helps overcome some inherent issues of each technology. The research of OsBES is still in an infancy stage, but great promise in resource recovery and progress of both BES and FO as individual technology will accelerate the development of the OsBES technology. More efforts must be invested to identify application niches, understand energy issues, alleviate membrane fouling, and scale up OsBES to a transitional stage. 


\title{
Chapter 3 Understanding Electricity Generation in Osmotic Microbial Fuel Cells through Integrated Experimental Investigation and Mathematical
}

\section{Modeling}

(This section has been published as Qin, M., Ping, Q., Lu, Y., Abu-Reesh, I. M., \& He, Z.* (2015). Understanding Electricity Generation in Osmotic Microbial Fuel Cells through Integrated Experimental Investigation and Mathematical Modeling. Bioresource Technology, 195, 194-201.)

\begin{abstract}
Osmotic microbial fuel cells (OsMFCs) are a new type of MFCs with integrating forward osmosis (FO). However, it is not well understood why electricity generation is improved in OsMFCs compared to regular MFCs. Herein, an approach integrating experimental investigation and mathematical model was adopted to address the question. Both an OsMFC and an MFC achieved similar organic removal efficiency, but the OsMFC generated higher current than the MFC with or without water flux, resulting from the lower resistance of $\mathrm{FO}$ membrane. Combining $\mathrm{NaCl}$ and glucose as a catholyte demonstrated that the catholyte conductivity affected the electricity generation in the OsMFC. A mathematical model of OsMFCs was developed and validated with the experimental data. The model predicated the variation of internal resistance with increasing water flux, and confirmed the importance of membrane resistance. Increasing water flux with higher catholyte conductivity could decrease the membrane resistance.
\end{abstract}




\subsection{Introduction}

Wastewater is considered as a valuable source of energy and other resources. Sustainable wastewater treatment aims to remove contaminants in a more energy-efficient way with resource recovery (McCarty et al. 2011). Microbial fuel cells (MFCs) emerge as a promising technology for sustainable wastewater treatment with direct electricity production from wastewater through microbial oxidation of organic contaminants (Li et al. 2014a). Synergistic integration of forward osmosis (FO) into MFCs create osmotic microbial fuel cells (OsMFCs) (Zhang et al. 2011a), which possess a new function of recovering high-quality water and thus benefit water reclamation towards reducing water footprint. In a FO process, water can move across a semipermeable membrane from a high water potential zone to a low water potential zone (Shaffer et al. 2015). The driving force is provided by a salt gradient that creates an osmotic pressure. Compared to other membrane-based processes, FO is less energy intensive and low membrane fouling (Lutchmiah et al. 2014a).

An OsMFC inherits the advantages of both MFCs and FO systems, generating electricity from the organics by exoelectrogens and extracting clean water from wastewater by FO membrane (Zhang et al. 2011a). This represents new synergy between bioelectrochemical systems and FO, and can be extended to microbial electrolysis cells (MECs) and microbial desalination cells (MDCs) for various purposes (Lu et al. 2014b). Coupling the MFC (or MDC, MEC) with FO could achieve wastewater treatment, energy recovery, and resource recovery (such as clean water, nutrients) in one system (Qin and He 2014, Yuan et al. 2015a, Zhang and He 2013a). This promising combination encourages research to better understand the mechanism of the synergy. OsMFCs are the precursor of such synergy, and thus understanding of OsMFCs will help to understand other 
integrations towards further development. It has been demonstrated that OsMFC could produce more electricity than an MFC containing cation exchange membrane (CEM) in both batch mode and continuous mode (Ge et al. 2013b, Zhang et al. 2011a). Electricity can be generated by using either sodium chloride or artificial seawater as both catholyte and draw solution (Zhang et al. 2011a), but sodium chloride resulted in the best performance of electricity generation and water flux among several tested draw solutes such as potassium phosphate buffer, $\mathrm{CaCl}_{2}$ and glucose (Ge and He 2012).

Although the use of FO membrane can lead to more electricity generation than that of CEM, it is not clear what factors contribute to this improvement. In general, the performance of an MFC is affected by its internal losses, including ohmic loss, activation loss, bacterial metabolic loss and concentration loss (Logan et al. 2006). Especially, replacing CEM with FO membrane could affect membrane resistance, variation of $\mathrm{pH}$ in catholyte and/or anolyte, and oxygen diffusion. It is also of strong interest to understand how water flux, a unique phenomenon for OsMFCs, can influence electricity generation. It was reported that an air-cathode OsMFC had a relatively lower internal resistance than the ones containing CEM or AEM according to the polarization curve, which might attribute to the acceleration of ion transport due to water flux (Werner et al. 2013). Proton movement could also be promoted by water flux, resulting in less increase in the catholyte $\mathrm{pH}$ and decrease in the anolyte $\mathrm{pH}$ (Zhang et al. 2011a). In an air-cathode OsMFC, the decreased anolyte $\mathrm{pH}(0.86 \mathrm{pH}$ unit $)$ was $24 \%$ of the theoretical value, whilst the increased catholyte $\mathrm{pH}(4.06 \mathrm{pH}$ unit) was only $63 \%$ of the theoretical value and $73 \%$ of that in a CEM-equipped MFC (Werner et al. 2013). Those findings indicate that the OsMFC may exert positive effect on $\mathrm{pH}$ stabilization 
and thus effectively reduce overpotential. In addition, the use of the high-conductivity catholyte can also help reduce the resistance of the catholyte, resulting in greater power production.

To better understand the OsMFC performance, experiments were conducted for comparing with a CEM-MFC and under different operating conditions, and a mathematical model has been developed. To our knowledge, this is the first mathematical model for OsMFCs. The objectives of this study are: (1) investigating the improvement of current generation with FO membrane; (2) validating the developed OsMFC model with experimental data; and (3) predicating the contribution of different factors of internal resistance to current generation with water flux.

\subsection{Materials and Methods}

\subsubsection{The OsMFC/ MFC Setup and Operation}

The same reactor was used as either an OsMFC or an MFC, with FO membrane or CEM installed between the anode compartment and the cathode compartment. Each compartment had a liquid volume of $360 \mathrm{~mL}$. Stainless steel mesh and carbon cloth were placed on both sides of the FO membrane as support materials. Two pretreated carbon brushes (Gordon BrushMfg. Co. Inc., Commerce, CA, USA) were inserted into the anode compartment as the anode electrodes. The cathode electrode was a piece of carbon cloth coated with Pt as the catalyst $\left(0.3 \mathrm{mg} \mathrm{cm}^{-2}\right)$. The cathode compartment was aerated with air. The electrodes were connected by copper wires to an external resistor of $10 \Omega$. Before use, the FO membrane (Hydration Technology Innovations, LLC, Albany, OR, USA) was soaked in deionized (DI) water for 30 min according to the manufacturer's instructions. The surface area of the FO membrane was about $98 \mathrm{~cm}^{2}$. The active layer of membrane was facing the anode (feeding solution). 
The OsMFC/MFC was operated at room temperature of $\sim 21^{\circ} \mathrm{C}$. The anode was inoculated with the anaerobic sludge from the Peppers Ferry Regional Wastewater Treatment Plant (Radford, VA, USA). The anode was fed with an acetate solution containing (per L of DI water): sodium acetate, $1 \mathrm{~g} ; \mathrm{NH}_{4} \mathrm{Cl}, 0.15 \mathrm{~g} ; \mathrm{NaCl}, 0.5 \mathrm{~g} ; \mathrm{MgSO}_{4}, 0.015 \mathrm{~g} ; \mathrm{CaCl}_{2}, 0.02 \mathrm{~g} ; \mathrm{NaHCO}_{3}, 0.1 \mathrm{~g} ; \mathrm{KH}_{2} \mathrm{PO}_{4}, 0.53 \mathrm{~g} ;$ $\mathrm{K}_{2} \mathrm{HPO}_{4}, 1.07 \mathrm{~g}$; and trace element, $1 \mathrm{~mL}$ (Angenent and Sung 2001). Two operation modes were applied in this study, batch mode and continuous mode. In the batch mode, a 500-mL glass bottle was connected to the anode compartment as an anolyte reservoir, while a 750 -mL glass bottle was used as a catholyte reservoir. The cathode compartment of the OsMFC was filled with DI water to allow FO membrane to rest for $2 \mathrm{~h}$ every $22 \mathrm{~h}$ during the batch operation. In the continuous mode, the anolyte and the catholyte had the same feeding rate of $1.3 \mathrm{~mL} \mathrm{~min}^{-1}$, resulting in a hydraulic retention time (HRT) of $4.5 \mathrm{~h}$ in each compartment, and were recirculated at $100 \mathrm{~mL} \mathrm{~min}{ }^{-1}$.

\subsubsection{Measurement and Analysis}

The cell voltage was recorded every 5 min by a digital multimeter (2700, Keithley Instruments Inc., Cleveland, $\mathrm{OH}$, USA). The $\mathrm{pH}$ was measured using a benchtop $\mathrm{pH}$ meter (Oakton Instruments, Vernon Hills, IL, USA). The conductivity was measured by a benchtop conductivity meter (Mettler-Toledo, Columbus, OH, USA). The polarization curve was performed by a potentiostat (Reference 600, Gamry Instruments, Warminster, PA, USA) at a scan rate of $0.2 \mathrm{mV}$

$\mathrm{s}^{-1}$. The volumetric densities of power and current were calculated based on the liquid volume of the anode compartment, according to a previous study (Zhang et al. 2010). Two Ag/AgCl reference electrodes (0.197 mV vs standard hydrogen electrode, SHE) were installed close to the anode electrode and cathode electrode, respectively. The anode potential and cathode potential were 
measured with the digital multimeter. The COD was measured using a DR/890 colorimeter (HACH Co., Ltd., USA) according to manufacturer's instruction. Water flux into the cathode (L $\mathrm{m}^{-2} \mathrm{~h}^{-1}, \mathrm{LMH}$ ) was calculated by the change of weight recorded on the balance (Scort Pro, Ohous, Columbia, MD, USA) (Wang et al. 2010a). The analysis of electrochemical impedance spectroscopy (EIS) of the membrane was conducted with different concentration of $\mathrm{NaCl}$ solution according to a previous research (Ge et al. 2013b). Coulombic efficiency (CE) was calculated as previously described (Logan et al. 2006).

\subsubsection{OsMFC Model Development}

\subsubsection{Mass balance of substrate in the anode.}

The OsMFC model was developed based on previously developed two-population MFC model, MDC model and FO model (Cath et al. 2006, Ping et al. 2014, Pinto et al. 2010) Anodophilic and methanogenic microbial populations are considered as the microbial community in the anode. The substrate concentration change is calculated based on the substrate consumption rates by two groups of microorganisms:

$$
\frac{d S}{d t}=\frac{Q_{\text {anode }, \text { in }}}{V_{\text {anode }}} S_{\text {in }}-\frac{Q_{\text {anode }, \text { out }}}{V_{\text {anode }}} S-k_{s, a, \max } \frac{S}{K_{a}+S} \frac{M_{O X}}{K_{M}+M_{O X}} C_{a}-k_{s, m, \max } \frac{S}{K_{m}+S} C_{m}
$$

(Equation 3.1)

where $S$ is the concentration of the substrate $\left(\mathrm{mg}-\mathrm{S} \cdot \mathrm{L}^{-1}\right) ; S_{i n}$ is the concentration of the influent substrate $\left(\mathrm{mg}-\mathrm{S} \cdot \mathrm{L}^{-1}\right) ; Q_{\text {anode, in }}$ and $Q_{\text {anode, out }}$ are the influent and effluent flow rates of the substrate $\left(\mathrm{L} \cdot\right.$ day $\left.^{-1}\right)$, respectively; $V_{\text {anode }}$ is the volume of the anode compartment $(\mathrm{L}) ; C_{a}$ and $C_{m}$ are the concentrations of anodophilic and methanogenic microorganisms $\left(\mathrm{mg}-\mathrm{C} \cdot \mathrm{L}^{-1}\right)$, respectively; $k_{s, a, m a x}$ and $k_{s, m, \max }$ are the maximum substrate consumption rates by anodophilic and methanogenic 
microorganisms (mg-S $\cdot \mathrm{mg}-\mathrm{a}^{-1} \cdot \mathrm{day}^{-1}$ and $\mathrm{mg}-\mathrm{S} \cdot \mathrm{mg}-\mathrm{m}^{-1} \cdot \mathrm{day}^{-1}$ ), respectively; Mox is the oxidized mediator fraction per anodophillic microorganism $\left(\mathrm{mg}-\mathrm{M} \cdot \mathrm{mg}-\mathrm{a}^{-1}\right)$; and, $K_{a}, K_{m}$, and $K_{M}$ are the half-saturation concentrations for the anodophillic microorganisms, methanogenic microorganisms, and the redox mediator $\left(\mathrm{mg}-\mathrm{S} \cdot \mathrm{L}^{-1}, \mathrm{mg}-\mathrm{S} \cdot \mathrm{L}^{-1}\right.$, and $\left.\mathrm{mg}-\mathrm{M} \cdot \mathrm{mg}-\mathrm{a}^{-1}\right)$, respectively.

The concentrations of two groups of microorganisms are represented in the following Monod kinetic differential equations:

$$
\begin{gathered}
\frac{d C_{a}}{d t}=\mathrm{k}_{a, \max } \frac{S}{K_{a}+S} \frac{M_{O X}}{K_{M}+M_{O X}} C_{a}-k_{d, a} C_{a}-\frac{1+\tanh \left(k_{a, x}\left(C_{a}+C_{m}-C_{a, \max }\right)\right)}{2} \frac{Q_{\text {anode,out }}}{V_{\text {anode }}} C_{a} \\
\text { (Equation 3.2) } \\
\frac{d C_{m}}{d t}=k_{\max \max } \frac{S}{K_{m}+S} C_{m}-k_{d, m} C_{m}-\frac{1+\tanh \left(k_{m, x}\left(C_{a}+C_{m}-C_{m, \text { max }}\right)\right)}{2} \frac{Q_{\text {anode,out }}}{V_{\text {anode }}} C_{m}
\end{gathered}
$$

(Equation 3.3)

where $k_{d, a}$ and $k_{d, m}$ are the decay rates of the microorganisms $\left(\right.$ day $\left.^{-1}\right) ; k_{a, m a x}$ and $k_{m, \max }$ are the maximum microorganism growth rates $\left(\right.$ day $\left.^{-1}\right) ; k_{\alpha, x}$ and $k_{m, x}$ are the steepness factors for anodophillic microorganism $\left(\mathrm{L} \cdot \mathrm{mg}-\mathrm{a}^{-1}\right)$ and methanogenic microorganisms $\left(\mathrm{L} \cdot \mathrm{mg}-\mathrm{m}^{-1}\right)$ for the biofilm retention; $C_{\alpha, \max }$ and $C_{m, \max }$ are the maximum attainable concentrations for anodophillic microorganism $\left(\mathrm{mg}-\mathrm{a} \cdot \mathrm{L}^{-1}\right)$ and methanogenic microorganisms $\left(\mathrm{mg}-\mathrm{m} \cdot \mathrm{L}^{-1}\right)$.

The current generation is calculated as:

$$
I_{M F C}=\frac{E_{O C}-O P_{\text {conc }}}{R_{\mathrm{int}}+R_{\mathrm{ext}}} \frac{M_{\text {red }}}{\varepsilon+M_{\mathrm{red}}}
$$

where $E_{O C}$ is the open circuit voltage (V); $O P_{\text {conc }}$ is the concentration overpotential (V); $M_{\text {red }}$ is the reduced mediator fraction per microorganisms $\left(\mathrm{mg}-\mathrm{M} \cdot \mathrm{mg}-\mathrm{a}^{-1}\right) ; \varepsilon$ is the constant $\left(\mathrm{mg}-\mathrm{M} \mathrm{mg}-\mathrm{x}^{-1}\right)$; $R_{\text {ext }}$ is the external resistance $(\Omega) ; R_{\text {int }}$ is the internal resistance of the cell $(\Omega)$ that is given in 
Equation 3 and made up of anode electrode resistance, cathode electrode resistance, electrolyte resistance, and membrane resistance (Fan et al. 2008).

$$
\begin{aligned}
& R_{\text {int }}=R_{\text {membrane }}+R_{\text {anolyte }}+R_{\text {catholyte }}+R_{\text {electrode }} \\
& R_{\text {anolyte }}=\frac{l}{C_{\text {anolyte }} \times A_{p}} \\
& R_{\text {catholyte }}=\frac{l}{C_{\text {catholyte }} \times A_{p}} \\
& R_{\text {membrane,CEM }}=\beta_{C E M}\left(\frac{1}{C_{\text {catholyte }}}+\frac{1}{C_{\text {anolyte }}}\right) \\
& R_{\text {membrane,FO }}=\beta_{F O}\left(\frac{1}{C_{\text {catholyte }}}+\frac{1}{C_{\text {anolyte }}}\right)
\end{aligned}
$$

where $R_{\text {membrane }}$ is the membrane resistance $(\Omega), R_{\text {electrode }}$ is the anode and cathode electrode resistance $(\Omega)$, and $R_{\text {anolyte }}$ and $R_{\text {catholyte }}$ are the resistance for anolyte and catholyte $(\Omega)$, respectively. For anolyte and catholyte, the resistance is inversely proportional to the conductivity of the solution, which can be calculated by Equation 3.6 and 3.7 (Galama et al. 2014, Sleutels et al. 2009). The $C_{\text {anolyte }}$ and $C_{\text {catholyte }}$ are the anolyte and catholyte solution conductivity $\left(\mathrm{S} \mathrm{m}^{-1}\right), l$ is the distance between the electrode and membrane $(\mathrm{m})$, and $A_{p}$ is the projected area for electrode $\left(\mathrm{m}^{2}\right)$. According to EIS, membrane resistance for the CEM and FO are also inversely proportional to the conductivity of the solution, which is consistent with the previous study (Sleutels et al. 2009). It was showed that the presence of a salt concentration difference across the membrane dramatically increased the membrane resistance compared to the value measured with EIS using the highconcentration solution in both sides (Galama et al. 2014, Geise et al. 2013). The $R$ membrane for CEM and FO membrane are calculated by Equation 3.8 and 3.9 with different coefficient $\beta_{C E M}$ and $\beta_{F O}$, respectively. 


\subsubsection{Mass balance of the salt in the OsMFC}

High concentration salt is used in OsMFC cathode side to extract clean water from wastewater. Besides, the salt could diffuse to the anode side due to the reverse salt flux. Ordinary differential equations shown below are developed to quantify the salt concentration:

$$
\begin{aligned}
& \frac{d C_{\text {salt }, a}}{d t}=\frac{Q_{\text {anode }, \text { in }}}{V_{\text {anode }}} C_{\text {salt }, a, \text { in }}-\frac{Q_{\text {anode }, \text { out }}}{V_{\text {anode }}} C_{\text {salt }, a}+\frac{J_{s}}{V_{\text {anode }}} \cdot A_{m} \\
& \frac{d C_{\text {salt }, c}}{d t}=\frac{Q_{\text {cathode }, \text { in }}}{V_{\text {cathode }}} C_{\text {salt }, \text {, in }}-\frac{Q_{\text {cathode }, \text { out }}}{V_{\text {cathode }}} C_{\text {salt }, c}-\frac{J_{s}}{V_{\text {anode }}} \cdot A_{m} \\
& Q_{\text {cathode,out }}=Q_{\text {cathode, }, \text { in }}+J_{w} \\
& Q_{\text {anode,out }}=Q_{\text {anode }, \text { in }}-J_{w}
\end{aligned}
$$

where $C_{s a l t, a}$ and $C_{s a l t, c}$ are the salt concentrations in the anode and cathode compartments (molsalt $\left.\cdot \mathrm{L}^{-1}\right)$, respectively; $C_{\text {salt,a,in }}$ and $C_{\text {salt, }, \text { in }}$ are the salt concentrations in the anode influent and cathode influent (mol-salt $\left.\cdot \mathrm{L}^{-1}\right)$, respectively; the reverse salt flux $J_{S}\left(\mathrm{~mol} \mathrm{~m}^{-2} \mathrm{~d}^{-1}\right)$ could be calculated by Equation 3.15; the water flux $J_{w}$ (LMH) could be calculated by Equation 3.14; $A_{m}$ is the membrane area $\left(0.0098 \mathrm{~m}^{2}\right)$.

\subsubsection{Water flux and reverse salt flux}

The water flux is simulated based on the FO mode (Lutchmiah et al. 2014a):

$$
J_{w}=A \cdot R T i\left(C_{s a l t, c}-C_{s a l t, a}\right)
$$

where $A$ is the permeability coefficient for FO membrane $\left(1.20 \times 10^{-7} \mathrm{~m}^{3} \mathrm{~m}^{-2} \mathrm{~s}^{-1} \mathrm{bar}^{-1}\right), i$ is the number of dissolved species ( 2 for $\mathrm{NaCl}), R\left(8.3145 \mathrm{~J} \mathrm{~mol}^{-1} \mathrm{~K}^{-1}\right)$ is the ideal gas constant, and $T$ $(294 \mathrm{~K})$ is the absolute temperature.

The reverse salt flux $J_{S c a n}$ be approximated as (Phillip et al. 2010): 
$J_{S}=\frac{B J_{w}}{A i R T}$

(Equation 3.15)

where $B$ is the salt permeability coefficient $\left(1.39 \times 10^{-7} \mathrm{~m}^{3} \mathrm{~m}^{-2} \mathrm{~s}^{-1}\right)$.

This OsMFC model can also be applied to MFCs, when both $J_{w}$ and $J_{s}$ are zero. The parameters were estimated as previously described and listed in Table 3.1, the relative root-mean square error was calculated to evaluate the discrepancy between the experimental data and the simulation results, and the mass balances for microorganisms in the anode were according to a previous study (Ping et al. 2014).

\section{Table 3.1 Parameters used in the OsMFC model}

\begin{tabular}{|c|c|c|c|}
\hline Parameter & Description & Value & Unit \\
\hline$Q_{a n o d e, \text { in }}$ & Flow rate of substrate feed to anode & 1.872 & $\mathrm{~L} \cdot \mathrm{day}^{-1}$ \\
\hline Qanode,out & Flow rate feeding to cathode & 1.872 & $L \cdot$ day $^{-1}$ \\
\hline $\boldsymbol{k}_{s, \boldsymbol{a}, \max }$ & $\begin{array}{l}\text { Maximum substrate consumption rate by anodophilic } \\
\text { microorganisms }\end{array}$ & 7.44 & $\begin{array}{l}\mathrm{mg}-\mathrm{S} \cdot \mathrm{mg}- \\
\mathrm{a}^{-1} \cdot \mathrm{day}^{-1}\end{array}$ \\
\hline $\boldsymbol{k}_{s, \boldsymbol{m}, \max }$ & $\begin{array}{l}\text { Maximum substrate consumption rate by } \\
\text { methanogenic microorganisms }\end{array}$ & 2.55 & $\begin{array}{l}\mathrm{mg-S} \cdot \mathrm{mg}- \\
\mathrm{m}^{-1} \cdot \text { day }^{-1}\end{array}$ \\
\hline Mox & $\begin{array}{l}\text { Oxidized mediator fraction per anodophillic } \\
\text { microorganism }\end{array}$ & 0.005 & $\mathrm{mg}-\mathrm{M} \cdot \mathrm{mg}-\mathrm{a}^{-1}$ \\
\hline$K_{a}$ & $\begin{array}{l}\text { Half-saturation concentrations for the anodophillic } \\
\text { microorganisms }\end{array}$ & 20 & $\mathrm{mg}-\mathrm{S} \cdot \mathrm{L}^{-1}$ \\
\hline$K_{m}$ & $\begin{array}{l}\text { Half-saturation concentrations for the methanogenic } \\
\text { microorganisms }\end{array}$ & 80 & $\mathrm{mg}-\mathrm{S} \cdot \mathrm{L}^{-1}$ \\
\hline$K_{M}$ & Half-saturation concentrations for the redox mediator & 0.01 & $\mathrm{mg}-\mathrm{M} \cdot \mathrm{mg}-\mathrm{a}^{-1}$ \\
\hline$M_{\text {red }}$ & Reduced mediator fraction per microorganisms & 0.0428 & $\mathrm{mg}-\mathrm{M} \cdot \mathrm{mg}-\mathrm{a}^{-1}$ \\
\hline$\varepsilon$ & Constant & 0.0001 & $\mathrm{mg}-\mathrm{M} \mathrm{mg}-\mathrm{x}^{-1}$ \\
\hline $\boldsymbol{R}_{\text {membrane }}$ & Membrane resistance & 7 & $\Omega$ \\
\hline$A_{p}$ & Projected area for electrode & 0.0098 & $\mathrm{~m}^{2}$ \\
\hline $\boldsymbol{A}$ & Permeability coefficient for FO membrane & $1.20 \times 10^{-7}$ & $\mathrm{~m}^{3} \mathrm{~m}^{-2} \mathrm{~s}^{-1}$ bar $^{-1}$ \\
\hline $\boldsymbol{B}$ & Salt permeability coefficient for FO membrane & $1.39 \times 10^{-7}$ & $\mathrm{~m}^{3} \mathrm{~m}^{-2} \mathrm{~s}^{-1}$ \\
\hline $\boldsymbol{R}$ & Ideal gas constant & 8.3145 & $\mathrm{~J} \mathrm{~mol}^{-1} \mathrm{~K}^{-1}$ \\
\hline$T$ & Temperature & 294 & $\mathrm{~K}$ \\
\hline
\end{tabular}




\subsection{Results and Discussion}

\subsubsection{Comparing OsMFC with MFC}

Although the previous study has reported the comparison between OsMFCs and MFCs, that comparison was preliminary and the results were limited by the low electricity generation due to inefficient reactor configuration (Zhang et al. 2011a). In this study, a more detailed comparison was conducted under both open and closed circuit conditions with measuring individual potentials. Operated in a batch mode and with $35 \mathrm{~g} \mathrm{~L}^{-1} \mathrm{NaCl}$ solution as the catholyte, the OCV of the OsMFC was $0.76 \mathrm{~V}$, comparable to that of the conventional MFC (Figure 3.1A). After 12-h operation, the OCV of the OsMFC began to drop rapidly, while the OCV of the MFC did not start to decrease until $19 \mathrm{~h}$. The cathode potentials for both the OsMFC and the MFC were stable during the batch operation. However, the anode potential varied over time and caused the different OCV for the two MFCs. Since the $\mathrm{pH}$ of the anode effluent was not apparently decreased in both the OsMFC and the MFC ( $7.15 \pm 0.11)$, the drop of anode potential was not due to the $\mathrm{pH}$. With water flux in the OsMFC, the volume of the anolyte decreased from $750 \mathrm{~mL}$ to $287 \pm 29 \mathrm{~mL}$ during the whole batch, which could concentrate the substrate and background salts in the anolyte compared to that of the MFC. When the batch ended, the catholyte $\mathrm{pH}$ for OsMFC was $8.56 \pm 0.12$ while the catholyte $\mathrm{pH}$ for MFC was $10.18 \pm 0.17$. The difference in catholyte $\mathrm{pH}$ increase was probably caused by water flux and faster transport of protons across the FO membrane (Werner et al. 2013, Zhang et al. 2011a): the water extracted from the anolyte diluted the catholyte in terms of the concentrations of both the salt and the accumulated $\mathrm{OH}^{-}$; water flux might have promoted the transport of protons from the anolyte into the catholyte, thereby resulting in a lower $\mathrm{pH}$ of the catholyte; finally, reverse salt flux, a unique feature of FO membrane, could cause the movement 
of $\mathrm{OH}^{-}$from the catholyte into the anolyte, driven by the concentration gradient of $\mathrm{OH}^{-}$, leading to the difference in catholyte $\mathrm{pH}$ increase between the OsMFC and the MFC.

To generate current, a 10- $\Omega$ resistor was connected the circuit. As shown in Figure 3.1B, the current density of the OsMFC was much higher than that of the MFC. In the OsMFC, the COD concentration decreased from 785 to $210 \mathrm{mg} \mathrm{L}^{-1}$; because the volume of the anolyte also decreased from 750 to $251 \mathrm{~mL}$ due to water extraction (Figure 3.1C), the COD removal efficiency in the OsMFC was about $91 \%$. The COD concentration in the MFC anode effluent was $81 \mathrm{mg} \mathrm{L}^{-1}$, representing a COD removal efficiency of $90 \%$. The Coulombic efficiency (CE) of the OsMFC (32\%) was higher than that obtained in the MFC (18\%), consistent with the previous findings (Werner et al. 2013, Zhang et al. 2011a). The cathode potential in the MFC is slightly higher than that in the OsMFC, probably related to the lower ionic strength of the OsMFC catholyte due to the extracted water diluting the salt concentration during the operation. In general, the similar potential of the anode/cathode electrodes between the OsMFC and the MFC did not explain the difference in their current generation. One hypothesis is that solution and membrane resistance, is a key factor to the improvement of current generation in the OsMFC.

After 13-h operation, difference between the cathode potential and the anode potential was negative, indicating that voltage reversal might have happened. Previous studies indicate that this voltage reversal was due to the substrate depletion in the anolyte, resulting in loss of bacterial activity (An and Lee 2014, Oh and Logan 2007). However, in a single MFC, the anode potential and cathode potential should converge together with no reversal voltage under the situation of fuel starvation. The "voltage reversal" in this MFC/OsMFC, which could be a pseudo-phenomenon, 
was likely related to deviation caused by the measurement of electrode potential. When the substrate supply was sufficient, the solution resistance between the anode/cathode electrode and reference electrode could be neglected compared to the large resistance of the digital multimeter. However, when the substrate was depleted, the solution resistance increased to an extent that it cannot be neglected; that led to the increase of the measured anode potential (from negative to
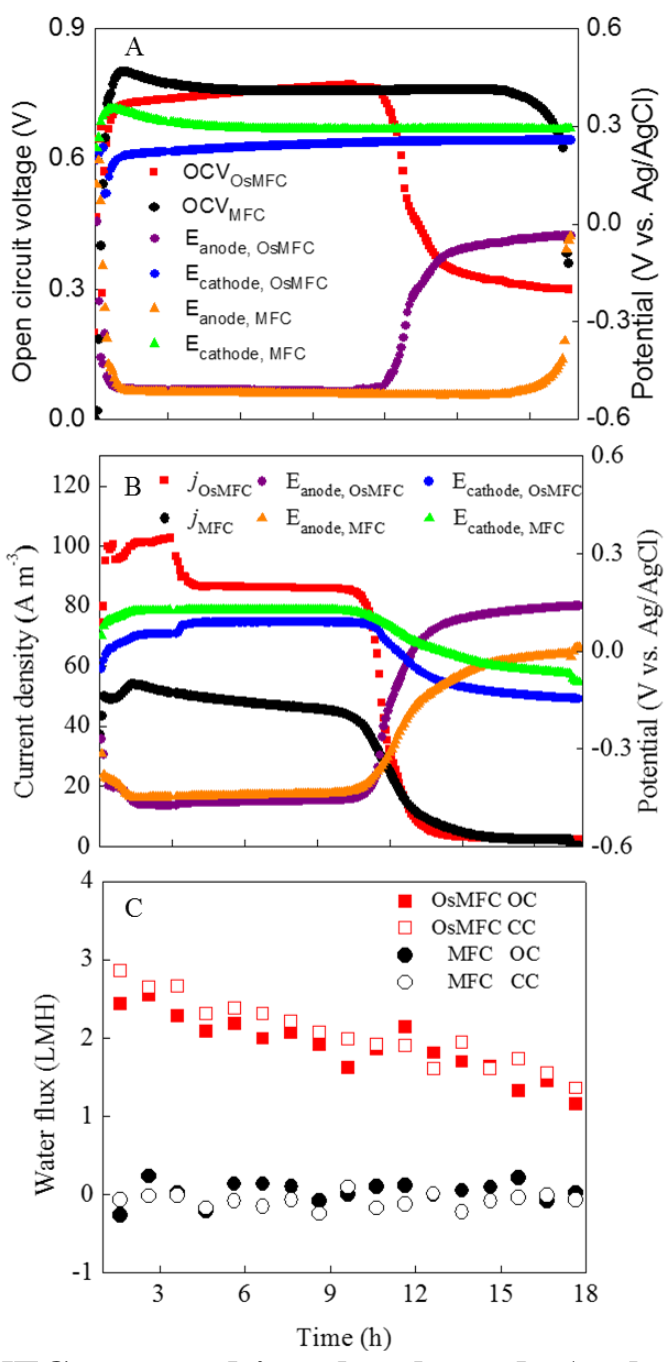

Figure 3.1 The OsMFC and MFC operated in a batch mode (catholyte: $35 \mathrm{~g} \mathrm{~L}^{-1} \mathrm{NaCl}$ ): (A) open circuit voltage and the individual potentials, $(B)$ current density $(j)$ and the anode and cathode potential under a closed circuit condiction, and (C) water flux in open circuit (OC) and closed circuit (CC). 
zero) and decrease in the cathode potential (from positive to zero) after 13-h operation as the solution resistance increased. Therefore, it is likely that the actual voltage did not reverse.

\subsubsection{Effects of membrane and solution resistance}

The effects of membrane and solution resistance were investigated in two steps: first, the conductivity of the anolyte and the catholyte was kept similar so that there was no water flux; and second, the conductivity of the catholyte was adjusted by using combined $\mathrm{NaCl}$ and glucose while keeping the similar osmotic pressure. In the first step, $2 \mathrm{~g} \mathrm{~L}^{-1} \mathrm{NaCl}$ solution was used as a catholyte to prevent water flux in both the OsMFC and the MFC (Figure 3.2A). As shown in Figure 2B, the OsMFC generated a higher current density of $75.2 \mathrm{~A} \mathrm{~m}^{-3}$ than the MFC $\left(42.8 \mathrm{~A} \mathrm{~m}^{-3}\right)$, while both achieved similar COD removal efficiency of $\sim 85 \%$. Consequently, the CE of the OsMFC was $30 \%$, higher than $14 \%$ obtained in the MFC. With similar potential of the anode/cathode electrodes (Figure 3.2C) and similar anolyte/catholyte conductivity, the increased current generation in the OsMFC was likely related to the difference in membrane resistance, which was $9.7 \Omega$ for FO membrane in the OsMFC and $14.3 \Omega$ for CEM in the MFC.

In the second step, different combinations of sodium chloride and glucose were used to supply same driving force (osmotic pressure) for water flux but different electrical conductivity (Table 3.2). Glucose has a very low conductivity but could generate osmotic pressure as a draw solute in FO processes (Xie et al. 2012). Figure 3.3A shows that the electrical conductivity of the catholyte clearly affects the current generation. The catholyte containing no glucose (S-5) had a conductivity of $55.6 \mathrm{mS} \mathrm{cm}^{-1}$, resulting in a current density of $118.5 \pm 2.6 \mathrm{~A} \mathrm{~m}^{-3}$, significantly higher than 13.4 $\pm 3.4 \mathrm{~A} \mathrm{~m}^{-3}$ obtained with the catholyte containing no $\mathrm{NaCl}(\mathrm{S}-1)$ that had a conductivity of 0.084 $\mathrm{mS} \mathrm{cm} \mathrm{cm}^{-1}$. The total coulomb generated during one batch cycle was strongly correlated to the 
catholyte conductivity, with a $\mathrm{R}^{2}$ of 0.9781 (inset, Figure $3.3 \mathrm{~A}$ ). The water flux was slightly affected by the different combination of sodium chloride and glucose (Figure 3.3B).
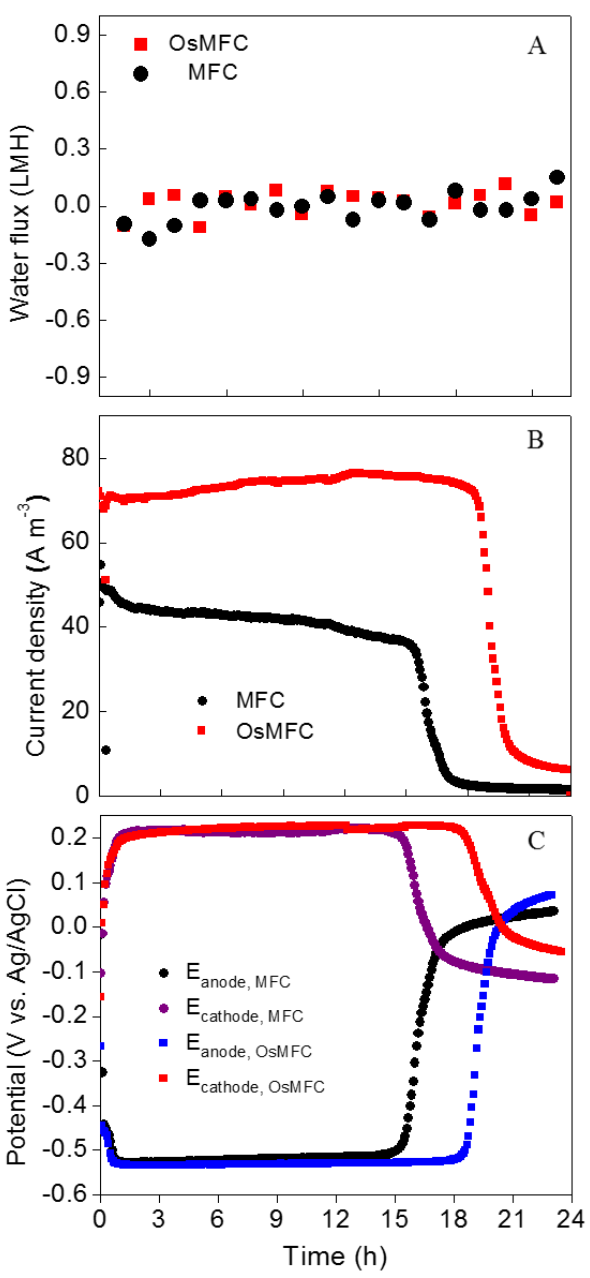

Figure 3.2 The OsMFC and the MFC operated with no water flux (catholyte: $2 \mathrm{~g} \mathrm{~L}^{-1}$ $\mathrm{NaCl}$ ): (A) water flux, (B) current density, and (C) individual potentials. 
Table 3.2 The characterization of $\mathrm{NaCl}$ and glucose catholyte

\begin{tabular}{|c|c|c|c|c|c|c|c|}
\hline & $\begin{array}{l}\mathrm{NaCl} \\
\left(\mathrm{g} \mathrm{L}^{-1}\right)\end{array}$ & $\begin{array}{l}\text { Glucose } \\
\left(\mathbf{g ~ L ^ { - 1 }}\right)\end{array}$ & $\begin{array}{l}\text { Influent } \\
\text { conductivity } \\
\left(\mathrm{mS} \mathrm{cm}^{-1}\right)\end{array}$ & $\begin{array}{l}\text { Effluent } \\
\text { conductivity } \\
\left(\mathbf{m S ~ c m} \mathbf{~ c m}^{-1}\right)\end{array}$ & $\begin{array}{l}\text { Effluent } \\
\text { COD } \\
\left(\mathrm{mg} \mathrm{L}^{-1}\right)\end{array}$ & $\begin{array}{l}\text { The } \\
\text { total } \\
\text { coulomb } \\
\text { (C) }\end{array}$ & $\mathrm{CE}$ \\
\hline S-1 & 0 & 214 & 0.084 & 0.37 & 213 & 384.96 & $7.44 \%$ \\
\hline S-2 & 10 & 154 & 13.08 & 8.99 & 181 & 651.14 & $11.92 \%$ \\
\hline S-3 & 20 & 92 & 27.80 & 18.53 & 299 & 807.19 & $18.36 \%$ \\
\hline$S-4$ & 30 & 31 & 44.40 & 30.10 & 205 & 1006.26 & $19.18 \%$ \\
\hline S-5 & 35 & 0 & 55.60 & 35.70 & 217 & 1737.88 & $33.83 \%$ \\
\hline
\end{tabular}

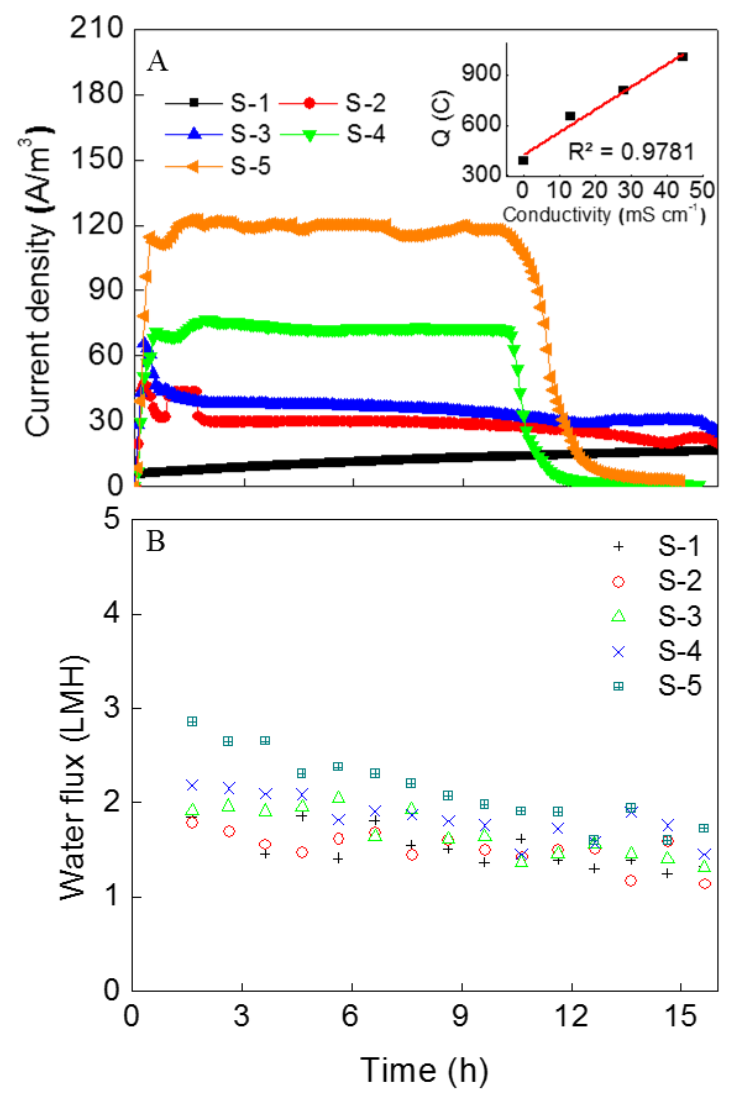

Figure 3.3 The OsMFC with different catholyte conductivity: (A) current density, and (B) water flux. S1-5 are explained in Table 2. 


\subsubsection{OsMFC model fitting and validation}

Mathematical modeling can help understand the key factors in the proposed system and may explain some parameters that can hardly studied with experiments. Herein, the first OsMFC model has been developed based on the information obtained from a continuously operated OsMFC. The established model was validated with two different catholyte concentrations $\left(35 \mathrm{~g} \mathrm{~L}^{-1}\right.$ and $2 \mathrm{~g} \mathrm{~L}^{-1}$ $\mathrm{NaCl})$. In general, the model predication of current generation, salt concentration, and water flux shows satisfactory agreement with the experimental measurement, in which the current decreased from 27 to $17 \mathrm{~mA}$ and water flux dropped from 2.25 to nearly zero LMH when the catholyte influent concentration was reduced from 35 to $2 \mathrm{~g} \mathrm{~L}^{-1}$ (Figure 3.4A, C and D). However, some overestimation/underestimation can be seen from the modeled variation trend of the COD concentration as shown in Figure 3.4B. The predicted effluent COD increased when the $\mathrm{NaCl}$ concentration decreased to $2 \mathrm{~g} \mathrm{~L}^{-1}$. But the COD was not significantly influenced by the catholyte change in the experiment. The overestimation of the COD removal was mainly due to the current generation drop. In the model, it was assumed that anodophillic microorganism was the dominant community, but in reality it might not happen ideally, thereby resulting in the over/underestimation of the COD removal at lower current. Furthermore, this model could also fit the MFC when there is no water flux and the FO membrane resistance replaced by the CEM membrane resistance (date not shown).

The OsMFC model was validated through varying the concentration of $\mathrm{NaCl}$ in the catholyte from 2 to $10 \mathrm{~g} \mathrm{~L}^{-1}$ (Figure 3.5). In response to the change, the current increased from 18 to $23 \mathrm{~mA}$, with the water flux increased from 0 to $0.7 \mathrm{LMH}$. The relative RMSE for the current was $8.06 \%$, while the RMSE for the water flux was $31.95 \%$ due to the unstable water flux in the first few points. For 
the salt concentrations in the anode and the cathode, the relative RMSE were $3.19 \%$ and $7.17 \%$, respectively. Those results suggest that the OsMFC model could give a good fit for the key parameters of the OsMFC.
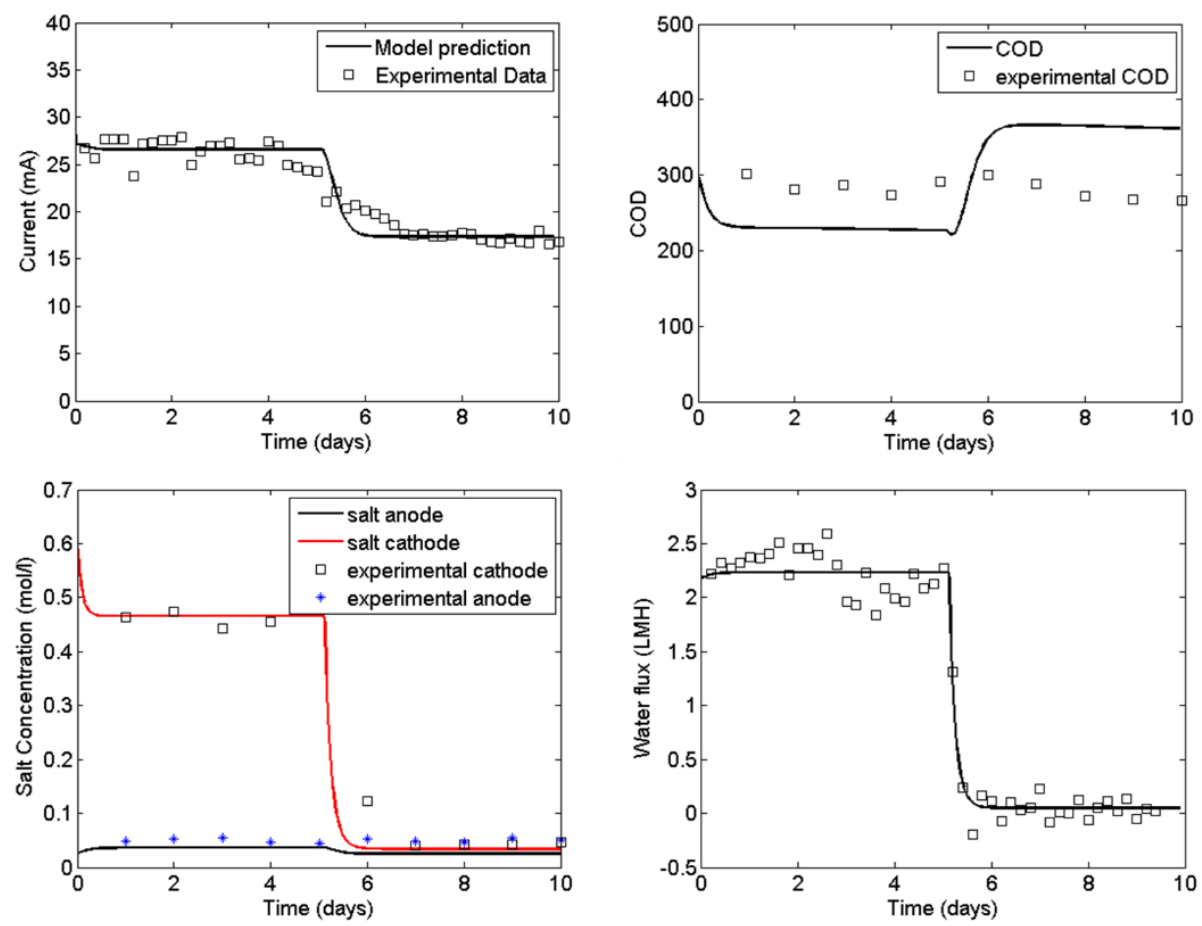

Figure 3.4 Model fitting for the OsMFC when the catholyte was changed from 35 to $2 \mathrm{~g} \mathrm{~L}^{-1}$ NaCl: (A) current generation, (B) COD concentration in anode effluent, (C) salt concentrations in the anolyte and the catholyte, and (D) water flux. 

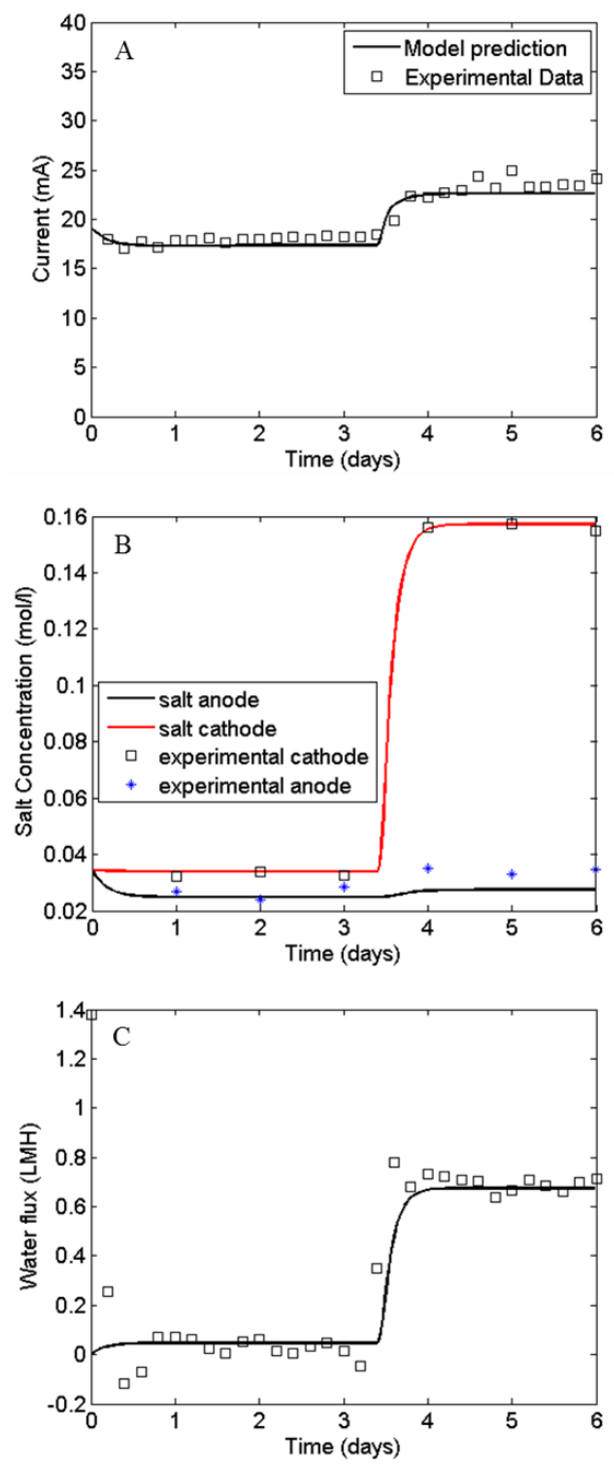

Figure 3.5 The OsMFC model validation when the catholyte was changed from $2 \mathrm{~g}$ $\mathrm{L}^{-1} \mathrm{NaCl}$ to $10 \mathrm{~g} \mathrm{~L}^{-1} \mathrm{NaCl}$ solution: (A) current generation, (B) salt concentrations of anolyte and catholyte, and $(C)$ water flux.

\subsubsection{OsMFC model prediction}

The validated OsMFC model was used to predict the distribution of internal resistance (anode electrode resistance, cathode electrode resistance, electrolyte resistance, and membrane resistance) in the OsMFC. For comparison, the predication was also conducted for the MFC. In this model, the electrode resistance is set as a constant $(7 \Omega)$ according to the polarization curve and negligible 
change in the anode and the cathode potentials. As shown in Equations 6-9, the electrolyte (solution) resistance and membrane resistance are related to the solution conductivity. The salt concentration in the cathode is varied to predict the variation of electrolyte resistance and membrane resistance to explain why the OsMFC could generate more electricity than the MFC. As shown in Figure 3.6A, the current generation increases as the cathode salt concentration increased for both the OsMFC and the MFC. In the OsMFC, the current density increases from 38.3 to $73.9 \mathrm{~A} \mathrm{~m}^{-3}$ as the cathode $\mathrm{NaCl}$ concentration increases from 1 to $35 \mathrm{~g} \mathrm{~L}^{-1}$, while the current of the MFC increases from 35.2 to $59.6 \mathrm{~A} \mathrm{~m}^{-3}$.

The distribution of the internal resistance of the OsMFC and the MFC varies as the cathode salt concentration changes (Figure 3.6B and C). When the cathode $\mathrm{NaCl}$ concentration increases from 1 to $35 \mathrm{~g} \mathrm{~L}^{-1}$, the internal resistance decreases from 45.33 to $18.46 \Omega$ in the OsMFC and 50.33 to $25.47 \Omega$ in the MFC. The difference in the internal resistance is most likely resulted from the difference in both electrolyte resistance and membrane resistance. In the MFC, the conductivity of the anolyte remains the same with an anolyte resistance of $8.49 \Omega$. In the OsMFC, the conductivity of anolyte increases as the cathode salt concentration increases due to concentrating effect with the increased water flux permeates through the FO membrane. That results in the decrease in the anolyte resistance from 8.53 to $5.74 \Omega$ as the cathode $\mathrm{NaCl}$ concentration increases from 1 to $35 \mathrm{~g}$ $\mathrm{L}^{-1}$. The catholyte resistance of the OsMFC decreases from 12.17 to $0.45 \Omega$; however, it is still higher than that of the MFC at the same cathode salt concentration, because of the dilution with the permeated water. Due to the high salt concentration in the catholyte, the difference in the catholyte resistance between the two systems has minor contribution to the difference in electricity generation. 

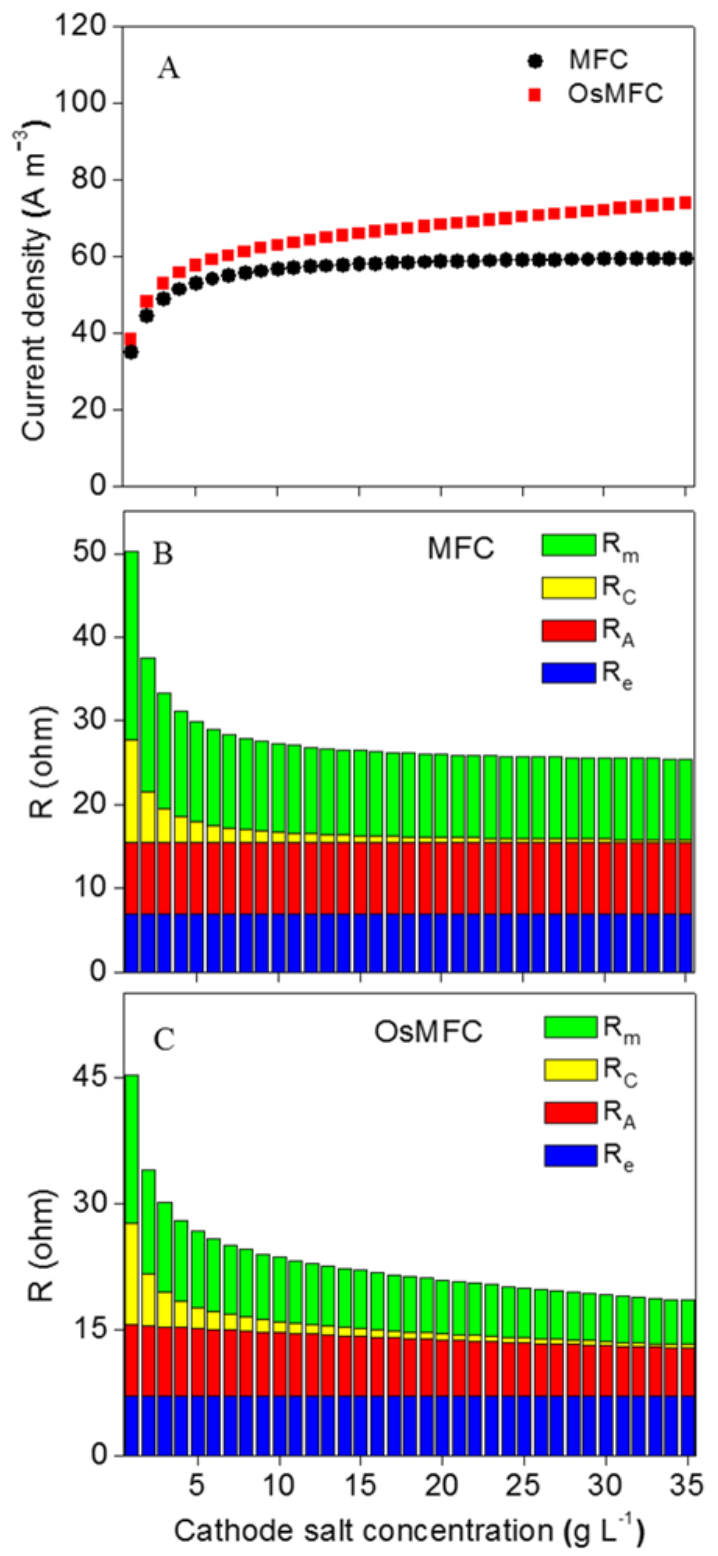

Figure 3.6 Model prediction for the OsMFC and the MFC with varied salt concentration in the catholyte: (A) current generation in OsMFC and MFC, (B) internal resistance distribution in MFC, and (C) internal resistance distribution in OsMFC. $R_{\mathrm{m}}$ : membrane resistance; $R_{C}$ : catholyte resistance; $R_{\mathrm{A}}$ : anolyte resistance; $\mathbf{R}_{\mathrm{e}}$ : electrode resistance.

The membrane properties such as membrane thickness, molar conductivity and mobility of the charge carriers could influence the membrane resistance (Leong et al. 2013, Wang et al. 2010c). The varied catholyte and anolyte conductivity with the water flux in the OsMFC can affect its 
membrane resistance. As the cathode $\mathrm{NaCl}$ concentration increases from 1 to $35 \mathrm{~g} \mathrm{~L}^{-1}$, the membrane resistance of FO decreases from 17.62 to $5.27 \Omega$, while the CEM resistance decreases from 22.60 to $9.63 \Omega$. In the OsMFC, the contribution of membrane resistance to the internal resistance decreases with the increased catholyte salt concentration, while in the MFC the membrane resistance has relatively stable contribution $(>37 \%)$ to the overall resistance.

\subsection{Conclusions}

This study has confirmed that OsMFCs can generate more electricity than regular MFCs, and provided initial explanation that the enhancement is mainly due to lower membrane resistance, which is affected by both membrane materials and water flux. The developed mathematical model for OsMFCs could well fit the experimental data, and effectively predict the variation in the internal resistance with water flux. The integrated experimental investigation and mathematical model have advanced the understanding of electricity generation in OsMFCs, and revealed the key factors for further development of this technology towards simultaneous wastewater treatment, bioenergy recovery, and water reclamation. 


\title{
Chapter 4 Effects of Current Generation and Electrolyte pH on Reverse Salt Flux across Thin Film Composite Membrane in Osmotic Microbial Fuel Cells
}

(This section has been published as Qin, M., Abu-Reesh, I. M., \& He, Z.* (2016). Effects of Current Generation and Electrolyte $\mathrm{pH}$ on Reverse Salt Flux across Thin Film Composite Membrane in Osmotic Microbial Fuel Cells. Water research, 105, 583-590.)

\begin{abstract}
Osmotic microbial fuel cells (OsMFCs) take advantages of synergy between forward osmosis (FO) and microbial fuel cells (MFCs) to accomplish wastewater treatment, current generation, and highquality water extraction. As an FO based technology, OsMFCs also encounter reverse salt flux (RSF) that is the backward transport of salt ions across the FO membrane into the treated wastewater. This RSF can reduce water flux, contaminate the treated wastewater, and increase the operational expense, and thus must be properly addressed before any possible applications. In this study, we aimed to understand the effects of current generation and electrolyte $\mathrm{pH}$ on RSF in an OsMFC. It was found that electricity generation could greatly inhibit RSF, which decreased from $16.3 \pm 2.8$ to $3.9 \pm 0.7 \mathrm{gMH}$ when the total Coulomb production increased from 0 to $311 \mathrm{C}$. The OsMFC exhibited $45.9 \pm 28.4 \%$ lower RSF at the catholyte $\mathrm{pH}$ of 3 than that at $\mathrm{pH} 11$ when $40 \Omega$ external resistance was connected. The amount of sodium ions transported across the FO membrane was $18.3-40.7 \%$ more than that of chloride ions. Ion transport was accomplished via diffusion and electrically-driven migration, and the theoretical analysis showed that the inhibited electrically-driven migration should be responsible for the reduced RSF. These findings are potentially important to control and reduce RSF in OsMFCs or other osmotic-driven processes.
\end{abstract}




\subsection{Introduction}

Osmotic microbial fuel cells (OsMFCs) are a new type of microbial fuel cells (MFCs) integrated with forward osmosis (FO) (Zhang et al. 2011a). By inheriting the functions of both MFCs and FO, an OsMFC can simultaneously generate electricity and extract water with the aid of a semipermeable FO membrane that rejects solute ions (Cath et al. 2006, Lutchmiah et al. 2014b), separates the anode and cathode, and extracts treated water from the anolyte (feed solution) into the catholyte (draw solution) (Lu et al. 2014b, Werner et al. 2013, Yuan and He 2015). Like regular MFCs, organic compounds in wastewater are oxidized at the anode of OsMFCs by exoelectrogenic microorganisms and the electrons generated from the anode oxidation reaction are transferred to the cathode through an external circuit where oxygen is reduced to water (oxygen reduction reaction) (Zhang et al. 2011a). In the past few years, OsMFCs have been studied and developed in the aspects such as substrates and operation optimization. OsMFCs can treat both synthetic solution and actual wastewater, and it was reported that more than $50 \%$ of the treated wastewater could be extracted from the anode (Ge et al. 2013b, Ismail and Ibrahim 2015). Operation optimization focused on selecting the optimal draw solute, determining membrane orientation, and simplifying the cathode. Draw solution (catholyte) plays a key role in the OsMFC operation, and a draw solute that has both a high conductivity and strong buffer capacity will benefit electricity generation and water flux (Ge and $\mathrm{He}$ 2012). The OsMFC having cellulose triacetate (CTA) embedded support exhibited the higher electricity generation due to the less permeability of oxygen than other membranes (Yang et al. 2015a, Yang et al. 2016b). Air cathodes have been developed to replace liquid cathodes for improving electricity generation in OsMFCs (Werner et al. 2013). In addition, fouling of FO membrane was found to benefit the current generation of an OsMFC despite the reduction of water flux (Zhu et al. 2016). The concept of OsMFCs has also 
been extended to create osmotic microbial electrolysis cells and osmotic microbial desalination cells (Lee et al. 2015, Zhang and He 2012).

As a technology based on the principle of FO, the OsMFCs would inherit some intrinsic drawbacks of FO, for example, reverse salt flux (RSF) can hardly be avoided and is one of the most challenging issues (Phillip et al. 2010). RSF occurs due to a concentration gradient across an FO membrane, resulting in backward transport of draw solutes into the feed side. Such reverse transport reduces the driving force for water transport, increases the operation cost due to loss of draw solutes, and contaminates the feed solution (Cath et al. 2006). RSF can also result in salt accumulation within the fouling layer on the membrane surface, which increases the cakeenhanced osmotic pressure and therefore, exhibits a negative impact on the process efficiency (Boo et al. 2012). In an FO process, an ideal FO membrane should have high water permeability and low solute permeability, to achieve high water flux and low RSF (Cath et al. 2006); however, high water flux usually leads to high RSF (Lutchmiah et al. 2014b). For example, the commercial thinfilm composite (TFC) FO membrane, which is comprised of an ultra-thin polyamide active layer and a porous polysulfone support layer, is typically more water permeable and has relatively higher RSF compared to conventional asymmetric cellulose acetate (CA) membrane. In an OsMFC, although RSF can decrease the solution resistance and thus benefit the anode activity, overly accumulated salts will negatively influence the microbial activity and impair the quality of the treated effluent, which has been demonstrated in osmotic membrane bioreactors that had decreased organic removal efficiency with accumulated salts due to RSF (Alturki et al. 2012, Lu and He 2015). Therefore, it is vitally important to reduce RSF for sustainable implementation of FO-based water and wastewater treatment technologies. 
A unique feature of OsMFCs, which makes it different from other FO-based technologies, is electricity generation. Compared to conventional MFCs, OsMFCs can generate more electricity under both a batch mode and a continuous mode using either sodium chloride solution or artificial seawater as the catholyte (Ge et al. 2013b, Zhang et al. 2011a). Such improved performance of electricity generation was attributed to the lower internal resistance of the OsMFCs than that of the MFCs (Werner et al. 2013). An OsMFC mathematical model was developed, and subsequently predicted a reduction of internal resistance with increasing osmotic pressure gradient and water flux, thereby confirming the importance of membrane resistance (Qin et al. 2015). The lower membrane resistance in OsMFCs is related to both a lower $\mathrm{pH}$ gradient across the membrane due to the improved proton flux promoted by water flux and higher anolyte conductivity in the presence of reverse salt flux (Zhu et al. 2015). Movement of electrons as a result of bioelectrochemical reactions can affect ion transport across ion exchange membranes (Chen et al. 2012, Chen et al. 2016, Ping et al. 2016). For example, it was reported that the current generation in microbial desalination cells (MDCs) could inhibit back diffusion of ions from the anolyte into the desalinated stream (Ping et al. 2016). In addition, the electric field has been employed to reduce the membrane fouling in membrane bioreactors via the electrostatic repulsion between negative charged foulants and the cathode (Bani-Melhem and Elektorowicz 2010, Chen et al. 2007, Liu et al. 2013). Thus, we hypothesized that the current generated in OsMFCs could inhibit RSF. A key factor that potentially correlates with both electricity generation and RSF is the electrolyte $\mathrm{pH}$. It has been reported that RSF can vary significantly as a function of solution $\mathrm{pH}$ and increase at alkaline $\mathrm{pH}$ in the FO process (Arena et al. 2015). The carboxylic acid groups in TFC membrane could gain or lose protons depending on the solution $\mathrm{pH}$, leading to a varied cation transport across 
the membrane (Holloway et al. 2015); however, it is not clear how this process will be affected by electricity generation.

In this study, we have examined the above hypothesis and aimed to understand the effects of current generation and electrolyte $\mathrm{pH}$ on RSF across TFC membranes in an OsMFC. Batch OsMFC experiments were conducted with varied current generation and/or electrolyte $\mathrm{pH}$. The specific objectives were to: (1) examine whether RSF could be reduced by current generation; (2) investigate how electrolyte $\mathrm{pH}$ would affect $\mathrm{RSF}$ through interaction with current generation; and (3) reveal the mechanism of RSF and relevant ion transport in the OsMFC.

\subsection{Materials and Methods}

\subsubsection{OsMFC setup}

The schematic of the OsMFC is shown in Figure 4.1 and the experimental setup is shown in Figure 4,2. The OsMFC consisted of two equal-size compartments $\left(14 \times 7 \times 3.7 \mathrm{~cm}^{3} /\right.$ each with a liquid volume of $280 \mathrm{~mL}$ ) as the anode and the cathode, respectively, separated by a TFC FO membrane (Hydration Technology Innovations, LLC, Albany, OR, USA) with its active layer facing the anode (feed) side. Plastic mesh was placed on both sides of the FO membrane as support materials. Two carbon brushes (Gordon Brush Mfg. Co. Inc., Commerce, CA, USA) were pretreated by being soaked in pure acetone overnight and heat-treated in a muffle furnace (Model 550 Isotemp Series, Fisher Scientific, Pittsburgh, PA, USA) at $450{ }^{\circ} \mathrm{C}$ for 30 minutes; after pretreatment they were inserted into the anode compartment as the anode electrodes. The cathode electrode was a piece of carbon cloth coated with Platinum $(\mathrm{Pt})$ as the catalyst $\left(0.3 \mathrm{mg} \mathrm{Pt} \mathrm{cm}^{-2}\right)$. Before use, the TFC membrane was soaked in deionized (DI) water for $30 \mathrm{~min}$ according to the manufacturer's instruction (Stillman et al. 2014). The surface area of the FO membrane was about $98 \mathrm{~cm}^{2}$. A 500- 
$\mathrm{mL}$ bottle was connected to the anode compartment as a reservoir and placed on a digital balance for monitoring water flux. A 300-mL glass bottle containing the catholyte was connected to the cathode as a reservoir. The cathode compartment was aerated with the air to provide oxygen for cathode reaction and create flow turbulence.

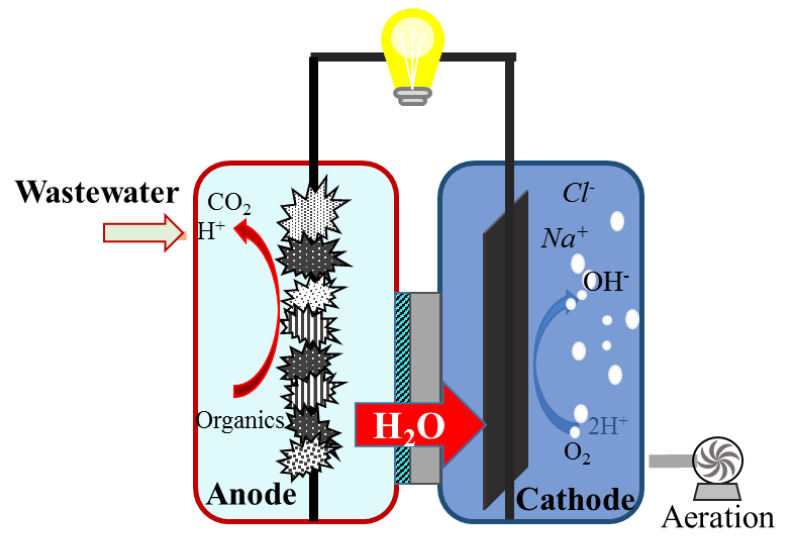

TFC

Figure 4.1 Schematic of an osmotic microbial fuel cell.

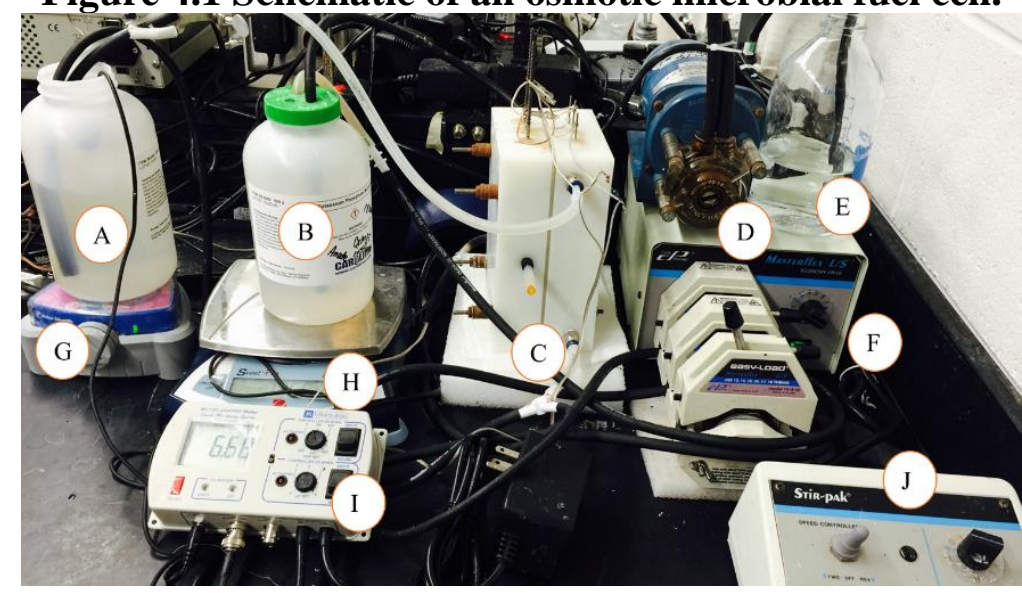

Figure 4.2 Photograph of OsMFC. A, anolyte reservoir; B, catholyte reservoir; C, OsMFC reactor; $\mathrm{D}$, pump for $\mathrm{pH}$ adjustment; $\mathrm{E}, 1 \mathrm{M} \mathrm{NaOH} / \mathrm{HCl}$ solution; $\mathrm{F}$, recirculation pump; $\mathrm{G}$, stir plate; H, mass balance; I, pH meter; J, pump speed controller, and $\mathrm{K}$, aeration pump (for catholyte).

\subsubsection{OsMFC operation}

The OsMFC was operated at room temperature of $\sim 21^{\circ} \mathrm{C}$. The anode compartment was inoculated with the anaerobic sludge from the Peppers Ferry Regional Wastewater Treatment Plant (Radford, VA, USA). The anode feeding solution contained (per L of DI water): sodium acetate, $0.5 \mathrm{~g}$; 
$\mathrm{NH}_{4} \mathrm{Cl}, 0.075 \mathrm{~g} ; \mathrm{NaCl}, 0.25 \mathrm{~g} ; \mathrm{MgSO}_{4}, 0.0075 \mathrm{~g} ; \mathrm{CaCl}_{2}, 0.01 \mathrm{~g} ; \mathrm{NaHCO}_{3}, 0.05 \mathrm{~g} ; \mathrm{KH}_{2} \mathrm{PO}_{4}, 0.27 \mathrm{~g}$; $\mathrm{K}_{2} \mathrm{HPO}_{4}, 0.53 \mathrm{~g}$; and trace element, $0.5 \mathrm{~mL}$ (Angenent and Sung 2001). The cathode was fed with $35 \mathrm{~g} \mathrm{~L}^{-1} \mathrm{NaCl}$ solution. The OsMFC was operated with $500 \mathrm{~mL}$ anolyte and $300 \mathrm{~mL}$ catholyte in a batch mode (6 hours). After 6-h operation, the data was collected and all the solutions (in both compartments and reservoirs) were replaced. During night, the OsMFC was operated with 1-L anolyte and 500-mL $5 \mathrm{~g} \mathrm{~L}^{-1} \mathrm{NaCl}$ solution without data collection for 12 hours. The same OsMFC reactor was used for all the tests, to avoid the difference in performance caused by reactor manufacturing. To study the effects of current generation, the external resistance was manipulated to five levels ( $1 \Omega, 10 \Omega, 40 \Omega, 100 \Omega$ and quasi-infinite (open circuit mode)), and the catholyte $\mathrm{pH}$ was fixed at 7 with $1 \mathrm{M} \mathrm{HCl}$ solution. In the experiments of controlled catholyte $\mathrm{pH}$, the $\mathrm{pH}$ of the draw solution (catholyte) was maintained at 3, 7 or 11 with $1 \mathrm{M} \mathrm{HCl}$ or $\mathrm{NaOH}$. In the study of uncontrolled catholyte $\mathrm{pH}$, two experiments were performed: First, the catholyte $\mathrm{pH}$ was initially adjusted to 3, 7 and 11 with $1 \mathrm{M} \mathrm{HCl}$ or $\mathrm{NaOH}$ and $40 \Omega$ external resistance was connected to the OsMFC; second, the external resistance was manipulated to four levels ( $1 \Omega, 10 \Omega, 40 \Omega$ and 100 $\Omega$ ) for different current generation, and the catholyte $\mathrm{pH}$ was initially 7 without any further adjustment during the 6-h experiment. The initial $\mathrm{pH}$ was adjusted and measured before each experiment. Both the anolyte and catholyte were recirculated at $100 \mathrm{~mL} \mathrm{~min}^{-1}$. The FO membrane was washed with DI water for removing foulants every 2 days to reduce the effect of membrane fouling on the tests. The integrity tests for the FO membrane with Milli-Q water as the feed solution and 1- $\mathrm{M} \mathrm{NaCl}$ as the draw solution were performed after each wash. As shown in Figure 4.3, either the water flux or RSF was not significantly changed, suggesting that the FO membrane was relatively stable during the experiment and membrane fouling was reversible. Water 
permeability coefficients $(\mathrm{A})$ and solute $(\mathrm{NaCl})$ permeability coefficients $(\mathrm{B})$ were determined through the FO tests (Table 4.1).

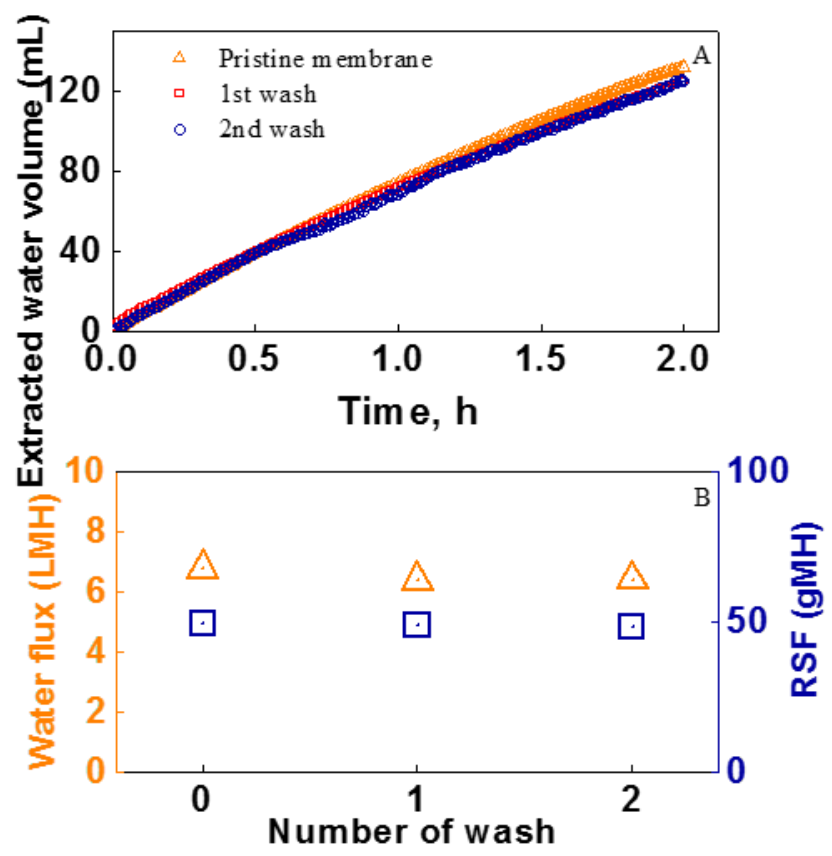

Figure 4.3 Integrity test for the FO membrane. (A) the extracted water volume during the 2-h operation for the pristine FO membrane and membrane after-wash; $(B)$ the water flux and RSF for the pristine FO membrane and membrane after-wash.

Table 4.1 TFC membrane properties.

\begin{tabular}{ccc}
\hline & Unit & TFC membrane \\
\hline Pure water permeability (A) & $\mathrm{L} / \mathrm{m}^{2}-\mathrm{h}-$ bar & 0.26 \\
Salt permeability (B) & $\mathrm{m} / \mathrm{s}$ & $4.55 \times 10^{-7}$ \\
\hline
\end{tabular}

\subsubsection{Measurement and analysis}

The OsMFC voltage was recorded every 2 minutes by a digital multimeter (2700, Keithley Instruments Inc., Cleveland, OH, USA). The polarization curve was performed by a potentiostat (Reference 600, Gamry Instruments, Warminster, PA, USA) at a scan rate of $0.2 \mathrm{mV} \mathrm{s}^{-1}$. The $\mathrm{pH}$ 
was measured using a benchtop $\mathrm{pH}$ meter (Oakton Instruments, Vernon Hills, IL, USA). The conductivity was measured by a benchtop conductivity meter (Mettler-Toledo, Columbus, OH, USA). The volumetric densities of power and current were calculated based on the liquid volume of the anode compartment, according to a previous study (Zhang et al. 2010). The concentration of chemical oxygen demand (COD) was measured using a DR/890 colorimeter (HACH Co., Ltd., USA) according to the manufacturer's instruction. The catholyte weight in the reservoir was recorded every 1 min on a balance (Scort Pro, Ohous, Columbia, MD, USA) (Wang et al. 2010b). The concentrations of $\mathrm{Na}^{+}$and $\mathrm{Cl}^{-}$were quantified by using ion chromatography (Dionex LC20 ion chromatograph, Sunnyvale, CA, U.S.A.) equipped with an ED40 eletrochemical detector. The total Coulomb generated in OsMFC was calculated as:

$$
\text { Total } \operatorname{Coulomb}(\mathrm{C})=\sum I(A) t(s)
$$

where $I$ is electric current and $t$ is the time.

Water flux $J_{w}$ across the FO membrane $\left(\mathrm{L} \mathrm{m}^{-2} \mathrm{~h}^{-1}, \mathrm{LMH}\right)$ was calculated by the change of catholyte solution weight over time (Wang et al. 2010b).

$$
J_{w}=\frac{V_{c, t}-V_{c, 0}}{A_{m} t}
$$
where $V_{c, t}$ and $V_{c, 0}$ are the volume (L) of the catholyte at the test time $t(\mathrm{~h})$ and 0 , respectively. The difference of $V_{c, t}$ and $V_{c, o}$ equals to the weight increase of the draw solution in value (kg) during this period; $A_{m}$ is the membrane area $\left(\mathrm{m}^{2}\right)$.

$\operatorname{RSF}\left(J_{s}, \mathrm{~g} \mathrm{~m}^{-2} \mathrm{~h}^{-1}, \mathrm{gMH}\right)$ from the cathode (draw) to the anode (feed) was calculated based on the salt concentration change in the feed solution (Wang et al. 2010b).

$$
J_{s}=\frac{c_{a, t} V_{a, t}-c_{a, 0} V_{a, 0}}{A_{m} t}
$$


where $C_{a, t}$ and $C_{a, 0}$ are the ion concentrations in the feed solution at time t $(6 \mathrm{~h})$ and 0 , respectively; $V_{a, t}$ and $V_{a, 0}$ are the volume (L) of the anolyte at time $\mathrm{t}(6 \mathrm{~h})$ and 0 , respectively.

The pure water permeability coefficient $\left(\mathrm{A}, \mathrm{L} \mathrm{m}^{-2}\right.$-h-bar) and salt permeability coefficient $\left(\mathrm{B}, \mathrm{m} \mathrm{s}^{-}\right.$ $\left.{ }^{1}\right)$ were calculated as:

$J_{w}=A\left(\pi_{D}-\pi_{F}\right)$

(Equation 4.4)

where $\pi_{D}$ and $\pi_{F}$ are the osmotic pressure of the draw solution at the membrane interface and osmotic pressure of the feed solution at the feed-membrane interface, respectively (Cath et al. 2013)

and

$\frac{J_{W}}{J_{S}}=\frac{A}{B} n R_{g} T$

(Equation 4.5) where $n$ is the number of species that the draw solute dissociates into (e.g., $n=2$ for $\mathrm{NaCl}$ ), $R_{g}$ is the gas constant, and $T$ is the absolute temperature.

The RSF includes diffusion and electrically-driven migration (EDM) according to the NernstPlanck equation:

$J_{S}=J_{D}+J_{M}$

(Equation 4.6)

where $J_{D}$ is the flux due to diffusion and $J_{M}$ is the flux due to EDM.

Diffusion $\left(J_{D}\right)$ can be calculated by (Phillip et al. 2010):

$J_{D, i}=-\frac{D_{i}}{t_{M}}\left(C_{d, i}-C_{f, i}\right)$

(Equation 4.7)

where $\mathrm{Di}$ is the diffusion coefficient of species $\mathrm{i}, \mathrm{tM}$ is the thickness of TFC membrane (m), Cd,i is the concentration of species $i$ on the catholyte, and Cf,i is the concentration of species $i$ in the anolyte. The diffusion coefficient is calculated as in a previous study (Table 4.2) (Kim et al. 2007a). 
Table 4.2 Diffusion coefficient in TFC membrane.

\begin{tabular}{cccc}
\hline Ion & & Unit & TFC membrane \\
\hline & $\mathrm{Na}^{+}$ & $\mathrm{m}^{2} / \mathrm{s}$ & $5.38 \times 10^{-9}$ \\
& $\mathrm{Cl}^{-}$ & $\mathrm{m}^{2} / \mathrm{s}$ & $5.26 \times 10^{-9}$ \\
\hline
\end{tabular}

The percentage of RSF contributed by Diffusion $\left(J_{D}\right)$ and EDM ( $J_{M}$ were calculated by the Equations 4.8 and 4.9 , respectively.

$$
\begin{aligned}
& J_{D} \%=\frac{J_{D}}{J_{S}} \\
& J_{M} \%=1-\frac{J_{D}}{J_{S}}
\end{aligned}
$$

(Equation 4.9)

\subsection{Results and Discussion}

\subsubsection{Current generation decreases RSF}

The influence of current generation on RSF was investigated by varying external resistance loading at a fixed catholyte $\mathrm{pH}$ of 7 . When the external resistance decreased from $100 \Omega$ to $1 \Omega$, the maximum current density of the OsMFC increased from $16 \mathrm{~A} \mathrm{~m}^{-3}$ to $94 \mathrm{~A} \mathrm{~m}^{-3}$ (Figure 4.4) while the total Coulomb production increased from $90 \mathrm{C}$ to $311 \mathrm{C}$. During the 6-h operation, the COD concentration in the anode decreased from $411 \pm 24 \mathrm{mg} \mathrm{L}^{-1}$ to $109 \pm 39 \mathrm{mg} \mathrm{L}^{-1}$ (mean value \pm standard deviation), and the anolyte $\mathrm{pH}$ remained relatively stable $(7.26 \pm 0.17)$ due to the buffer capacity of phosphate salts. In an open circuit mode, the effluent COD was $207 \pm 6 \mathrm{mg} \mathrm{L}^{-1}$ while the anolyte $\mathrm{pH}$ was $7.10 \pm 0.06$. The polarization test indicated that there was no obvious power overshoot in the high current zone where low external resistance was applied (Figure 4.5); thus, the current generation increased as the external resistance decreased. RSF clearly decreased with increasing current generation (Figure 4.4B). In the open circuit mode where there was no current generation and zero total Coulomb, RSF was $16.3 \pm 2.8 \mathrm{gMH}$. Applying an external resistor of 100 
$\Omega$ to the electrical circuit produced the total Coulomb of $90 \mathrm{C}$, and decreased RSF to $9.1 \pm 2.1$ gMH. Further decreasing the external resistance increased current generation and also reduced RSF. The lowest external resistance of $1 \Omega$ resulted in a total Coulomb production of $311 \mathrm{C}$ in a batch; meanwhile, RSF was $3.9 \pm 0.7 \mathrm{gMH}$, a reduction of $76.1 \pm 4.3 \%$ compared to that of the open circuit condition.

Water flux was not changed significantly with current/Coulomb production (Figure 4.4B and Figure 4.6), likely due to small change in osmotic pressure difference that resulted in little water flux variation. Water flux is governed by the osmotic pressure difference $(\Delta \pi)$ between the anode (feed) and the cathode (draw), and RSF can reduce $\Delta \pi$ by increasing the anolyte salinity and reducing the catholyte salinity. Dilution of the catholyte due to water extraction would also decrease $\Delta \pi$. After 6-h operation, the osmotic pressure difference in the OsMFC with $3.9 \pm 0.7$ gMH RSF was $86.3 \pm 0.8 \%$ of its initial $\Delta \pi$, while it was $78.5 \pm 0.8 \%$ of the initial $\Delta \pi$ in the open circuit mode with $16.3 \pm 2.8 \mathrm{gMH}$ RSF. The difference in final $\Delta \pi$ between those two conditions resulted from the different RSF, however, the final $\Delta \pi$ difference only contributed to $3.2 \pm 0.7 \mathrm{~mL}$ 
water extraction difference, which could be neglected during experimental measurement. Hence, current/Coulomb production did not significantly affect the water flux in OsMFCs.
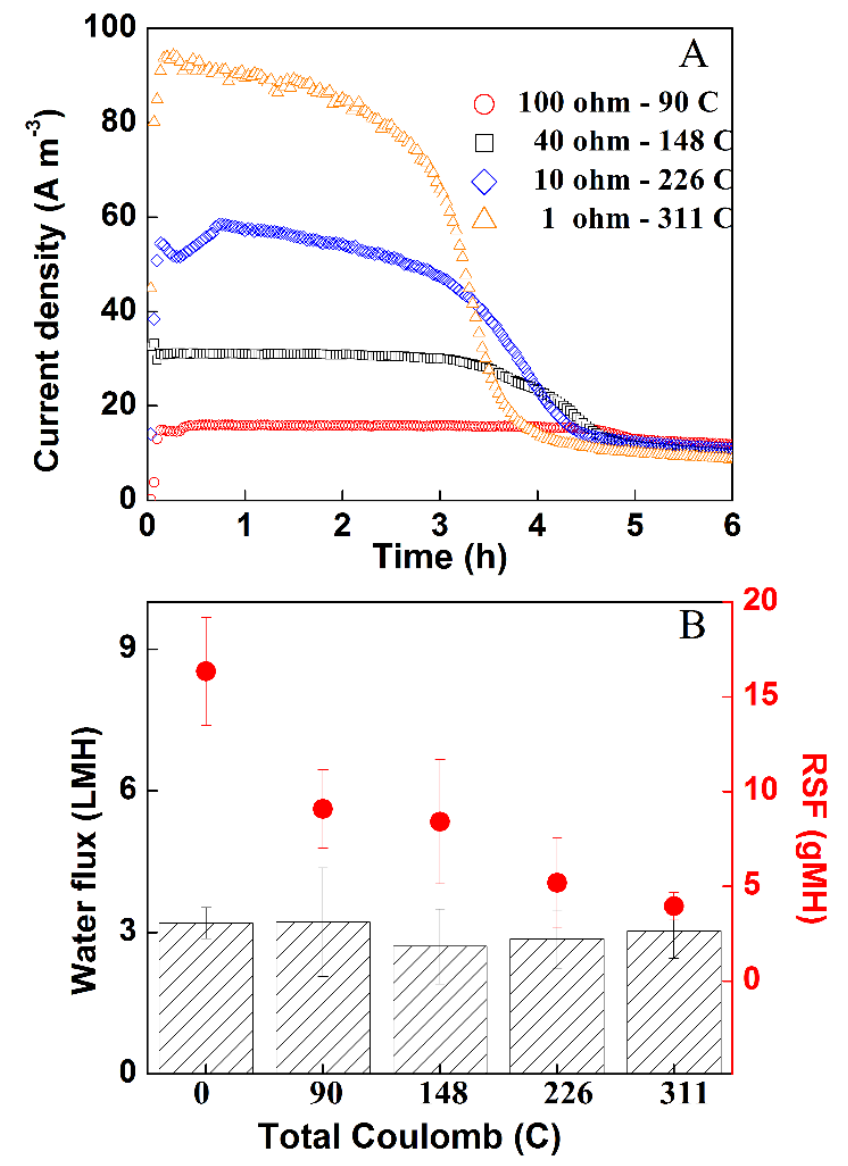

Figure 4.4 The OsMFC performance with different external resistance: (A) current generation; and (B) water flux and reverse salt flux (RSF).

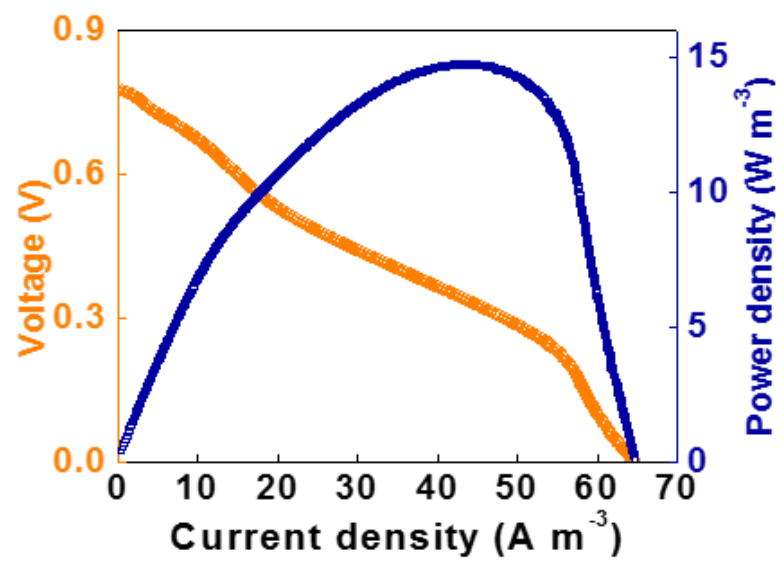

Figure 4.5 Polarization curve of the OsMFC. 

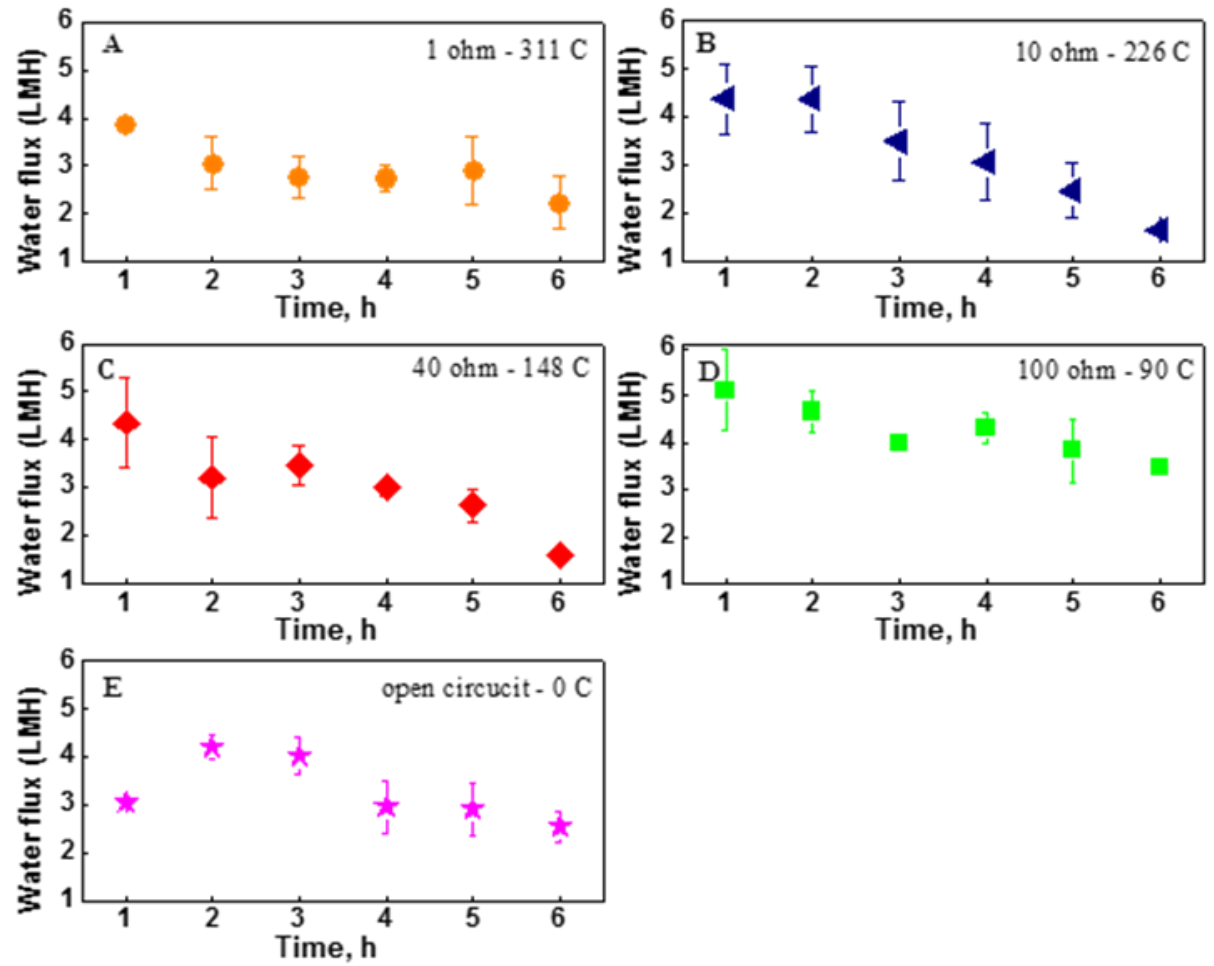

Figure 4.6 Water flux in OsMFC with different external resistance (Coulomb production): (A) $1 \Omega$ - 311 Coulomb (C) generation (B) $10 \Omega-226 \mathrm{C}$ generation (C) $40 \Omega$ - $148 \mathrm{C}$ generation (D) $100 \Omega$ - $90 \mathrm{C}$ generation, and (E) open circuit.

\subsubsection{Effects of controlled catholyte $\mathrm{pH}$}

It has been reported that the solution $\mathrm{pH}$ can significantly affect RSF in the FO system (Arena et al. 2015). The influence of solution $\mathrm{pH}$ on RSF in the OsMFC was investigated with the catholyte $\mathrm{pH}$ fixed at 3, 7 and 11, and with external resistance of 10,40, $100 \Omega$, and quasi-infinite (open circuit mode), respectively. The anolyte $\mathrm{pH}$ was buffered with phosphate buffer solution (initial $\mathrm{pH}: 7.20 \pm 0.04)$. After the 6 -h experiment, the anolyte $\mathrm{pH}$ slightly varied to $6.96 \pm 0.12,7.18 \pm$ 0.09 and $7.24 \pm 0.24$ along with a catholyte $\mathrm{pH}$ of 3,7 and 11 , respectively (Figure 4.7 ). The diffusion of $\mathrm{H}^{+}$or $\mathrm{OH}^{-}$from the catholyte to the anolyte might have affected the anolyte $\mathrm{pH}$. 


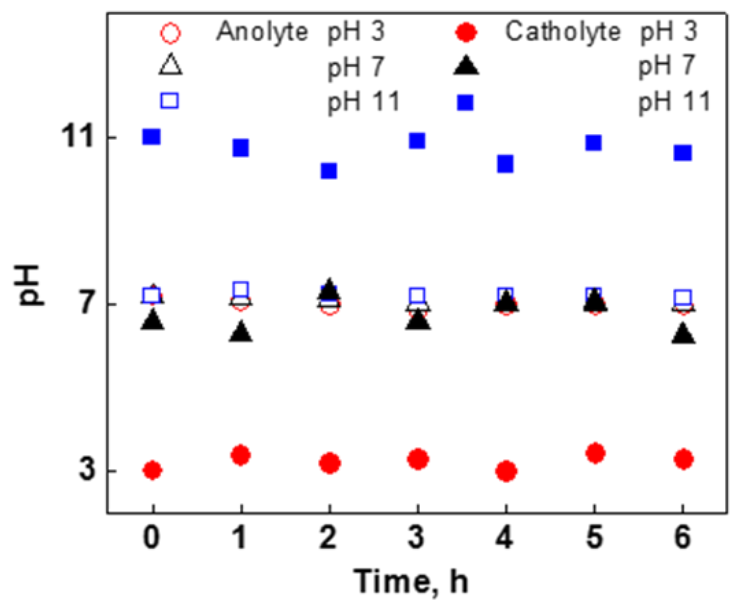

\section{Figure 4.7 pH variation in anolyte and catholyte with fixed catholyte pH. The} external resistance was $40 \Omega$.

The catholyte $\mathrm{pH}$ exhibited strong effects on RSF through interaction with current generation.

With the same external resistance, a higher catholyte $\mathrm{pH}$ led to a higher RSF (Figure 4.8A). For example, when the external resistance was $40 \Omega$, RSF was $6.6 \pm 2.2 \mathrm{gMH}, 8.4 \pm 3.3 \mathrm{gMH}$, and $12.2 \pm 1.2 \mathrm{gMH}$ at the catholyte $\mathrm{pH}$ of 3,7 and 11 , respectively. This difference was related to electricity generation under different $\mathrm{pH}$ conditions. The total Coulomb production with $40 \Omega$ was $171 \mathrm{C}, 153 \mathrm{C}$ and $142 \mathrm{C}$ at the catholyte $\mathrm{pH}$ of 3,7 and 11 , respectively (Figure 4.8B). When operated in the open circuit mode (without current generation), the OsMFC showed the highest RSF under every $\mathrm{pH}$ (Figure 4.8A). A higher catholyte $\mathrm{pH}$ would create more potential loss (0.059 $\mathrm{V} / \mathrm{pH}$ ), resulting in lower electricity generation (Zhao et al. 2006). The lower electricity generation would have less inhibition of RSF. In addition, the high $\mathrm{pH}$ could affect the membrane surface chemistry, resulting in a higher RSF in an FO process (Arena et al. 2015). The reduction on RSF benefited from current generation was more significant at high catholyte $\mathrm{pH}$. For example, at the catholyte $\mathrm{pH}$ of $11, \mathrm{RSF}$ decreased from $18.1 \pm 0.7 \mathrm{gMH}$ to $7.0 \pm 2.0 \mathrm{gMH}$ when the external resistance decreased from $100 \Omega$ to $10 \Omega$ (the Coulomb production increased from 78 to $241 \mathrm{C}$, Figure 4.8B). This represents $57.0 \pm 3.1 \%$ reduction in $\mathrm{RSF}$, much higher than $27.8 \pm 4.3 \%$ (at $\mathrm{pH}$ 
3) and $42.9 \pm 2.5 \%$ (at $\mathrm{pH} 7$ ). Therefore, high current generation could depress the effect of high catholyte $\mathrm{pH}$ on RSF.
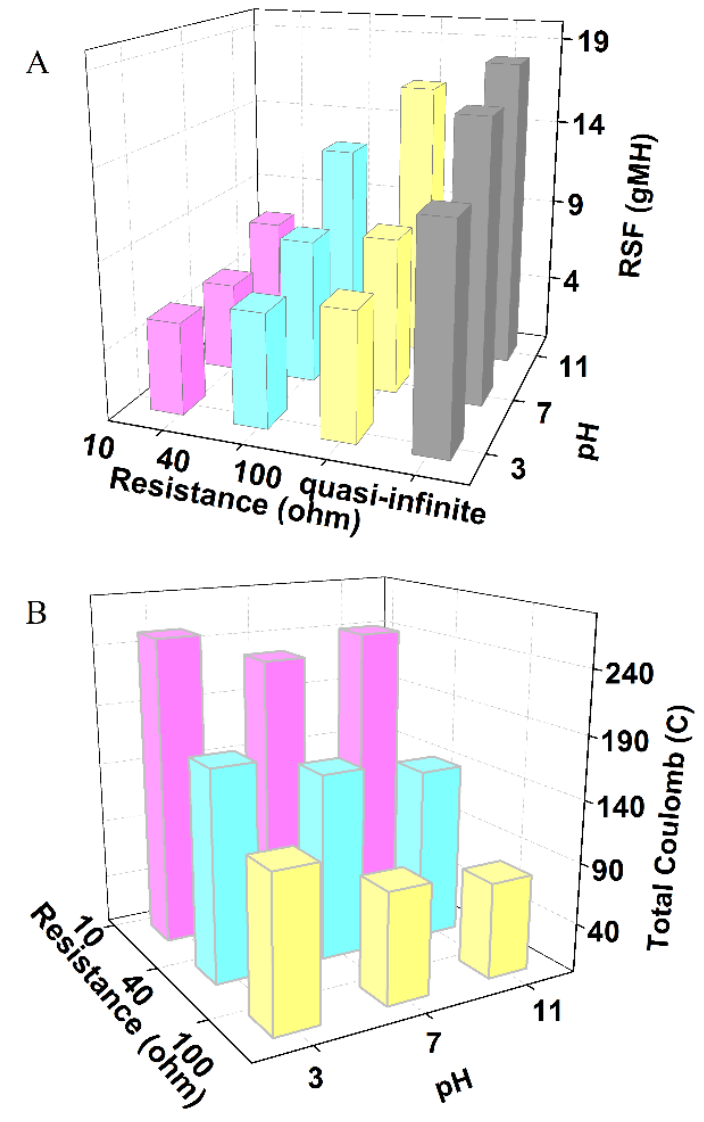

Figure 4.8 The effects of the controlled catholyte $\mathrm{pH}$ and external resistance: (A) RSF; and (B) total Coulomb production. Quasi-infinite resistance was in open circuit mode and didn't have current generation.

The effect of the anolyte $\mathrm{pH}$ on RSF was not investigated here but may occur. In the anode, the oxidation of the substrates would decrease the anode $\mathrm{pH}$ due to the generation of proton and lack of buffer capacity. When the anolyte was acidic, the amount of deprotonated carboxylic acid groups would decrease, which may decrease the RSF. However, a lower anolyte pH could inhibit the bioelectrocatalytic activity of anodic microorganisms, increase the internal resistance, and decrease the system stability (Harnisch and Schröder 2009, Raghavulu et al. 2009). As a result, 
current generation would decrease and have less inhibition on RSF, thereby resulting in a higher RSF.

\subsubsection{Uncontrolled catholyte $\mathrm{pH}$}

In actual operation of an OsMFC, maintaining a fixed catholyte $\mathrm{pH}$ would be very difficult because that the draw solutes do not always have a buffer capacity. Thus, we examined RSF and current generation with uncontrolled catholyte $\mathrm{pH}$. Two experiments were performed: the first was to understand the effect of $\mathrm{pH}$ variation on $\mathrm{RSF}$ at a fixed external resistor and the second test was to investigate the influence of current generation on RSF at initial catholyte $\mathrm{pH}$ of 7.

The influence of $\mathrm{pH}$ variation on RSF was investigated at external resistance of $40 \Omega$ with three initial catholyte $\mathrm{pH}: 3,7$ and 11 . The solution $\mathrm{pH}$ was not adjusted during the 6-h experiment. The RSF was $8.1 \pm 1.8 \mathrm{gMH}, 8.0 \pm 1.9 \mathrm{gMH}$, and $8.1 \pm 1.4 \mathrm{gMH}$ at the initial catholyte $\mathrm{pH}$ of 3,7 and 11, respectively (Figure 4.9A), indicating that the RSF was not affected by the initial catholyte $\mathrm{pH}$. These RSF data were consistent with the RSF in the OsMFC at the fixed catholyte $\mathrm{pH} 7$ and same external resistance $(8.4 \pm 3.3 \mathrm{gMH})$. In addition, the catholyte $\mathrm{pH}$ changed rapidly and became similar at the end of the testing period. Within the first hour of operation, the catholyte $\mathrm{pH}$ increased to 8.45 and 8.89 with the initial $\mathrm{pH}$ of 3 and 7 , respectively, or decreased to 10.22 from the initial $\mathrm{pH}$ of 11 (Figure 4.10A). At the end of the 6-h operation, the catholyte $\mathrm{pH}$ became 8.39, 8.41 and 9.19, with the initial catholyte $\mathrm{pH}$ of 3, 7 and 11, respectively. Meanwhile, the current generation was not significantly affected by the initial catholyte $\mathrm{pH}$ (Figure 4.9B). Therefore, the similar catholyte $\mathrm{pH}$ after the 6-h operation resulted in the similar RSF regardless of the initial catholyte $\mathrm{pH}$. The variation of catholyte $\mathrm{pH}$ could be related to the dilution effect of the catholyte, oxygen reduction reaction, and proton/hydroxide transport across the membrane. The dilution 

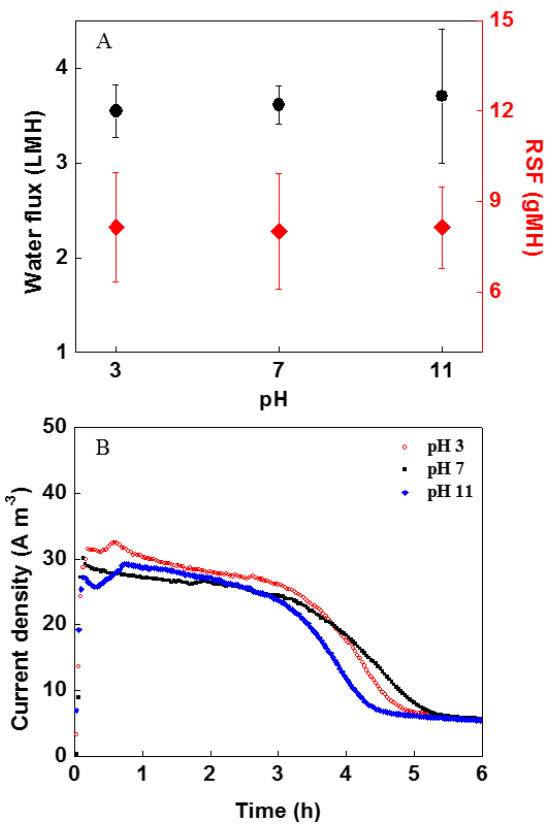

Figure 4.9 Performance of the OsMFC with $40 \Omega$ resistor and uncontrolled catholyte pH: (A) water flux and RSF and (B) current density.
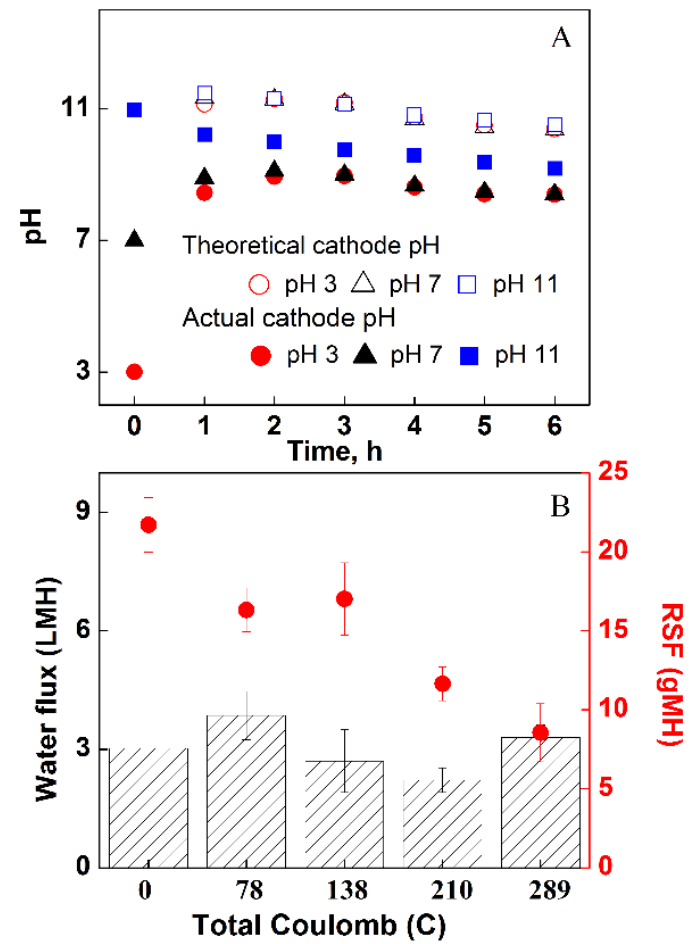

Figure 4.10 The OsMFC performance with uncontrolled catholyte $\mathrm{pH}$ : (A) measured (actual) and calculated $\mathrm{pH}$ value with different initial catholyte $\mathrm{pH}$ (external resistance of $40 \Omega$ ); and (B) water flux and RSF with different external resistance (initial catholyte pH of 7). 
effect on the catholyte $\mathrm{pH}$ due to water flux was very minor, contributing to about 0.2 -unit $\mathrm{pH}$ change in the catholyte. The final catholyte $\mathrm{pH}$ was lower than a theoretical catholyte $\mathrm{pH}$ calculated based on the hydroxide ion production (oxygen reduction reaction) from the transferred charge (Figure 4.10A) (Werner et al. 2013), indicating that the $\mathrm{pH}$ difference could be related to the promoted proton transport. Proton flux could be improved with water flux from the anode to the cathode, which was not considered in the theoretical calculation (Werner et al. 2013). Meanwhile, RSF might stimulate the transport of $\mathrm{OH}^{-}$from the cathode into the anode for charge neutrality, thereby influencing the catholyte $\mathrm{pH}$.

When there was no current generation in the OsMFC (open circuit), the RSF was 18.7 $\pm 1.0,18.2$ \pm 1.0 , and $19.3 \pm 1.1 \mathrm{gMH}$ with the initial catholyte $\mathrm{pH} 3,7$, and 11, respectively (Figure 4.11), indicating that the RSF was not affected by the initial catholyte $\mathrm{pH}$ in an open circuit mode. The open circuit operations excluded the effect of oxygen reduction reaction on RSF, and there was relatively stable anolyte $\mathrm{pH}(\sim 7)$ during the 6-h operation regardless of the initial catholyte $\mathrm{pH}$. In the open circuit mode, the final catholyte $\mathrm{pH}$ was $7.05 \pm 0.12,7.10 \pm 0.07$ and $8.23 \pm 0.05$ when the initial catholyte $\mathrm{pH}$ of 3,7 , and 11 , respectively, indicating that the catholyte $\mathrm{pH}$ significantly changed during the operation, which could be related to the proton/hydroxide transport across the membrane driven by concentration gradients.

RSF was also examined under different current generation and at an uncontrolled catholyte $\mathrm{pH}$ (initially 7). When the external resistance decreased from $100 \Omega$ to $1 \Omega$, the maximum current density increased from $14 \mathrm{~A} \mathrm{~m}^{-3}$ to $89 \mathrm{~A} \mathrm{~m}^{-3}$ and the total Coulomb production increased from 78 C to $289 \mathrm{C}$ (Figure 4.10B). Like the previous test with the controlled catholyte $\mathrm{pH}$, water flux did 
not change significantly with varied total Coulomb, but RSF significantly decreased from $21.7 \pm$ $1.7 \mathrm{gMH}$ to $8.6 \pm 1.8 \mathrm{gMH}$.

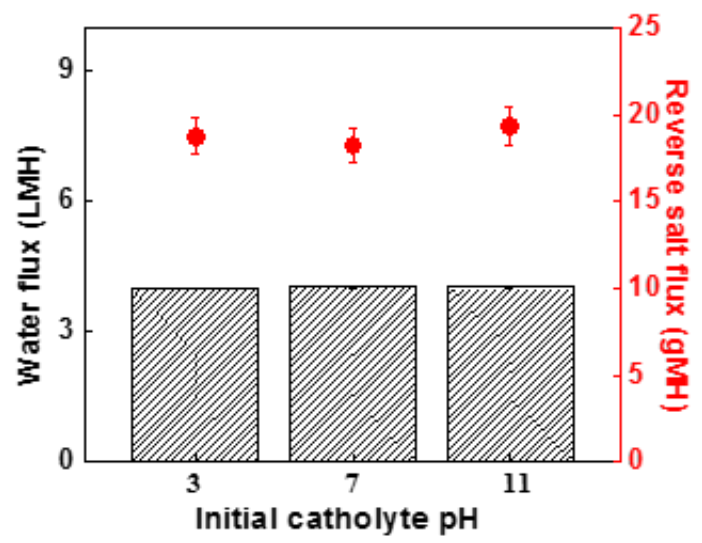

Figure 4.11 Water flux and RSF of the OSMFC with open circuit and uncontrolled catholvte $\mathrm{pH}$.

\subsubsection{Ion transport in the OsMFC}

RSF is the reverse ion transport of draw solute, which includes both $\mathrm{Na}^{+}$and $\mathrm{Cl}^{-}$in the present OsMFC that used sodium chloride as a draw solute. The mechanism of RSF in the OsMFC was investigated by studying the ion transport $\left(\mathrm{Na}^{+}\right.$and $\left.\mathrm{Cl}^{-}\right)$affected by electricity generation and the catholyte $\mathrm{pH}$. The measured concentrations of $\mathrm{Na}^{+}$and $\mathrm{Cl}^{-}$ions were consistent with $\mathrm{RSF}$ calculated from conductivity change. As the total Coulomb production increased from $90 \mathrm{C}$ to 226 C, the transport of $\mathrm{Na}^{+}$decreased from $20.2 \pm 0.1 \mathrm{mmol}$ to $18.7 \pm 0.7 \mathrm{mmol}$, and the transport of $\mathrm{Cl}^{-}$decreased from $17.0 \pm 0.5 \mathrm{mmol}$ to $11.8 \pm 1.6 \mathrm{mmol}$ (Figure 4.12A). When the (controlled) catholyte $\mathrm{pH}$ increased from 3 to 11 , the transport of $\mathrm{Na}^{+}$increased from $18.2 \pm 0.2 \mathrm{mmol}$ to 24.0 $\pm 1.8 \mathrm{mmol}$ and the transport of $\mathrm{Cl}^{-}$increased from $13.1 \pm 0.8 \mathrm{mmol}$ to $20.0 \pm 2.2 \mathrm{mmol}$ (Figure 4.12B). 

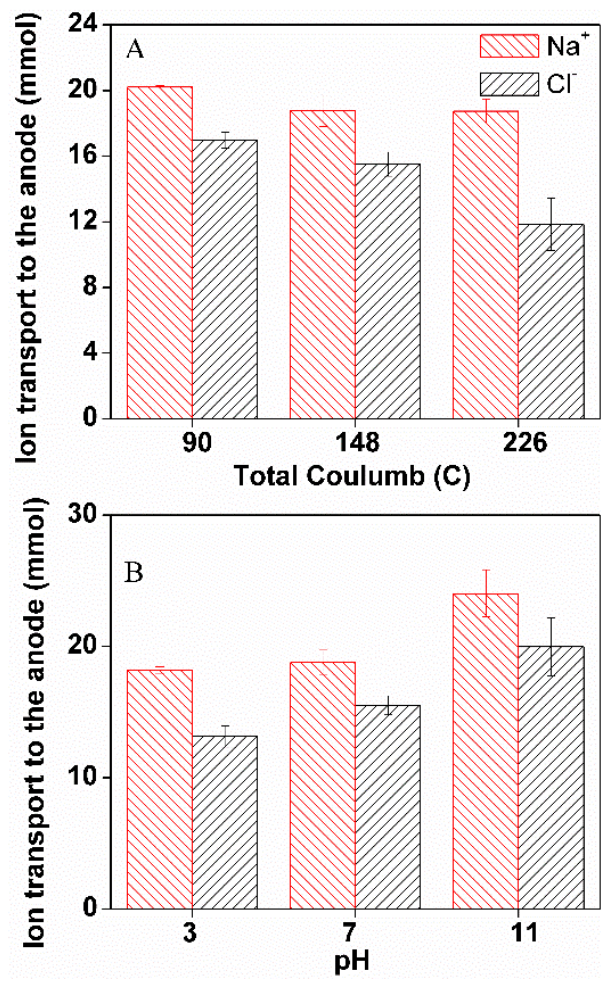

Figure 4.12 Transport of sodium $\left(\mathrm{Na}^{+}\right)$and chloride $\left(\mathrm{Cl}^{-}\right)$ions to the anode: (A) affected by Coulomb production; and (B) affected by the catholyte $\mathrm{pH}$.

One can observe that $\mathrm{Na}+$ and $\mathrm{Cl}$ - ions were not transported at an equal amount, and more $\mathrm{Na}+$ migrated across the FO membrane than Cl-. TFC membrane could behave similarly to a cation exchange membrane when the carboxylic acid groups in the active layer become deprotonated ($\mathrm{COOH}$ becomes -COO-), which will favor the transport of $\mathrm{Na}+$ and hinder the transport of $\mathrm{Cl}-$ (Arena et al. 2015, Arena et al. 2014, Lu et al. 2014a, Tang et al. 2007). The mechanism of ion transport in the present OsMFC was proposed in Figure 4.13. The difference between the transported molar amount of $\mathrm{Na}+$ and $\mathrm{Cl}$ - could be balanced by the forward transport of protons from the anode to the cathode for charge neutrality. When the OsMFC was operated in open circuit, $\mathrm{Na}+$ moved across the TFC membrane from the cathode to the anode due to its high electrostatic attraction to the negatively charged polyamide surface, and dragged the $\mathrm{Cl}$ - across the membrane to maintain the electroneutrality in the anode (Lu et al. 2014a, Phillip et al. 2010). The transport 
of $\mathrm{Na}^{+}$was affected by the current generation in the OsMFC, because electrons moving from the anode electrode to the cathode electrode facilitated the forward transport of $\mathrm{Na}^{+}$(from the anode to the cathode) and inhibited the reverse transport, resulting in a decreased net transport of $\mathrm{Na}^{+}$ from the cathode to the anode. As the total Coulomb production increased from $90 \mathrm{C}$ to $226 \mathrm{C}$, the percentage of the transported $\mathrm{Na}^{+}$decreased from $10.1 \pm 0.5 \%$ to $6.7 \pm 0.3 \%$. The decreased $\mathrm{Na}^{+}$ transport could repel the reversed $\mathrm{Cl}^{-}$transport and thus decrease RSF. As the catholyte $\mathrm{pH}$ increased from 3 to 11, more carboxylic acid functional groups became deprotonated, improving negative charge densities on membrane surface that would facilitate cation transport across the membrane, which positively correlated with RSF.

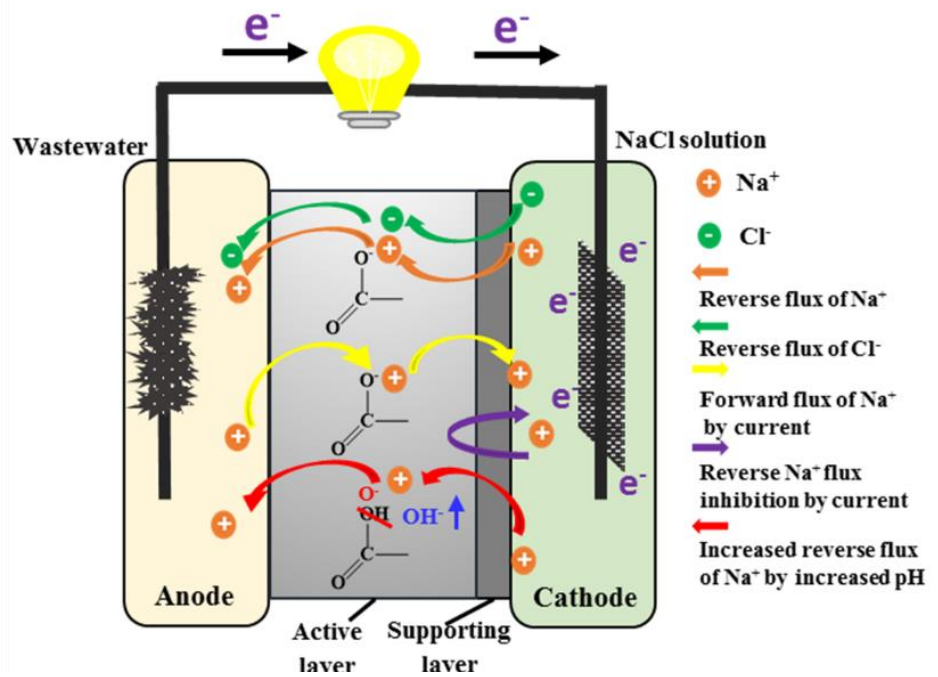

Figure 4.13 Proposed mechanism of the ion transport (orange: $\mathrm{Na}^{+}$; green: $\mathrm{Cl}^{-}$) across the TFC membrane. The arrows represent the flux of ions (orange: reverse flux of $\mathrm{Na}^{+}$; green: reverse flux of $\mathrm{Cl}^{-}$dragged by $\mathrm{Na}^{+}$transport; yellow: forward flux of $\mathrm{Na}^{+}$driven by current; purple: reverse $\mathrm{Na}^{+}$flux inhibited by current; red: increased reverse flux of $\mathrm{Na}^{+}$bv increased catholvte $\mathrm{pH}$ ).

\subsubsection{Mechanism of reduced RSF in the OsMFC}

We further studied the salt transport in two mechanisms, diffusion and EDM, according to NernstPlanck equation. Diffusion was driven by the concentration gradient, while EDM is generated by current or charge difference and can transport certain charged ions (anion/cation) to balance the 
charge. The analysis shows that the percentage of diffusion and EDM was affected by the current generation and catholyte $\mathrm{pH}$ (Fig. 6). In the OsMFC, the electricity generation inhibited the EDM of ions from the cathode to the anode and led to a higher percentage of diffusion. As the total Coulomb production increased from $90 \mathrm{C}$ to $226 \mathrm{C}$, the percentage of EDM decreased from 59.0 $\pm 3.6 \%$ to $26.4 \pm 1.2 \%$ and the percentage of diffusion increased from $41.0 \pm 3.6 \%$ to $73.6 \pm 1.2 \%$ (Figure 4.14A). RSF decreased along with the current generation was likely due to the reduced EDM. As the current generation (total Coulomb production) increased, more electrons flew from the anode to the cathode. The EDM of $\mathrm{Na}^{+}$from the cathode to the anode was in the opposite direction from the electron flow, which would be repulsed and reduced as the increase of electron flow. The contribution of EDM to RSF increased as the catholyte $\mathrm{pH}$ increased (Figure 4.14B), resulting in a lower RSF in the OsMFC. When the (controlled) catholyte $\mathrm{pH}$ increased from 3 to 11, the percentage of EDM increased from $42.1 \pm 5.3 \%$ to $70.1 \pm 1.1 \%$ and the percentage of diffusion decreased from $57.9 \pm 5.3 \%$ to $29.9 \pm 1.1 \%$. When the catholyte $\mathrm{pH}$ decreased, the deprotonated carboxyl groups in the active layer also decreased, leading to a lower charge difference across the membrane and thus a lower EDM.

In this study, the irreversible fouling of FO membrane was excluded, because of the short-term test and frequent membrane cleaning. However, we should acknowledge that membrane fouling could interact with current generation and $\mathrm{pH}$, with consequent impact on RSF. Previous studies have reported controversial conclusions about the influence of membrane fouling on current generation: Werner et al. found that membrane fouling led to water flux decline but the power generation was unaffected, while Ge et al. and Zhu et al. suggested that the membrane fouling could benefit the current generation (Ge and He 2012, Werner et al. 2013, Zhu et al. 2016). The 
membrane fouling could also benefit the forward salt flux, which might result in a reduced RSF (Zhu et al. 2016). Nevertheless, the effects of membrane fouling on current generation and RSF warrant further investigation with actual wastewater and for a long-term operation.
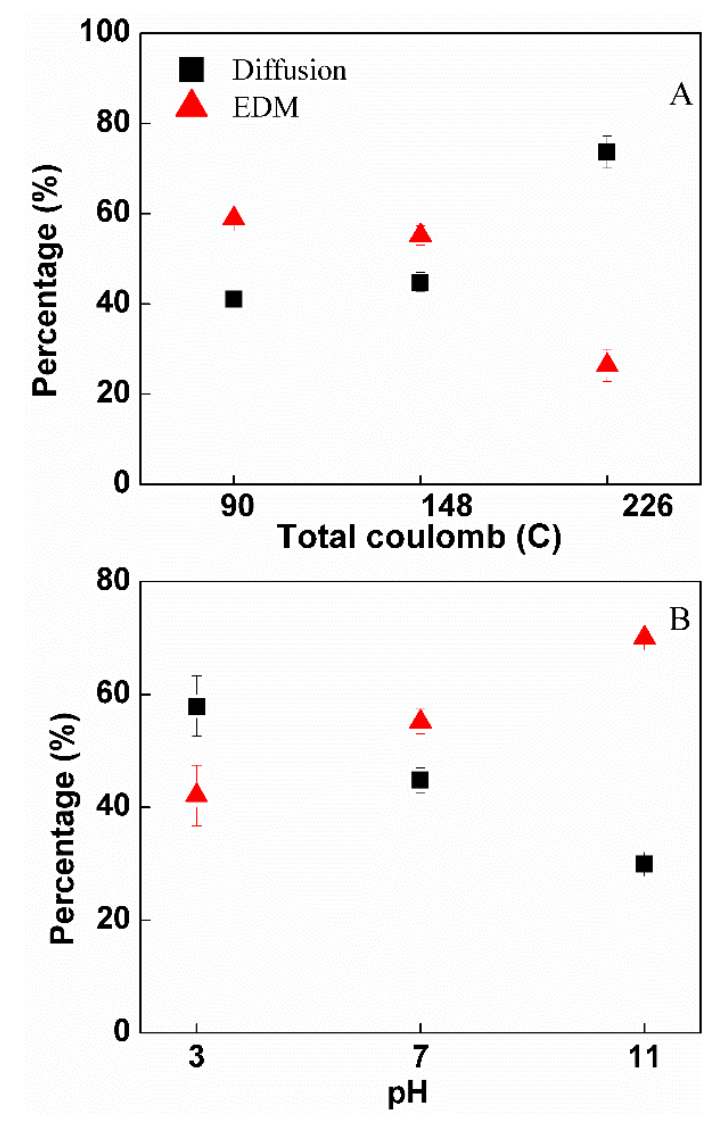

Figure 4.14 Contribution of diffusion and EDM (electricallydriven migration) to ion transport: (A) affected by Coulomb production; and (B) affected by the catholyte $\mathrm{pH}$.

The TFC membrane possesses a cation exchange capability, endowing the function of EDM (electrostatic repulsion) in FO processes when salt ions are used as the draw solutes. However, the electrostatic repulsion in FO membranes is still not well understood (Hancock et al. 2011a, Holloway et al. 2015, Lu et al. 2014a, Phillip et al. 2010). When CTA membrane was employed, Hancock et al. (Hancock et al. 2011a) and Phillip et al. (Phillip et al. 2010) concluded that the driving force for ion transport was only governed by solute diffusion; however, Lu et al. (Lu et al. 
2014a) and Holloway et al. (Holloway et al. 2015) alleged that the electrostatic repulsion is not neglectable in CTA membrane. When TFC membrane was used, the electrostatic repulsion played a more important role in ion transport due to the functional groups (Holloway et al. 2015, Lu et al. 2014a). In the OsMFC with TFC membrane, increased current generation and decreased catholyte $\mathrm{pH}$ could suppress EDM (electrostatic repulsion) and thus decrease RSF. This is a new benefit of the OsMFCs that has not been identified before and could have important implications to OsMFC development and applications. For example, the new benefit of RSF reduction will raise the question whether an OsMFC should be operated under high power output (for energy production) or high current generation (for RSF reduction). This will require detailed analysis of potential tradeoff between energy production and the benefit of RSF reduction. It also implies to the general FO field that electricity may be used to control RSF, but the associated energy input must be clearly understood.

\subsection{Conclusions}

We have successfully achieved reduced RSF in an OsMFC, and this finding can help with developing an effective strategy for controlling RSF, which is one of the major challenges in FObased treatment. The main conclusions and results are summarized as:

- Electricity generation could reduce RSF by more than $75 \%$, compared to that of the open circuit (no electricity generation).

- The catholyte $\mathrm{pH}$ exhibited strong effects on RSF through interaction with current generation, and a lower catholyte $\mathrm{pH}$ would have more RSF inhibition due to higher electricity generation. 
- More $\mathrm{Na}^{+}$migrated across the $\mathrm{FO}$ membrane than $\mathrm{Cl}^{-}$, because that $\mathrm{TFC}$ membrane possessed a certain capacity for cation exchange.

- Two mechanisms, diffusion and electrically-driven migration, were proposed to explain the ion transport across the FO membrane. The electricity generation inhibited the EDM of ions and led to a higher percentage of diffusion. 


\title{
Chapter 5 Ammonium removal from synthetic wastewater promoted by current generation and water flux in an osmotic microbial fuel cell
}

(This section has been published as Qin, M., Hynes, E. A., Abu-Reesh, I. M., \& He, Z.* (2016). Ammonium removal from synthetic wastewater promoted by current generation and water flux in an osmotic microbial fuel cell. Journal of Cleaner Production, 149, 856-862.)

\begin{abstract}
Recovering useful resource from wastes represents a new approach of clean production with significant environmental and economic benefits. Ammonium nitrogen, which is an important inorganic contaminant and also a resource for fertilizer, can be removed and recovered from wastewater. As the first step of recovery, ammonium removal was successfully demonstrated in this study by using an innovative treatment system - osmotic microbial fuel cells (OsMFC). This OsMFC achieved the removal efficiency of $80.1 \pm 2.0 \%$ with an anolyte flow rate of $0.4 \mathrm{~mL} \mathrm{~min}^{-}$ 1. Current generation was a key driving force for ammonium ion movement and increasing current generation from 0 to $1.8 \pm 0.1 \mathrm{~A} \mathrm{~m}^{-2}$ could greatly enhance the removal efficiency from $40.7 \pm 2.4$ $\%$ to $85.3 \pm 3.5 \%$. When current generation was similar, water flux could contribute significantly to facilitating ammonium removal, and as a result the OsMFC exhibited $55.2 \pm 6.5 \%$ higher ammonium removal with water flux of $1.3 \pm 0.2 \mathrm{LMH}$ than that without water flux. In addition, ion diffusion and ion exchange contributed to $17.3 \pm 2.4 \%$ and $2.8 \pm 0.1 \%$ of ammonium removal, respectively, with $35 \mathrm{~g} \mathrm{~L}^{-1} \mathrm{NaCl}$ as a catholyte. The key challenges such as the exact mechanism of ammonium transport, disposal of ammonium residue after recovery, effects of draw solutes, and system scaling up have been identified and discussed. The results of this study will help develop an efficient approach for NEW recovery (NEW: nutrient, energy and water) from wastewater.
\end{abstract}




\subsection{Introduction}

Nitrogen removal from wastewater is of great importance to protect receiving water from eutrophication (Matassa et al. 2015b). The primary form of nitrogen in wastewater is ammonia (Sprynskyy et al. 2005), which can be biologically converted to nitrogen gas via nitrification and denitrification, or anaerobic ammonium oxidation (anammox) (Ahn 2006b, Gupta et al. 2015). Significant energy/chemical input is required by biological nitrogen removal. Meanwhile, ammonia nitrogen is a key fertilizer component for agricultural production, and more than $90 \%$ of the world ammonia is produced by using the Haber-Bosch synthesis process, which consumes 1$2 \%$ of the world energy to synthesize ammonia from hydrogen gas and nitrogen gas under a high pressure (Bicer et al. 2016). Therefore, both the need for ammonia removal from wastewater and the high energy demand for synthesizing ammonia stimulate the interest in recovering ammonia from wastewater, instead of removal, and such recovery will synergistically address the nexus of food, energy, and water.

Ammonia can be recovered from wastewater by using an emerging technology - microbial fuel cells (MFCs) (Arredondo et al. 2015, Kelly and He 2014). In MFCs, organic compounds in wastewater are oxidized by the exoelectrogens growing on an anode electrode and the generated electrons can spontaneously flow from the anode electrode to a cathode electrode for electricity generation (Li et al. 2014a). Electron flow will drive transport of ions between the anode and the cathode to keep electroneutrality, depending on the types of ion exchange membrane used in an MFC. When cation exchange membrane (CEM) is used, cations such as protons, sodium ions and ammonium ions will migrate from the anode into the cathode. As a result of cathode reduction reaction, the $\mathrm{pH}$ of the catholyte could increase to above 11-12, which will facilitate conversion of 
ammonium to ammonia for subsequent recovery by stripping. Ammonia recovery by MFCs has been successfully demonstrated in laboratory studies (Kuntke et al. 2011), and higher current generation would greatly enhance the ammonia recovery (Haddadi et al. 2013, Kuntke et al. 2014). Ammonia can be recovered from various types of ammonium-rich wastewater, such as urine, landfill leachate, and swine wastewater (Kim et al. 2008, Kuntke et al. 2011, Kuntke et al. 2012, Qin et al. 2016b, Zhang et al. 2014a).

To enhance water recovery in MFCs, osmotic microbial fuel cells (OsMFCs) were developed through integrating forward osmosis (FO) with MFCs (Lu et al. 2014b, Zhang et al. 2011a). In OsMFCs, FO membrane instead of CEM is used to separate the anode and the cathode and this semipermeable membrane only permeates water molecules from high water potential to low water potential driven by an osmotic pressure gradient (Cath et al. 2006). Like conventional MFCs, electricity is generated from the oxidation of organics by exoelectrogens in an anode and a terminal electron acceptor such as oxygen is reduced on a cathode catalyzed by catalysts. The difference from conventional MFCs is that OsMFCs can extract high quality water from the anolyte (e.g., wastewater) by using FO membrane. In addition, OsMFCs can generate more electricity under both a batch mode and a continuous mode using either sodium chloride solution or artificial seawater as the catholyte (Ge et al. 2013b, Zhang et al. 2011a). More than $50 \%$ of the treated wastewater could be extracted from the anode of an OsMFC (Ge et al. 2013b, Ismail and Ibrahim 2015). To improve our understanding of enhanced electricity generation in OsMFCs, a mathematical model has been developed and used to predict a reduction of internal resistance with increasing osmotic pressure gradient and water flux, thereby confirming the enhanced current generation (Qin et al. 2015). It was also reported that in an OsMFC, reverse salt flux (RSF), which 
is backward transport of salt ions across the FO membrane into the treated wastewater, was significantly inhibited by the current generation (Qin et al. 2016a).

Higher current generation in OsMFCs encourages the exploration of recovering ammonia, which relies on ammonium transport driven by current generation. The past OsMFC studies focus on recovery of resources such as electricity and water, but ammonia recovery in OsMFCs has not been investigated. Unlike CEM, FO membrane cannot selectively transport ions. However, the unique feature of OsMFCs, water flux, has been found to help improve current generation. Therefore, it will be of great interest to accomplish ammonia recovery in OsMFCs, making OsMFCs a promising technology for "NEW recovery" (NEW: nutrient, energy and water). In this study, we have investigated ammonium removal in a laboratory OsMFC towards understanding of the effects of current generation and water flux on ammonia removal. The recovery of ammonia gas was beyond the scope of this work but it has been performed in various studies via highly efficient absorption in acids (Kuntke et al. 2012, Liu et al. 2016, Wu and Modin 2013). The specific objectives were to: (1) demonstrate the feasibility of ammonium removal in an OsMFC; (2) investigate the effect of the current generation and water flux on ammonium removal; and (3) explore the mechanism of ammonium removal in the OsMFC.

\subsection{Materials and Methods}

\subsubsection{OsMFC setup}

The OsMFC consisted of two equal-size compartments $\left(14 \times 7 \times 3.7 \mathrm{~cm}^{3} /\right.$ ach with a liquid volume of $280 \mathrm{~mL}$ ) as the anode and the cathode, respectively, separated by a TFC FO membrane (Hydration Technology Innovations, LLC, Albany, OR, USA) with its active layer facing the 
anode (feed) side. Plastic mesh was placed on both sides of the FO membrane as support materials. Two carbon brushes (Gordon Brush Mfg. Co. Inc., Commerce, CA, USA) were pretreated by being soaked in pure acetone overnight and heat-treated in a muffle furnace (Model 550 Isotemp Series, Fisher Scientific, Pittsburgh, PA, USA) at $450{ }^{\circ} \mathrm{C}$ for 30 minutes; after pretreatment they were inserted into the anode compartment as the anode electrodes. The cathode electrode was a piece of carbon cloth coated with Platinum (Pt) as the catalyst for oxygen reduction reaction $(0.3 \mathrm{mg} \mathrm{Pt} \mathrm{cm}$

${ }^{2}$ ). Before use, the TFC membrane was soaked in deionized (DI) water for $30 \mathrm{~min}$ according to the manufacturer's instruction (Stillman et al. 2014). The surface area of the FO membrane was about $98 \mathrm{~cm}^{2}$.

\subsubsection{OsMFC operation}

The OsMFC was operated at room temperature of $\sim 21{ }^{\circ} \mathrm{C}$. The anode compartment was inoculated with anaerobic sludge from the Peppers Ferry Regional Wastewater Treatment Plant (Radford, VA, USA). To mimic the digestion effluent of livestock waste (Park et al. 2010), the anode influent solution was prepared containing (per liter of deionized water): sodium acetate, $1.5 \mathrm{~g} ; \mathrm{NH}_{4} \mathrm{Cl}, 3.0$ $\mathrm{g} ; \mathrm{NaHCO}_{3}, 2.0 \mathrm{~g} ; \mathrm{NaCl}, 0.15 \mathrm{~g} ; \mathrm{MgSO}_{4}, 0.005 \mathrm{~g} ; \mathrm{CaCl}_{2}, 0.006 \mathrm{~g}$; and trace elements solution, 1 $\mathrm{mL}$ (Angenent and Sung 2001). The initial concentration of COD in the anode influent was 1200 $\mathrm{mg} \mathrm{L}{ }^{-1}$ while the initial concentration of ammonium nitrogen $\left(\mathrm{NH}_{4}{ }^{+}-\mathrm{N}\right)$ was $780 \mathrm{mg} \mathrm{L}^{-1}$. Sodium chloride was used as the draw solute for water extraction. To avoid the difference in performance caused by reactor manufacturing, the same OsMFC reactor was used for all the tests. The OsMFC was operated in a continuous mode. The 2-L bottles were connected to the anode and cathode compartments as reservoirs and placed on digital balance for monitoring water flux. The cathode compartment was aerated with the air to provide oxygen for cathode reaction and create flow turbulence. To study the effects of current generation, the external resistance was adjusted to four 
levels ( $10 \Omega, 20 \Omega, 40 \Omega$ and quasi-infinite or open circuit mode), and the catholyte was $35 \mathrm{~g} \mathrm{~L}^{-1}$ $\mathrm{NaCl}$. In the experiments of different water flux, the concentration of the draw solution (catholyte) was varied at $2 \mathrm{~g} \mathrm{~L}^{-1}, 10 \mathrm{~g} \mathrm{~L}^{-1}$ and $35 \mathrm{~g} \mathrm{~L}^{-1}$. The effect of the anoltye flow rate on ammonium removal was investigated with $35 \mathrm{~g} \mathrm{~L}^{-1} \mathrm{NaCl}$ as the catholyte at different flow rates: $0.2 \mathrm{~mL} \mathrm{~min}$ ${ }^{1}, 0.3 \mathrm{~mL} \mathrm{~min}{ }^{-1}$ and $0.4 \mathrm{~mL} \mathrm{~min}^{-1}$. The effect of water flux on ammonium removal was examined in both closed-circuit condition and open-circuit condition with different catholyte $\mathrm{NaCl}$

concentrations $\left(2 \mathrm{~g} \mathrm{~L}^{-1}\right.$ and $\left.35 \mathrm{~g} \mathrm{~L}^{-1}\right)$. The ammonium removal with different water flux and same current generation was studied with two operations: (1) $2 \mathrm{~g} \mathrm{~L}^{-1} \mathrm{NaCl}$ catholyte and $10 \Omega$ external resistor; and (2) $35 \mathrm{~g} \mathrm{~L}^{-1} \mathrm{NaCl}$ catholyte and $88 \Omega$ resistor. The ammonium transported to the cathode side could be recovered as ammonia gas, which has been demonstrated in our previous studies (Liu et al. 2016, Qin and He 2014). The present study focused on the ammonium removal, and the recovery was not performed.

\subsubsection{Measurement and analysis}

The OsMFC voltage was recorded every 2 minutes by a digital multimeter (2700, Keithley Instruments Inc., Cleveland, OH, USA). The polarization curve was performed by using a potentiostat (Reference 600, Gamry Instruments, Warminster, PA, USA) at a scan rate of $0.2 \mathrm{mV}$ $\mathrm{s}^{-1}$. The $\mathrm{pH}$ was measured by using a benchtop $\mathrm{pH}$ meter (Oakton Instruments, Vernon Hills, IL, USA). The conductivity was measured by using a benchtop conductivity meter (Mettler-Toledo, Columbus, OH, USA). The volumetric densities of power and current were calculated based on the liquid volume of the anode compartment, according to a previous study (Zhang et al. 2010). The concentrations of chemical oxygen demand (COD) and ammonium nitrogen $\left(\mathrm{NH}_{4}{ }^{+}-\mathrm{N}\right)$ were measured using a DR/890 colorimeter (HACH Co., Ltd., USA) according to the manufacturer's 
instruction. The catholyte weight in the reservoir was recorded every 1 min on a digital balance (Scort Pro, Ohous, Columbia, MD, USA) (Zou and $\mathrm{He} 2016$ ). The concentrations of $\mathrm{Na}^{+}$and $\mathrm{Cl}^{-}$ were quantified by using ion chromatography (Dionex LC20 ion chromatograph, Sunnyvale, CA, U.S.A.) equipped with an ED40 electrochemical detector. The COD removal efficiency $\left(C O D_{\text {Removal }}\right)$ was calculated as:

$\operatorname{COD}_{\text {Removal }}=1-\frac{C_{\text {effluent }} \times Q_{\text {effluent }}}{C_{\text {influent }} \times Q_{\text {influent }}}$

where $C_{\text {effluent }}$ and $C_{\text {influent }}$ are the COD for effluent and influent (mol L $\left.{ }^{-1}\right)$, respectively. $Q_{e f f l u e n t}$ and $Q_{\text {influent }}$ are the flow rate of anode effluent and anode influent $\left(\mathrm{L} \mathrm{min}^{-1}\right)$, respectively.

Water flux $J_{w}$ across the FO membrane $\left(\mathrm{L} \mathrm{m}^{-2} \mathrm{~h}^{-1}, \mathrm{LMH}\right)$ was calculated by the weight change of catholyte solution over time (Wang et al. 2010b).

$J_{w}=\frac{V_{c, t}-V_{c, 0}}{A_{m} t}$

(Equation 5.2)

where $V_{c, t}$ and $V_{c, o}$ are the volume (L) of the catholyte at the test time $t$ (h) and 0 , respectively. The difference between $V_{c, t}$ and $V_{c, 0}$ equals to the weight increase of the draw solution in value $(\mathrm{kg})$ during this period; $A_{m}$ is the membrane area $\left(\mathrm{m}^{2}\right)$.

\subsection{Results and Discussion}

\subsubsection{Feasibility of ammonium removal across FO membrane}

To examine the feasibility of ammonium removal, the OsMFC was operated with varied $\mathrm{NaCl}$ concentrations in the catholyte. Current density increased from $1.1 \pm 0.1 \mathrm{~A} \mathrm{~m}^{-2}$ to $2.6 \pm 0.1 \mathrm{~A} \mathrm{~m}^{-2}$ (mean value \pm standard deviation) when the $\mathrm{NaCl}$ concentration increased from $2 \mathrm{~g} \mathrm{~L}^{-1}$ to $35 \mathrm{~g} \mathrm{~L}^{-1}$ (Figure 5.1A). Both the membrane resistance and catholyte resistance are related to the reciprocal of catholyte solution conductivity (Qin et al. 2015). A higher $\mathrm{NaCl}$ concentration could increase 
the catholyte conductivity and decrease both the membrane resistance and catholyte resistance, thereby increasing current generation. In addition, the OsMFC with $35 \mathrm{~g} \mathrm{~L}^{-1} \mathrm{NaCl}$ had a higher COD removal efficiency $(80.7 \pm 1.5 \%)$ than that with $2 \mathrm{~g} \mathrm{~L}^{-1} \mathrm{NaCl}(64.7 \pm 1.9 \%)$, benefited from the higher current generation. The ammonium removal efficiency was $23.5 \pm 3.5 \%, 34.3 \pm 5.3 \%$, and $52.5 \pm 4.7 \%$, with 2,10 , and $35 \mathrm{~g} \mathrm{~L}^{-1}$, respectively (Figure 5.1B). When the catholyte $\mathrm{NaCl}$ concentration was $2 \mathrm{~g} \mathrm{~L}^{-1}$, there was no water flux $(0 \pm 0.1 \mathrm{LMH})$ (Figure 5.1B). As the increase of catholyte $\mathrm{NaCl}$ concentration, the osmotic pressure difference $(\Delta \pi$, calculated from the salt concentration in two chambers) between the anode (feed) and the cathode (draw) increased and therefore, the water flux from anode to the cathode increased to $0.7 \pm 0.1 \mathrm{LMH}$ and $1.3 \pm 0.2 \mathrm{LMH}$ with a $\mathrm{NaCl}$ concentration of $10 \mathrm{~g} \mathrm{~L}^{-1}$ and $35 \mathrm{~g} \mathrm{~L}^{-1}$, respectively. Such an increase in water flux would help with ammonium removal, which will be discussed in the following sections

\subsubsection{Effects of anolyte flow rate}

The anolyte flow rate is a key operating factor and could affect the loading rates of both organic

compounds and ammonium. When the anolyte flow rate was $0.2 \mathrm{~mL} \mathrm{~min} \mathrm{~m}^{-1}, 0.3 \mathrm{~mL} \mathrm{~min} \mathrm{~m}^{-1}$ and 0.4 

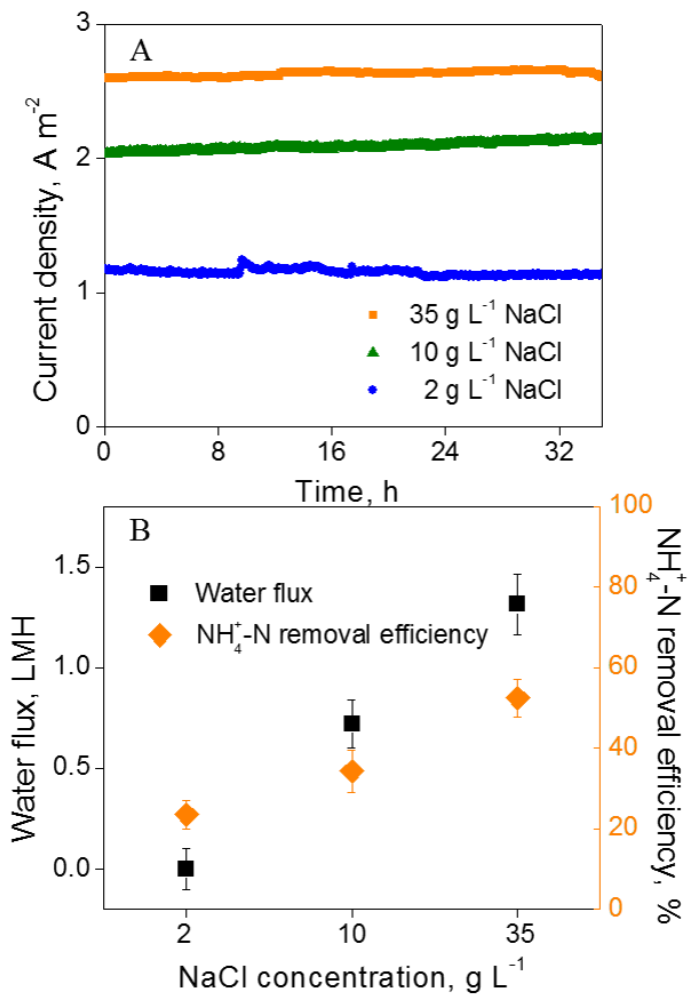

Figure 5.1 Ammonium removal by the OsMFC affected by different catholyte $\mathrm{NaCl}$ concentrations: (A) current generation; and (B) water flux and $\mathrm{NH}_{4}{ }^{+}-\mathrm{N}$ removal efficiency.

$\mathrm{mL} \mathrm{min}^{-1}$, the ammonium loading rate was $0.5,1.0$, and $1.5 \mathrm{mmol} \mathrm{h}^{-1}$, respectively. During the test, the catholyte $\mathrm{NaCl}$ concentration was maintained at $35 \mathrm{~g} \mathrm{~L}^{-1}$. Varying the anolyte flow rate also changed organic input, resulting in increased current generation with a higher flow rate. For example, current density increased from $1.8 \pm 0.1$ to $2.6 \pm 0.1 \mathrm{~A} \mathrm{~m}^{-2}$, when the anolyte flow rate was changed from 0.2 to $0.4 \mathrm{~mL} \mathrm{~min}^{-1}$ (Figure 5.2A). However, the COD removal efficiency decreased as the anolyte flow rate increased: $91.3 \pm 2.3 \%, 85.2 \pm 1.1 \%$, and $80.7 \pm 1.5 \%$ with three flow rates. The ammonium removal efficiency was clearly affected by the anolyte flow rate. Increasing the anolyte flow rate from 0.2 to $0.4 \mathrm{~mL} \mathrm{~min}^{-1}$ decreased the ammonium removal efficiency from $85.3 \pm 3.5 \%$ to $52.5 \pm 4.7 \%$ (Figure $5.2 \mathrm{~A}$ ). Assuming one electron generated from the oxidation of organic substrates in the anode moves one ammonium ion from the anode into the cathode for charge balance, the transport of ammonium ions would contribute to $69.3 \pm 1.9 \%$, 
$78.0 \pm 3.1 \%$, and $80.1 \pm 2.0 \%$ of ionic flux through the FO membrane with the anolyte flow rate of $0.2,0.3$ and $0.4 \mathrm{~mL} \mathrm{~min}{ }^{-1}$, respectively. Thus, a higher anolyte flow rate would improve the importance of ammonium ions as a proton shuttle in the OsMFC (Cord-Ruwisch et al. 2011). Water flux was not changed significantly with varied anolyte flow rates $(p>0.05$, Figure 5.2B), likely due to the small change in osmotic pressure difference under those conditions.
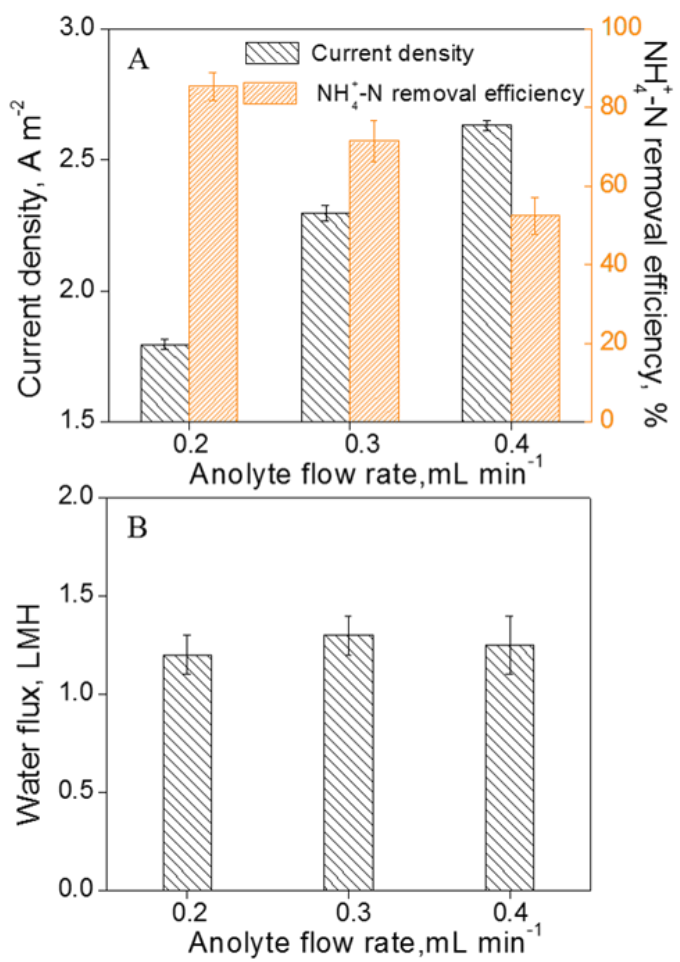

Figure 5.2 Ammonium removal by the OsMFC affected by different anolyte flow rates: (A) current generation and $\mathrm{NH}_{4}{ }^{+}-\mathrm{N}$ removal efficiency; and (B) water flux.

\subsubsection{Effects of current generation}

Current generation (or electron flow) is a key driving force for ammonium transport, and its importance in the OsMFC was examined under the conditions of varied external resistance. When applying a $10 \Omega$ external resistor and $35 \mathrm{~g} \mathrm{~L}^{-1} \mathrm{NaCl}$ as the catholyte, current density was $1.8 \pm 0.1$ $\mathrm{A} \mathrm{m}^{-2}$ (Figure 5.3A), and the ammonium removal efficiency was $85.3 \pm 3.5 \%$ (Fig. 3B). For 
comparison, the OsMFC under the open-circuit condition did not generate any current and removed $40.7 \pm 2.4 \%$ of ammonium nitrogen (Figure 5.3B). Further comparison was conducted with $2 \mathrm{~g} \mathrm{~L}^{-1} \mathrm{NaCl}$ as the catholyte, which led to no water flux due to the lack of osmotic pressure gradient. Likewise, current generation, although lower at $0.8 \pm 0.1 \mathrm{~A} \mathrm{~m}^{-2}$ than that with $35 \mathrm{~g} \mathrm{~L}^{-1}$ catholyte, increased the ammonium removal efficiency from $21.4 \pm 2.2 \%$ (open circuit condition) to $47.8 \pm 2.2 \%$ (Figure 5.3B). Those results have confirmed the critical role of current generation in driving ammonium ions out of the anolyte and moving through the FO membrane. In addition to current-driven movement, ammonium ions could also migrate due to the ammonium concentration gradient and ion exchange, which resulted in ammonium removal under the open circuit condition.
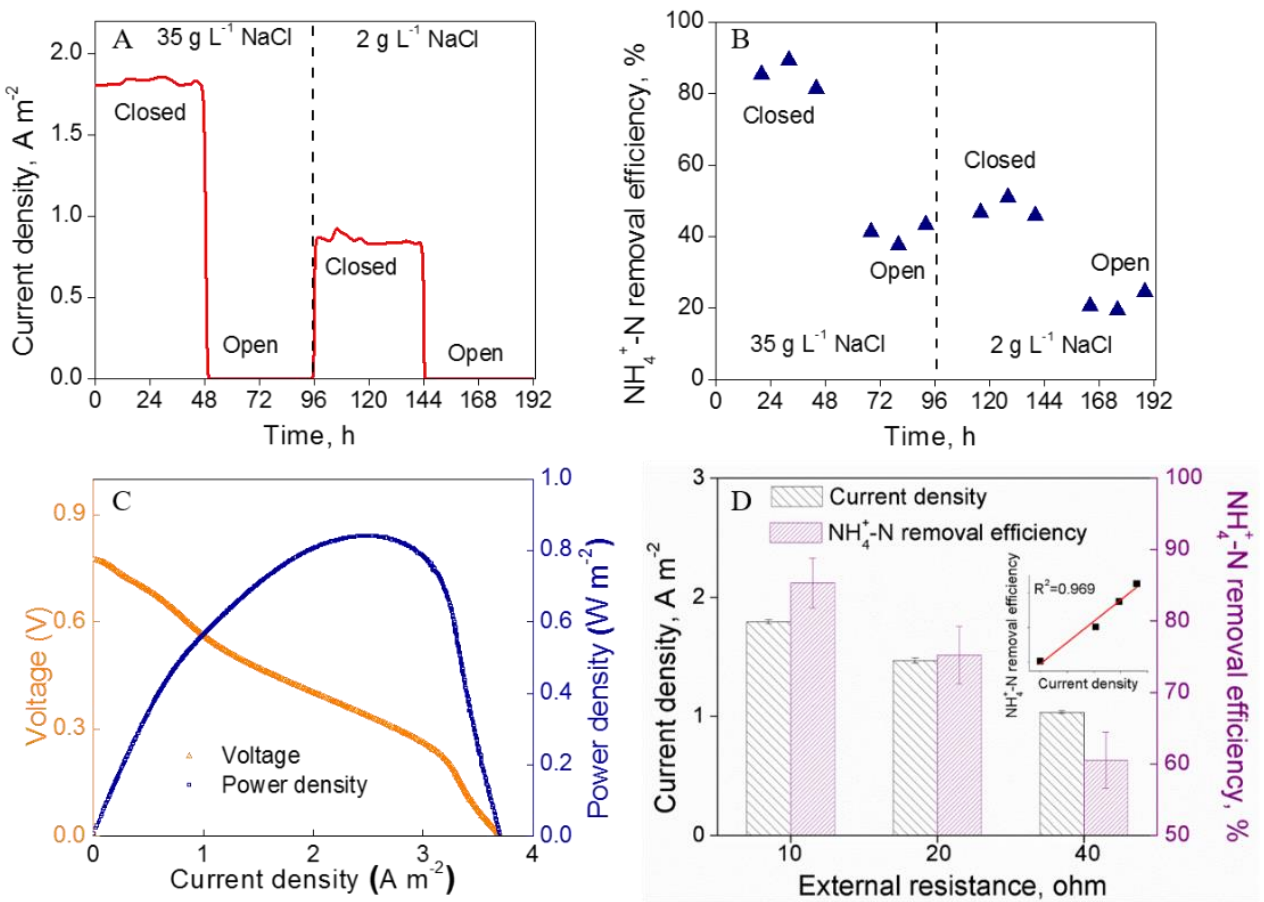

Figure 5.3 Ammonium removal by the OsMFC affected by the current generation: (A) current generation in open and closed circuit conditions; (B) $\mathrm{NH}_{4}{ }^{+}-\mathrm{N}$ removal efficiency in closed and open circuit conditions; (C) polarization curve; and (D) current generation and $\mathrm{NH}_{4}{ }^{+}-\mathrm{N}$ removal efficiency with different external resistances. 
More external resistances were examined to reveal the relationship between ammonium removal and current generation in the OsMFC. This was investigated at a fixed ammonium loading rate of $0.5 \mathrm{mmol} \mathrm{h}^{-1}$ and fixed catholyte $\mathrm{NaCl}$ concentration of $35 \mathrm{~g} \mathrm{~L}^{-1}$. As shown in the polarization curve (Figure 5.3C), the open circuit voltage of the OsMFC was $0.78 \mathrm{~V}$ with an estimated internal resistance of $19.2 \Omega$. Therefore, the external resistance was varied from $10 \Omega$ to $40 \Omega$ to represent different current generation including the condition of the maximum power output. As expected, current density increased from $1.0 \pm 0.1 \mathrm{~A} \mathrm{~m}^{-2}$ to $1.8 \pm 0.1 \mathrm{~A} \mathrm{~m}^{-2}$ when the external resistance decreased from $40 \Omega$ to $10 \Omega$ (Figure 5.3D). Ammonium removal clearly increased from $60.5 \pm 3.9$ $\%(40 \Omega)$ to $85.3 \pm 3.5 \%(10 \Omega)$ with increasing current generation (Figure 5.3D). The highest ammonium removal efficiency with $10 \Omega$ doubles that under an open circuit condition. A linear relationship between current generation and ammonium removal efficiency was observed $\left(\mathrm{R}^{2}\right.$ of 0.969, inset, Figure 5.3D).

\subsubsection{Effects of water flux}

Water flux is playing an important role in the OsMFC because it can exert effects on water extraction, current generation and ion transport (and thus ammonium removal). To examine the effect of water flux, we investigated the ammonium removal in the presence or absence of water flux. To obtain the same (or similar) current generation (so that we can exclude the effect of current generation), two conditions were created: first, $2 \mathrm{~g} \mathrm{~L}^{-1} \mathrm{NaCl}$ catholyte and $10 \Omega$ external resistor; and second, $35 \mathrm{~g} \mathrm{~L}^{-1} \mathrm{NaCl}$ catholyte and $88 \Omega$ resistor. The current densities of those two conditions were very similar at $0.9 \pm 0.1 \mathrm{~A} \mathrm{~m}^{-2}$ (Figure 5.4A). However, they exhibited very different water flux, $0 \pm 0.1 \mathrm{LMH}$ in the first condition and $1.3 \pm 0.2 \mathrm{LMH}$ in the second condition, resulting in different ammonium removal efficiency of $47.8 \pm 2.2 \%$ and $74.2 \pm 4.3 \%$, respectively (Figure 
5.4B). This demonstrates that water flux could promote ammonium transport from the anolyte into the catholyte. Some evidence might also be obtained from the prior results under the open circuit condition: the OsMFC with water flux (with $35 \mathrm{~g} \mathrm{~L}^{-1} \mathrm{NaCl}$ as the catholyte) achieved 40.7 $\pm 2.4 \%$ of ammonium removal, much higher than $21.4 \pm 2.2 \%$ without water flux (with $2 \mathrm{~g} \mathrm{~L}^{-1}$ $\mathrm{NaCl}$ as the catholyte) (Figure 5.3B). A previous study has reported that the cation flux could be increased when sodium was in the feed solution and ammonium was in the draw solution in FO process, resulting from the ion exchange of cation ions ( $\mathrm{Lu}$ et al. 2014a). But bioelectricity generation in an OsMFC could greatly inhibit back flux of cations (e.g., sodium ions) from the draw into the feed (Qin et al. 2016a) and facilitate forward movement of ammonium from the feed into the draw. Our analysis showed that ion exchange was not a major contributor to ammonium movement in the present OsMFC (more details in next section).
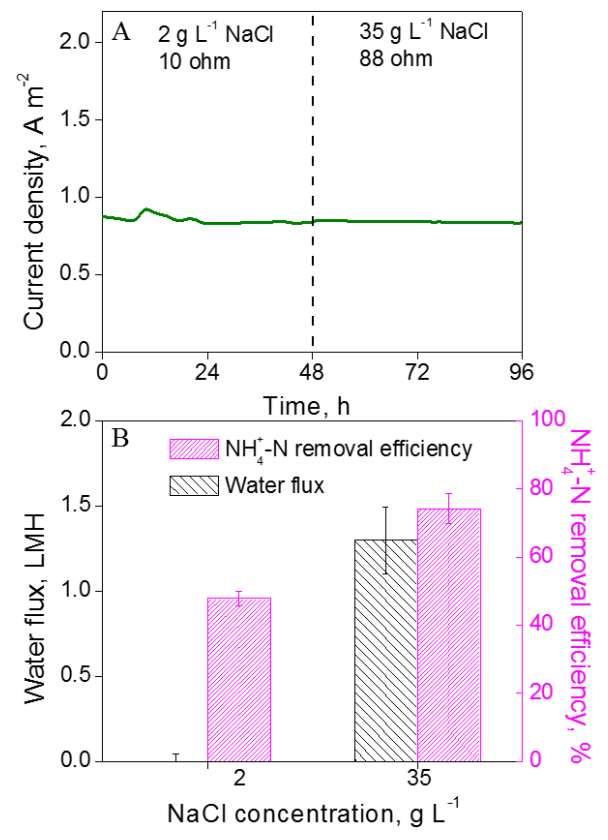

Figure 5.4 Ammonium removal by the OsMFC affected by water flux: (A) similar current generation under two different conditions, $2 \mathrm{~g} \mathrm{~L}^{-1} \mathrm{NaCl}$ as catholyte / $10 \Omega$ external resistance and $35 \mathrm{~g} \mathrm{~L}^{-1} \mathrm{NaCl}$ as catholyte / $88 \Omega$ external resistance; and (B) $\mathrm{NH}_{4}{ }^{+}-\mathrm{N}$ removal efficiency and water flux under those two conditions. 


\subsubsection{Mechanisms of ammonium movement}

Despite the important role of current generation and water flux in ammonium removal, there also other mechanisms contributing to ammonium transport in the present OsMFC, such as ion diffusion and ion exchange. Thus, in total we identified four mechanisms of ammonium transport: (1) diffusion of ammonium ions due to a concentration gradient; (2) ammonium ion movement from the anode to the cathode promoted by water flux; (3) ion exchange between anode ammonium ion and cathode sodium ions; and (4) migration of ammonium ions driven by current generation for charge balance. Those contributions were analyzed for improving our understanding (Figure 5.5), but it should be noted that precise quantification is very difficult at this time because of simultaneous function of multiple mechanisms.

When the OsMFC was operated with $2 \mathrm{~g} \mathrm{~L}^{-1} \mathrm{NaCl}$ catholyte in an open-circuit mode, there was neither current generation nor water flux and therefore, the ammonium removal was mainly attributed to the diffusion of ammonium ions and the ion exchange between sodium and ammonium (Figure 5.5A). Based on the data of reverse fluxed sodium ions, the contribution of ion exchange was estimated to be only $0.4 \pm 0.1 \%$. Thus, the dominant transport mechanism in the absence of both water flux and electricity generation would be diffusion, which caused $21.0 \pm 2.2$ $\%$ of ammonium removal from the anode. We assumed the same diffusion contribution in other conditions due to the same ammonium gradient across the FO membrane, although some variation would be expected and warrant further investigation. A higher catholyte salinity of $35 \mathrm{~g} \mathrm{~L}^{-1}$ in the 
open circuit mode stimulated water flux, which contributed to $17.3 \pm 2.4 \%$ of ammonium transport, and improved the ion exchange contribution to $\sim 3 \%$.
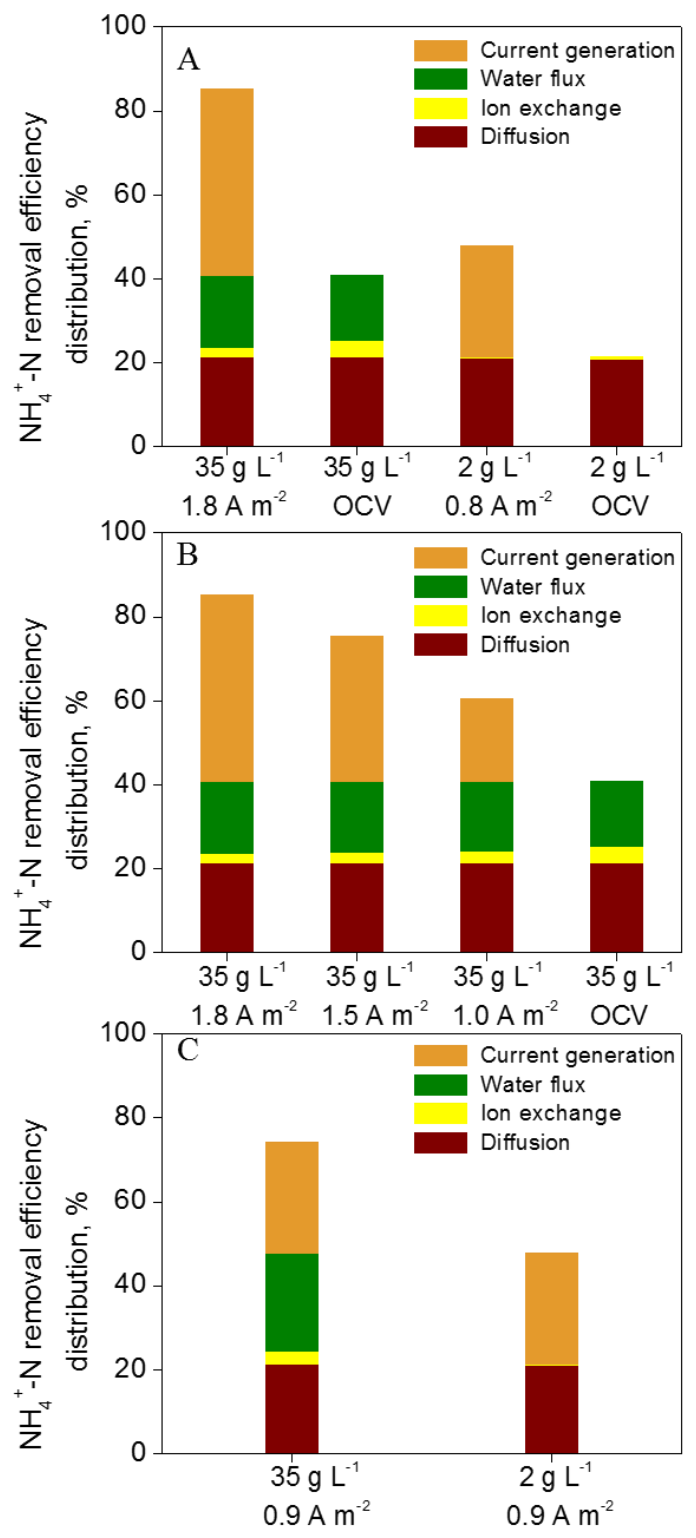

Figure 5.5 The contribution of different mechanisms to ammonium removal: (A) comparison between open and closed circuit conditions with or without water flux; (B) effects of current generation; and (C) effects of water flux (orange: current generation; green: water flux; yellow: ion exchange; and maroon: diffusion). 
Current generation exhibited a significant contribution of $47.0 \pm 3.2 \%\left(35 \mathrm{~g} \mathrm{~L}^{-1}\right)$ and $26.4 \pm 2.2$

$\%\left(2 \mathrm{~g} \mathrm{~L}^{-1}\right)$. Increasing current density from $1.0 \pm 0.1$ to $1.8 \pm 0.1 \mathrm{~A} \mathrm{~m}^{-2}$ also enhanced its contribution from $19.8 \pm 3.9 \%$ to $47.0 \pm 3.2 \%$ (Figure 5.5B). It was observed that current generation decreased the contribution of ion exchange from $3.9 \pm 0.1 \%$ (open circuit mode) to 2.3 $\pm 0.1 \%\left(1.8 \pm 0.1 \mathrm{~A} \mathrm{~m}^{-2}\right)$. The contribution of water flux to ammonium removal was distinguished in the condition of similar current generation (Figure 5.5C). The contribution of current generation for both operations was similar at $\sim 26 \%$. The condition of $35 \mathrm{~g} \mathrm{~L}^{-1} \mathrm{NaCl}$ catholyte and $88-\Omega$ external resistance had additional $26.4 \pm 4.4 \%$ of ammonium removal, including $23.4 \pm 4.4 \%$ from water flux and $3.0 \pm 0.2 \%$ due to ion exchange. It was reported that proton flux could be enhanced in an OsMFC, compared to that of an MFC with cation exchange membrane (Zhu et al. 2015); ammonium ions have been considered as a proton shuttle (Cord-Ruwisch et al. 2011) and thus, water flux may also promote the transport of ammonium ions.

\subsubsection{Implications and perspectives}

The present study, for the first time, demonstrated the feasibility of ammonium removal in an OsMFC that could be extended to nitrogen recovery from wastewater (Kelly and He 2014). This new benefit - nitrogen removal/recovery of the OsMFC, in addition to energy production and water recovery, will create more application niches and improve technological sustainability. For example, wastewater containing a large amount of ammonium, such as digester effluent, animal wastewater, urine, and landfill leachate, can be treated in the OsMFC for contaminant removal and resource recovery. To further develop OsMFCs towards practical applications, several challenges must be properly addressed. First, the exact mechanism of water flux promoted ammonium transport should be better understood. This is important to integrate nitrogen recovery with water recovery in an FO-based system. Second, although we did not perform ammonia recovery in the 
present study, our prior work has shown that recovery (via ammonia stripping) would not be $100 \%$ efficient and there will be remaining ammonium in the catholyte (as high as 100-200 $\mathrm{mg} \mathrm{L}^{-1}$ ) (Qin and He 2014). This ammonium residue can affect the quality of the produced water, and must be removed, for instance, during draw solute regeneration. Third, it is not clear how ammonium transport/removal will be affected by the type of draw solute in an OsMFC. Especially, when a nitrogen-based draw solute such as ammonium bicarbonate (McCutcheon et al. 2006) or fertilizer (Phuntsho et al. 2011) is used for the purpose of easy regeneration, a smaller concentration gradient across the FO membrane could decrease ammonium transport from the anode into the cathode and thus lower the removal. In addition, RSF of nitrogen-based draw solute may also reduce ammonium removal efficiency. Last, system scaling up of the OsMFC and examination with actual wastewater for a long-term performance (for investigating system stability and membrane fouling) will be necessary and important.

\subsection{Conclusions}

Ammonium removal has been successfully achieved in an OsMFC. A higher anolyte flow rate would improve the transport of ammonium ions because of more organic inputs and thus higher current generation. The study confirmed that current generation was also a key factor to drive ammonium movement across FO membrane. The unique feature of the OsMFC (compared to conventional MFCs) - water flux was found to be able to promote ammonium transport from the anolyte into the catholyte. In addition to current generation and water flux, ion diffusion and ion exchange also contributed to ammonium movement. The findings of this study will help develop OsMFCs as a sustainable technology for resource recovery from wastewater. 


\title{
Chapter 6 Understanding Ammonium Transport in Bioelectrochemical Systems towards its Recovery
}

(This section has been published as Liu, Y., Qin, M. (Co-first author), Luo, S., He, Z., * \& Qiao, R.* (2016) Understanding Ammonium Transport in Bioelectrochemical Systems towards its Recovery. Scientific Reports, 6, 22547-22557.)

\begin{abstract}
We report an integrated experimental and simulation study of ammonia recovery using microbial electrolysis cells (MECs). The transport of various species during the batch-mode operation of a MEC was examined experimentally and the results were used to validate the mathematical model for such an operation. It was found that, while the generated electrical current through the system tends to acidify (or basify) the anolyte (or catholyte), their effects are buffered by a cascade of chemical groups such as the $\mathrm{NH}_{3} / \mathrm{NH}_{4}{ }^{+}$group, leading to relatively stable $\mathrm{pH}$ values in both anolyte and catholyte. The transport of $\mathrm{NH}_{4}{ }^{+}$ions accounts for $\sim 90 \%$ of the total current, thus quantitatively confirming that the $\mathrm{NH}_{4}{ }^{+}$ions serve as effective proton shuttles during MEC operations. Analysis further indicated that, because of the Donnan equilibrium at cation exchange membrane-anolyte/catholyte interfaces, the $\mathrm{Na}^{+}$ion in the anolyte actually facilitates the transport of $\mathrm{NH}_{4}{ }^{+}$ions during the early stage of a batch cycle and they compete with the $\mathrm{NH}_{4}{ }^{+}$ions weakly at later time. These insights, along with a new and simple method for predicting the strength of ammonia diffusion from the catholyte toward the anolyte, will help effective design and operation of BES-based ammonia recovery systems.
\end{abstract}




\subsection{Introduction}

Nitrogen removal from wastewater is of great importance to protect receiving water from eutrophication (Matassa et al. 2015b). The primary form of nitrogen in wastewater is ammonia (or as a product of decomposing organic nitrogen) (Sprynskyy et al. 2005), and thus removing ammonia from municipal and industrial wastewater prior to discharge is obligatory. Ammonia can be removed from wastewater using biological or physicochemical methods such as nitrification and denitrification, ion exchange, air stripping, and anaerobic ammonium oxidation (anammox) (Ahn 2006a, Gupta et al. 2015). Because ammonia nitrogen is a key fertilizer component for agricultural applications, recovery instead of removal of ammonia from wastewater can greatly impact on both sustainable wastewater management and economics. An emerging approach for recovering ammonia is through bioelectrochemical systems (BES) (Arredondo et al. 2015, Kelly and He 2014).

In BES-based ammonia recovery systems, organic compounds in wastewater are oxidized by the exoelectrogens growing on an anode, producing a current through its external circuit (Wang and Ren 2013). When cation exchange membranes (CEMs) are used (Cheng et al. 2013, Haddadi et al. 2013, Logan et al. 2008b, Rozendal et al. 2006a), the current is carried by cations such as $\mathrm{NH}_{4}{ }^{+}$ ions moving from the anolyte into the catholyte through the CEM. The $\mathrm{NH}_{4}{ }^{+}$ions, once transported into the catholyte, are converted into $\mathrm{NH}_{3}$ molecules because the catholyte is usually rendered basic by the production of $\mathrm{OH}^{-}$ions therein (Haddadi et al. 2014, Kim et al. 2008, Kuntke et al. 2012). Some of these $\mathrm{NH}_{3}$ molecules can then be recovered through gas aeration. This type of

process has been demonstrated in various BES including microbial fuel cells (MFCs) and microbial electrolysis cells (MECs). In MECs, a higher current density would greatly enhance 
ammonia recovery (Haddadi et al. 2013), and thus MECs with external power exhibit a better performance for ammonium recovery than MFCs (Zhang et al. 2014a). It was reported that ammonia can be recovered from ammonium-rich wastewater, such as synthetic wastewater, urine, and swine wastewater (Kim et al. 2008, Kuntke et al. 2011, Kuntke et al. 2012, Qin and He 2014).

While a general picture of ammonia recovery using BES is emerging, some issues remain open. In particular, the transport of $\mathrm{NH}_{4}{ }^{+}$ions and $\mathrm{NH}_{3}$ is not fully understood despite their essential role in ammonia recovery. Some studies indicate that $\mathrm{NH}_{4}{ }^{+}$transport dominates the current across the CEM (i.e., for each electron passing through the external circuit, $\sim 1 \mathrm{NH}_{4}{ }^{+}$ion moves from the anolyte into the catholyte through the CEM) (Cord-Ruwisch et al. 2011), while other studies suggest that $\mathrm{NH}_{4}{ }^{+}$ions may carry only $40 \%$ of the total current (Arredondo et al. 2015, Desloover et al. 2012, Kuntke et al. 2011, Kuntke et al. 2012). In addition, whether $\mathrm{NH}_{4}{ }^{+}$ions move through the CEM via diffusion or migration is not clear. A recent study suggests that the migration of $\mathrm{NH}_{4}^{+}$ ions dominates over their diffusion through a comparison of the net $\mathrm{NH}_{4}{ }^{+}$transport under the close and open circuit conditions (Haddadi et al. 2013). However, this conclusion was based on the assumption that the diffusion of $\mathrm{NH}_{4}{ }^{+}$ions under these conditions is the same, whose validity/generality is not yet clear. In a seminal simulation study in which key transport phenomena and chemical reaction within the BES are modeled comprehensively, it was found that the diffusion of $\mathrm{NH}_{3}$ from the catholyte to the anolyte can greatly reduce the efficiency of recovery (Dykstra et al. 2014c). However, the condition under which such diffusion is important has not been delineated yet. Finally, since many cations such as $\mathrm{Na}^{+}$ions exist in the anolyte, for a given current through the BES, they may compete with $\mathrm{NH}_{4}{ }^{+}$ions for transport across the CEM (Kim et 
al. 2007b, Kuntke et al. 2011, Rozendal et al. 2006a). How strong such competition is and how it affects the ammonia recovery is not well understood.

Another open issue is how the $\mathrm{pH}$ in the anolyte and catholyte is regulated. Since the $\mathrm{pH}$ values in the anolyte/catholyte affect how the total nitrogen content is partitioned between its two forms $\left(\mathrm{NH}_{4}{ }^{+}\right.$ions and $\left.\mathrm{NH}_{3}\right)$, and the latter in turn affects the transport of $\mathrm{NH}_{4}{ }^{+}$ions through the CEM and the recovery of $\mathrm{NH}_{3}$ through aeration, it is essential to regulate these $\mathrm{pH}$ effectively. Furthermore, regulating the anolyte $\mathrm{pH}$ is also essential for ensuring microbial activity and hence the reliable operation of BES (Cheng et al. 2013, He et al. 2008, Zhang et al. 2011b). Maintaining a basic environment with $\mathrm{pH}>9$ in the catholyte facilitates the conversion of $\mathrm{NH}_{4}{ }^{+}$ions into $\mathrm{NH}_{3}$, which is key for the effective recovery of ammonia by aeration. The effective control of the $\mathrm{pH}$ in the anolyte and the catholyte requires a thorough understanding of what governs the $\mathrm{pH}$ in these electrolytes (Jadhav and Ghangrekar 2009, Kim et al. 2007b, Rozendal et al. 2006a). While it is known that the generation of proton in the anolyte and hydroxide ions in the catholyte plays an important role in controlling the pH (Dykstra et al. 2014c, Jadhav and Ghangrekar 2009, Kim et al. 2007b, Kuntke et al. 2014, Kuntke et al. 2012, Rozendal et al. 2006a), how and to what extent the transport of all ions and their chemical reactions with each other affect the $\mathrm{pH}$ is little understood.

Resolving the above issues using experimental study alone is difficult due to the challenge in assessing details of all transport processes in the BES. Numerical modeling can address this challenge, but most prior modeling of BES focused on power production and organic removal (Peng et al. 2013, Picioreanu et al. 2007, Ping et al. 2014, Qin et al. 2015, Zeng et al. 2010). In the 
only comprehensive model for BES-based ammonia recovery, the transport and chemical reactions of major species in BES was studied at a steady state (Dykstra et al. 2014c). The predictions of this model agree qualitatively with experimental observations and they led to critical insight into the effects of current density and membrane properties on the ammonia recovery. However, the model has not been validated by detailed comparison with experiments, and studies based on this model did not cover the issues described above, e.g., the competition of inert ions (i.e., ions that do not react with other species within the system, e.g., the $\mathrm{Na}^{+}$ions) with the $\mathrm{NH}_{4}{ }^{+}$ions for transport across the CEM cannot be studied using steady state models. Here, we have studied the ammonia recovery in a representative BES - microbial electrolysis cells, by integrating experiments with simulations.

\subsection{Materials and Methods}

\subsubsection{MEC setup}

A bench-scale cubic shape MEC was used in this experiment. Both the anode and the cathode chambers had the same dimension of $9 \mathrm{~cm} \times 4.7 \mathrm{~cm} \times 0.8 \mathrm{~cm}$. The liquid volume of the anode chamber was $200 \mathrm{~mL}$, while that of the cathode chamber was $180 \mathrm{~mL}$. A CEM (CMI-7000, Membrane International Inc., Glen Rock, NJ, USA) with a sectional area of $42.3 \mathrm{~cm}^{2}$ was used to separate anode and cathode chamber. The anode electrode was a carbon brush (Gordon Brush Mfg. Co., Inc., CA, USA) and the cathode electrode was a piece of $32-\mathrm{cm}^{2}$ carbon cloth (Zoltek Companies, Inc., MO, USA) that was coated with $5 \mathrm{mg} \mathrm{cm}^{-2}$ of Pt/C (10\% wt. Pt on Carbon Vulcan, Fuel Cell Earth LLC, USA). The anode was inoculated with the anaerobic sludge from the Peppers Ferry Regional Wastewater Treatment Plant (Radford, VA, USA). To mimic the digestion effluent of livestock waste (Park et al. 2010), the anode influent solution was prepared containing (per liter of deionized water): sodium acetate, $1.5 \mathrm{~g}$; $\mathrm{NH}_{4} \mathrm{Cl}, 3.0 \mathrm{~g} ; \mathrm{NaHCO}_{3}, 2.0 \mathrm{~g}$; 
$\mathrm{NaCl}, 0.15 \mathrm{~g} ; \mathrm{MgSO}_{4}, 0.005 \mathrm{~g} ; \mathrm{CaCl}_{2}, 0.006 \mathrm{~g}$; and trace elements solution (Angenent and Sung 2001), $1 \mathrm{~mL}$. The cathode chamber was initially filled with $180 \mathrm{~mL}$ of deionized water. An external voltage of $0.8 \mathrm{~V}$ was applied to the circuit by a power supply (CSI3644A, Circuit Specialists, Inc., Mesa, AZ, USA) according to a previous study (Yossan et al. 2013). The MEC was operated in a batch mode at room temperature $\left(\sim 20^{\circ} \mathrm{C}\right)$. The anolyte was partially replaced $(75 \%)$ every $48 \mathrm{~h}$ while the catholyte was resupplied to initial volume $(180 \mathrm{~mL})$ when a new batch cycle started. Both the anolyte and catholyte were recirculated at a flow rate of $20 \mathrm{~mL} \mathrm{~min}{ }^{-1}$. The aeration rate was $375 \mathrm{~mL} \mathrm{~min}^{-1} .2 \mathrm{~mL}$ sample were collected regularly from both chambers for measurement and $1 \mathrm{M} \mathrm{H}_{2} \mathrm{SO}_{4}$ was used to absorb the stripped $\mathrm{NH}_{3}$ gas from cathode.

\subsubsection{Measurement and analysis}

The voltage across a $1-\Omega$ resistor (R) in the MEC circuit was recorded every 2 min by a digital multimeter (2700, Keithley Instruments Inc., Cleveland, OH, USA). The pH of liquid stream was measured by two benchtop pH meters installed in the anode and the cathode, respectively (Oakton Instruments, Vernon Hills, IL, USA and Accumet AB250, Fisher Scientific, Pittsburgh, PA, USA). The concentrations of chemical oxygen demand (COD) and ammonium nitrogen $\left(\mathrm{NH}_{4}{ }^{+}-\mathrm{N}\right)$ were measured using a DR/890 colorimeter (HACH Co., Ltd., USA) according to manufacturer's instruction. Ionic concentrations (e.g., $\mathrm{Na}^{+}, \mathrm{Cl}^{-}$) were quantified by using ion chromatography (Dionex LC20 ion chromatograph, Sunnyvale, CA, U.S.A.) equipped with an ED40 eletrochemical detector. The acetate concentration was measured in the anolyte and the catholyte samples that were filtered through 0.22 um PVDF membrane filter and by high-performance liquid chromatorgraphy (HPLC) (Shimadzu, Columbia, MD), equipped with an Aminex HPX-87H column (Bio-Rad, Hercules, CA) and refractive index detector (RID, 10A, Shimadzu). The HPLC 
column was kept at $65^{\circ} \mathrm{C}$, and $0.5 \mathrm{mM}$ sulfuric acid solution was used as a mobile phase at flow rate of $0.6 \mathrm{~mL} \mathrm{~min}^{-1}$.

\subsubsection{Mathematical model of BES-based ammonia recovery}

We extend the previous steady state theory (Dykstra et al. 2014c) to model the ammonia recovery in BES operating in the batch mode. The model considers the transport of various species across the CEM and the acid-base reactions within the entire system. Without loss of generality, the following species are included: $\mathrm{Na}^{+}, \mathrm{Cl}^{-}, \mathrm{HAc}, \mathrm{Ac}^{-}, \mathrm{NH}_{4}{ }^{+}, \mathrm{NH}_{3}, \mathrm{H}_{2} \mathrm{CO}_{3}, \mathrm{HCO}_{3}{ }^{-}, \mathrm{CO}_{3}{ }^{2-}, \mathrm{H}^{+}$and $\mathrm{OH}^{-}$ . The system is divided into three parts: the anode chamber, the cathode chamber, and the CEM (Figure 6.1). The anode and the cathode chambers are both (but separately) treated in a lumped way, and the evolution of the average concentration of a species $i$ inside each chamber follows

$$
V_{j} \frac{\partial C_{i}^{j}}{\partial t}=A \cdot J_{i, j}^{\prime}-\dot{R}_{i, j}^{a}+\dot{S}_{i, j}^{c} V_{j}
$$

where $V_{j}$ is the volume of chamber $j\left(j=1\right.$ : anode chamber; $j=2$ cathode chamber), $C_{i}^{j}$ is the concentration of species $i$ in chamber $j, A$ is the area of the CEM, and $J_{i, j}^{\prime}$ is the flux of species $i$ into chamber $j . \dot{R}_{i, j}^{a}$ is the removal of species $i$ (e.g., $\mathrm{CO}_{2}, \mathrm{NH}_{3}$ etc., acetate removal due to the aeration is neglected, since it's concentration in the catholyte is always low, as shown in the study) from chamber $j$ due to aeration, which is determined by assuming fast equilibrium between species

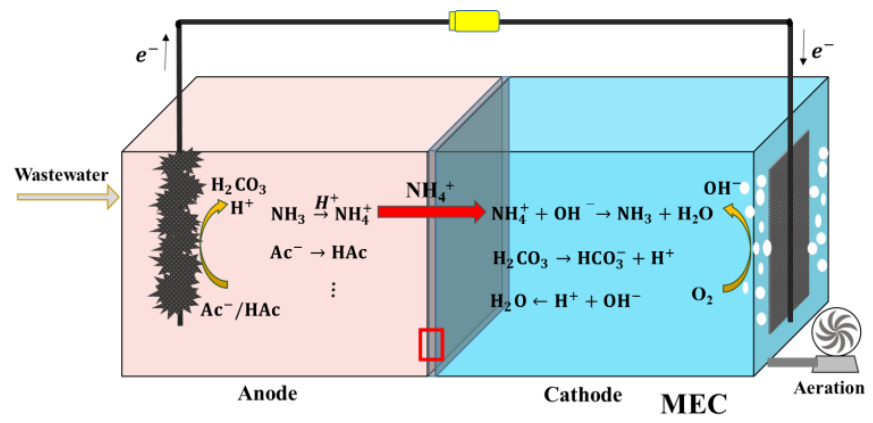

Figure 6.1 A sketch of the MEC-based ammonia recovery system. 
dissolved in the catholyte and existing in the aeration gas. Previous study had shown that this assumption works well. (Dykstra et al. 2014c, Powers et al. 1987) For example, the removal of $\mathrm{NH}_{3}$ from the cathode chamber, $\dot{R}_{N_{3}, 2}^{a}$, is given by $C_{\mathrm{NH}_{3}}^{2} Q_{\text {inert }} / p_{\text {tot }} K_{\mathrm{H}, \mathrm{NH}}$, where $Q_{\text {inert }}$ and $p_{\text {tot }}$ are the volumetric flow rate and pressure of the inert aeration gas, respectively. $K_{\mathrm{H}, \mathrm{NH}}$ is the Henry's constant for $\mathrm{NH}_{3}$ in water. $\dot{S}_{i, j}^{c}$ is the generation/removal rate (per volume) of the species $i$ due to chemical/biological reactions, respectively. Briefly, we consider the consumption of $\mathrm{Ac}^{-}$ by the microbes, the acid-base equilibrium among different species (e.g., $\mathrm{NH}_{4}^{+} \leftrightarrows \mathrm{NH}_{3}+\mathrm{H}^{+}$, HAc $\left.\leftrightarrows \mathrm{Ac}^{-}+\mathrm{H}^{+}, \ldots\right)$. A list of all reactions considered can be found in Table 6.1 in the Supplementary Information. According to the previous work (Dykstra et al. 2014c), we assume that the acid-base reactions are fast so that the chemical equilibrium between the species involved in these reactions is always maintained.

Table 6.1 Equilibrium constants for acid-base reactions and aqueous-solution equilibrium

\begin{tabular}{|c|c|c|c|c|c|}
\hline 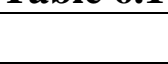 & Constant & Reaction & Equilibrium equation & Value* & Ref. \\
\hline \multirow{5}{*}{$\begin{array}{l}\text { Acid- } \\
\text { base } \\
\text { reaction }\end{array}$} & $\mathrm{K}_{\mathrm{a}, \mathrm{Ac}}$ & $\mathrm{HAc} \leftrightarrow \mathrm{Ac}^{-}+\mathrm{H}^{+}$ & $K_{\mathrm{a}, \mathrm{Ac}}=\frac{C_{\mathrm{Ac}^{-}} \cdot C_{\mathrm{H}^{+}}}{C_{\mathrm{HAc}}}$ & 0.0174 & $\begin{array}{l}\text { (Dykstr } \\
\text { a et al. } \\
2014 b)\end{array}$ \\
\hline & $\mathrm{K}_{\mathrm{a}, \mathrm{CA} 1}$ & $\mathrm{H}_{2} \mathrm{CO}_{3} \leftrightarrow \mathrm{H}^{+}+\mathrm{HCO}_{3}^{-}$ & $K_{\mathrm{a}, \mathrm{CA} 1}=\frac{C_{\mathrm{H}^{+}} \cdot C_{\mathrm{HCO}_{3}^{-}}}{C_{\mathrm{H}_{2} \mathrm{CO}_{3}}}$ & $4.4668 \times 10^{-4}$ & $\begin{array}{l}\text { (Dykstr } \\
\text { a et al. } \\
2014 b)\end{array}$ \\
\hline & $\mathrm{K}_{\mathrm{a}, \mathrm{CA} 2}$ & $\mathrm{HCO}_{3}{ }^{-} \leftrightarrow \mathrm{H}^{+}+\mathrm{CO}_{3}{ }^{2-}$ & $K_{\mathrm{a}, \mathrm{CA} 2}=\frac{C_{\mathrm{H}^{+}} \cdot C_{\mathrm{CO}_{3}^{2-}}}{C_{\mathrm{HCO}_{3}^{-}}}$ & $4.6774 \times 10^{-8}$ & $\begin{array}{l}\text { (Dykstr } \\
\text { a et al. } \\
2014 b)\end{array}$ \\
\hline & $\mathrm{K}_{\mathrm{a}, \mathrm{NH}}$ & $\mathrm{NH}_{4}^{+} \leftrightarrow \mathrm{NH}_{3}+\mathrm{H}^{+}$ & $K_{\mathrm{a}, \mathrm{NH}}=\frac{C_{\mathrm{H}^{+}} \cdot C_{\mathrm{NH}_{3}}}{C_{\mathrm{NH}_{4}^{+}}}$ & $5.6234 \times 10^{-7}$ & $\begin{array}{l}\text { (Dykstr } \\
\text { a et al. } \\
2014 \mathrm{~b})\end{array}$ \\
\hline & $\mathrm{K}_{\mathrm{w}}$ & $\mathrm{H}_{2} \mathrm{O} \leftrightarrow \mathrm{H}^{+}+\mathrm{OH}^{-}$ & $K_{\mathrm{w}}=C_{\mathrm{H}^{+}} \cdot C_{\mathrm{OH}^{-}}$ & $1 \times 10^{-8}$ & $\begin{array}{l}\text { (Dykstr } \\
\text { a et al. } \\
2014 b)\end{array}$ \\
\hline $\begin{array}{l}\text { Henry's } \\
\text { constant }\end{array}$ & $\mathrm{K}_{\mathrm{H}, \mathrm{CO}_{2}}$ & & $K_{\mathrm{H}, \mathrm{CO}_{2}}=\frac{C_{\mathrm{CO}_{2}, \mathrm{aq}}}{p_{\mathrm{CO}_{2}}}$ & 33.46 & $\begin{array}{c}\text { (Dykstr } \\
\text { a et al. } \\
2014 b \text {, } \\
\text { Persat } \\
\text { et al. } \\
2009)\end{array}$ \\
\hline
\end{tabular}




\begin{tabular}{|l|l|l|l|l|l|}
\hline & $\mathrm{K}_{\mathrm{H}, \mathrm{NH}_{3}}$ & & $K_{\mathrm{H}_{\mathrm{NH}}}=\frac{C_{\mathrm{NH}_{3}, \mathrm{aq}}}{p_{\mathrm{NH}_{3}}}$ & 56250 & $\begin{array}{c}\text { (Dykstr } \\
\text { a et al. } \\
2014 \mathrm{~b})\end{array}$ \\
\hline
\end{tabular}

*The values correspond to concentration unit of $\mathrm{mol} / \mathrm{m}^{3}$ and pressure unit of atm;

The CEM is resolved spatially in its thickness direction ( $x=0$ and $L$ correspond to the CEM-anolyte and CEM-catholyte interfaces, respectively). Each species $i$ at $x=0$ and $L$ is always in equilibrium with that in the anode and cathode chambers, respectively (see below for more details). The distribution of each species $i$ across the CEM, $C_{i}^{m}$, is governed by

$$
\frac{\partial C_{i}^{m}}{\partial t}=\dot{S}_{i, m}^{c}+\nabla \cdot J_{i, m}
$$

where $\dot{S}_{i, m}^{c}$ is the generation/consumption of species $i$ by acid-base equilibriums and is determined in the same way as in the electrode chambers. $J_{i, m}$ is the flux of species $i$ in the membrane given by the Nernst-Planck equation (Dykstra et al. 2014c, Higa et al. 1998, Sata 2004):

$$
J_{i, m}=-D_{i}^{m}\left(\frac{\partial C_{i}^{m}}{\partial x}+z_{i} C_{i}^{m} \frac{e}{k_{B} T} \frac{\partial \phi}{\partial x}\right)
$$

where $D_{i}^{m}$ and $z_{i}$ are the diffusion coefficient in the membrane and valence of species $i$, respectively; $e$ and $k_{B} T$ are the electron charge and thermal energy; $\phi$ is the electrical potential. Because the concentration of various species inside the anode/cathode chambers evolves slowly at a time scale of tens of hours but the relaxation of species concentration within the CEM is fast due to CEM's small thickness, the time dependence term in Equation 6.2 is unimportant and thus dropped hereafter.

The concentration distribution of any species $i$ across the anolyte/catholyte-CEM interfaces is treated as follows. For electrically neutral species, their concentrations are continuous across these 
interfaces. For a charged species $i$, we adopt the Donnan equilibrium condition (Dykstra et al. 2014c, Galama et al. 2013, Higa et al. 1998), i.e.,

$$
C_{i}^{\mathrm{m}}=\exp \left(-z_{i} e \Delta \phi / k_{B} T\right) C_{i}^{j}
$$

where $\Delta \phi$ is the electrical potential drop across the CEM-solution interface (usually termed the Donnan potential). Note that the Donnan potential attract cations into (repel anions from) the CEM so that the negative fixed charge inside the membrane is balanced by the free ions.

Finally, the electro-neutrality condition and the charge conservation law are enforced:

$$
\begin{gathered}
\omega X+\Sigma_{i} Z_{i} C_{i}=0 \\
I=F \Sigma_{i} z_{i} J_{i}
\end{gathered}
$$

where $\omega$ is the sign of the fixed space charge in any domain $(\omega=0$ in the anode/cathode chamber and zero charge membranes; $\omega=-1$ and +1 in cation and anion exchange membranes, respectively). $X$ is the fixed charge density of each domain. $F$ and $I$ are the Faraday constant and the current density through the CEM.

Equations (Equation 6.1)-(Equation 6.6), along with the equations for various chemical reactions, form a complete model of the ammonia recovery operation using BES. These nonlinear, timedependent equations were solved using MATLAB. In our simulations, all design and operation parameters of the MEC, e.g., the volume of electrode chamber, aeration rate, and current density (Figure 6.2a), are taken from that in the experiments unless otherwise mentioned. For the CEM (CMI-7000) used in this system, $X$ was determined to be 5M using the method established in prior studies (Aeshala et al. 2013, Dykstra et al. 2014c, Sarmidi 2009, Sata 2004). The diffusion coefficient of various species in the CEM is usually smaller than that in bulk solutions (Chen and 
Ruckenstein 2015, Dykstra et al. 2014c). The diffusion coefficients of all species are either taken from prior experimental data or by fitting the current experimental results. In the latter case, care was taken to ensure that the fitted data are within the range expected for ion diffusion in typical CEMs (Dykstra et al. 2014c, Haddadi et al. 2013, Kim et al. 2007b, Stenina et al. 2004). A summary of all parameters used in these simulations is provided in Table 6.2, Table 6.3, and Table 6.4 .
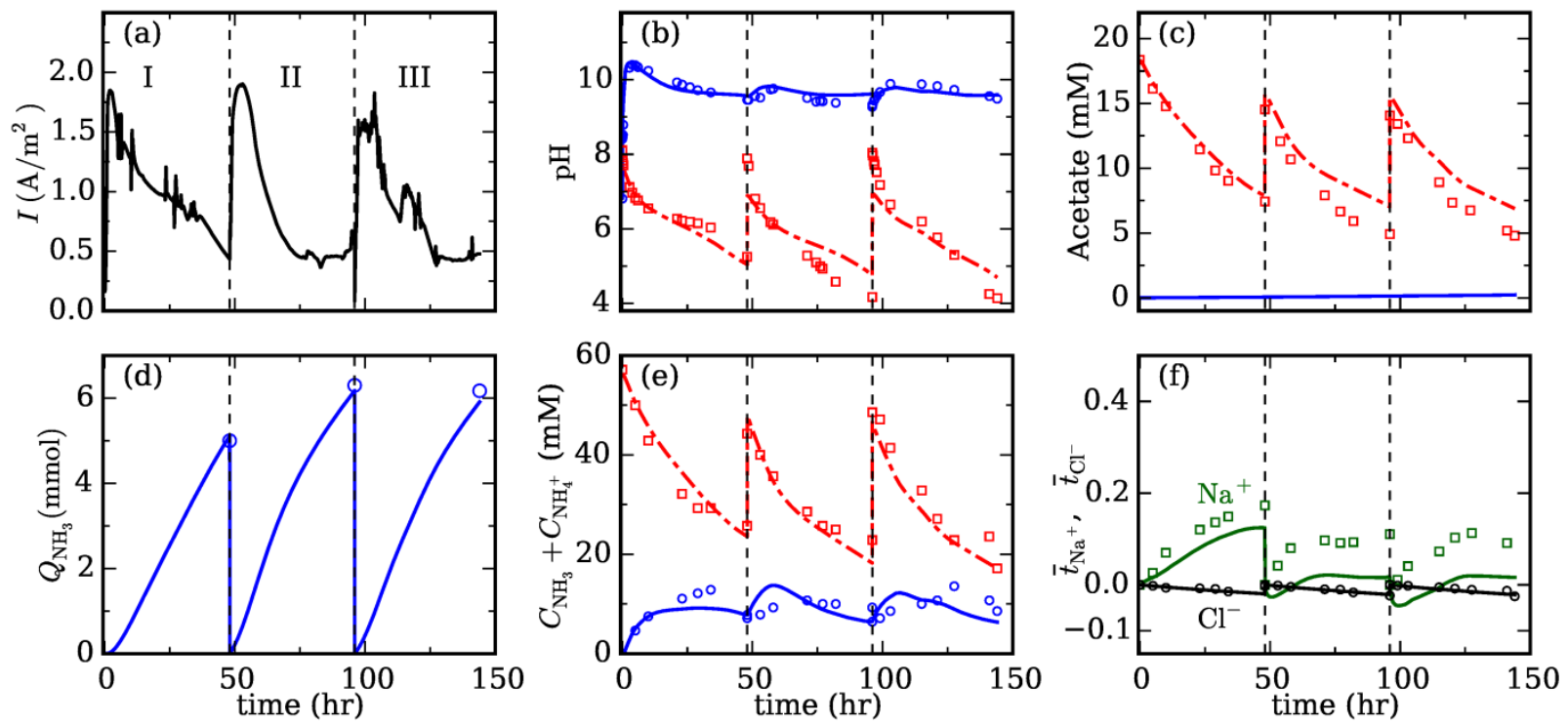

Figure 6.2 Comparison of experimental and model studies of ammonia recovery during three batch cycles of MEC operation. The current density measured experimentally (a) is feed into the models to predict the various observables of the anolyte and the catholyte: the $\mathrm{pH}(\mathrm{b})$, the acetate concentration (c), the amount of $\mathrm{NH}_{3}$ collected from catholyte by aeration (d), the total nitrogen content (e), and the transport of $\mathrm{Na}^{+}$and $\mathrm{Cl}^{-}$ions out of the anolyte (f). Symbols are the experiment data and lines are model prediction. In (b-e), red lines are for anolyte and blue lines are for catholyte. In (c), the acetate concentration in catholyte is below the detection limit of our equipment and thus not shown. In (f), to highlight the relative importance of $\mathrm{Na}^{+} / \mathrm{Cl}^{-}$ion transport in the overall charge transport in the system, their transport is quantified using the cumulative transport number $\bar{t}_{i}^{+}$(see text, $\bar{t}_{i}^{+}=1.0$ means that the charge carried by the transport a species $i$ is equal to the total charge passed through the membrane). A detailed summary of operating conditions and parameters (e.g., initial species concentration in anolyte/catholyte) is in provided in Table 6.2, Table 6.3, and Table 6.4.

Table 6.2 System configuration

\begin{tabular}{|l|l|l|}
\hline Parameters & Values & Notes \\
\hline $\mathrm{A}\left(\mathrm{m}^{2}\right)$ & $42.3 \times 10^{-4}$ & Membrane area \\
\hline $\mathrm{V}_{1}\left(\mathrm{~m}^{3}\right)$ & $0.2 \times 10^{-3}$ & Volume of anode chamber \\
\hline $\mathrm{V}_{2}\left(\mathrm{~m}^{3}\right)$ & $0.18 \times 10^{-3}$ & Volume of cathode chamber \\
\hline
\end{tabular}




\begin{tabular}{|l|l|l|}
\hline $\mathrm{L}(\mathrm{m})$ & $4.75 \times 10^{-4}$ & Membrane thickness \\
\hline $\mathrm{X}(\mathrm{M})$ & 5 & Membrane's fixed charge concentration (CEM-7000) \\
\hline$J_{\text {inert }}\left(\mathrm{mol} \mathrm{m}^{-2} \mathrm{~s}^{-1}\right)$ & $6.6 \times 10^{-2}$ & Aeration rate \\
\hline$t_{0}(\mathrm{hr})$ & 48 & Operation time of each batch cycle \\
\hline
\end{tabular}

Table 6.3 Diffusion coefficients of all species

\begin{tabular}{|l|l|l|l|}
\hline Species & $\begin{array}{l}\text { in free solution } \\
\left(\times 10^{-9} \mathrm{~m}^{2} \mathrm{~s}^{-1}\right)\end{array}$ & $\begin{array}{l}\text { in CEM } \\
\left(\times 10^{-9} \mathrm{~m}^{2} \mathrm{~s}^{-1}\right)\end{array}$ & Comment \\
\hline $\mathrm{Na}^{+}$ & 1.33 & 0.1064 & \\
\hline $\mathrm{Cl}^{-}$ & 2.02 & 0.1056 & Ref. (Kim et al. \\
\hline $\mathrm{HAc}$ & 1.21 & 0.0067 & \\
\hline $\mathrm{Ac}-$ & 1.10 & 0.0110 & \\
\hline $\mathrm{NH}_{4}^{+}$ & 1.94 & 0.0795 & \\
\hline $\mathrm{NH}_{3}$ & 2.10 & 0.0210 & \\
\hline $\mathrm{H}_{2} \mathrm{CO}_{3}$ & 1.92 & 0.1536 & \\
\hline $\mathrm{HCO}_{3}^{-}$ & 1.18 & 0.0354 & \\
\hline $\mathrm{CO}_{3}^{2-}$ & 0.98 & 0.0294 & \\
\hline $\mathrm{H}^{+}$ & 9.13 & 0.3296 & \\
\hline $\mathrm{OH}^{-}$ & 5.16 & 0.1481 & \\
\hline
\end{tabular}

Table 6.4 Initial conditions in the anolyte and catholyte in numerical simulations

\begin{tabular}{|l|l|l|l|}
\hline Species & $\begin{array}{l}\text { Concentration in } \\
\text { anolyte }(\mathrm{mM})\end{array}$ & $\begin{array}{l}\text { Concentration in } \\
\text { catholyte }(\mathrm{mM})\end{array}$ & Comment \\
\hline $\mathrm{Na}^{+}$ & 45.199 & $1.000 \times 10^{-5}$ & $\begin{array}{l}10 \mathrm{mM} \text { in catholyte in the } \\
\text { parametric study }\end{array}$ \\
\hline $\mathrm{Cl}^{-}$ & 58.834 & $1.000 \times 10^{-4}$ & \\
\hline $\mathrm{HAc}$ & $2.597 \times 10^{-2}$ & $9.517 \times 10^{-7}$ & \\
\hline $\mathrm{Ac}-$ & 18.259 & $9.962 \times 10^{-5}$ & \\
\hline $\mathrm{NH}_{4}^{+}$ & 54.837 & $1.002 \times 10^{-4}$ & \\
\hline $\mathrm{NH}_{3}$ & 1.248 & $3.396 \times 10^{-7}$ & $\begin{array}{l}3.203 \mathrm{mM} \text { in catholyte in } \\
\text { parametric study }\end{array}$ \\
\hline $\mathrm{H}_{2} \mathrm{CO}_{3}$ & 1.264 & $6.077 \times 10^{-6}$ & $\begin{array}{l}9.993 \mathrm{mM} \text { in catholyte in } \\
\text { parametric study }\end{array}$ \\
\hline $\mathrm{HCO}_{3}^{-}$ & 22.856 & $1.635 \times 10^{-5}$ & $\begin{array}{l}3.264 \times 10^{-3} \mathrm{mM} \text { in catholyte in } \\
\text { parametric study }\end{array}$ \\
\hline $\mathrm{CO}_{3}{ }^{2-}$ & $4.326 \times 10^{-2}$ & $4.720 \times 10^{-9}$ & \\
\hline $\mathrm{H}^{+}$ & $2.471 \times 10^{-5}$ & $1.660 \times 10^{-4}$ & \\
\hline $\mathrm{OH}^{-}$ & $4.047 \times 10^{-4}$ & $6.024 \times 10^{-5}$ & \\
\hline
\end{tabular}

* The initial species concentration in catholyte is mostly practically 0 in the validation simulation, the same as in the experiment. In the parametric study, the initial species concentration is the same unless otherwise denoted. 


\subsubsection{Modeling of electrochemical and chemical reactions in the MEC}

Because of bacteria activity, acetate $\left(\mathrm{Ac}^{-}\right)$is oxidized on the anode to release electron and proton:

$$
\mathrm{Ac}^{-}+4 \mathrm{H}_{2} \mathrm{O} \rightarrow 2 \mathrm{HCO}_{3}^{-}+9 \mathrm{H}^{+}+8 \mathrm{e}^{-}
$$

(Equation 6.7)

Through the external circuit, the released electrons are transferred to the cathode and react with the oxygen from the aeration:

$$
4 \mathrm{e}^{-}+\mathrm{O}_{2}+2 \mathrm{H}_{2} \mathrm{O} \rightarrow 4 \mathrm{OH}^{-}
$$

Since both equations are directly related to the electron transfer, the consumption/production of related species within the system can be modeled as a function of current, and they are referred to as Faradaic consumption/production in the main text.

The concentration of a species in the anolyte/catholyte/membrane can change due to its transport, Faradaic reactions (if applicable) and acid-base type chemical reactions. The effects of the acidbase type reactions are modeled as a sink/source term. Here, we illustrate the treatment of these acid-base type reactions using the evolution of $\mathrm{NH}_{4}{ }^{+} / \mathrm{NH}_{3}$ concentration inside the anolyte as an example. For this couple, their reaction $\mathrm{NH}_{3}+\mathrm{H}^{+} \leftrightarrows \mathrm{NH}_{4}^{+}$and their transport flux collectively change their concentrations. In the anolyte, the evolution of their concentrations is governed by

$$
\begin{gathered}
V_{1} \frac{\partial C_{\mathrm{NH}_{4}^{+}}^{1}}{\partial t}=A \cdot J_{\mathrm{NH}_{4}^{+}, 1}^{\prime}+V_{1} \dot{S}_{\mathrm{NH}_{3} \rightarrow \mathrm{NH}_{4}^{+}}^{c} \\
V_{1} \frac{\partial C_{\mathrm{NH}_{3}}^{1}}{\partial t}=A \cdot J_{\mathrm{NH}_{3}, 1}^{\prime}-V_{1} J_{\mathrm{NH}_{3}, 1}^{c}
\end{gathered}
$$

where $J_{\mathrm{NH}_{4}^{+}, 1}^{\prime}$ and $J_{\mathrm{NH}_{3}, 1}^{\prime}$ are transport of $\mathrm{NH}_{4}{ }^{+}$and $\mathrm{NH}_{3}$ into the anolyte through the CEM, respectively. $V_{1} \dot{S}_{\mathrm{NH}_{3} \rightarrow \mathrm{NH}_{4}^{+}}$represents the amount of $\mathrm{NH}_{3}$ converted to $\mathrm{NH}_{4}^{+}$due to the reaction 
$\mathrm{NH}_{3}+\mathrm{H}^{+} \leftrightarrows \mathrm{NH}_{4}^{+}$. Combing Equation 6.9 and 6.10 gives a new equation lumping the $\mathrm{NH}_{4}{ }^{+}$and $\mathrm{NH}_{3}$ together.

$$
V_{1} \frac{\partial C_{\mathrm{NH}_{4}^{+}}^{1}}{\partial t}+V_{1} \frac{\partial C_{\mathrm{NH}_{3}}^{1}}{\partial t}=A \cdot\left(J_{\mathrm{NH}_{4}^{+}, 1}^{\prime}+J_{\mathrm{NH}_{3}, 1}^{\prime}\right)
$$

Assuming fast equilibrium for the acid-base reaction, the partition between the $\mathrm{NH}_{4}{ }^{+}$and $\mathrm{NH}_{3}$, follows (Dykstra et al. 2014b, Kuntke et al. 2012, Persat et al. 2009, Powers et al. 1987):

$$
K_{\mathrm{a}, \mathrm{NH}}=\frac{C_{\mathrm{NH} 3}^{1} \cdot C_{\mathrm{H}^{+}}^{1}}{C_{\mathrm{NH}_{4}^{+}}^{1}}
$$

where $K_{\mathrm{a}, \mathrm{NH}}$ is the chemical equilibrium constant for the reaction $\mathrm{NH}_{3}+\mathrm{H}^{+} \leftrightarrows \mathrm{NH}_{4}^{+}$. Equation 15 states that the ratio of the $\mathrm{NH}_{4}{ }^{+}$and $\mathrm{NH}_{3}$ concentrations are directly related to the $\mathrm{pH}$ locally. As shown in Figure 6.3, for $\mathrm{pH}<7 \mathrm{NH}_{4}{ }^{+}$counts more than $99.4 \%$ of the total ammonium/ammonia; for $\mathrm{pH}>9.5, \mathrm{NH}_{3}$ counts more than $64 \%$. Similar mathematical treatment is applied to all other acid-base type reactions. A full list of the acid-base reactions and their equilibrium constant is provided in Table 6.2 .

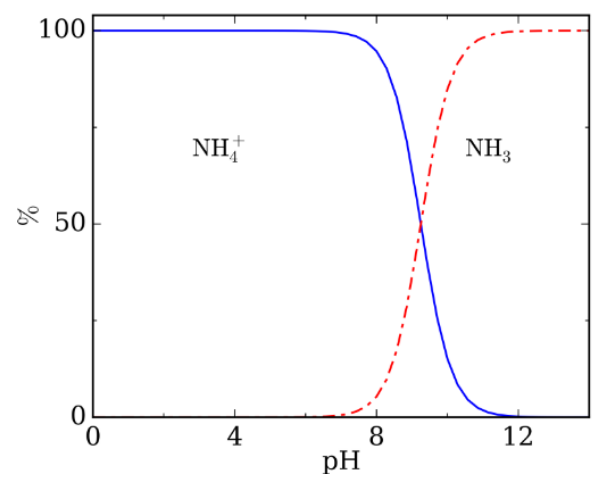

Figure 6.3 Partition between $\mathrm{NH}_{4}^{+}$and $\mathrm{NH}_{3}$ as a function of $\mathrm{pH}$ assuming fast $\mathrm{NH}_{3}+\mathrm{H}^{+}=\mathrm{NH}_{4}{ }^{+}$ reaction.

\subsubsection{Governing equation for $\mathrm{H}^{+}$ion concentration in the anolyte}

Equation 6.14 shows how the $\mathrm{H}^{+}$ion concentration in the anolyte evolves a function of time. Here we elaborate on the meaning of each term in the equation and how it is evaluated computationally. 
We note that there are several possible ways to change the $\mathrm{H}^{+}$ion concentration in anolyte: the flux of proton into/out of the anode chamber, the Faradaic reaction due to the bacteria oxidation, and the consumption/production due to reaction with other species, which include all the acid-base reactions shown in Table 6.1. Using conservation law, Equation 6.14 is obtained as (reproduced here for convenience)

$$
V_{1} \frac{\partial C_{\mathrm{H}^{+}}^{1}}{\partial t}=A \cdot J_{H^{+}, 1}^{\prime}+\frac{9}{8} \frac{I A}{F}-V_{1} \dot{S}_{\mathrm{NH}_{3} \rightarrow \mathrm{NH}_{4}^{+}}-V_{1} \dot{S}_{\mathrm{Ac}^{-} \rightarrow \mathrm{HAc}}-V_{1} \dot{S}_{\mathrm{OH}^{-} \rightarrow \mathrm{H}_{2} \mathrm{O}}-V_{1} \dot{S}_{\mathrm{HCO}_{3}^{-} \rightarrow \mathrm{H}_{2} \mathrm{CO}_{3}}+V_{1} \dot{S}_{\mathrm{HCO}_{3}^{-} \rightarrow \mathrm{CO}_{3}^{2-}}
$$

(Equation 6.13)

As explained in Equs. $\mathrm{S} 3$ and $\mathrm{S} 4, V_{1} \dot{S}_{\mathrm{NH}_{3} \rightarrow \mathrm{NH}_{4}^{+}}$stands for the conversion of $\mathrm{NH}_{3}$ to $\mathrm{NH}_{4}^{+}$by the reaction $\mathrm{NH}_{3}+\mathrm{H}^{+} \rightarrow \mathrm{NH}_{4}^{+}$, which equals to the consumption of proton. This term can be calculated by manipulating Equation 6.10, giving $V_{1} \dot{S}_{\mathrm{NH}_{3} \rightarrow \mathrm{NH}_{4}^{+}}=-\left(V_{1} \frac{\partial C_{\mathrm{NH}_{3}}^{1}}{\partial t}-A \cdot J_{\mathrm{NH}_{3}, 1}^{\prime}\right)$; Using the $J_{\mathrm{NH}_{3}, 1}^{\prime}$ calculated in simulations, $V_{1} \dot{S}_{\mathrm{NH}_{3} \rightarrow \mathrm{NH}_{4}^{+}}$can be evaluated. Similar process could be applied to all other source/sink terms in Equation 6.13 in the main text and the results are as follows: $\quad V_{1} \dot{S}_{\mathrm{Ac}^{-} \rightarrow \mathrm{HAc}}=V_{1} \frac{\partial C_{\mathrm{HAc}}^{1}}{\partial t}-A \cdot J_{\mathrm{HAc}, 1}^{\prime} \quad, \quad V_{1} \dot{S}_{\mathrm{HCO}_{3}^{-} \rightarrow \mathrm{H}_{2} \mathrm{CO}_{3}}=V_{1} \frac{\partial C_{\mathrm{H}_{2} \mathrm{CO}_{3}}^{1}}{\partial t}-A \cdot J_{\mathrm{H}_{2} \mathrm{CO}_{3}, 1}^{\prime}$, $V_{1} \dot{S}_{\mathrm{HCO}_{3}^{-} \rightarrow \mathrm{CO}_{3}^{2-}}=V_{1} \frac{\partial C_{\mathrm{CO}_{3}^{2-}}^{1}}{\partial t}-A \cdot J_{\mathrm{CO}_{3}^{2-}, 1}^{\prime}, V_{1} \dot{S}_{\mathrm{OH}^{-} \rightarrow \mathrm{H}_{2} \mathrm{O}}=-\left(V_{1} \frac{\partial C_{\mathrm{OH}^{-}}^{1}}{\partial t}-A \cdot J_{\mathrm{OH}^{-}, 1}^{\prime}\right)$. All variables are named under the same convention as in the main text, i.e., $C_{i}^{1}$ stands for the concentration of species $i$ in the anolyte and $J_{i, j}^{\prime}$ is the flux of species $i$ into chamber $j(j=1$ : anode chamber; $j=2$ cathode chamber).

\subsection{Results}

A bench-scale cubic shape MEC was used for experimental data collection (see Figure 6.1). The wastewater fed into the anode was synthetic digestion effluent of livestock waste while deionized water was used as catholyte. The anolyte was partially replaced $(150 \mathrm{~mL})$ every $48 \mathrm{~h}$ while the 
catholyte was unchanged during 3 batch cycles. The variation of organic matters, $\mathrm{NH}_{4}^{+} / \mathrm{NH}_{3}$ concentration, $\mathrm{pH}$ and inert ions in both chambers were recorded for analysis and model validation.

To simulate the batch-mode operation of MECs for ammonia recovery, we extend the steady-state models developed earlier (Dykstra et al. 2014c). The models consider the mass conservation for all species (without loss of generality, the following species are included: $\mathrm{Na}^{+}, \mathrm{Cl}^{-}, \mathrm{HAc}, \mathrm{Ac}^{-}$, $\mathrm{NH}_{4}^{+}, \mathrm{NH}_{3}, \mathrm{H}_{2} \mathrm{CO}_{3}, \mathrm{HCO}_{3}^{-}, \mathrm{CO}_{3}{ }^{2-}, \mathrm{H}^{+}$and $\mathrm{OH}^{-}$), the transport of each species (diffusion and migration) across the membrane, the chemical reactions among different species, and gas-solution equilibrium. For chemical reactions, we consider mostly acid-base reactions essential for ammonia recovery (e.g., $\mathrm{NH}_{3}+\mathrm{H}^{+} \rightleftharpoons \mathrm{NH}_{4}^{+}, \mathrm{H}_{2} \mathrm{CO}_{3} \rightleftharpoons \mathrm{H}^{+}+\mathrm{HCO}_{3}^{-}, \ldots$ and a full list of these reactions is provided in the Supplementary Information). Using the operation parameters (e.g., the anolyte/catholyte volume $\mathrm{V}_{1} / \mathrm{V}_{2}$, the membrane surface area $A$, etc.) and the measured current density as input, the model can predict the time evolution of the concentration of each species in the system (hereafter, the concentration of species $i$ in anolyte/catholyte chamber is denoted as $c_{i}^{j}$, with $j=1$ and 2 for anode and cathode chamber, respectively) and the recovery of ammonia by aeration. Below we first validate the mathematical model, then investigate the above issues and how they impact the ammonia recovery in the MEC.

\subsubsection{Model validation}

We first validate the mathematical model for ammonia recovery using MEC by comparing its predictions against experimental data obtained under the same operation conditions. Ammonium recovery was firstly studied experimentally. During one batch cycle, the COD concentration decreased from $1089 \pm 169$ to $262 \pm 83 \mathrm{mg} \mathrm{L}^{-1}$, resulting in a Coulombic efficiency (CE) of 34.7 
$\pm 5.9 \%$. The maximum current density was $1.89 \mathrm{~A} \mathrm{~m}^{-2}$ (Figure 6.2a). In the anolyte, the $\mathrm{pH}$ decreased from $7.98 \pm 0.08$ to $4.52 \pm 0.63$; in the catholyte, the $\mathrm{pH}$ increased dramatically initially and then stabilized at $\sim 9.7$ (Figure $6.2 \mathrm{~b}$ ). The acetate concentration in the anolyte decreased from $15.6 \pm 2.4$ to $5.4 \pm 0.9 \mathrm{mM}$, and that in the catholyte was always below the detection limit of our equipment (Figure 6.2c). The latter is consistent with the fact that the diffusion loss of acetate through CMI-7000 is small (Kim et al. 2007b). Experimentally, the total input nitrogen as ammonium in each batch cycle was $9.3 \pm 0.4 \mathrm{mmol}$, among which $35.7 \pm 7.4 \%$ stayed in the anolyte, $0.9 \pm 0.3 \%$ remained in the catholyte and $66.2 \pm 2.7 \%$ was stripped out as ammonia and absorbed by the sulfuric acid. In each batch cycle (2 days), $6.1 \pm 0.1 \mathrm{mmol}$ of $\mathrm{NH}_{3}$ gas was collected through aeration, resulting in a recovery rate of $10.2 \pm 0.1 \mathrm{gN} \mathrm{m}^{-2} \mathrm{~d}^{-1}$ (vs. membrane surface area) (CordRuwisch et al. 2011). Ammonia oxidation in the anode chamber might not occur because of we did not detect any nitrite or nitrate in the anolyte. Numerically, the simulation predicted the total ammonia concentration in anolyte and catholyte to be $5 \%$ and $18.4 \%$ within the corresponding maximum measured values, respectively. Some $\mathrm{Na}^{+}$and $\mathrm{Cl}^{-}$ions crossed into the catholyte through the CEM in each cycle. To assess their role in the net charge transport through the CEM, we computed the cumulative transport number $\bar{t}_{i}^{+}=V_{1} z_{i} F\left(c_{i}^{1}(0)-c_{i}^{1}(t)\right) / \int_{0}^{t} I A d t$ for these ions during each cycle. At the end of each cycle, $\bar{t}_{\mathrm{Na}^{+}}^{+}=0.124 \pm 0.044$ and $\bar{t}_{\mathrm{Cl}}^{+}=-0.021 \pm 0.004$ (Figure 6.2f), indicating that overall the transport of these ions contributes to $\sim 10 \%$ of the total current. Since the transport of all other ions except $\mathrm{NH}_{4}^{+}$ions through the CEM is very small, we thus conclude that the transport of $\mathrm{NH}_{4}^{+}$ions through the CEM accounts for $\sim 90 \%$ of the total current in our system, which is consistent with some earlier studies (Cord-Ruwisch et al. 2011). 
The above ammonia recovery operation was also simulated using the mathematical model developed. As shown in Figure 6.2b to Figure 6.2f, the model predictions agree quite well with the experimental data. In particular, the model captures the evolution of $\mathrm{pH}$, acetate concentration, and total nitrogen content in the anolyte/catholyte. The recovery of $\mathrm{NH}_{3}$ gas was also accurately captured. The model underestimates the transport of $\mathrm{Na}^{+}$ions across the CEM (Figure 6.2f). However, given that the transport of $\mathrm{Na}^{+}$ions accounts for only a small fraction of the total current through the CEM and these ions do not interact with other species in the system, these small deviations are deemed acceptable.

Using the validated model, we next examine the mechanisms of MEC-based ammonia recovery using simulations and address three issues outlined earlier, i.e., the $\mathrm{pH}$ regulation, $\mathrm{NH}_{4}{ }^{+}$transport and ion competition in MECs. The initial conditions in the anolyte are the same as those in the above experiment. The catholyte initially features $10 \mathrm{mM}$ of $\mathrm{Na}^{+}$ions and accompanying carbonate group ions, which together produces a $\mathrm{pH}$ of $\sim 6.8$, as commonly found in experiments. Without a loss of generality, the system operates at a fixed current density of $\mathrm{I}=1 \mathrm{~A} \mathrm{~m}^{-2}$ for 48 hours.

\subsection{2 $\mathrm{pH}$ regulation}

To understand how the $\mathrm{pH}$ is regulated, we note that, by considering the mass conservation of proton, its concentration evolution in the anolyte follows:

$$
V_{1} \frac{\partial C_{\mathrm{H}^{+}}^{1}}{\partial t}=A \cdot J_{H^{+}, 1}^{\prime}+\frac{9 I A}{8} \frac{I A}{F}-V_{1} \dot{S}_{\mathrm{NH}_{3} \rightarrow \mathrm{NH}_{4}^{+}}-V_{1} \dot{S}_{\mathrm{Ac}^{-} \rightarrow \mathrm{HAc}}-V_{1} \dot{S}_{\mathrm{OH}^{-} \rightarrow \mathrm{H}_{2} \mathrm{O}}-V_{1} \dot{S}_{\mathrm{HCO}_{3}^{-} \rightarrow \mathrm{H}_{2} \mathrm{CO}_{3}}+V_{1} \dot{S}_{\mathrm{HCO}_{3}^{-} \rightarrow \mathrm{CO}_{3}^{2-}}
$$

(Equation 6.14) This equation shows that the proton concentration in the anolyte is affected by several factors: the transport of proton into or out of the anolyte through the CEM (the first term on the right side), the 
production of proton through Faraday reactions (the second term on right), and the consumption or production of protons by various chemical reactions (the remaining terms on right), e.g., $V_{1} \dot{S}_{\mathrm{NH}_{3} \rightarrow \mathrm{NH}_{4}^{+}}$stands for the consumption of proton by the reaction $\mathrm{NH}_{3}+\mathrm{H}^{+} \rightarrow \mathrm{NH}_{4}^{+}$. For brevity, the last two terms in Equation 6.13 are hereafter lumped as $\dot{S}_{\text {carb }}$.
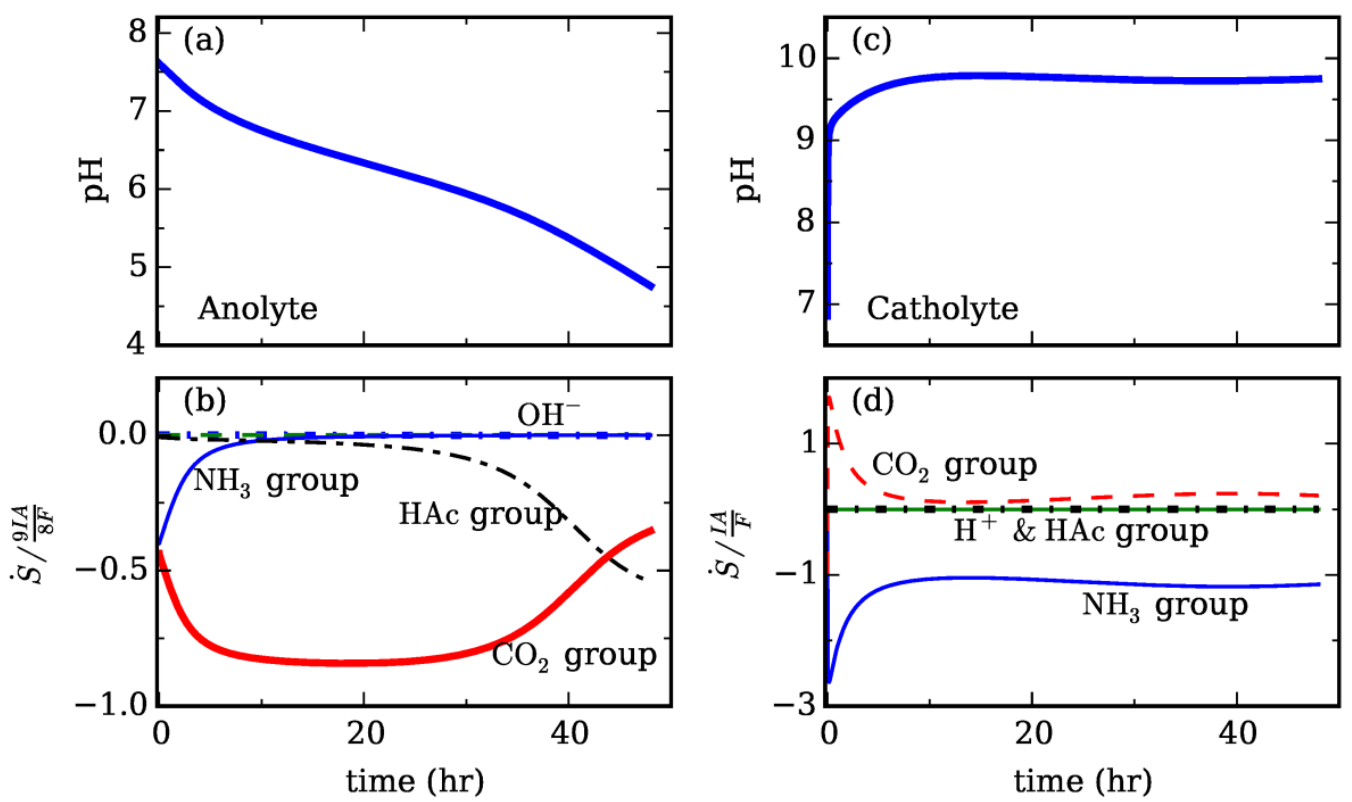

Figure 6.4 Regulation of $\mathrm{pH}$ in $\mathrm{MEC}$ during ammonia recovery operation. The variation of the $\mathrm{pH}$ values in the anolyte (a) and the catholyte (c) is governed by various Faradaic and chemical reactions (b and d) identified in Equation 6.11-6.15. The generation/consumption of proton and hydroxide by the chemical reactions involving various ion groups in the anolyte and catholyte are shown in (b) and (d), respectively. These generation/consumption terms are normalized by the production of proton (in anolyte, for panel b) and hydroxide (in catholyte, for panel d) generated through Faradic reactions. [Operating conditions and parameters in this study are the same as in Figure 6.2 except the followings: the current is $I=1 \mathrm{~A} / \mathrm{m}^{2}$; the catholyte initially contains $10 \mathrm{mM}$ of $\mathrm{Na}^{+}$ions and the accompanying carbonate group ions to produce a $\mathrm{pH}$ of $\sim 6.8$ ].

To understand how the $\mathrm{pH}$ in the anolyte is regulated, we simulated the operation of MEC under a constant current of $\mathrm{I}=1 \mathrm{~A} \mathrm{~m}^{-2}$. Figure $6.4 \mathrm{a}$ shows that the $\mathrm{pH}$ in the anolyte decreases steadily during the operation. To assess the relative importance of the various factors identified above in regulating the $\mathrm{pH}$, we examined each term on the right-hand side of Equation 6.13 and the results are shown in Figure 6.4b (all terms are normalized by the Faradaic reaction term). We observe that 
most of the protons generated by the Faradaic reaction were neutralized by their reactions with $\mathrm{NH}_{3}$, the $\mathrm{Ac}^{-}$ions, and the carbonate ions. Hence, these chemical groups essentially serve as the "buffer" to keep the $\mathrm{pH}$ from changing dramatically. The relative contribution of these chemical groups in regulating the $\mathrm{pH}$ evolves during the operation of MEC: $\mathrm{NH}_{3}$ lost its buffer capability soon after the $\mathrm{pH}$ is below 7, while the carbonate ions remain effective in buffering the anolyte till $\sim 40 \mathrm{hrs}$.

A similar equation for the evolution of the hydroxide concentration in the catholyte was derived to understand the regulation of $\mathrm{pH}$ therein:

$V_{2} \frac{\partial C_{\mathrm{OH}^{-}}^{2}}{\partial t}=A J_{\mathrm{OH}^{-}, 2}^{\prime}+\frac{I A}{F}-V_{1} \dot{S}_{\mathrm{HAc} \rightarrow \mathrm{Ac}^{-}}-V_{1} \dot{S}_{\mathrm{NH}_{4}^{+} \rightarrow \mathrm{NH}_{3}}-V_{1} \dot{S}_{\mathrm{H}^{+} \rightarrow \mathrm{H}_{2} \mathrm{O}}-V_{1} \dot{S}_{\mathrm{H}_{2} \mathrm{CO}_{3} \rightarrow \mathrm{HCO}_{3}^{-}}-V_{1} \dot{S}_{\mathrm{HCO}_{3}^{-} \rightarrow \mathrm{CO}_{3}^{2-}}$

(Equation 6.15) This equation shows that the hydroxide concentration in the catholyte is affected by the transport of hydroxide into/out of the catholyte (the first term on the right side), the production of hydroxide by Faradaic reactions (the second term on right), and various chemical reactions consuming or producing hydroxide (the remaining terms on right). The last two terms are hereafter lumped as $\dot{S}_{\text {carb }}$. Figure 6.4c-d show the $\mathrm{pH}$ variation and the relative magnitude of each reaction term on the right-hand side of Equation 6.15. We observe that, in the catholyte, the $\mathrm{pH}$ was predominately controlled by the Faradaic production of hydroxide and the consumption of hydroxide by their reaction with the $\mathrm{NH}_{4}{ }^{+}$ions. Since there are few $\mathrm{NH}_{4}{ }^{+}$ions inside the catholyte at the very beginning, the $\mathrm{pH}$ value rises rapidly during the earliest stage of operation. Unlike the $\mathrm{NH}_{4}{ }^{+}$ions, the carbonate ions tend to increase the $\mathrm{pH}$ value because their chemical reactions inside the catholyte consume proton. Overall, the effect of carbonate ions on the $\mathrm{pH}$ in catholyte is limited, and this is related to their low concentration inside the catholyte. The latter is due to the limited 
carbonate ions inside the anolyte and their slow diffusion through the CEM. Since the $\mathrm{pH}$ regulation inside the catholyte depends strongly on the availability of $\mathrm{NH}_{4}{ }^{+}$ions, and the latter in turn depends on the transport of $\mathrm{NH}_{4}{ }^{+}$ions and $\mathrm{NH}_{3}$ across the CEM, it is clear that the $\mathrm{pH}$ regulation is intimately coupled with the transport of these species.

\subsubsection{Ammonium and ammonia transport}

Such transport is analyzed from the perspective of how these species are removed from the anolyte. There are three ways to remove $\mathrm{NH}_{3} / \mathrm{NH}_{4}{ }^{+}$ion from the anolyte: diffusion and migration of $\mathrm{NH}_{4}{ }^{+}$ ion through the CEM and the diffusion of $\mathrm{NH}_{3}$ through CEM. Figure 6.5a shows that, under the moderate electrical current considered here $\left(\mathrm{I}=1 \mathrm{~A} \mathrm{~m}^{-2}\right)$, the diffusion of $\mathrm{NH}_{4}{ }^{+}$is actually stronger than its electrical migration. This somewhat surprising result is caused largely by the different $\mathrm{pH}$ value inside the anolyte and catholyte. As shown in Figure 6.2, the anolyte (catholyte) is strongly acidic (basic) during operation. Hence, nitrogen element exists mostly as $\mathrm{NH}_{4}{ }^{+}$in the anolyte and as $\mathrm{NH}_{3}$ in the catholyte (Figure 6.3). Consequently, the $\mathrm{NH}_{4}{ }^{+}$concentration in the anolyte is always much higher than that in the catholyte (Figure 6.5b), which leads to a strong diffusion flux of the $\mathrm{NH}_{4}{ }^{+}$ions. Since the acidic (basic) $\mathrm{pH}$ in the anolyte (catholyte) is ultimately induced by the electrical current through the system, we see that the imposed current serves two roles: it helps drive the migration of $\mathrm{NH}_{4}{ }^{+}$ions from the anolyte toward catholyte, and it helps set up the $\mathrm{pH}$ environment that favors the diffusion of $\mathrm{NH}_{4}{ }^{+}$ion from anolyte chamber toward the catholyte

chamber. The second role has not been widely recognized. However, the fact that the diffusion of $\mathrm{NH}_{4}{ }^{+}$ions dominates over their migration suggests that this second role is at least as important as the first role. We note that, while diffusion dominates the transport of $\mathrm{NH}_{4}{ }^{+}$under low/moderate current densities (e.g., the $\mathrm{I}=1 \mathrm{~A} \mathrm{~m}^{-2}$ considered here), electrical migration can become the 
dominant transport mechanism at high current densities (e.g., at $\mathrm{I}>5 \mathrm{~A} \mathrm{~m}^{-2}$ ) (Desloover et al. 2012).

It is worth pointing out that, in absence of a net current, because of the low cation concentration in our catholyte (hence limited cation exchange between the CEM and catholyte), the removal of $\mathrm{NH}_{4}{ }^{+}$from anolyte through transport across the CEM is minor. Indeed, both simulations and experiments suggest that $\sim 2 \%$ of $\mathrm{NH}_{4}{ }^{+}$could be removed from the anolyte in one batch cycle (48 hours), which is consistent with some prior studies (Zhang et al. 2014a).

The dominance of $\mathrm{NH}_{3}$ over $\mathrm{NH}_{4}{ }^{+}$ions inside the catholyte and the opposite trend in the anolyte implies that there can exist strong diffusion of $\mathrm{NH}_{3}$ from the catholyte chamber toward the anolyte chamber. Such diffusion can potentially lower the recovery efficiency of $\mathrm{NH}_{3}$ from the catholyte (Dykstra et al. 2014c). However, Figure 6.5d shows that, although there is a relatively large amount of $\mathrm{NH}_{3}$ diffuse toward the anolyte chamber at the CEM-catholyte interface, very little $\mathrm{NH}_{3}$ crosses CEM-anolyte interface into the anolyte. This interesting phenomenon is caused by the $\mathrm{pH}$ environment within the CEM. As shown in Figure 6.5d, within the CEM, the $\mathrm{pH}$ is strongly acidic at the anolyte side, which favors conversion of $\mathrm{NH}_{3}$ to $\mathrm{NH}_{4}^{+}$ions by $\mathrm{NH}_{3}+\mathrm{H}^{+} \rightarrow \mathrm{NH}_{4}^{+}$reaction. Hence, the anode side of the membrane served as the "reactor": the $\mathrm{NH}_{3}$ diffused toward the anolyte chamber was turned into $\mathrm{NH}_{4}{ }^{+}$within the membrane and subsequently diffused back into the cathode chamber. 

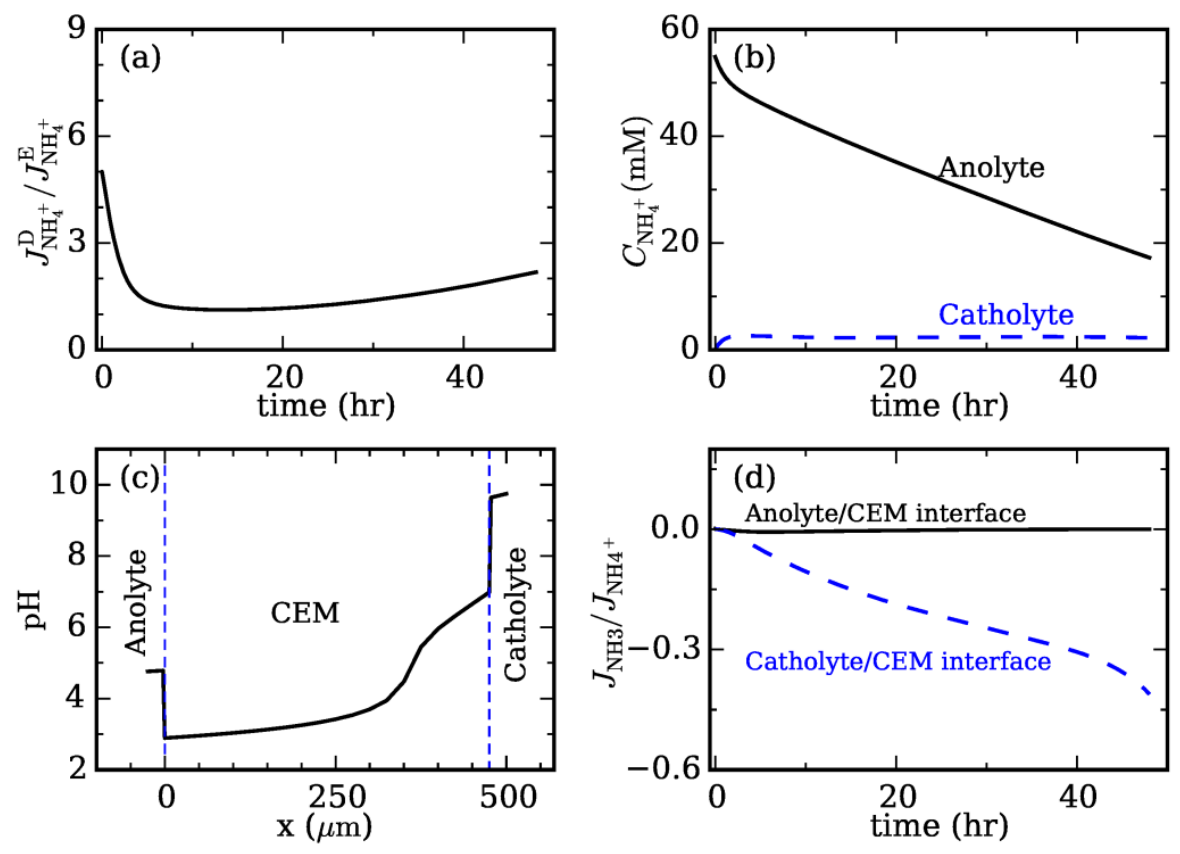

Figure 6.5 Mechanisms of $\mathrm{NH}_{4}^{+} / \mathrm{NH}_{3}$ transport in MECs. (a) Relative strength of $\mathrm{NH}_{4}{ }^{+}$ion transport due to diffusion and migration. (b) Evolution of the $\mathrm{NH}_{4}{ }^{+}$ion concentration in the anolyte and catholyte. (c) Distribution of $\mathrm{pH}$ across the CEM and at the CEM-anolyte/catholyte interfaces. (d) The diffusion flux of $\mathrm{NH}_{3}$ toward the anolyte at the CEM-catholyte/anolyte interfaces. These diffusion fluxes are normalized by the total $\mathrm{NH}_{4}{ }^{+}$ion flux to highlight their impact on the ammonia recovery in the catholyte. [ALL parameters in this study are the same as in Fig. 3.]

More generally, the relevance of $\mathrm{NH}_{3}$ diffusion from catholyte to anolyte to its recovery can be estimated. The diffusion of $\mathrm{NH}_{3}$ is $J_{\mathrm{NH}_{3}} \sim D_{\mathrm{NH}_{3}}^{m} C_{\mathrm{NH}_{3}}^{2} / L\left(D_{\mathrm{NH}_{3}}^{m}\right.$ : diffusion coefficient of $\mathrm{NH}_{3}$ in the membrane; $C_{\mathrm{NH}_{3}}^{2}: \mathrm{NH}_{3}$ concentration in the catholyte; $L$ : membrane thickness). The evaporating flux of $\mathrm{NH}_{3}$ in the catholyte is $J_{\mathrm{NH}_{3}}^{\text {evap }}=C_{\mathrm{NH}_{3}}^{2} Q_{\text {inert }} / A p_{\text {tot }} K_{\mathrm{H}, \mathrm{NH}}$ (Dykstra et al. 2014c, Powers et al. 1987). Hence, we define a dimensionless number

$$
\Pi=\frac{D_{N H_{3}}^{m} p_{t o t} K_{H, N H} A}{L Q_{\text {inert }}}
$$

(Equation 6.16)

When $\Pi \ll 1$, the effect of diffusion on ammonia recovery is negligible. For the system studied here, we have $D_{\mathrm{NH}_{3}}^{\mathrm{m}}=2.1 \times 10^{-11} \mathrm{~m}^{2} / \mathrm{s}, p_{\text {tot }}=1 \mathrm{bar}, K_{\mathrm{H}, \mathrm{NH}}=56250 \mathrm{mM} / \mathrm{bar}, \mathrm{L}=475 \mu \mathrm{m}($ Aeshala et al. 2013), and aeration rate $Q_{\text {inert }} / A=0.066 \mathrm{~mol} /\left(\mathrm{m}^{2} \mathrm{~s}\right)$. Hence, $\Pi=0.0376$, i.e., the diffusion is 
weak enough to be ignored, which is consistent with the result in Figure 6.5c. Since $\Pi$ depends only on the design and operational parameters of the MEC, it can be used conveniently during the design of MECs.

\subsubsection{Ion competition}

For a fixed current, various cations (e.g., $\mathrm{Na}^{+}$) in the anolyte can compete with the $\mathrm{NH}_{4}{ }^{+}$ions for transport across the CEM, thus potentially compromising the removal of $\mathrm{NH}_{4}{ }^{+}$ions from the anolyte. To quantitatively examine this competition, we computed the transport number of $\mathrm{NH}_{4}{ }^{+}$ and $\mathrm{Na}^{+}$ions at the CEM-anolyte interface, i.e., $t_{i}^{+}=z_{i} F J_{i, 1}^{\prime} / I$ (Figure 6.6a). At short time $(t<$ 9hr), the transport number of the $\mathrm{Na}^{+}$ions is negative while that of the $\mathrm{NH}_{4}{ }^{+}$ions is larger than 1.0, i.e., $\mathrm{Na}^{+}$ions transport from the catholyte into the anolyte, and instead of competing with the $\mathrm{NH}_{4}{ }^{+}$ ions, they facilitate the removal of $\mathrm{NH}_{4}{ }^{+}$ions from the anolyte. This phenomenon weakens with increasing time. At $t>\sim 9 \mathrm{hr}, \mathrm{Na}^{+}$ions do compete with $\mathrm{NH}_{4}{ }^{+}$ions for transport across CEM, but the effect is still weak since the transport number of $\mathrm{NH}_{4}{ }^{+}$ions is $\sim 0.8$ even at $t=40 \mathrm{hr}$.

The above surprising results are caused largely by the Donnan effects. Driven by the Donnan equilibrium, the concentrations of cations at membrane-anolyte/catholyte interfaces are elevated greatly above their concentrations in the adjacent anolyte/catholyte to balance the negative fixed charge in the CEM. For $\mathrm{Na}^{+}$ions, their concentration at the CEM-catholyte interface is very high because there are few other cations in the catholyte competing with them to balance the fixed negative charge in the CEM (Figure 6.6b). Meanwhile, the $\mathrm{Na}^{+}$ions concentration at the CEManolyte interface is only moderately high because there are many $\mathrm{NH}_{4}{ }^{+}$ions in the anolyte to cooperate with $\mathrm{Na}^{+}$ions to balance CEM's fixed charge. Consequently, the concentration of $\mathrm{Na}^{+}$ 
ions within the CEM decreases from the catholyte side toward the anolyte, despite that there are more $\mathrm{Na}^{+}$ions in the anolyte than in catholyte. At short time, such a negative concentration gradient drives a strong diffusion flux, leading to the increase of the $\mathrm{Na}^{+}$ion concentration in the anolyte. Because of current conservation, more $\mathrm{NH}_{4}{ }^{+}$ions must be removed from the anolyte than that corresponding to the current passing out of the anolyte, thus leading to a facilitated $\mathrm{NH}_{4}{ }^{+}$ion transport, i.e., $\bar{t}_{\mathrm{NH}_{4}^{+}}^{+}>1$. At longer time, the $\mathrm{Na}^{+}$concentration at the CEM-anolyte interface increases (due to the decrease of $\mathrm{NH}_{4}{ }^{+}$concentration in the anolyte) and the opposite happens at the CEM-catholyte interface. Hence, the diffusion of $\mathrm{Na}^{+}$ions is weakened. Eventually, the diffusion of $\mathrm{Na}^{+}$ions becomes weaker than their migration toward catholyte (Figure 6.6b). However, since the migration is always partly canceled by the diffusion, $\mathrm{Na}^{+}$ions always compete weakly with $\mathrm{NH}_{4}{ }^{+}$ions for transport across the CEM. As a result, the current through the system is mostly carried by the $\mathrm{NH}_{4}^{+}$ions, which is consistent with our experimental data.

The above trends in ion competition are weakly modified when the initial concentration of $\mathrm{Na}^{+}$ ions in the anolyte changes. For higher initial concentration of $\mathrm{Na}^{+}$ions, the diffusion of $\mathrm{Na}^{+}$ions from catholyte to anolyte and its positive effect on $\mathrm{NH}_{4}{ }^{+}$ions removal from anolyte during the 
early stage of operation decreases moderately, and the competition of $\mathrm{Na}^{+}$ions for transport across CEM sets in earlier; for lower initial concentration of $\mathrm{Na}^{+}$ions, the opposite occurs.
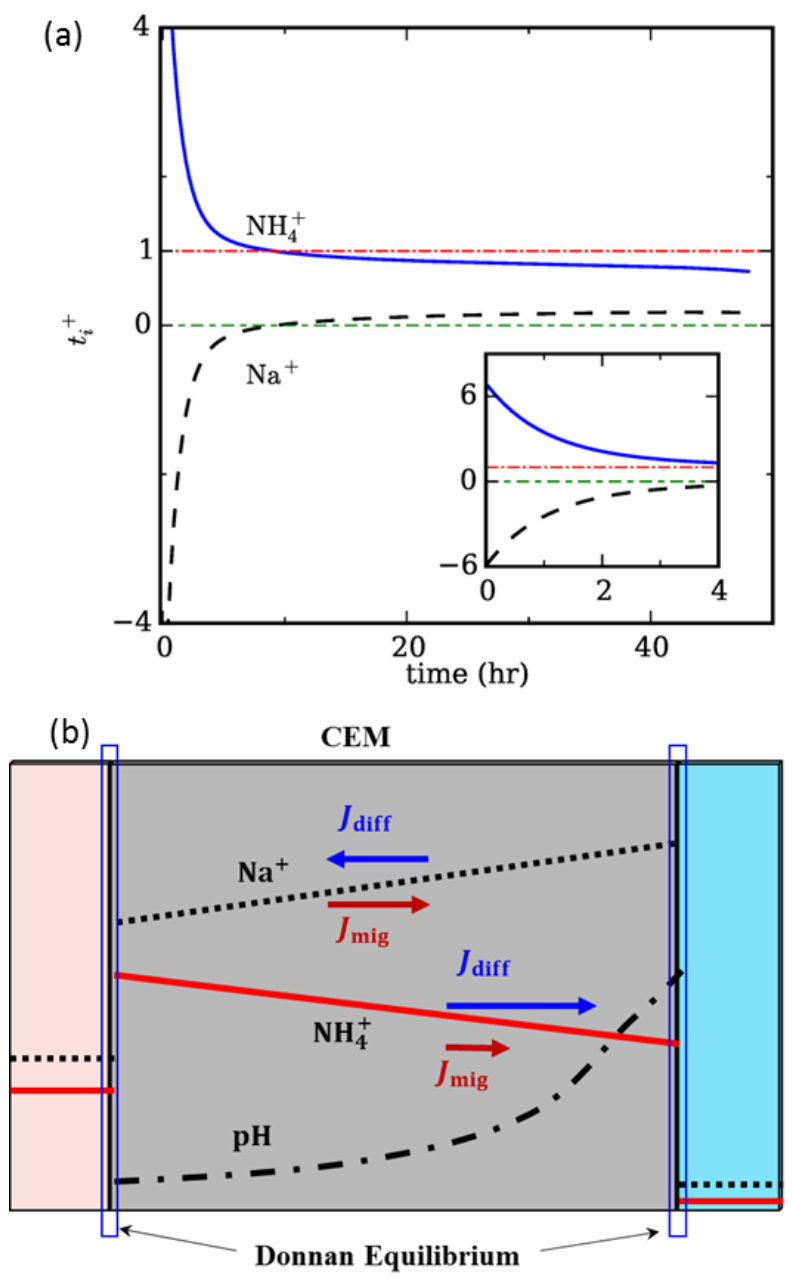

Figure 6.6 Ionic competition between $\mathrm{Na}^{+}$and $\mathrm{NH}_{4}^{+}$. (a) Transport number of $\mathrm{Na}^{+}$and $\mathrm{NH}_{4}{ }^{+}$ions at the CEM-anolyte interface. (b) The concentration profiles and transport of $\mathrm{Na}^{+}$and $\mathrm{NH}_{4}+$ ions within the cation exchange membrane. [ALL parameters in this study are the same as in Chapter 5]

Due to the opposite direction of their diffusion and migration through the CEM, $\mathrm{Na}^{+}$carries little charge across the CEM and the ion competition effect between $\mathrm{Na}^{+}$and $\mathrm{NH}_{4}{ }^{+}$is moderate. Here we assess the ion competition effect when the $\mathrm{Na}^{+}$ion concentration inside the anolyte is even higher than that of the $\mathrm{NH}_{4}{ }^{+}$ions. As shown in Figure 6.7, for higher initial concentration of $\mathrm{Na}^{+}$ ions, the backward transport of $\mathrm{Na}^{+}$ions from the catholyte into the anolyte and associated positive 
effect on $\mathrm{NH}_{4}{ }^{+}$ions removal from the anolyte during the early stage of operation decreases moderately. The competition of $\mathrm{Na}^{+}$ions for transport across CEM sets in earlier than that under lower $\mathrm{Na}^{+}$ion concentration in the anolyte. For lower initial concentration of $\mathrm{Na}^{+}$ions, the opposite occurs.

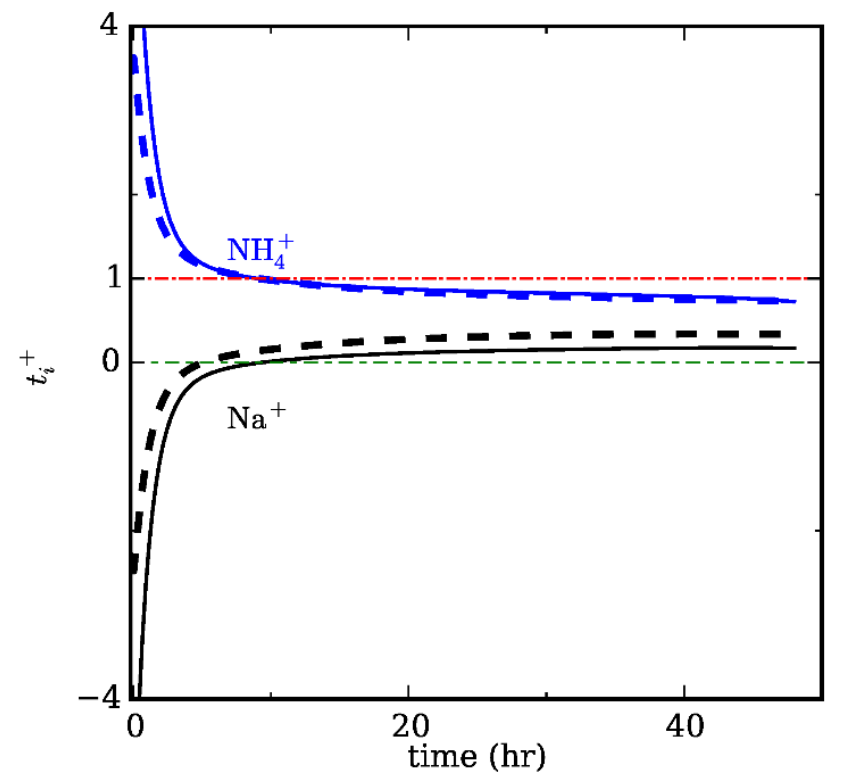

Figure 6.7 Transport number of $\mathrm{Na}^{+}$and $\mathrm{NH}_{4}{ }^{+}$ions at the CEM-anolyte interface under different initial $\mathrm{Na}^{+}$ion concentration in the anolyte. The solid (dashed) lines are for case in which the initial concentration of $\mathrm{Na}^{+}$ions in the anolyte is equal to (three times of) that of the $\mathrm{NH}_{4}{ }^{+}$ions.

To further assess the ion competition effect, we examined the $\mathrm{NH}_{4}{ }^{+}$concentration in anolyte during MEC operation with different initial $\mathrm{Na}^{+}$concentration in the anolyte while keeping all other operating conditions the same. Figure 6.8 shows that, as the initial $\mathrm{Na}^{+}$concentration in the anolyte increases (decreases), less (more) $\mathrm{NH}_{4}{ }^{+}$ions are transported out of the anolyte due to stronger ion competition. Most of difference occurs during the early stage of operation, and the difference in the later stage is small. Overall, the transport of $\mathrm{NH}_{4}{ }^{+}$ions out of the anolyte is affected only 
moderately by the initial $\mathrm{Na}^{+}$ion concentration in the anolyte, suggesting that the ion competition effect is moderate in the system studied here.

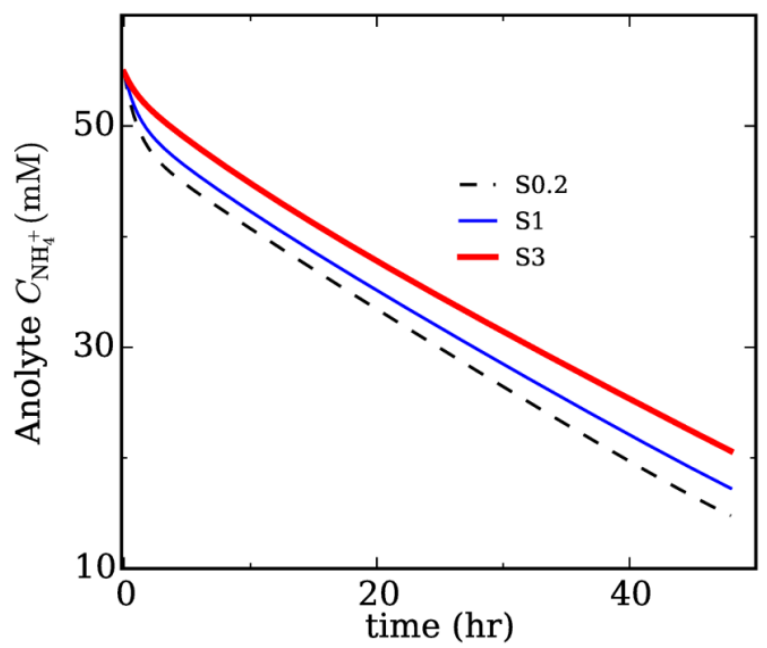

Figure 6.8 Evolution of the $\mathrm{NH}_{4}{ }^{+}$ion concentration in anolyte during operations with different initial $\mathrm{Na}^{+}$ion concentrations in the anolyte. S0.2, S1, and $\mathrm{S3}$ corresponds to the case in which the initial $\mathrm{Na}^{+}$ion concentration in the anolyte is $0.2,1.0$ and 3.0 (for demonstration purpose) times of the initial $\mathrm{NH}_{4}{ }^{+}$ion concentration, respectively. The operating parameters (e.g., current density and aeration rate) are otherwise identical in all cases.

\subsection{Discussion}

Using integrated experimental and simulation studies, we examined the $\mathrm{NH}_{4}{ }^{+} / \mathrm{NH}_{3}$ transport in MECs and their coupling with the current generation, the acid-base reactions, and the transport of inert cations in the system. During MEC operation, a cascade of chemical groups regulates the $\mathrm{pH}$ in system to lead to an acidic (basic) environment in the anolyte (catholyte). The $\mathrm{NH}_{4}{ }^{+} / \mathrm{NH}_{3}$ couple is found to play a dual role in the operation: it serves as an effective proton shuttle for the charge transport across the CEM and also as buffer agent in the anolyte and catholyte. Inert cations, even in abundance, compete rather moderately with $\mathrm{NH}_{4}{ }^{+}$ion for transport across the CEM. The strength 
of the diffusion of $\mathrm{NH}_{3}$ from catholyte to anolyte is governed by the MEC's operating conditions and can be estimated using a dimensionless number.

The findings of this study will potentially impact the ammonia recovery using BES in several aspects. First, a clear understanding of ion transport and interaction will help interpret $\mathrm{NH}_{4}^{+}$ion recovery efficiency that may be affected by the composition of wastewater. Second, delineating the role of different chemical groups in $\mathrm{pH}$ regulation will help guide the BES operation for optimal $\mathrm{pH}$. Third, the dimensionless number of diffusion of $\mathrm{NH}_{3}$ will help select suitable membranes. In this work, ammonium removal and recovery was examined under a high-current condition, because high current generation was expected to benefit the transport of ammonium ions. Reducing current generation via lowering applied voltage and/or increasing external resistance would decrease ammonium removal/recovery.

There are still challenges to address in the future to improve the agreement between the model and the experiment data. For example, there is a slight volume loss of the catholyte due to the aeration, and this effect was not considered in our model but it is likely to affect the concentration prediction in the catholyte to some extent. The bacteria activity will need to be considered in the future work. The bacteria activity in the anode could affect the balance of most of the species within the system, especially $\mathrm{NH}_{4}{ }^{+}$and $\mathrm{Na}^{+}$, both of which are important to ion competition.

Future studies will focus on systematic investigation of the role of current in ion transport and ammonia recovery, the interaction between electric potential and ammonium recovery, improving the numerical model by integrating current generation models into the existing model, and extension of the unsteady model to other BES systems. 


\title{
Chapter 7 Integrated Experimental and Modeling Evaluation of Energy Consumption for Ammonia Recovery in Bioelectrochemical Systems
}

(This section has been published as Qin, M., Liu, Y. (Co-first author), Luo, S., Qiao, R., * \& He, Z.* (2017). Integrated Experimental and Modeling Evaluation of Energy Consumption for Ammonia Recovery in Bioelectrochemical Systems. Chemical Engineering Journal, 327, 924-931.)

\begin{abstract}
Bioelectrochemical systems (BES) can recover ammonia from wastewater driven by electricity generation. However, energy consumption of such an approach has not been well evaluated. In this study, the effects of several key operating factors including catholyte aeration rate, external voltage, and external resistance on both ammonia recovery and energy consumption were systematically investigated. A mathematical model developed for ammonia removal/recovery in BES was applied to help interpret the experimental results. It was found that a high aeration rate in the catholyte could facilitate ammonia recovery. An aeration rate of $100 \mathrm{~mL} \mathrm{~min}{ }^{-1}$ resulted in the lowest energy consumption of $4.9 \mathrm{kWh} \mathrm{kg}^{-1} \mathrm{~N}$ recovery among the tested aeration rates. A low external resistance facilitated the ammonia recovery via higher current generation, while a moderate external voltage (e.g., $0.5 \mathrm{~V}$ ) helped to achieve low energy consumption. The highest ammonia recovery rate of $7.1 \mathrm{~g} \mathrm{~N} \mathrm{~m}^{-2} \mathrm{~d}^{-1}$ was obtained with energy consumption of $5.7 \mathrm{kWh} \mathrm{kg}^{-1}$ $\mathrm{N}$ recovery. Therefore, there is a trade-off between energy consumption and ammonia recovery.
\end{abstract}




\subsection{Introduction}

Nitrogen is a key inorganic contaminant, and excessive discharge of nitrogen, for example via incomplete wastewater treatment, can amplify the nitrogen transfer to aquatic ecosystems and result in eutrophication that deteriorates ecosystem and decreases the supply of drinking water source (Howarth et al. 1996). The annual cost due to eutrophication in the United States is about \$2200 million (Dodds et al. 2008). Thus, removal of nitrogen from wastewater is necessary to limit nitrogen discharge. In wastewater, the primary form of nitrogen is ammonium (Matassa et al. 2015b), which can be removed by either biological or physicochemical methods (Ahn 2006b, Sprynskyy et al. 2005). In biological ammonium removal processes such as nitrification, denitrification and anaerobic ammonium oxidation (anammox), ammonium is converted to nitrogen gas microbiologically. The physicochemical ammonium removal processes like ion exchange, air stripping and high gravity separate ammonia from liquid at the expense of chemical or energy consumption (Gupta et al. 2015, Jiao et al. 2016, Wang et al. 2017).

Sustainable wastewater management aims to recover valuable resources from wastewater, such as energy, nutrients and water. The concentration of ammonium in some wastewaters such as digester centrate and landfill leachate can be over $500 \mathrm{mg} \mathrm{L}^{-1}$ (Renou et al. 2008). Thus, ammonia recovery, instead of removal, will be of strong interest. Ammonia nitrogen is a key fertilizer component for agricultural applications, and more than $90 \%$ of the world ammonia production is currently based on the Haber-Bosch synthesis process, which consumes 1-2\% of world energy (Capodaglio et al. 2015). The demand for fertilizer is increasing at 3-4\% per year along with the steadily growing global demand for food to feed additional 2.3 billion people by 2050 (Bicer et al. 2016, Cordell et al. 2009). About $30 \%$ of ammonia in fertilizers ends up in wastewater as ammonium ions 
(Ledezma et al. 2015). Therefore, recovering ammonia from wastewater may provide a promising approach for supplying ammonia to sustain fertilizer and food production, and avoid energyintensive synthesizing processes (Verstraete et al. 2009).

An emerging technology for ammonia recovery from wastewater is bioelectrochemical system (BES) (Arredondo et al. 2015, Grant et al. 2012). In a BES, organic compounds in wastewater are oxidized by the exoelectrogens growing on an anode electrode and the generated electrons spontaneously flow from the anode electrode to a cathode electrode (Kelly and He 2014). During electricity generation, ammonium ions are driven to transport from the anode to the cathode to maintain charge neutrality (Wang and Ren 2013). The high $\mathrm{pH}$ of the catholyte due to reduction reactions converts ammonium ions to ammonia, which can be driven off the catholyte with mechanical mixing or aeration for recovery. Various BES including microbial fuel cells (MFCs) and microbial electrolysis cells (MECs) have been studied to achieve ammonia recovery (CordRuwisch et al. 2011, Wu et al. 2017). Because higher current generation would greatly enhance ammonium transport towards recovery (Kelly and He 2014), MECs with external power exhibit a better performance for ammonia recovery than MFCs (Haddadi et al. 2013). Ammonium nitrogen has been successfully recovered as ammonia from ammonium-rich wastewater, such as synthetic wastewater, urine, landfill leachate, and swine wastewater (Kim et al. 2008, Kuntke et al. 2011, Kuntke et al. 2012, Qin and He 2014, Qin et al. 2016b, Zhang et al. 2014a). Mathematical models have also been developed to improve our understanding of ion transport mechanism during ammonia recovery and the BES operation (Dykstra et al. 2014c, Liu et al. 2016, Recio-Garrido et al. 2016). 
Despite the great progress in ammonia recovery by using BES, energy consumption of such a process has not been fully investigated before. This information is critically important to evaluate whether ammonia recovery by using BES will be energy efficient (and cost effective). An energyefficient treatment and recovery system will benefit our environment with contaminant removal, reduced demand for energy (and thus lower carbon footprint), and reduced demand for raw materials through resource recovery. The major energy consumers in a BES include power supply, recirculation pumps, and catholyte aeration (Dong et al. 2015). Energy consumption by power supply can be affected by both voltage and external resistance via current generation, which then influences ammonium ion transport. Electrolyte recirculation helps with substrate distribution that will affect current generation and electrolyte resistance. Catholyte aeration is to provide electron acceptors for cathode reactions, and to strip ammonia off the liquid for subsequent recovery. To improve our understanding of energy consumption of ammonia recovery by using BES, in this study, we have systematically investigated the effects of catholyte aeration rate, external voltage, and external resistance on both ammonia recovery and energy consumption. A mathematical model was applied to verify and further explain the effects of the operation parameters on the BES performance and energy consumption. The energy consumption by the BES was normalized to per $\mathrm{kg} \mathrm{N}$ removal or recovery.

\subsection{Materials and Methods}

\subsubsection{BES setup and operation}

A bench-scale cubic shape BES was used in this study. Both the anode and the cathode chambers had the same dimension of $9 \mathrm{~cm} \times 4.7 \mathrm{~cm} \times 1 \mathrm{~cm}$. A piece of cation exchange membrane (CEM, CMI-7000, Membrane International Inc., Glen Rock, NJ, USA) with a sectional area of $42.3 \mathrm{~cm}^{2}$ 
was used to separate the anode and cathode chambers. The anode electrode was a carbon brush (Gordon Brush Mfg. Co., Inc., CA, USA), which was pretreated by being soaked in pure acetone overnight and heat-treated in a muffle furnace (Model 550 Isotemp Series, Fisher Scientific, Pittsburgh, PA, USA) at $450{ }^{\circ} \mathrm{C}$ for $30 \mathrm{~min}$. The cathode electrode was a piece of $32-\mathrm{cm}^{2}$ carbon cloth (Zoltek Companies, Inc., MO, USA) that was coated with $5 \mathrm{mg} \mathrm{cm}^{-2}$ of Pt/C (10\% wt. Pt on Carbon Vulcan, Fuel Cell Earth LLC, USA). The BES was operated at room temperature of $\sim 21{ }^{\circ} \mathrm{C}$.

The anode was inoculated with the anaerobic sludge from a local wastewater treatment plant (Peppers Ferry, Radford, VA, USA). To mimic the digestion effluent of livestock waste (Park et al. 2010), the anode influent solution was prepared containing (per liter of deionized water - DI water): sodium acetate, $1.5 \mathrm{~g} ; \mathrm{NH}_{4} \mathrm{Cl}, 3.0 \mathrm{~g} ; \mathrm{NaHCO}_{3}, 2.0 \mathrm{~g} ; \mathrm{NaCl}, 0.15 \mathrm{~g} ; \mathrm{MgSO}_{4}, 0.005 \mathrm{~g} ; \mathrm{CaCl}_{2}$, $0.006 \mathrm{~g}$; and trace elements solution, $1 \mathrm{~mL}$ (Angenent and Sung 2001). The anolyte volume was $100 \mathrm{~mL}$, while the cathode chamber was initially filled with $180 \mathrm{~mL}$ of DI water. An external voltage of $0.8 \mathrm{~V}$ was applied to the circuit by a power supply (CSI3644A, Circuit Specialists, Inc., Mesa, AZ, USA) according to a previous study (Angenent and Sung 2001). The BES was operated in a batch mode at room temperature $\left(\sim 20^{\circ} \mathrm{C}\right)$. When current generation dropped to lower than 0.2 A $\mathrm{m}^{2}$, one batch cycle ended and the anolyte was partially replaced (75\% of the anode volume) while the catholyte was completely replaced with $180 \mathrm{~mL}$ of DI water. Both the anolyte and catholyte were recirculated at a flow rate of $20 \mathrm{~mL} \mathrm{~min}^{-1}$, respectively. Samples $(1 \mathrm{~mL})$ were collected every 4 hours from both chambers for measurement. The stripped $\mathrm{NH}_{3}$ gas from the cathode was absorbed by $1 \mathrm{M} \mathrm{H}_{2} \mathrm{SO}_{4}$. To study the effect of aeration rate, the catholyte aeration rates were adjusted to $300,100,50$ and $30 \mathrm{~mL} \mathrm{~min}^{-1}(1.7,0.6,0.3$ and $0.2 \mathrm{vvm}$; vvm: gas volume 
(L) per liquid volume (L) per minute). In the study of current generation, two experiments were performed: in the test of external voltage effect, three external voltages $0 \mathrm{~V}, 0.5 \mathrm{~V}$, and $0.8 \mathrm{~V}$, were examined across external resistance of $1 \Omega$ and with the catholyte aeration rate of $100 \mathrm{~mL} \mathrm{~min}^{-1}$ (0.6 vvm); in the experiment of external resistance, the external resistance was manipulated at three levels, $1 \Omega, 10 \Omega$, and $100 \Omega$, under the applied voltage of $0.8 \mathrm{~V}$ and with the catholyte aeration rate of $100 \mathrm{~mL} \mathrm{~min}^{-1}$.

\subsubsection{Measurement and analysis}

The voltage across the resistor was recorded every 2 min by a digital multimeter (2700, Keithley Instruments Inc., Cleveland, OH, USA). The $\mathrm{pH}$ was measured by two $\mathrm{pH}$ meters (Oakton Instruments, Vernon Hills, IL, USA and Accumet AB250, Fisher Scientific, Pittsburgh, PA, USA), installed in the anode and the cathode chambers, respectively. The concentrations of chemical oxygen demand (COD) and ammonium nitrogen $\left(\mathrm{NH}_{4}{ }^{+}-\mathrm{N}\right)$ were measured using a DR/890 colorimeter (HACH Co., Ltd., USA) according to the manufacturer's instruction. Energy consumption by the BES included the external power source, the aeration, and the recirculation of the anolyte and catholyte. The energy input by the external power supply was calculated as (Logan 2008):

$$
P_{\text {power }}=\frac{I U}{1000}
$$

where $P_{\text {power }}$ is external power requirement by the power supply $(\mathrm{kW}) ; I$ is the current $(\mathrm{A})$; and $U$ is the external voltage $(\mathrm{V})$.

The theoretical power requirement for the recirculation was estimated as (Yang et al. 2016c): 


$$
P_{\text {recirculation }}=\frac{Q_{r} \gamma E}{1000}
$$

where $P_{\text {recirculation }}$ is the pumping system power requirement $(\mathrm{kW}) ; Q_{s}$ is the recirculation flow rate $\left(\mathrm{m}^{3} \mathrm{~s}^{-1}\right) ; \gamma$ is specific weight of water $\left(9800 \mathrm{~N} \mathrm{~m}^{-3}\right)$; and $E$ is the hydraulic pressure head $(\mathrm{m})$.

The energy consumption by the aeration was estimated as (Verrecht et al. 2008):

$$
P_{\text {aeration }}=\frac{P_{1} \lambda T}{3.6 \times 2.73 \times 10^{8} \times \zeta \times(\lambda-1) \times \rho_{0}} \times\left(\left(\frac{P_{2}}{P_{1}}\right)^{1-1 / \lambda}-1\right) \times Q_{g}
$$

where $P_{\text {aeration }}$ is the aeration power requirement $(\mathrm{kW}) ; P_{1}$ is standard atmospheric pressure $(101,325 \mathrm{~Pa}) ; P_{2}$ is blower inlet pressure $(\mathrm{Pa}) ; T$ is air temperature $(294 \mathrm{~K}) ; \zeta$ is blower efficiency (0.8); $\lambda$ is aerator constant (1.4); $\rho_{0}$ is air density at the standard conditions $\left(1.29 \mathrm{~kg} \mathrm{~m}^{-3}\right)$; and $Q_{g}$ is the aeration flow rate $(\mathrm{mol} / \mathrm{s})$.

The total energy consumption was estimated as:

$$
\boldsymbol{E}=\frac{\left(\boldsymbol{P}_{\text {power }}+\boldsymbol{P}_{\text {aeration }}+\sum \boldsymbol{P}_{\text {recirculation }}-\boldsymbol{I}^{2} \boldsymbol{R}\right)}{\boldsymbol{Q}_{t}}
$$

where the $E$ represents the energy consumption $\left(\mathrm{kWh} \mathrm{m}^{-3}\right)$; and $Q_{t}$ is the flow rate for the treated wastewater $\left(\mathrm{m}^{3} \mathrm{~h}^{-1}\right)$.

The energy data were expressed in $\mathrm{kWh} \mathrm{kg}^{-1} \mathrm{~N}$ removal and $\mathrm{kWh} \mathrm{kg}^{-1} \mathrm{~N}$ recovery (Desloover et al. 2012). The energy consumption per $\mathrm{kg} \mathrm{N}$ removal $\left(E_{C, \text { removal }}\right)$ was calculated as:

$$
E_{C, \text { removal }}=\frac{E}{r_{N, \text { removal }}}
$$

where the $r_{N}$, removal is the $\mathrm{N}$ removal rate $\left(\mathrm{kg} \mathrm{m}^{-3}\right)$ based on the removal from the anode.

The energy consumption per $\mathrm{kg} \mathrm{N}$ recovery was calculated as: 


$$
\boldsymbol{E}_{C, \text { recovery }}=\frac{E}{r_{N, \text { recovery }}}
$$

where the $r_{N}$, recovery is the $\mathrm{N}$ recovery rate $\left(\mathrm{kg} \mathrm{m}^{-3}\right)$ based on the recovery from the whole BES.

\subsubsection{Mathematical model of BES-based ammonia recovery}

To facilitate the understanding of the effects of operating factors on the BES operation and ammonia recovery, numerical simulations were also performed using models that were developed and validated previously (Liu et al. 2016). The BES was modeled as three components, an anode chamber, a cathode chamber, and a CEM. In each component, transport and reaction of all major species in the experimental BES, i.e., $\mathrm{Na}^{+}, \mathrm{Cl}^{-}, \mathrm{HAc}, \mathrm{Ac}^{-}, \mathrm{NH}_{4}^{+}, \mathrm{NH}_{3}, \mathrm{H}_{2} \mathrm{CO}_{3}, \mathrm{HCO}_{3}-, \mathrm{CO}_{3}{ }^{2-}, \mathrm{H}^{+}$ and $\mathrm{OH}^{-}$, are considered. In the anolyte/catholyte chamber, evolution of the concentration of each species was modeled by considering mass conservation, its transport toward/from the CEM, the acid-base reactions involved, and the electrochemical reactions associated with the Faraday current. In the CEM, in addition to the acid-base reactions, the transport of each species by diffusion and migration was modeled using the Poisson-Nernst-Planck equations. Donnan equilibrium was enforced at the CEM-anolyte/catholyte interface. To model the recovery of ammonia from the catholyte due to aeration, the Henry's law was used to describe the equilibrium between the $\mathrm{NH}_{3}$ in the catholyte and the aeration gas. Finally, electro-neutrality and charge conservation were enforced throughout the system.

The concentration evolution of the following species is considered: $\mathrm{Na}^{+}, \mathrm{Cl}^{-}, \mathrm{HAc}, \mathrm{Ac}^{-}, \mathrm{NH}_{4}^{+}, \mathrm{NH}_{3}$, $\mathrm{H}_{2} \mathrm{CO}_{3}, \mathrm{HCO}_{3}{ }^{-}, \mathrm{CO}_{3}{ }^{2-}, \mathrm{H}^{+}$and $\mathrm{OH}^{-}$. The system is divided into three parts: the anode chamber, the cathode chamber, and the membrane (CEM in this case). The average concentration of species $i$ inside each chamber follows 


$$
V_{j} \frac{\partial C_{i}^{j}}{\partial t}=A \cdot J_{i, j}^{\prime}-\dot{R}_{i, j}^{a}+\dot{S}_{i, j}^{c} V_{j}
$$

where $V_{j}$ is the volume of chamber $j$ ( $j=1$ : anode chamber; $j=2$ cathode chamber), $C_{i}^{j}$ is the concentration of species $i$ in chamber $j, A$ is the area of the CEM, and $J_{i, j}^{\prime}$ is the flux of species $i$ into chamber $j . \dot{R}_{i, j}^{a}$ is the removal of species $i$ from chamber $j$ due to aeration, which is determined by assuming fast equilibrium between species dissolved in the catholyte and existing in the aeration gas (Henry's Law). $\dot{S}_{i, j}^{c}$ is the generation/removal rate (per volume) of the species $i$ due to chemical/biological reactions, respectively. For example, for the $\mathrm{NH}_{3}, \mathrm{NH}_{4}{ }^{+}$and $\mathrm{H}^{+}$concentration, relevant generation/removal needs to be included because of the acid-base equilibrium $\mathrm{NH}_{4}{ }^{+} \leftrightarrow \mathrm{NH}_{3}+\mathrm{H}^{+}$. A list of all acid-base equilibrium is provided in Table 7.1.

The CEM is resolved spatially in its thickness direction. Each species $i$ at $x=0$ (anode/CEM interface) and $L$ (CEM/cathode interface) is always in equilibrium with that in the anode and cathode chambers, respectively. The distribution of each species $i$ across the CEM, $C_{i}^{m}$, is governed by

$$
\frac{\partial C_{i}^{m}}{\partial t}=\dot{S}_{i, m}^{c}+\nabla \cdot J_{i, m}
$$

where $\dot{S}_{i, m}^{c}$ is the generation/consumption of species $i$ by acid-base equilibrium. $J_{i, m}$ is the flux of species $i$ in the membrane given by the Nernst-Planck equation:

$$
J_{i, m}=-D_{i}^{m}\left(\frac{\partial C_{i}^{m}}{\partial x}+z_{i} C_{i}^{m} \frac{e}{k_{B} T} \frac{\partial \phi}{\partial x}\right)
$$

where $D_{i}^{m}$ and $z_{i}$ are the diffusion coefficient in the membrane and valence of species $i$, respectively; $e$ and $k_{B} T$ are the electron charge and thermal energy; $\phi$ is the electrical potential. Because the concentration of various species inside the anode/cathode chambers evolves slowly at 
a time scale of tens of hours but the relaxation of species concentration within the CEM is fast due to CEM's small thickness, the time dependence term in Eq. (Equation 6.2) is unimportant and thus neglected.

The concentration distribution of any species $i$ across the anolyte/catholyte-CEM interfaces is treated as follows. For electrically neutral species, their concentrations are continuous across these interfaces. For a charged species $i$, the Donnan equilibrium condition is used, i.e.,

$$
C_{i}^{\mathrm{m}}=\exp \left(-z_{i} e \Delta \phi / k_{B} T\right) C_{i}^{j}
$$

(Equation 7.10)

where $\Delta \phi$ is the electrical potential drop across the CEM-solution interface (usually termed the Donnan potential). Note that the Donnan potential attracts cations into (repels anions from) the CEM so that the negative fixed charge inside the membrane is balanced by the free ions.

Finally, the electro-neutrality condition and the charge conservation law are enforced:

$$
\begin{gathered}
\omega X+\sum_{i} Z_{i} C_{i}=0 \\
I=F \Sigma_{i} Z_{i} J_{i}
\end{gathered}
$$

where $\omega$ is the sign of the fixed space charge in any domain $(\omega=0$ in the anode/cathode chamber and zero charge membranes; $\omega=-1$ and +1 in cation and anion exchange membranes, respectively). $X$ is the fixed charge density of each domain. $F$ and $I$ are the Faraday constant and the current density through the CEM, respectively.

Table 7.1 Acid-base reactions and gas-solution equilibrium considered in our model.

\begin{tabular}{|l|l|l|c|l|l|}
\hline & Constant & Reaction & Equilibrium equation & Value & Notes \\
\hline & $\mathrm{K}_{\mathrm{a}, \mathrm{Ac}}$ & $\mathrm{HAc} \leftrightarrow \mathrm{Ac}^{-}+\mathrm{H}^{+}$ & $K_{\mathrm{a}, \mathrm{Ac}}=\frac{C_{\mathrm{Ac}^{-}} \cdot C_{\mathrm{H}^{+}}}{C_{\mathrm{HAc}}}$ & 0.0174 & $\begin{array}{l}\text { Taken } \\
\text { from }\end{array}$ \\
\cline { 2 - 6 } & $\mathrm{K}_{\mathrm{a}, \mathrm{CA} 1}$ & $\begin{array}{l}\mathrm{H}_{2} \mathrm{CO}_{3} \leftrightarrow \mathrm{H}^{+}+\mathrm{HCO} \\
3^{-}\end{array}$ & $K_{\mathrm{a}, \mathrm{CA} 1}=\frac{C_{\mathrm{H}^{+}} \cdot C_{\mathrm{HCO}_{3}^{-}}}{C_{\mathrm{H}_{2} \mathrm{CO}_{3}}}$ & $4.4668 \times 10^{-4}$ & $\begin{array}{l}\text { Ref. } \\
\text { (Dykstr } \\
\text { Acid-base } \\
\text { reaction }\end{array}$ \\
\cline { 2 - 6 } & $\mathrm{K}_{\mathrm{a}, \mathrm{CA} 2}$ & $\mathrm{HCO}_{3} \leftrightarrow \leftrightarrow \mathrm{H}^{+}+\mathrm{CO}_{3}{ }^{2-}$ & $K_{\mathrm{a}, \mathrm{CA} 2}=\frac{C_{\mathrm{H}^{+}} \cdot C_{\mathrm{CO}_{3}^{2-}}}{C_{\mathrm{HCO}_{3}^{-}}}$ & $4.6774 \times 10^{-8}$ & $\begin{array}{l}\text { al. } \\
\text { 2014a) }\end{array}$ \\
\hline
\end{tabular}




\begin{tabular}{|c|c|c|c|c|}
\hline & $\mathrm{K}_{\mathrm{a}, \mathrm{NH}}$ & $\mathrm{NH}_{4}{ }^{+} \leftrightarrow \mathrm{NH}_{3}+\mathrm{H}^{+}$ & $K_{\mathrm{a}, \mathrm{NH}}=\frac{C_{\mathrm{H}^{+}} \cdot C_{\mathrm{NH}_{3}}}{C_{\mathrm{NH}_{4}^{+}}}$ & $5.6234 \times 10^{-7}$ \\
\hline & $\mathrm{K}_{\mathrm{w}}$ & $\mathrm{H}_{2} \mathrm{O} \leftrightarrow \mathrm{H}^{+}+\mathrm{OH}^{-}$ & $K_{\mathrm{w}}=C_{\mathrm{H}^{+}} \cdot C_{\mathrm{OH}^{-}}$ & $1 \times 10^{-8}$ \\
\hline \multirow{2}{*}{$\begin{array}{l}\text { Gas- } \\
\text { solution } \\
\text { equilibriu } \\
\mathrm{m}\end{array}$} & $\mathrm{K}_{\mathrm{H}, \mathrm{CO}_{2}}$ & & $K_{\mathrm{H}, \mathrm{CO}_{2}}=\frac{C_{\mathrm{CO}_{2}, \mathrm{aq}}}{p_{\mathrm{CO}_{2}}}$ & 33.46 \\
\hline & $\mathrm{K}_{\mathrm{H}, \mathrm{NH}_{3}}$ & & $K_{\mathrm{H}, \mathrm{NH}_{3}}=\frac{C_{\mathrm{NH}_{3}, \mathrm{aq}}}{p_{\mathrm{NH}_{3}}}$ & 56250 \\
\hline
\end{tabular}

*The values correspond to a concentration unit of $\mathrm{mol} / \mathrm{m}^{3}$ and a pressure unit of atm;

\subsection{Results and Discussion}

\subsubsection{Effects of aeration rate on ammonia recovery}

The influence of catholyte aeration rates on the BES performance and ammonia recovery was investigated by varying the aeration rate from 300 to $30 \mathrm{~mL} \mathrm{~min}^{-1}$. The BES exhibited a relatively stable current generation at different aeration rates (Figure 7.1A) with the total Coulomb production of $\sim 350 \mathrm{C}$ (Figure 7.2 ), which was not significantly different among all the tested aeration rates $(p>0.05)$. Likewise, the COD removal was also insignificantly different $(p>0.05)$, and the COD concentration decreased from $1302 \pm 49 \mathrm{mg} \mathrm{L}^{-1}$ to $258 \pm 23 \mathrm{mg} \mathrm{L}^{-1}$ (mean value \pm standard deviation), representing COD removal efficiency of $80.4 \pm 2.4 \%$. In a batch cycle, the ammonium concentration in the anolyte decreased from $55 \pm 2 \mathrm{mM}$ to $22 \pm 6 \mathrm{mM}, 22 \pm 5 \mathrm{mM}, 24$ $\pm 3 \mathrm{mM}, 36 \pm 7 \mathrm{mM}$, with aeration rate of $300,100,50$ and $30 \mathrm{~mL} \mathrm{~min}^{-1}$, respectively (Figure 7.1B). The ammonium removal was not significantly different $(p>0.05)$ among the aeration rates of 300,100 and $50 \mathrm{~mL} \mathrm{~min}{ }^{-1}$, but the lowest aeration rate of $30 \mathrm{~mL} \mathrm{~min}^{-1}$ resulted in dramatic decrease in the removal. A higher aeration rate could promote ammonia recovery: the recovery rate of ammonia gas reached $7.1 \pm 0.2 \mathrm{~g} \mathrm{~N} \mathrm{~m}^{-2} \mathrm{~d}^{-1}(\mathrm{~kg} \mathrm{~N}$ recovery per membrane area per day) with the aeration rate of $300 \mathrm{~mL} \mathrm{~min}^{-1}$, much higher than $1.2 \pm 0.1 \mathrm{~g} \mathrm{~N} \mathrm{~m}^{-2} \mathrm{~d}^{-1}$ with $30 \mathrm{~mL} \mathrm{~min}^{-1}$ (Figure 7.1C). Those results suggested that the aeration rate in the catholyte had limited effects on current 
generation and COD removal, but could strongly affect the ammonium removal and ammonia recovery. As shown in Figure 7.1C, there is no significant difference for ammonia recovery rate at the aeration rates of 100 and $300 \mathrm{ml} \mathrm{min}^{-1}$, likely because that the high aeration rate provided a sufficient stripping force to remove ammonia gas from the catholyte and was no longer a limiting factor for ammonium recovery in the BES.
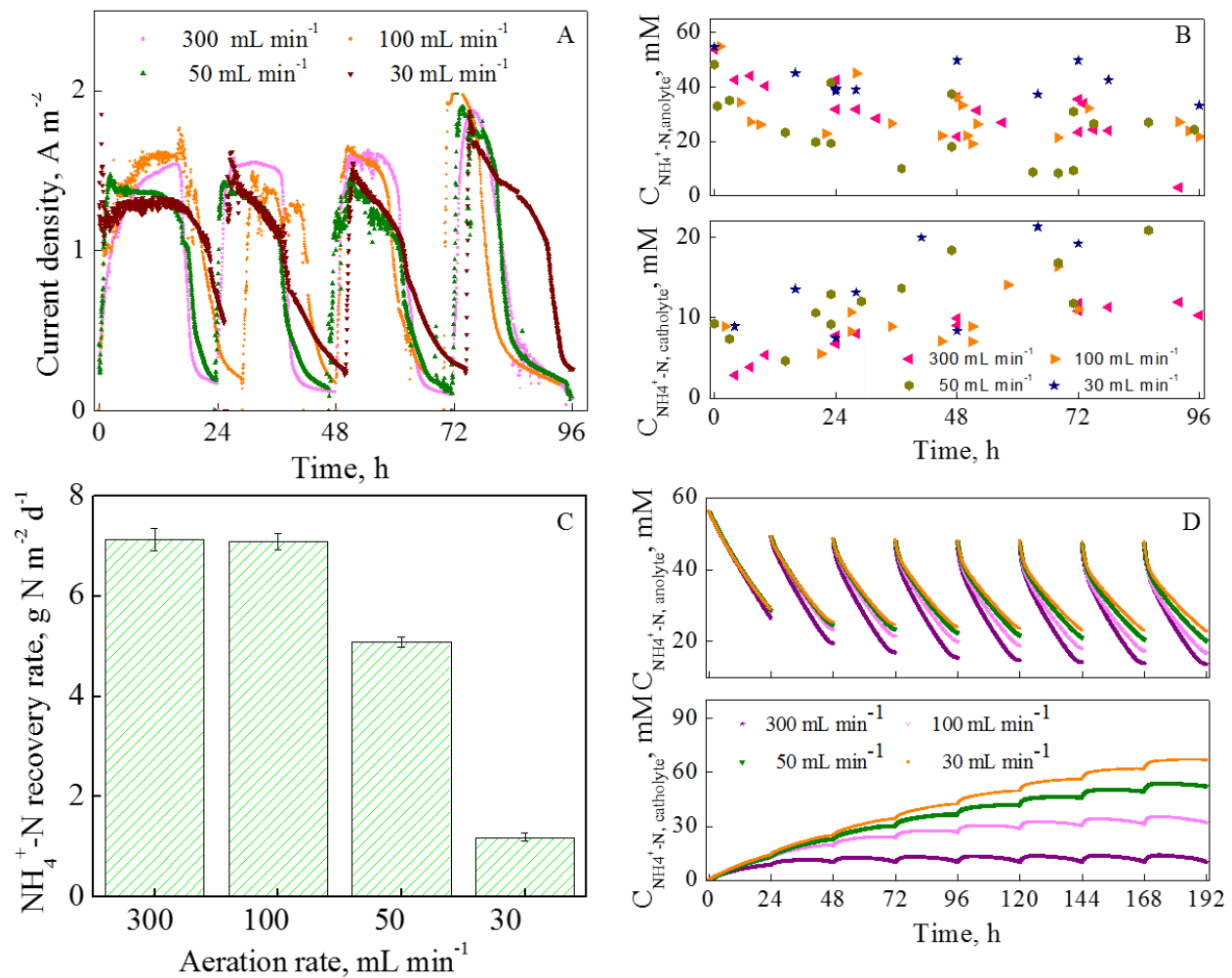

Figure 7.1 Ammonia recovery by the BES affected by different catholyte aeration rates: (A) current generation; $(B) \mathrm{NH}_{4}{ }^{+}-\mathrm{N}$ concentration in the anolyte and the catholyte; (C) the $\mathrm{NH}_{3}$ recovery rate; and (D) simulated anolyte and catholyte $\mathrm{NH}_{4}{ }^{+}-\mathrm{N}$ concentration. The external resistance was $1 \Omega$ and the external voltage was $0.8 \mathrm{~V}$.

To further understand the mechanism of the effects, mathematical simulation was performed under the similar conditions, i.e., fixed current density of $1 \mathrm{~A} \mathrm{~m}^{-2}$ for 24 hours with different aeration rates. A similar trend to that of experimental results was observed: a higher aeration rate led to a higher ammonium removal from the wastewater (anolyte) to the catholyte and a lower ammonium concentration in the catholyte (Figure 
7.1D), which resulted in higher ammonia recovery. The ammonium concentration in the anolyte effluent decreased from $25.7 \pm 1.9 \mathrm{mM}$ to $19.6 \pm 4.2 \mathrm{mM}$ when the aeration rate increased from 30 to $300 \mathrm{~mL} \mathrm{~min}^{-}$ ${ }^{1}$, while the ammonium concentration in the catholyte at the end of each operating cycle decreased from $61.5 \pm 4.4 \mathrm{mM}$ to $10.1 \pm 0.1 \mathrm{mM}$. The simulation results also showed that the recovery of ammonia directly depended on both the aeration rate and the related catholyte ammonium concentration (Henry's law) (Dasgupta and Dong 1986). A high aeration rate was effective in driving more ammonia out of the catholyte (Figure 7.3), leading to a lower ammonium concentration in the catholyte, which in turn facilitated the ammonium removal from the anolyte through both migration and diffusion of $\mathrm{NH}_{4}{ }^{+}$ions from the anolyte to the catholyte (Figure 7.1D). As a result, the ammonia recovery from each batch cycle increased from 1.5 $\pm 0.1 \mathrm{mmol}$ to $3.2 \pm 0.1 \mathrm{mmol}$ when the aeration rate increased from 30 to $300 \mathrm{~mL} \mathrm{~min}{ }^{-1}$.

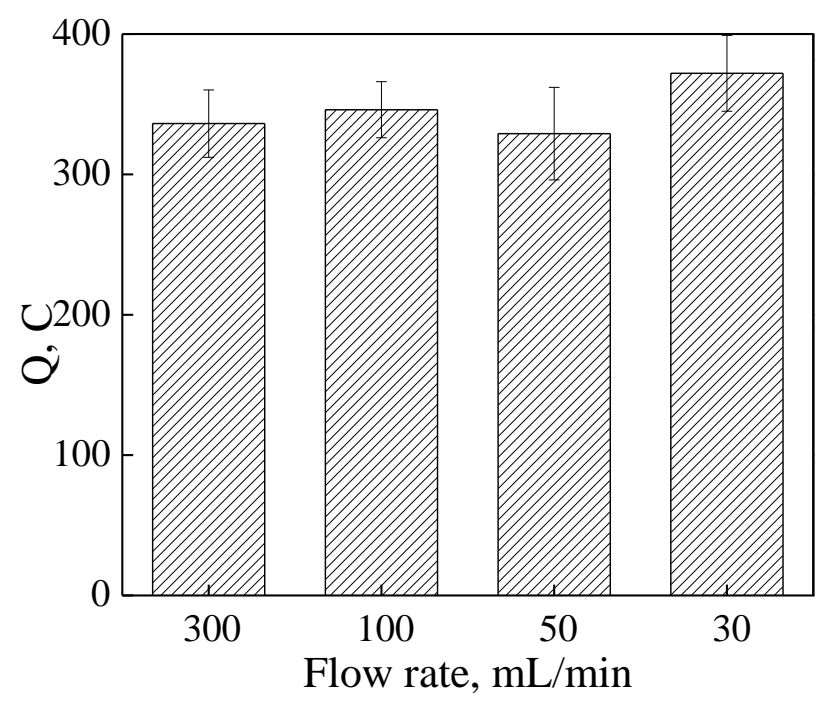

Figure 7.2 The total Coulomb generation per cycle for BES with different aeration rate. 


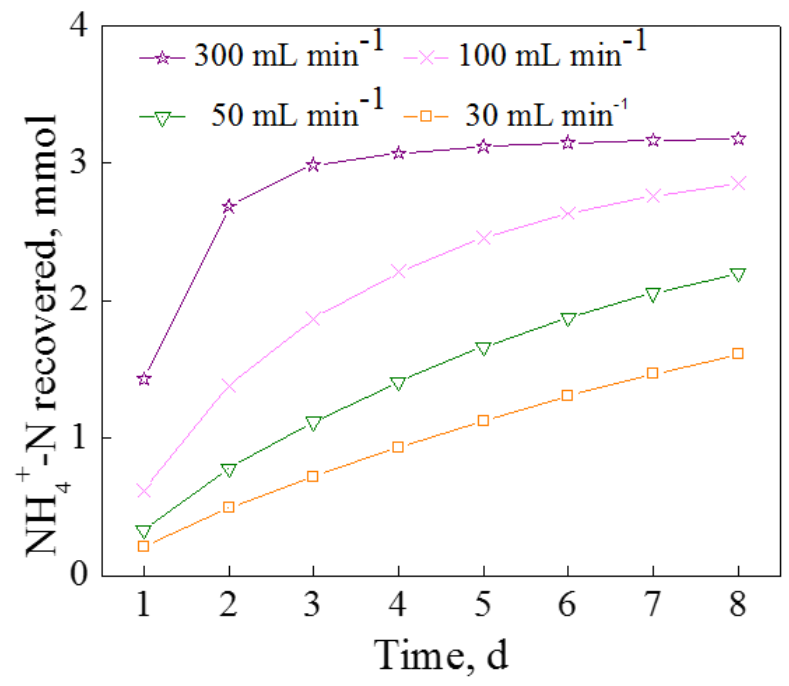

Figure 7.3 The simulated amount of $\mathrm{NH}_{3}$ collected from catholyte by aeration with different catholyte aeration rate

Energy consumption was analyzed for ammonium removal from the anode chamber and ammonia recovery from the whole BES, respectively (Figure 7.4). The removal required $5.1 \pm 0.4,4.3 \pm 0.2$, $4.1 \pm 0.1$, and $6.9 \pm 0.7 \mathrm{kWh} \mathrm{kg}^{-1} \mathrm{~N}$ when the aeration rate was $300,100,50$ and $30 \mathrm{~mL} \mathrm{~min}^{-1}$, respectively, in which over $90 \%$ was consumed by the external power supply and catholyte aeration (Figure 7.4A). For example, with the aeration rate of $300 \mathrm{~mL} \mathrm{~min}^{-1}, 50.0 \%$ of energy consumption for ammonium removal was due to the aeration, $43.5 \%$ was consumed by the external power supply, and $6.5 \%$ was due to the anolyte and catholyte recirculation. As increasing the aeration rate from 30 to $100 \mathrm{~mL} \mathrm{~min}^{-1}$, the energy consumption was decreased due to the improved ammonium removal efficiency. When the aeration rate increased from 100 to $300 \mathrm{~mL} \mathrm{~min}^{-1}$, the ammonium removal was not significantly different, and therefore the increased energy consumption due to more aeration resulted in high total energy consumption. Ammonia recovery required $5.7 \pm 0.5,4.9 \pm 0.3,6.1 \pm 0.4$, and $26.2 \pm 1.1 \mathrm{kWh} \mathrm{kg}^{-1} \mathrm{~N}$ when the aeration rate was 300 , 
100,50 and $30 \mathrm{~mL} \mathrm{~min}^{-1}$, respectively, which was higher than the energy consumption for removal at the same aeration rate, especially when the aeration rate was $30 \mathrm{~mL} \mathrm{~min}^{-1}$ (Figure 7.4B). With the same aeration rate, the energy consumption for recovery was higher than that for removal, because some of the removed ammonium was not successfully recovered and thus still stayed in the catholyte. The ammonia recovery rate was $7.1 \pm 0.2,7.1 \pm 0.1,5.1 \pm 0.1$, and $1.2 \pm 0.1 \mathrm{~kg} \mathrm{~N}$ $\mathrm{m}^{-2} \mathrm{~d}^{-1}$, when the aeration rate was $300,100,50$ and $30 \mathrm{~mL} \mathrm{~min}^{-1}$, respectively. The low ammonia recovery rate at $30 \mathrm{~mL} \mathrm{~min}{ }^{-1}$ aeration rate led to the significantly high energy consumption of 26 . $2 \pm 1.1 \mathrm{kWh} \mathrm{kg}^{-1} \mathrm{~N}$. Increasing the aeration rate from 30 to $100 \mathrm{~mL} \mathrm{~min}^{-1}$ improved the ammonia recovery, thereby decreasing the energy consumption for recovery by both external power supply (from $16.4 \pm 0.7$ to $2.5 \pm 0.3 \mathrm{kWh} \mathrm{kg}^{-1} \mathrm{~N}$ recovery) and aeration (from $7.7 \pm 0.4$ to $2.0 \pm 0.2 \mathrm{kWh}$ $\mathrm{kg}^{-1} \mathrm{~N}$ recovery). The aeration rate of $300 \mathrm{~mL} \mathrm{~min}^{-1}$ gave higher energy consumption per recovery than that of $100 \mathrm{~mL} \mathrm{~min}{ }^{-1}$, because of similar ammonia recovery but higher aeration energy demand. Other parameters, such as recirculation rate, solution $\mathrm{pH}$, etc., might also affect the energy consumption for ammonia recovery in BES but their contribution could be limited compared to those studied here. 

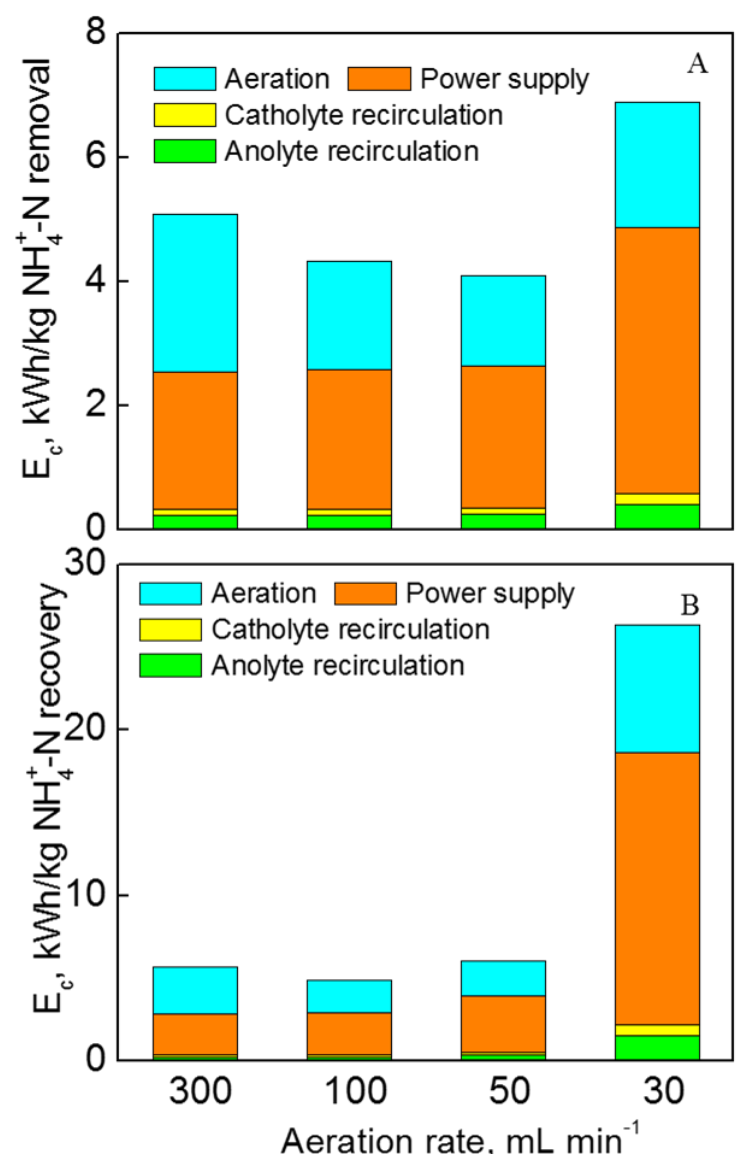

Figure 7.4 Energy consumption by the BES affected by different aeration rate: (A) energy consumption for ammonium removal; and (B) energy consumption for ammonia recovery (blue: aeration; orange: power supply; yellow: catholyte recirculation; green: anolyte recirculation).

\subsubsection{Effects of current generation - external voltage}

The effect of current generation was firstly examined by varying the external voltage from 0 to $0.8 \mathrm{~V}$ across $1 \Omega$ and with $100 \mathrm{~mL} \mathrm{~min}^{-1}$ catholyte aeration. Both current generation and total Coulomb production were strongly affected by the external voltage and showed significant differences $(p<0.05)$. The maximum current density of the BES was $0.3 \pm 0.1,1.1 \pm 0.1$, and $1.6 \pm 0.2 \mathrm{~A} \mathrm{~m}^{-2}$ (Figure 7.5A) while the total Coulomb production was $126 \pm 11,245 \pm 20$, and $329 \pm 33 \mathrm{C}$ (Figure 7.6) with external voltage of $0,0.5$ and $0.8 \mathrm{~V}$, respectively. The COD concentration in the anolyte decreased from $1302 \pm 49 \mathrm{mg} \mathrm{L}^{-1}$ to $277 \pm$ $51 \mathrm{mg} \mathrm{L}^{-1}$, representing COD removal efficiency of $78.8 \pm 3.5 \%$. The ammonium concentration in the 
anolyte decreased from $55 \pm 2 \mathrm{mM}$ to $24 \pm 1 \mathrm{mM}, 21 \pm 1 \mathrm{mM}, 20 \pm 2 \mathrm{mM}$, with external voltage of $0,0.5$ and $0.8 \mathrm{~V}$, respectively, with no significant difference $(p>0.05)$, while the ammonium concentration in the catholyte gradually increased to $16 \mathrm{mM}$ under three tested conditions (Figure 7.5B). The recovery of ammonia gas increased from $2.9 \pm 0.1(0 \mathrm{~V})$ to $7.1 \pm 0.1 \mathrm{~g} \mathrm{~N} \mathrm{~m}^{-2} \mathrm{~d}^{-1}(0.8 \mathrm{~V})$ benefited from higher current generation under a higher external voltage (Figure 7.5C). Mathematical simulation was conducted with the ammonia recovery model containing two inputs: $\mathrm{C} 1$ ) $\mathrm{I}=0.5 \mathrm{~A} \mathrm{~m}^{-2}$ and $\mathrm{t}=48 \mathrm{~h}$, and $\mathrm{C} 2$ ) $\mathrm{I}=0.25 \mathrm{~A} \mathrm{~m}^{-2}$ and $\mathrm{t}=48 \mathrm{~h}$. The simulated results showed that the anolyte ammonium concentration in $\mathrm{C} 1(15 \pm 1 \mathrm{mM})$ was much lower than in $\mathrm{C} 2(31 \pm 1 \mathrm{mM})$ due to the higher current generation in $\mathrm{C}$, which facilitated the ammonium removal from the anolyte (Figure 7.5D). Due to the efficient aeration in the catholyte, the catholyte ammonium concentration was the same at the end of each operating cycle, resulting in higher ammonia recovery in $\mathrm{C} 1$ than $\mathrm{C} 2$ (Figure 7.7).
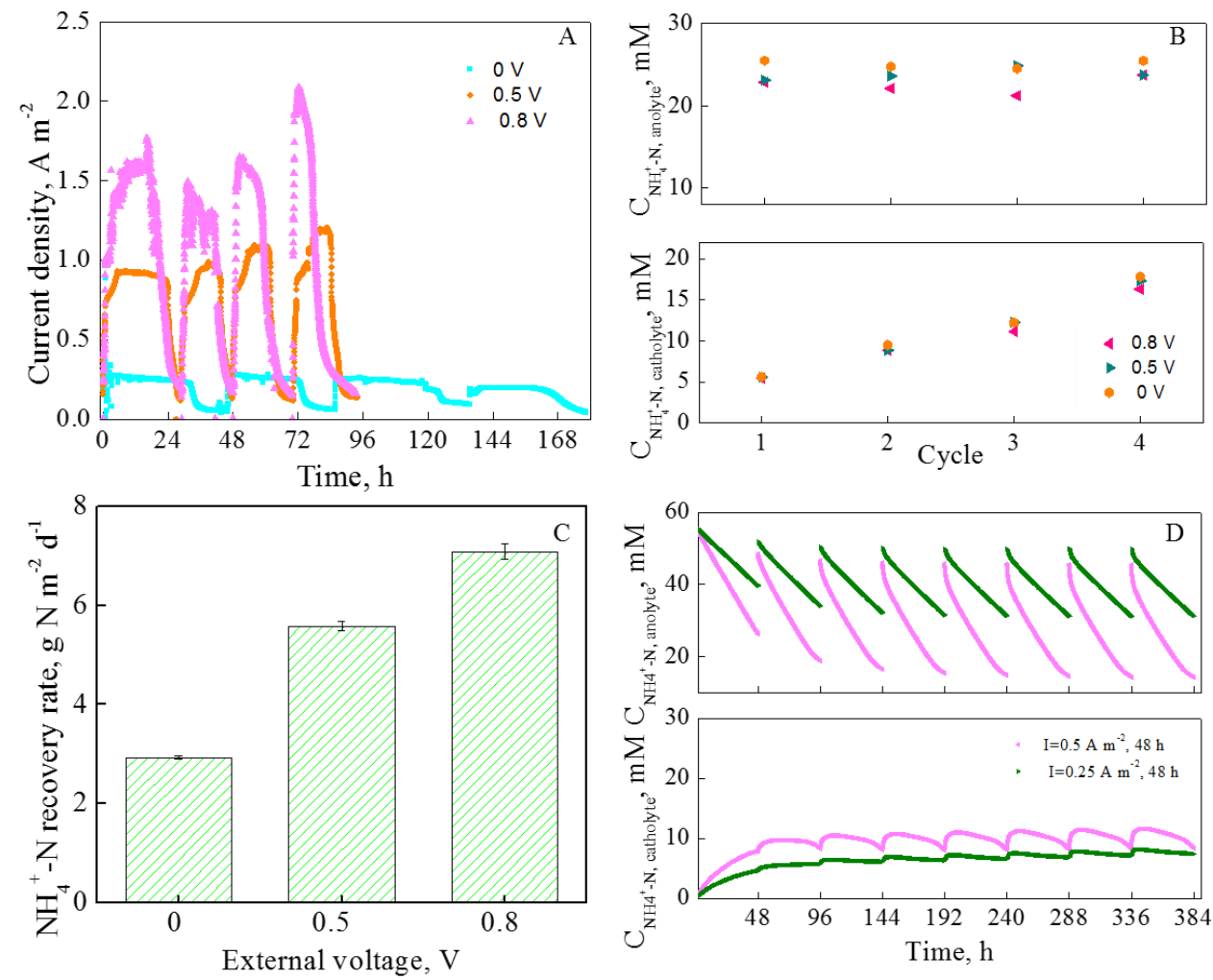

Figure 7.5 Ammonia recovery by the BES affected by different external voltages: (A) current generation; (B) $\mathrm{NH}_{4}{ }^{+}-\mathrm{N}$ concentration in the anolyte and the catholyte effluent; (C) the $\mathrm{NH}_{3}$ recovery rate; and (D) the simulated anolyte and catholyte $\mathrm{NH}_{4}{ }^{+}-\mathrm{N}$ concentrations. The external resistance was $1 \Omega$ and the catholyte aeration rate was $100 \mathrm{~mL} \mathrm{~min}^{-1}$. 


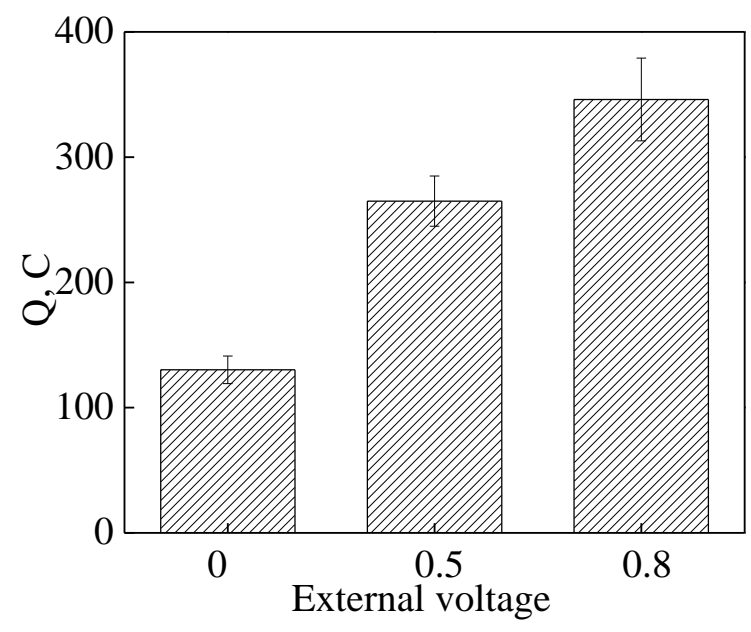

Figure 7.6 The total Coulomb generation per cycle for BES with different external voltage.

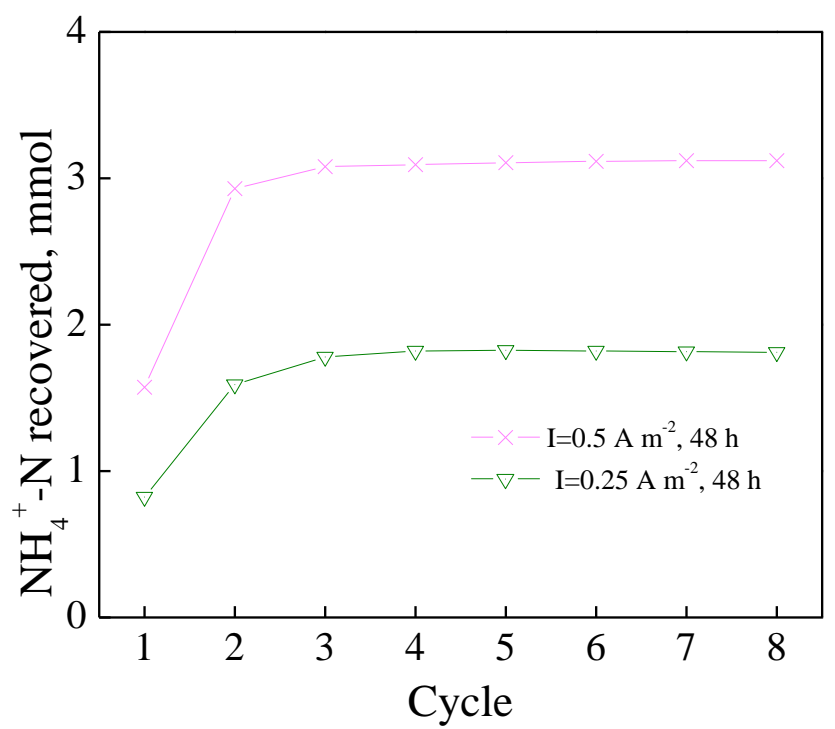

Figure 7.7 The simulated amount of ammonia recovery for BES with different current.

The energy consumption with different external voltages is shown in Figure 7.8. As the external voltage increased from $0 \mathrm{~V}$ to $0.8 \mathrm{~V}$, the energy consumption by power supply increased from 0 to $3.3 \pm 0.3 \mathrm{kWh} \mathrm{kg}^{-1} \mathrm{~N}$ recovery; applying the same aeration rate of $100 \mathrm{~mL} \mathrm{~min}{ }^{-1}$ demanded the same absolute amount of aeration energy, but the normalized energy consumption for recovery by 
aeration decreased from $4.5 \pm 0.4$ to $2.6 \pm 0.3$, because of improved ammonia recovery at a higher external voltage (Figure 7.8). The total energy consumption for recovery decreased from $5.7 \pm 0.5$ to $4.5 \pm 0.6 \mathrm{kWh} \mathrm{kg}^{-1} \mathrm{~N}$ recovery when the external voltage increased from 0 to $0.5 \mathrm{~V}$, and the total energy consumption then increased to $6.4 \pm 0.8 \mathrm{kWh} \mathrm{kg}^{-1} \mathrm{~N}$ recovery with the external voltage of $0.8 \mathrm{~V}$, suggesting that a moderate external voltage could create a balance between increasing ammonia recovery and increased energy consumption by power supply, resulting in a relatively low energy consumption overall.

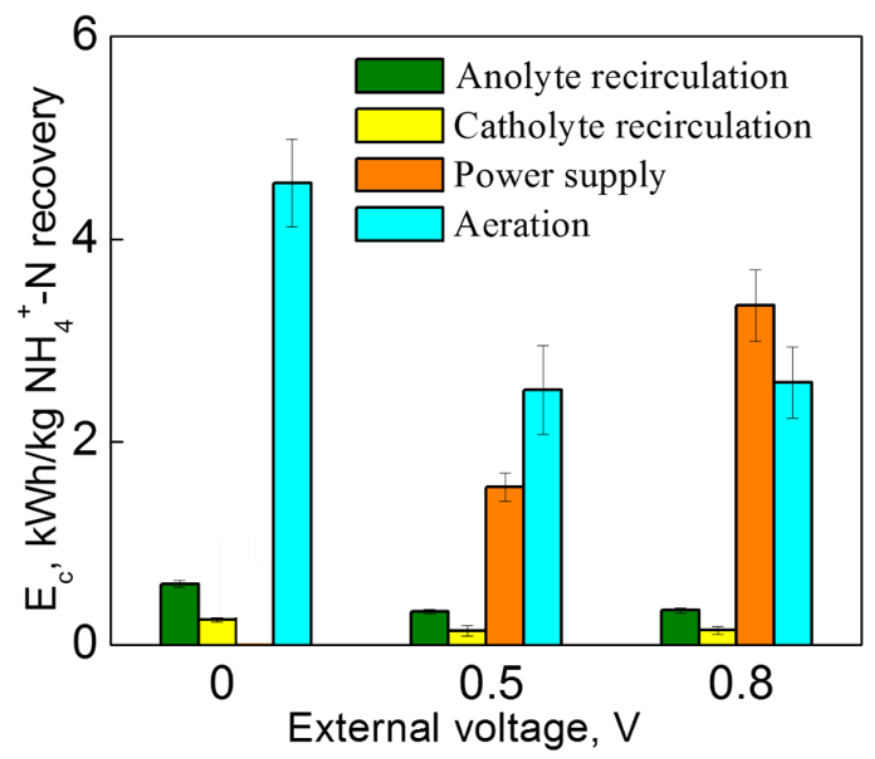

Figure 7.8 Energy consumption for ammonia recovery affected by different external voltages (Green: anolyte recirculation; yellow: catholyte recirculation; orange: power supply; and cyan: aeration).

\subsubsection{Effect of current generation - external resistance}

The effect of current generation was further studied by varying external resistance of $1 \Omega, 10 \Omega$ and $100 \Omega$ under a fixed external voltage of $0.8 \mathrm{~V}$. The maximum current density of the BES increased from $0.8 \pm 0.1$ to $1.6 \pm 0.2 \mathrm{~A} \mathrm{~m}^{-2}$ (Figure 7.9A) when the external resistance decreased from 100 to $1 \Omega$; however, the total 
Coulomb production per cycle did not show significant difference: $352 \pm 22(1 \Omega), 350 \pm 14(10 \Omega)$, and $346 \pm 33 \mathrm{C}(100 \Omega)$ (Figure 7.10) $(p<0.05)$, because of different lengths of batch operation with different external resistance (that is, despite a lower current generation with $100 \Omega$, the BES batch last for a longer period of time, which allowed more Coulombs to be harvested). The ammonium concentration in the anolyte effluent was stable $(22.7 \pm 0.8 \mathrm{mM})$ regardless of the external resistance (Figure 7.11$)$, related to the fact of the similar Coulomb production. The ammonium concentration in the catholyte effluent increased from $5.2 \pm 0.5 \mathrm{mM}$ to $17.3 \pm 0.5 \mathrm{mM}$ from cycle 1 to cycle 4 because of ammonium transport from the anolyte into the catholyte and residue ammonium after stripping; this trend was not significantly different among three tested resistors $(p>0.05)$. The recovery rate of ammonia gas increased from $3.2 \pm 0.1$ to $7.1 \pm 0.1 \mathrm{~g} \mathrm{~N} \mathrm{~m}^{-2} \mathrm{~d}^{-1}$ as the external resistance decreased from $100 \Omega$ to $1 \Omega$, indicating that lower external resistance could accelerate the ammonia recovery within the same time period (Figure 7.9B). The simulation was performed with two inputs: $\mathrm{C} 1$ ) $\mathrm{I}=0.5 \mathrm{~A} \mathrm{~m}^{-2}$ and $\mathrm{t}=48 \mathrm{~h}$, and C3) $\mathrm{I}=1 \mathrm{~A} \mathrm{~m}^{-2}$ and $\mathrm{t}=24$ h. These two simulations ( $\mathrm{C} 1$ and $\mathrm{C} 3)$ represented the current regulation with the same total Coulomb production under different length of the operating period. The simulation showed similar anolyte ammonium concentration at the end of each cycle between $\mathrm{C} 1$ and $\mathrm{C} 3$, confirming that similar Coulomb production resulted in similar amount of removed ammonium from the anolyte (Figure 7.9C). 

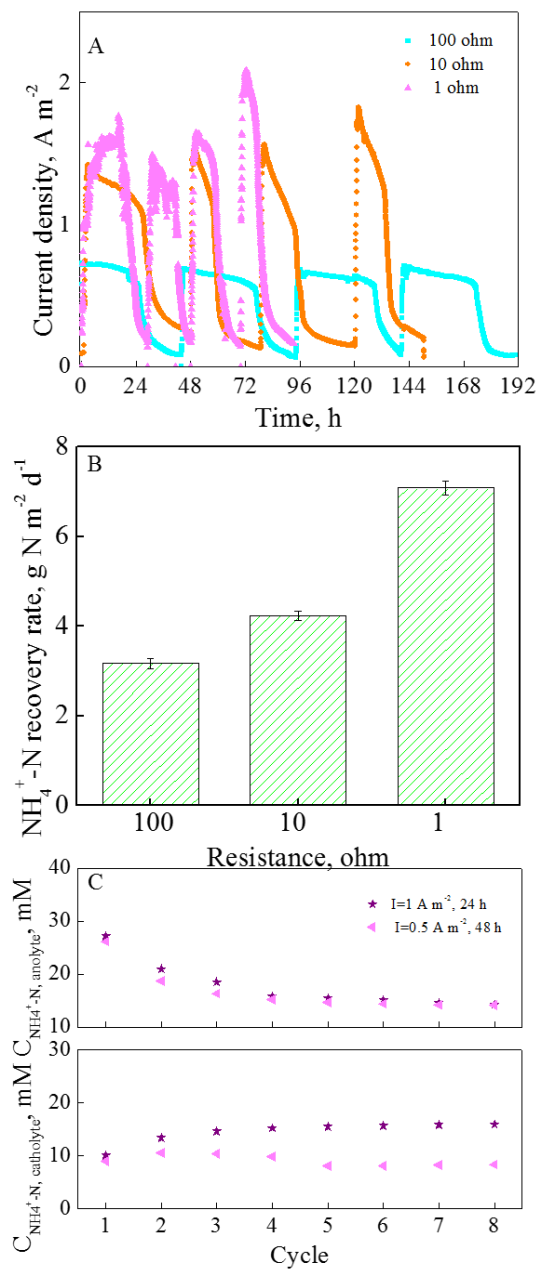

Figure 7.9 The BES performance affected by different external resistances: (A) current generation; (B) the $\mathrm{NH}_{3}$ recovery rate; and (C) simulated $\mathrm{NH}_{4}{ }^{+}-\mathrm{N}$ concentration in the anolyte and catholyte effluents. The external voltage was $0.8 \mathrm{~V}$ and the catholyte aeration rate was $100 \mathrm{~mL} \mathrm{\textrm {min } ^ { - 1 }}$ 


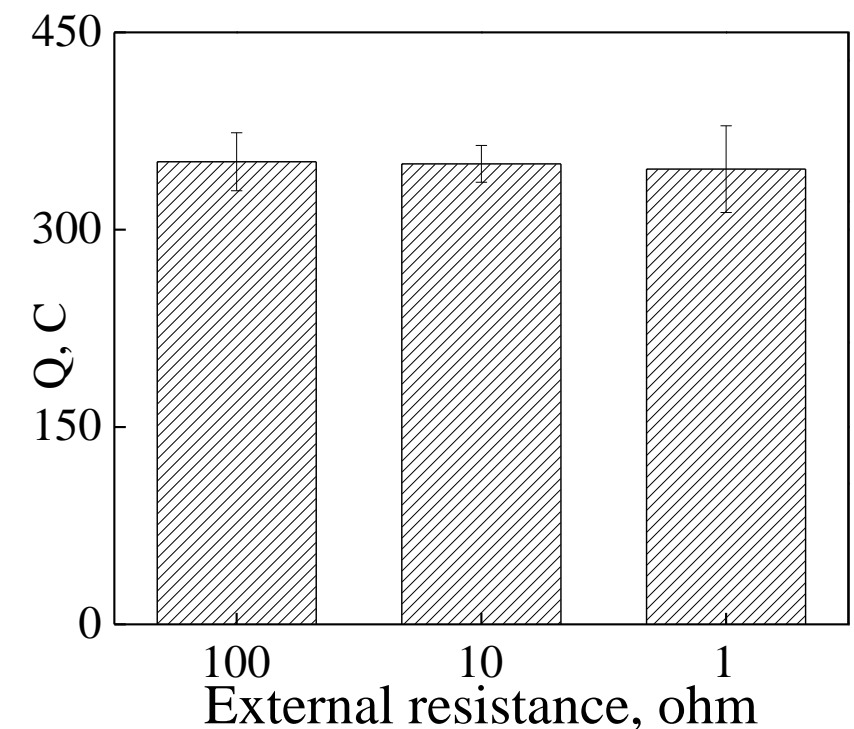

Figure 7.10 The total Coulomb generation per cycle for BES with different external resistance.

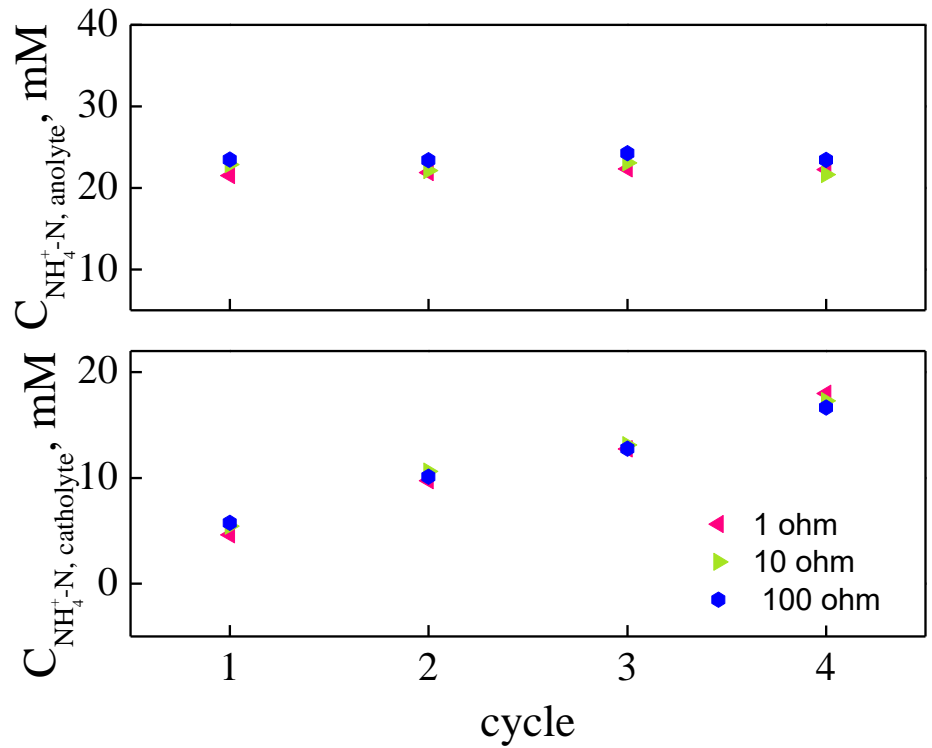

Figure 7.11 The $\mathrm{NH}_{4}{ }^{+}-\mathrm{N}$ concentration for anolyte and catholyte effluent in BES with different external resistances.

Energy consumption was $7.2 \pm 0.7,6.6 \pm 0.7$, and $5.7 \pm 0.5 \mathrm{kWh} \mathrm{kg}^{-1} \mathrm{~N}$ when the external resistance was $100 \Omega, 10 \Omega$ and $1 \Omega$, respectively (Figure 7.12). As the external resistance 
decreased from $100 \Omega$ to $1 \Omega$, the energy consumption by the catholyte aeration decreased from $4.4 \pm 0.4$ to $2.3 \pm 0.3 \mathrm{kWh} \mathrm{kg}^{-1} \mathrm{~N}$ recovery, resulting in the decrease of total energy consumption per kg $\mathrm{N}$ recovery. The energy consumption by power supply did not show significant difference regardless of the external resistances $\left(2.9 \pm 0.3 \mathrm{kWh} \mathrm{kg}^{-1} \mathrm{~N}\right.$ recovery), resulting from the similar total Coulomb generation. As the external resistance increased from $1 \Omega$ to $100 \Omega$, the power generation in BES increased from 0.02 to $0.9 \pm 0.1 \mathrm{kWh} \mathrm{kg}^{-1} \mathrm{~N}$ recovery. The internal resistance for the BES was estimated to be $315 \Omega$ according to the polarization curve shown in Figure 7.13 and thus external resistance such as $100 \Omega$ that was close to the internal resistance of the BES would result in more energy production.

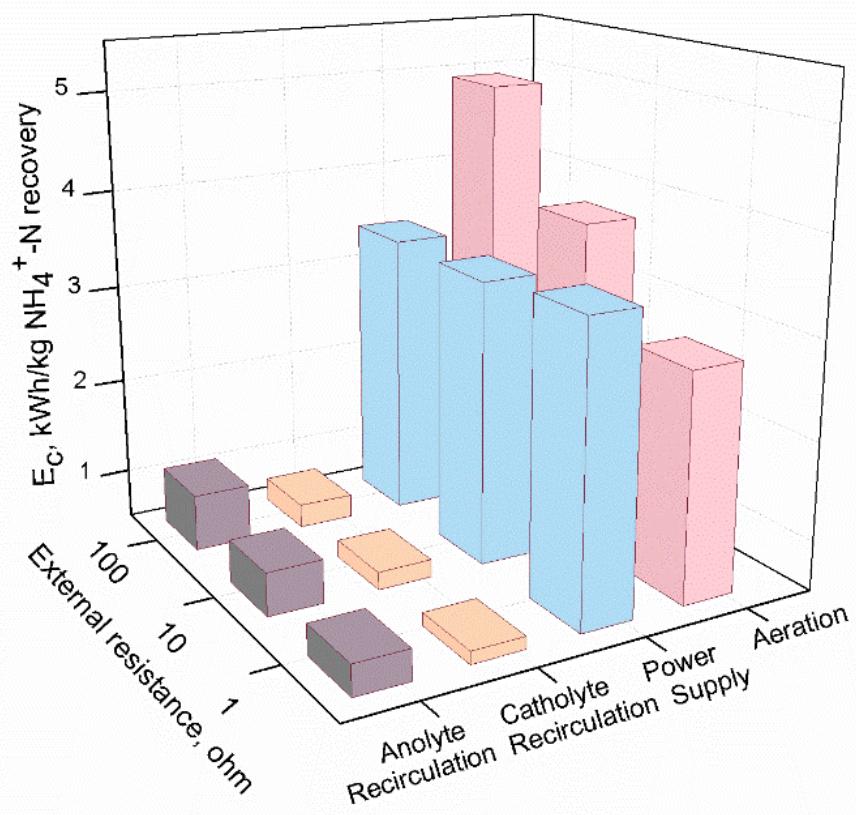

Figure 7.12 Energy consumption for ammonia recovery affected by different external resistances (Gray: anolyte recirculation; yellow: catholyte recirculation; cyan: power supply; and magenta: aeration). 


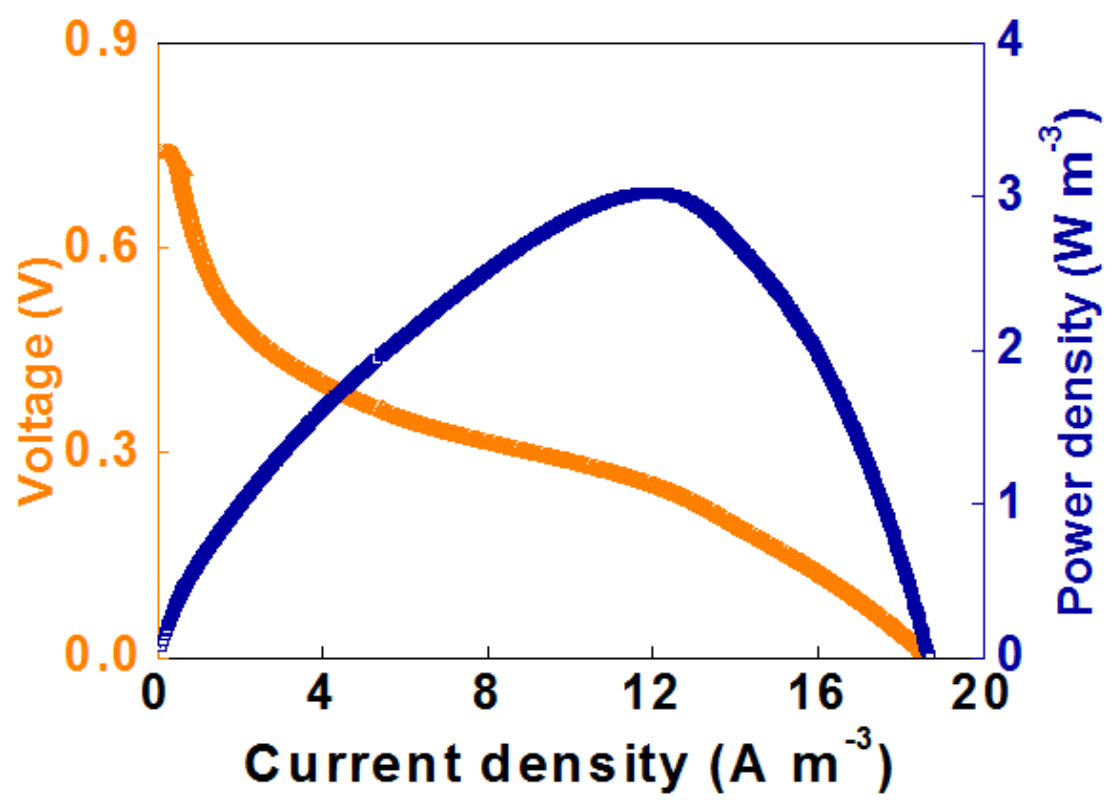

Figure 7.13 The polarization curve for BES.

\subsubsection{Perspectives}

The present BES achieved the lowest energy consumption of $4.5 \pm 0.6 \mathrm{kWh} \mathrm{kg}^{-1} \mathrm{~N}$ for ammonia recovery with a recovery rate of $5.6 \mathrm{~g} \mathrm{~N} \mathrm{~m}^{-2} \mathrm{~d}^{-1}$, under the condition of $100 \mathrm{~mL} \mathrm{~min}^{-1}$ catholyte aeration, $0.5 \mathrm{~V}$ external voltage and $1 \Omega$ resistance. This energy consumption is much lower than that of the Haber-Bosch process for ammonia synthesis (8-14 kWh per kg N) (Erisman et al. 2008, Maurer et al. 2003b), and is also low when comparing to the existing nitrogen removal/recovery technologies (Table 1). The air stripping process requires $11-14 \mathrm{kWh} \mathrm{kg}^{-1} \mathrm{~N}$ for nitrogen removal and potential recovery, including energy consumption for chemical production, aeration pumping and excess heat. Biological nitrogen removal processes such as combined nitrification and denitrification, and anammox convert ammonia to nitrogen gas. Combined nitrification and denitrification requires $11-14 \mathrm{kWh} \mathrm{kg}^{-1} \mathrm{~N}$ while anammox needs $1-5 \mathrm{kWh} \mathrm{kg}^{-1} \mathrm{~N}$ for ammonia removal. Although anammox has a low energy demand, it removes ammonia from wastewater 
instead of recovery. Therefore, BES, which enables ammonia recovery and has relatively low energy consumption, will be of strong interest to sustainable nitrogen management in wastewater treatment. However, the recovery rate of ammonia in BES $\left(0.40 \mathrm{~kg} \mathrm{~N} \mathrm{~m}^{-3} \mathrm{~d}^{-1}, \mathrm{~kg} \mathrm{~N}\right.$ per treated water volume per day) is not high, particularly comparing to that of anammox $\left(0.27-2.33 \mathrm{~kg} \mathrm{~N} \mathrm{~m}^{-}\right.$

${ }^{3} \mathrm{~d}^{-1}$ ) (Table 7.2). The ammonia removal/recovery rate was represented with unit $\mathrm{kg} \mathrm{N} \mathrm{m}^{-3} \mathrm{~d}^{-1}$, because the existing technologies are not membrane based. The recovery rate of ammonia in BES is comparable with that in air stripping $\left(0.10-0.76 \mathrm{~kg} \mathrm{~N} \mathrm{~m}^{-3} \mathrm{~d}^{-1}\right)$ and combined nitrification and denitrification (0.14-0.58 $\left.\mathrm{kg} \mathrm{N} \mathrm{m}^{-3} \mathrm{~d}^{-1}\right)$, while the energy consumption is much lower in BES. The ammonia recovery in BES is affected by ammonia input, current generation (which is related to organic compounds, microbial activities, and operating temperature), and aeration, which should be optimized to enhance the recovery rate.

Table 7.2 Comparison of different technologies for ammonia removal and recovery from wastewater

\begin{tabular}{lllllll}
\hline Technology & Purpose & Rate & Efficiency & Energy demand & References \\
\cline { 2 - 6 } & & $\mathrm{kg} \mathrm{m}^{-3} \mathrm{~d}^{-1}$ & & $(\mathrm{kWh}$ per kg N) & \\
\hline Air stripping & Recovery & $0.10-0.76$ & $91 \%$ & $11-14$ & $\begin{array}{l}\text { (Maurer et } \\
2003 \mathrm{~b})\end{array}$ \\
$\begin{array}{l}\text { Nitrification } \\
\text { denitrification }\end{array}$ & Removal & $0.14-0.58$ & $90-98 \%$ & $11-14$ & (Won et al. 2015) \\
Anammox & Removal & $0.27-2.33$ & $88-99 \%$ & $1-5$ & (Lackner et al. \\
BES & Recovery & 0.40 & $83 \%$ & 4.5 & 2014) & This study \\
BES & Removal & 0.48 & $87 \%$ & 2.2 & This study \\
\hline
\end{tabular}


Understanding energy consumption will help to establish an energy balance, which can show us how far we are from an energy-neutral (or energy-positive) ammonia recovery process by using BES. Because high current generation could facilitate ammonium transport and subsequent ammonia recovery, an external resistor at extremely low value of $1 \Omega$ was used in the condition that had the lowest energy consumption for recovery. With such low external resistance, power output can be neglected and thus there would be little energy production. A previous study of ammonia recovery in an MFC achieved a surplus energy of $1.0 \mathrm{kWh} \mathrm{kg}^{-1} \mathrm{~N}$, in which more energy was produced than needed for the ammonia recovery, resulting from a small catholyte volume for aeration (aeration rate: $0.5 \mathrm{vvm}$; catholyte volume: $20 \mathrm{~mL}$ ) and no external power supply (Kuntke et al. 2012). However, the ammonia recovery rate in that study was relatively low at $3.3 \mathrm{~g} \mathrm{~N} \mathrm{~m}^{-2}$ $\mathrm{d}^{-1}$, less than half of the highest ammonia recovery rate of $7.1 \mathrm{~g} \mathrm{~N} \mathrm{~m}^{-2} \mathrm{~d}^{-1}$ obtained in this study (with an aeration rate of $1.7 \mathrm{vvm}$ and external supply of $0.8 \mathrm{~V}$ ). Therefore, there is a trade-off between the increased ammonia recovery rate and increased energy consumption and the benefits of more ammonia recovery at a price of higher energy consumption will require further analysis.

Reducing energy consumption and considering energy production by BES warrant further investigation through optimized configuration design and operation. Decreasing the catholyte volume could be one of the possible approaches to reduce energy consumption. With a smaller catholyte volume, the energy consumed by aeration could be reduced with a more efficient ammonia recovery from the catholyte. To produce energy, the BES can be operated in either an MFC mode (for electricity generation) or an MEC mode (for hydrogen production). The MEC mode may be preferred because of higher current generation under an external voltage and thus a higher ammonia recovery rate. With an external voltage $>0.2 \mathrm{~V}$ and in the absence of aeration, 
hydrogen gas can be produced in the cathode (Ki et al. 2016, Liu et al. 2005). Although an external voltage was also applied to the BES in this study, the use of oxygen as a terminal electron acceptor prevented hydrogen production. Thus, future studies may consider a cathode without air aeration. However, our study has demonstrated the importance of aeration to ammonia stripping and thus recovery. To actively drive ammonia out of the catholyte in the absence of air aeration, recirculation of the produced hydrogen gas could be considered.

\subsection{Conclusions}

This study has quantified the energy consumption for ammonia recovery affected by catholyte aeration rates and current generation in a BES through integrated experiments and mathematical modeling. The aeration rate in the catholyte could strongly impact the ammonia recovery and energy consumption: a high aeration rate resulted in a high ammonia recovery while a moderate aeration rate $\left(100 \mathrm{~mL} \mathrm{~min}{ }^{-1}\right)$ led to the lowest energy consumption, indicating that there is a trade-off between the increased ammonia recovery rate and increased energy consumption. The high current generation, which was achieved with either high external voltage $(0.8 \mathrm{~V})$ or low external resistance $(1 \Omega)$, could improve the ammonia recovery. However, a trade-off existed between energy consumption and ammonia recovery. Compared to other ammonia removal/recovery technologies such as combined nitrification and denitrification, anammox, and air stripping, BES accomplishes ammonia recovery, in addition to ammonia removal, and has relatively low energy consumption. These advantages make BES a promising technology for energy-efficient resource recovery from wastewater. 


\title{
Chapter 8 Self-supplied ammonium bicarbonate draw solute for achieving wastewater treatment and recovery in a microbial electrolysis cell-forward osmosis-coupled system
}

(This section has been published as Qin, M., \& He, Z.* (2014). Self-supplied ammonium bicarbonate draw solute for achieving wastewater treatment and recovery in a microbial electrolysis cell-forward osmosis-coupled system. Environmental Science \& Technology Letters, 1(10), 437-441.)

\begin{abstract}
This study has presented a proof of concept system for self-sustained supply of ammonium-based draw solute for wastewater treatment, through coupling a microbial electrolysis cell (MEC) and a forward osmosis (FO). The MEC produced an ammonium bicarbonate draw solute via recovering ammonia from synthetic organic solution, which was then applied in the FO for extracting water from the MEC anode effluent. The recovered ammonium could reach a concentration of 0.86 mol $\mathrm{L}^{-1}$, and with this draw solution, the FO extracted $50.1 \pm 1.7 \%$ of the MEC anode effluent. The lost ammonium during heat regeneration could be supplemented with additional recovered ammonium in the MEC. The MEC achieved continuing treatment of both organic and ammonium in the returned feed solution mixed with fresh anolyte, though at lower efficiency compared to that with completely fresh anolyte. These results encourage further investigation to optimize the coordination between MEC and FO with improved performance.
\end{abstract}




\subsection{Introduction}

Wastewater reuse is critical to sustainable wastewater treatment and requires effective technologies to extract high-quality water from wastewater (Sharma and Sanghi 2013). Membrane-based processes have been studied and/or employed to produce high-quality water from wastewater (Logan and Elimelech 2012). Among them, forward osmosis (FO) process is of particular interest because of its low energy consumption and reduced membrane fouling (Lutchmiah et al. 2014a). In an FO process, water moves across a semipermeable membrane from high water potential to low water potential, driven by an osmotic pressure gradient (Zhang et al. 2011a).

Draw solute plays a key role in creating an osmotic gradient for water extraction in FO (Lutchmiah et al. 2014a). Because permeated water can dilute draw solution and some draw solutes may be lost due to reverse salt flux, draw solute needs to be recovered by additional processes or supplemented with fresh draw solute, thereby increasing energy requirement and operating cost of the FO (Achilli et al. 2010). Various types of draw solutes have been proposed (Klaysom et al. 2013), and among them, thermolytic ammonium salts, such as ammonium bicarbonate, have been demonstrated as an efficient and recyclable draw solute because of their high solubility, osmotic efficiency, and circulation capacity (McCutcheon et al. 2005). More importantly, ammonium bicarbonate can be recovered using moderate heat, which has significant energy advantages compared with regeneration of other draw solutes (McGinnis et al. 2007, Trypuć and Kiełkowska 1998). However, the application of ammonium bicarbonate as a draw solute has been limited because of high reverse salt flux that causes the loss to the feed solution, which will not only 
require additional supply of fresh draw solute, but also deteriorate the quality of the remaining feed solution (Chekli et al. 2012).

The concentration of ammonium in some wastewaters such as digester centrate and landfill leachate can be over $500 \mathrm{mg} \mathrm{L}^{-1}$ (Renou et al. 2008). Thus, recovering ammonia from wastewater may provide a sustainable approach for supplying draw solute to FO treatment, which is then used to treat the same wastewater. An innovative method for ammonium recovery from wastewater is using bioelectrochemical systems (BES) (Kelly and He 2014). During electricity generation, ammonium migrates from the anode (wastewater) into the cathode through a cation exchange membrane. It was found that high current generation could promote ammonium movement and high $\mathrm{pH}$ of the catholyte facilitates the conversion of ammonium into ammonia gas, which can be stripped out of the catholyte and then recovered. To make a draw solute, carbon dioxide will be needed. The anode reaction releases carbon dioxide; additional carbon dioxide may be supplied by combustion processes, for example, a power plant that provides both carbon dioxide and low heat for recovering ammonium bicarbonate (Cusick et al. 2012).

Herein, we investigated the feasibility of the above concept with a coupled system consisting of a microbial electrolysis cell (MEC) and an FO: the MEC was to degrade organic compounds (in its anode) and recover ammonia (in the cathode) for preparing draw solute for the following FO treatment, which extracted clean water from the MEC anode effluent. The key parameters including ammonia recovery and regeneration, organic degradation, electricity generation, and water flux were investigated. 


\subsection{Materials and Methods}

\subsubsection{The MEC-FO system}

A bench-scale MEC-FO system consisted of two units, an MEC unit and an FO unit, which were linked through two liquid streams (Figure 8.1). The synthetic organic solution was fed into the anode chamber of the MEC, and its effluent then flowed into the feed side of the FO. The gas streams from the anode and the cathode of the MEC were channeled through two 100-mL glass bottles. The first bottle was an absorption bottle, filled with deionized water to collect gaseous ammonia $\left(\mathrm{NH}_{3}\right)$ and $\mathrm{CO}_{2}$ to generate ammonium bicarbonate. Additional carbon dioxide was introduced into this bottle at a flow rate of $1.26 \mathrm{~mL} \mathrm{~min}^{-1}$ by a $\mathrm{CO}_{2}$ cylinder. The second bottle was filled with $1 \mathrm{M}$ sulphuric acid $\left(\mathrm{H}_{2} \mathrm{SO}_{4}\right)$ to absorb the excessive $\mathrm{NH}_{3}$ that could not be captured by the first bottle, named "recovery bottle". Once the concentration of ammonium ions in the absorption bottle reached $0.80 \mathrm{M}$, the solution was transferred to the FO as the draw solution for water extraction from the treated organic solution (from the MEC anode). When the water flux in the FO dropped below $1.0 \mathrm{~L} \mathrm{~m}^{-2} \mathrm{~h}^{-1}(\mathrm{LMH})$, the diluted draw solution was sent to a heat recovery unit, in which the temperature was set at $80^{\circ} \mathrm{C}$. The $\mathrm{NH}_{3}$ and $\mathrm{CO}_{2}$ were separated from the draw solution and flowed into an adsorption flask containing deionized water to regenerate the draw solution. The concentrated feed solution from the FO was adjusted to $\mathrm{pH} 7.0$ with $80 \mu \mathrm{L}$ of $0.1 \mathrm{M}$ $\mathrm{NaOH}$ solution and returned to the MEC for further treatment. 


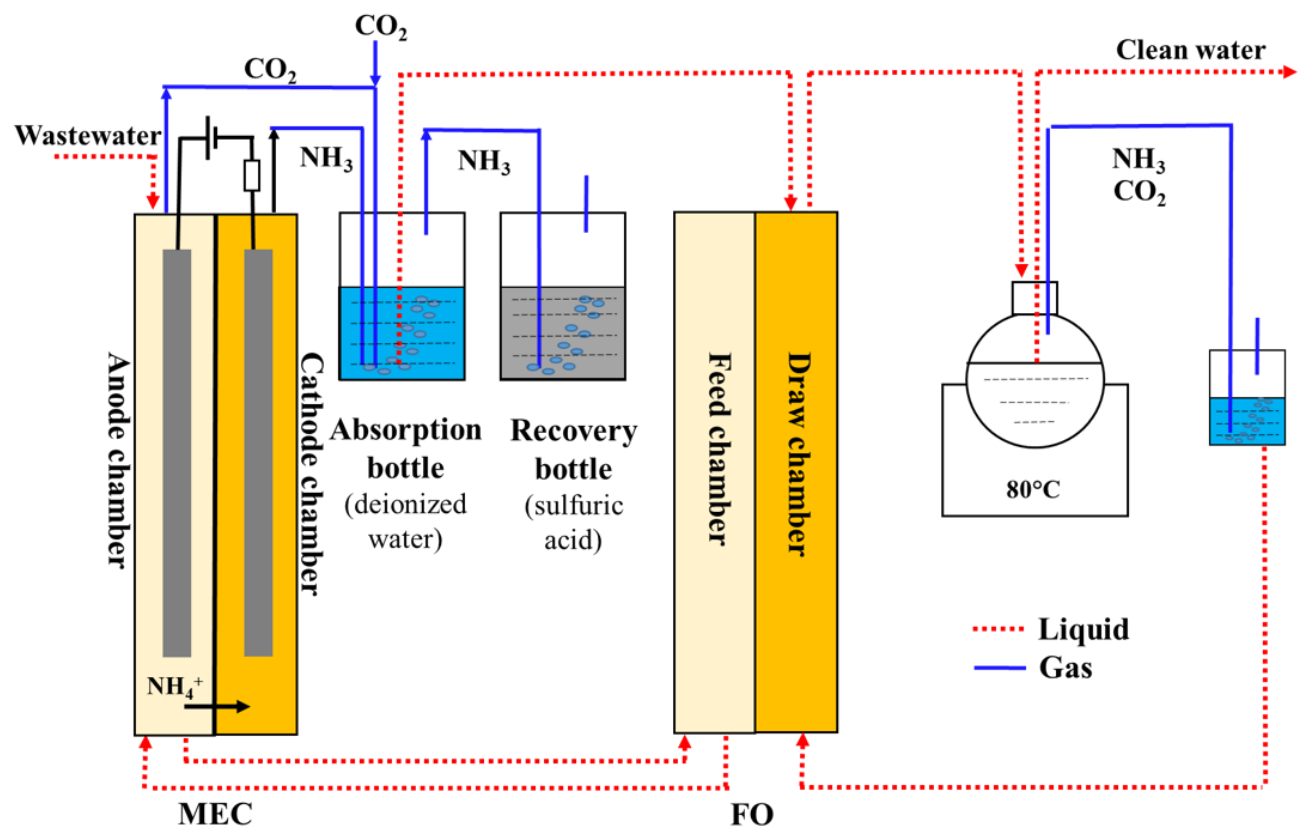

Figure 8.1 The schematic design of the MEC-FO coupled system

\subsubsection{MEC and FO setup and operation}

A tubular MEC was constructed with more details in the Supporting Information (SI). A 1-m long carbon brush was folded to fit into the anode compartment as the anode electrode. The cathode electrode was a piece of carbon cloth $\left(160 \mathrm{~cm}^{2}\right)$ coated with platinum/carbon as a catalyst $(0.3 \mathrm{mg}$ $\mathrm{Pt} \mathrm{cm}^{-2}$ ) (Zhang et al. 2010). The cathode compartment was aerated to provide oxygen for reaction and to strip ammonia from the catholyte (Liao et al. 1995). The synthetic organic solution that mimicked piggery wastewater or its anaerobic digestion effluent(Lei et al. 2007, Sánchez et al. 1995) contained (per liter of deionized water): glucose, 3.0 g; $\mathrm{NH}_{4} \mathrm{Cl}, 3.0 \mathrm{~g} ; \mathrm{NaHCO}_{3}, 5.0 \mathrm{~g} ; \mathrm{NaCl}$, $0.5 \mathrm{~g} ; \mathrm{MgSO}_{4}, 0.015 \mathrm{~g} ; \mathrm{CaCl}_{2}, 0.02 \mathrm{~g}$; and trace elements, $1 \mathrm{~mL}$ (Angenent and Sung 2001). An external voltage of $0.8 \mathrm{~V}$ was applied to the circuit by a power supply (CSI3644A, Circuit Specialists, Inc., Mesa, AZ, USA) according to a previous study (Yossan et al. 2013). 
The MEC had a tubular configuration, which used a CEM tube (Membrane International Inc., Ringwood, NJ, USA) to separate the anode and the cathode. The membrane tube was installed in a glass tube, thereby creating two compartments, the cathode (inside the membrane tube) and the anode (between the membrane and the glass tube). The liquid volume of the anode compartment was $500 \mathrm{~mL}$, and the cathode contained $500 \mathrm{~mL}$ deionized water. The anode was inoculated with the anaerobic sludge from the Peppers Ferry Regional Wastewater Treatment Plant (Radford, VA, USA).

The MEC was operated in a batch mode at room temperature $\left(\sim 20^{\circ} \mathrm{C}\right)$. The anolyte was partially replaced (50\%) every $48 \mathrm{~h}$ while the catholyte was not changed during the entire experiment. When the MEC was linked to the FO through supplying/receiving the feeding solution, $250 \mathrm{~mL}$ of the fresh anolyte was added to the MEC in each cycle (while the other $250 \mathrm{~mL}$ anolyte was the concentrated feed solution from the FO).

A SEPA CF Cell (Sterlitech Corporation, Kent, WA, USA) was used as a FO cell. The FO membrane was cellulose triacetate (Hydration Technologies Inc., Albany, OR, USA), and installed with its active layer facing the feed solution. During the operation, both the feed solution and the draw solution were recirculated at $96 \mathrm{~mL} \mathrm{~min}^{-1}$. The water flux (LMH) is calculated by the change of weight of the feed or draw solution (Wang et al. 2010a). The reverse salt flux ( $\mathrm{g} \mathrm{m}^{-2} \mathrm{~h}^{-1}, \mathrm{gMH}$ ) from the draw side to the feed side was calculated by measuring the salt concentration change in feed solution (Wang et al. 2010a). 


\subsubsection{Measurement and analysis}

The voltage across the external resistance in the MEC circuit was recorded every 5 min by a digital multimeter (Keithley Instruments Inc., Cleveland, OH, USA). The $\mathrm{pH}$ of liquid stream was measured by a benchtop $\mathrm{pH}$ meter (Oakton Instruments, Vernon Hills, IL, USA). Conductivity was measured with a benchtop conductivity meter (Mettler-Toledo, Columbus, OH, USA). The concentration of chemical oxygen demand (COD) was measured using a colorimeter (Hach DR/890, Hach Company, Loveland, CO, USA). The concentration of ammonia was measured using an ISE meter (Fisher Scientific, Pittsburgh, PA, USA). The $\mathrm{CO}_{2}$ was analyzed using a Shimadzu GC-14A gas chromatograph equipped with a thermal conductivity detector (TCD). Water flux in the FO was calculated by the change of weight recorded on a balance (Scort Pro, Ohous, Columbia, MD, USA).

\subsection{Results and Discussion}

Preparing draw solution and generating feed solution was examined in the MEC via investigating the ammonia recovery and the treatment of synthetic organic solution. Under an applied voltage of $0.8 \mathrm{~V}$, the MEC produced $1.8 \pm 0.1 \mathrm{~A} \mathrm{~m}^{-2}$ (Figure 8.2A), and decreased the COD concentration from $3.23 \pm 0.16$ to $1.27 \pm 0.08 \mathrm{~g} \mathrm{~L}^{-1}$, representing a COD removal efficiency of $60.6 \pm 2.2 \%(0.98$ $\left.\pm 0.07 \mathrm{~kg} \mathrm{~m}^{-3} \mathrm{~d}^{-1}\right)($ Figure 8.2B).

The ammonium concentration in the anolyte decreased from $0.92 \pm 0.08$ to $0.17 \pm 0.05 \mathrm{~g} \mathrm{NH}_{4}{ }^{+}-\mathrm{N}$ $\mathrm{L}^{-1}$, representing removal of $81.5 \pm 4.4 \%\left(0.49 \pm 0.04 \mathrm{~kg} \mathrm{~m}^{-3} \mathrm{~d}^{-1}\right)$ (Figure 8.2C). The catholyte had an $\mathrm{NH}_{4}{ }^{+}-\mathrm{N}$ concentration of $0.47 \pm 0.12 \mathrm{~g} \mathrm{~L}^{-1}$. Assuming one electron moves one ammonium 
ion from the anode chamber into the cathode chamber, migration of ammonium ions could contribute to $48.9 \%$ of ionic flux through the CEM, indicating that ammonium ions acted as a proton shuttle in the MEC system (Cord-Ruwisch et al. 2011). The catholyte $\mathrm{pH}$ increased from 6.89 to 9.33 (Figure 8.2D). The aeration in the cathode compartment promoted the stripping of ammonia out of the catholyte (Kim et al. 2008). Although the catholyte $\mathrm{pH}$ of the present MEC was lower than that of an MEC without aeration (up to 13 (Zhang et al. 2014b)), the lower catholyte $\mathrm{pH}$ could decrease the cell overpotential and improve system stability (Zhuang et al. 2010). The stripped ammonia was collected in the absorption bottle, in which the ammonium concentration increased gradually to $11.4 \mathrm{~g} \mathrm{~L}^{-1}: 45.3 \%$ of input ammonium was recovered by deionized water in the absorption bottle, while $26.1 \%$ was collected by sulfuric acid in the recovery bottle. The gas analysis shows that $87.3 \pm 3.4 \%$ of gas generated from the anode was $\mathrm{CO}_{2}$. The volume of generated $\mathrm{CO}_{2}$ in one batch was about $47 \mathrm{~mL}$, which was not enough for generating ammonium
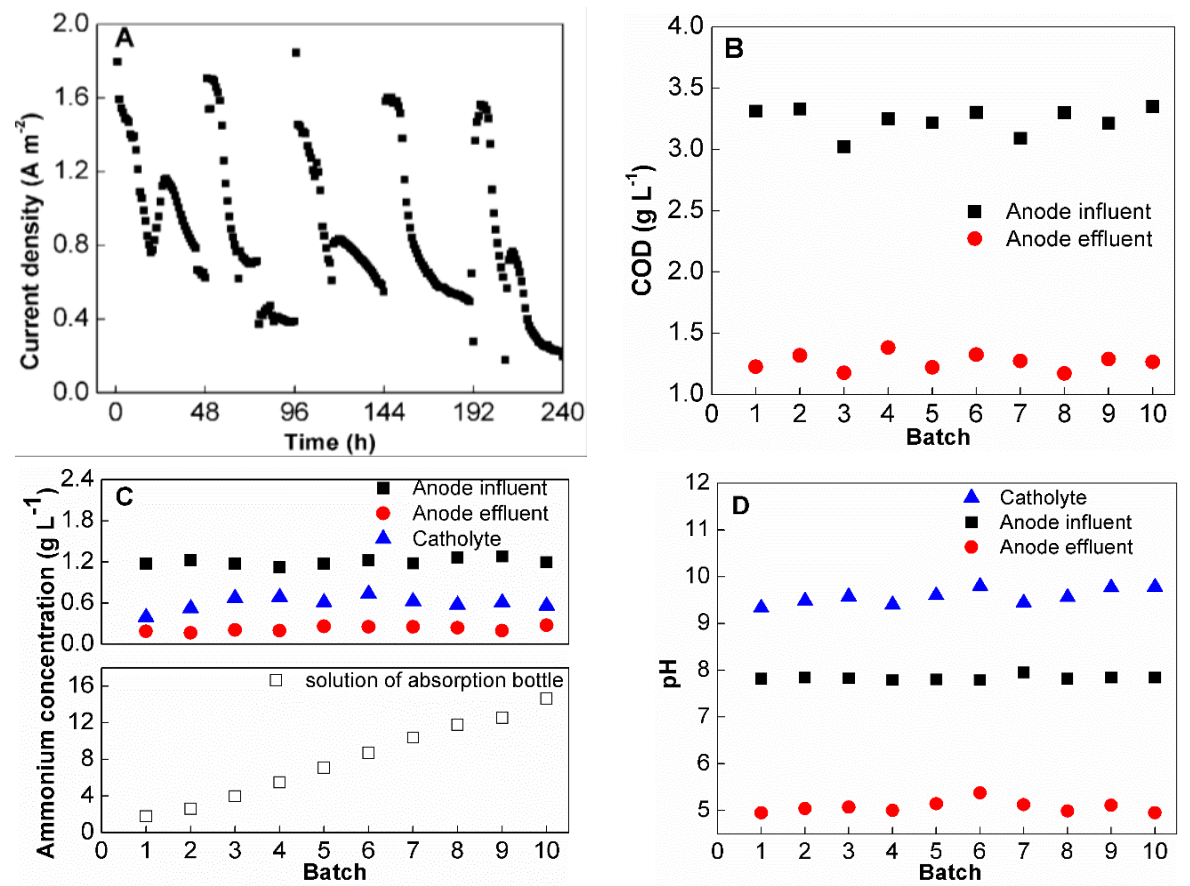

Figure 8.2 Ammonia recovery in the MEC: (A) current density, (B) COD concentration, $(C)$ ammonium nitrogen concentration in the MEC and the solution of absorption bottle, and (D) the $\mathrm{pH}$ in each chamber. 
bicarbonate (considering the ratio between ammonium and carbon dioxide). Thus, additional $\mathrm{CO}_{2}$ was added into the adsorption bottle. After 10 batches, the $\mathrm{NH}_{4}{ }^{+}-\mathrm{N}$ concentration reached $11.4 \mathrm{~g}$ $\mathrm{L}^{-1}$ (Figure 8.2 Ammonia recovery in the MEC: (A) current density, (B) COD concentration, (C) ammonium nitrogen concentration in the MEC and the solution of absorption bottle, and (D) the $\mathrm{pH}$ in each chamber.C), representing $0.86 \mathrm{~mol} \mathrm{~L}^{-1}$ ammonium bicarbonate.

The generated ammonium bicarbonate solution was transferred to the FO as a draw solution for water recovery from the MEC anode effluent. The results demonstrated the effectiveness of this draw solution. The FO achieved a maximum water flux of $2.9 \pm 0.1 \mathrm{LMH}$, within the range of the FO processes using ammonium bicarbonate as a draw solution in other studies (Chanukya et al. 2013, McCutcheon et al. 2005). The water flux decreased to $0.8 \pm 0.1 \mathrm{LMH}$ after 5-h operation (Figure 8.3A), and $50.1 \pm 1.7 \%$ of the MEC anode effluent was extracted into the draw side. After the FO treatment, the ammonium concentration in the feed solution increased from $0.24 \pm 0.03$ to $1.66 \pm 0.15 \mathrm{~g} \mathrm{~L}^{-1}$, likely because of the concentrating effect due to reduced liquid volume and ammonium intrusion from the draw side via reverse salt flux. It was determined that the reverse salt flux was $2.6 \pm 0.4 \mathrm{gMH}$ (Figure 8.3B), with a salt rejection of $87.9 \pm 1.8 \%$. During regeneration of the draw solution in the heat recovery unit, $83.1 \%$ of ammonium was recovered at $80{ }^{\circ} \mathrm{C}$, and the lost part was supplied by the additionally recovered ammonia in the MEC. The lower recovery temperature at $60{ }^{\circ} \mathrm{C}$ achieved only $51.1 \%$ of ammonium recovery. After heat regeneration, the extracted water contained less than $1 \mathrm{mg} \mathrm{L}^{-1}$ of ammonium and undetected COD. 

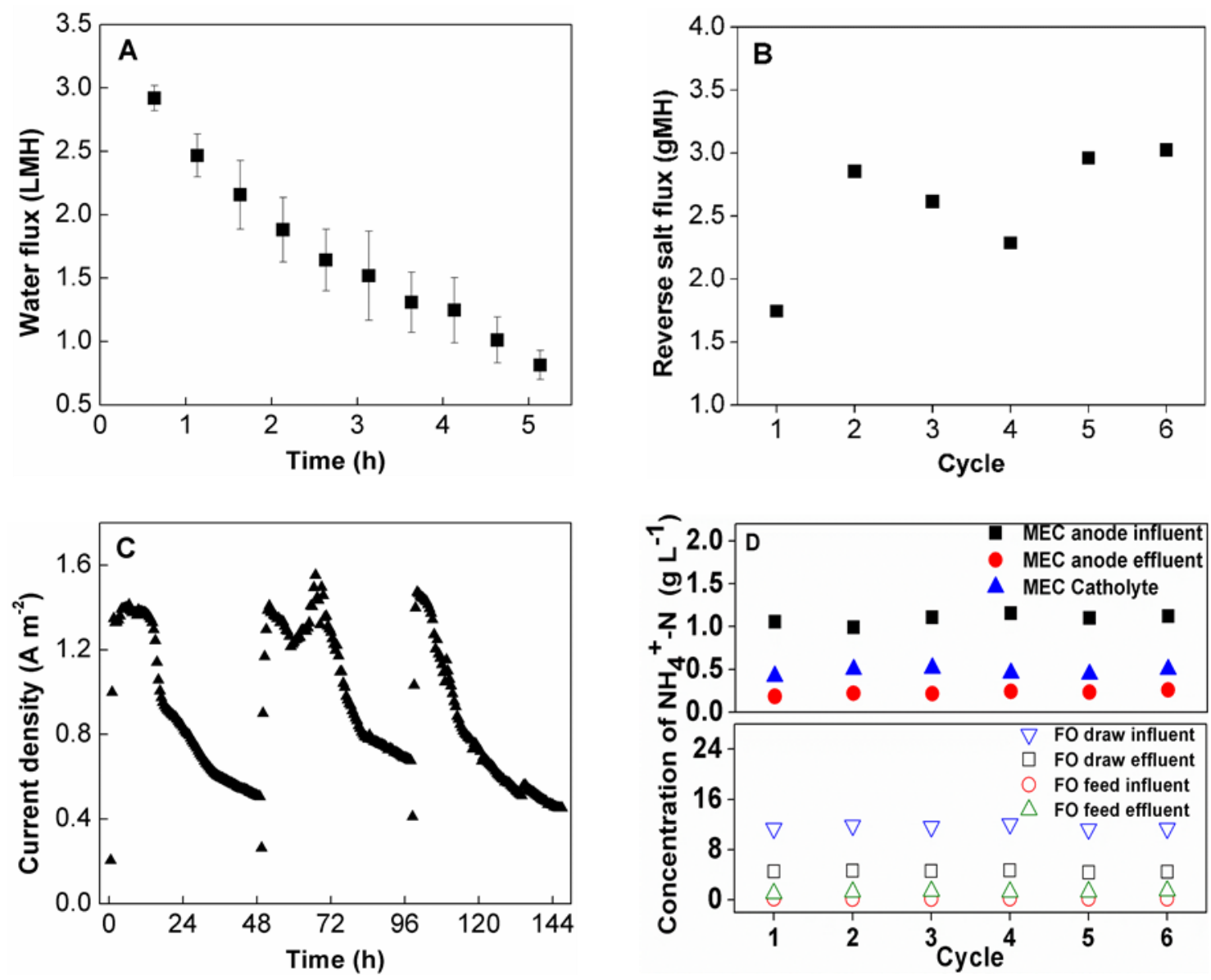

Figure 8.3 The MEC-FO system: (A) water flux in the FO process, (B) reverse salt flux in FO, the $(C)$ current density from the mixture of returned feed solution and fresh anolyte in the MEC, and (D) ammonium nitrogen concentration in the MEC and the FO

The concentrated feed solution from the FO was mixed with fresh anolyte $(\sim 1: 1)$ and then the mixture was fed into the MEC anode compartment in the following cycle. The COD concentration in the mixture was $3.07 \pm 0.28 \mathrm{~g} \mathrm{~L}^{-1}$ and the $\mathrm{NH}_{4}{ }^{+}-\mathrm{N}$ concentration was $1.08 \pm 0.06 \mathrm{~g} \mathrm{~L}^{-1}$. The MEC fed with the mixed influent generated $1.5 \pm 0.1 \mathrm{~A} \mathrm{~m}^{-2}$ (Figure 8.3C), lower than that with fresh feeding solution $\left(1.8 \pm 0.1 \mathrm{~A} \mathrm{~m}^{-2}\right)$, possibly because of lower COD input and accumulation of soluble microbial products (SMP) (Barker and Stuckey 1999, Rittmann and McCarty 2012). The lower current resulted in relatively lower COD removal efficiency of $47.1 \pm 3.5 \%(0.73 \pm$ $\left.0.12 \mathrm{~kg} \mathrm{~m}^{-3} \mathrm{~d}^{-1}\right)$ but had minor influence on the ammonium recovery $(79.7 \pm 2.0 \%, 0.56 \pm 0.04 \mathrm{~kg}$ 
$\left.\mathrm{m}^{-3} \mathrm{~d}^{-1}\right)$. After 6 cycles the conductivity of the MEC anolyte slightly increased from 6.09 to 7.65 $\mathrm{mS} \mathrm{cm}{ }^{-1}$, because of the concentrated salts during the FO process. Increased conductivity in the anolyte could decrease water flux in the FO, due to decreased salt gradient. It is expected that salts will built up over time to a level that will significantly affect water flux; at that time (which needs to be further verified), the anolyte may be disposed and the MEC will receive 100\% fresh organic solution to start a new cycle.

The total input nitrogen in ammonium in one cycle is 0.014 mol, among which $18.6 \%$ stayed in the anolyte, $11.2 \%$ stayed in the catholyte as ammonium and $71.4 \%$ was stripped out as ammonia. In each batch, $0.011 \mathrm{~mol} \mathrm{~N}$ was removed from the anolyte and among them 0.006 mol was recovered to generate ammonium bicarbonate (in the absorption bottle; the rest 0.004 mol was recovered in the Recovery Bottle). The ammonia recovery in the absorption bottle could be improved by decreasing the $\mathrm{pH}$ of the absorption solution (e.g., using acidic solution instead of DI water). During the FO process, $12.1 \%$ of the draw solute (ammonium) was lost every cycle due to the reverse salt flux and $14.9 \%$ was lost in the heat regeneration process. The loss due to reverse salt flux can hardly be avoided because of FO membrane properties; however, the loss during heat regeneration can be reduced with improved regeneration efficiency (the literature reports up to $100 \%$ regeneration). Assuming 100\% regeneration, the lost ammonium in each cycle (because of reverse salt flux) will be about 0.009 mol, which may be resupplied by ammonium recovery in one batch of the MEC at current recovery efficiency.

The total amount of input $\mathrm{C}$ is $0.04 \mathrm{~mol}(0.025 \mathrm{~mol}$ from glucose and $0.015 \mathrm{~mol}$ from sodium bicarbonate). At the end of a batch, $60.6 \%$ of the glucose was oxidized and inorganic carbon was 
generated. The total amount of inorganic carbon was $0.032 \mathrm{~mol}$. Among them, $0.002 \mathrm{~mol}$ was $\mathrm{CO}_{2}$ gas $(47 \mathrm{~mL})$, demonstrating that only $6.3 \%$ of the inorganic carbon was in gaseous phrase and the majority of them existed in the solution. This was due to the low electrolyte $\mathrm{pH}$ (around 5) in the anode chamber. As a result, $\mathrm{CO}_{2}$ was largely present as carbonate acid in the solution (Wojtowicz 2001). Similar to ammonium, the lost $\mathrm{CO}_{2}(0.009 \mathrm{~mol})$ will need multiple batches to be resupplied. As one can see, there is enough $\mathrm{CO}_{2}$ in the anode but most in the water due to low $\mathrm{pH}$. By elevating the anolyte $\mathrm{pH}$, more $\mathrm{CO}_{2}$ may be released. For practical operation of the proposed system, we think additional $\mathrm{CO}_{2}$ supply from an external source will still be required. This external source already exists in wastewater treatment: anaerobic digesters will provide both $\mathrm{CO}_{2}$ (combustion of $\mathrm{CH} 4$ ) and heat energy (the part of the energy that is usually not recovered or used).

Energy consumption in the MEC-FO system included the external power source in MEC, the aeration in MEC, the recirculation of the solution both in MEC and FO. The data can be expressed in $\mathrm{kWh} \mathrm{m}^{-3}$ treated water, $\mathrm{kWh} \mathrm{kg}^{-1} \mathrm{COD}$ (removed) and $\mathrm{kWh} \mathrm{kg}^{-1} \mathrm{~N}$ (removed). In the MEC, the energy input by the external power supply was calculated as (Logan 2008):

$$
P_{\text {power }}=\int_{t=0}^{t} I E d t
$$

where $\mathrm{I}$ is the current $(\mathrm{A}), \mathrm{R}$ is the external resistance $(1 \Omega)$ and dt is the time increment (s). Power requirement by the aeration was calculated as described in a previous publication (Zhang and He 2013b). Power requirement by the recirculation pump was calculated as previous study (Kim et al. 2010). Since the heat used to regenerate ammonium bicarbonate could be provided by the power plant, the heat was not included in the energy input calculation.

In MEC, the energy input was $3.9 \mathrm{kWh} \mathrm{m}^{-3}$ treated water $\left(2.0 \mathrm{kWh} \mathrm{kg}^{-1} \mathrm{COD}, 5.1 \mathrm{kWh}\right.$ $\mathrm{kg}^{-1} \mathrm{~N}$ ). Among them, $53.8 \%$ was consumed by the external power supply, $16.8 \%$ was due to the 
aeration in the cathode, and $29.4 \%$ was consumed by the anolyte recirculation. In FO process, the energy input was $0.1 \mathrm{kWh} \mathrm{m}^{-3}$ treated water, which was much lower than that for MEC. As shown in Table 8.1, if the MEC-FO was replaced with MFC-FO, the energy input will be negative, which means more energy will be produced than needed for the ammonium recovery.

Table 8.1 Comparison between the MEC system, MFC-FO system and MFC

\begin{tabular}{|c|c|c|c|}
\hline & MEC & MFC & $\begin{array}{c}\text { Conventional } \\
\mathrm{NH}_{3} \text { stripping } \\
\end{array}$ \\
\hline Energy input $\left(\mathrm{kWh} \mathrm{kg}^{-1} \mathrm{~N}\right)^{\mathrm{a}}$ & 5.1 & $\begin{array}{c}\text { 3.03(Kuntke et } \\
\text { al. 2012) }\end{array}$ & $\begin{array}{c}\text { 7.31(Maurer et } \\
\text { al. 2003a) }\end{array}$ \\
\hline Net energy yield $\left(\mathrm{kWh} \mathrm{kg}^{-1} \mathrm{~N}\right)$ & -5.1 & $\begin{array}{c}\text { 2.92(Kuntke et } \\
\text { al. 2012) }\end{array}$ & $\begin{array}{c}-9.03 \text { (Maurer et } \\
\text { al. 2003a) }\end{array}$ \\
\hline Nitrogen recovery $\left(\mathrm{kgN} \mathrm{d}^{-1} \mathrm{~m}^{-2}\right)^{b}$ & 7.59 & $\begin{array}{c}\text { 3.29(Kuntke et } \\
\text { al. 2012) }\end{array}$ & - \\
\hline
\end{tabular}

a The energy input includes aeration, recirculation and external power.

$\mathrm{b}$ The production of clean water is vs. FO membrane surface

We have analyzed the mass distribution for both carbon and ammonium, and found that the system would need multiple batches to resupply the lost ammonium and carbon dioxide due to reverse salt flux and during regeneration. Improving regeneration efficiency will make it possible to resupply ammonium via one-batch operation, but $\mathrm{CO}_{2}$ is still insufficient because of low anolyte $\mathrm{pH}$ that retains most $\mathrm{CO}_{2}$ as carbonate acid. External supply of $\mathrm{CO}_{2}$ will be necessary and there is readily available source during wastewater treatment, e.g., combustion of methane gas produced in anaerobic digester that can also provide heat for regeneration of draw solute. Because of the need for supplementing the lost draw solutes, low-strength wastewater may not be a suitable target for the present system because of its low concentrations of both organics and ammonium. The organic solution used here mimicked some types of animal wastewater, and the wastewaters such 
as digestion effluent of other wastes and leachate containing higher concentrations of organics and/or ammonium will also be of interest for this system.

In the MEC, the energy input was $3.9 \mathrm{kWh} \mathrm{m}^{-3}$ treated water $\left(2.0 \mathrm{kWh} \mathrm{kg}^{-1} \mathrm{COD}\right.$, or $5.1 \mathrm{kWh} \mathrm{kg}^{-}$ ${ }^{1} \mathrm{~N}$ ), among which $53.8 \%$ was consumed by the external power supply, $16.8 \%$ was due to the aeration in the cathode, and $29.4 \%$ was consumed by the anolyte recirculation. In the FO, the energy input was $0.1 \mathrm{kWh} \mathrm{m}^{-3}$. To eliminate the major energy requirement by the external power supply, microbial fuel cells (MFCs) may replace MECs for ammonia recovery. We will investigate the use of MFCs and evaluate the relationship between reduced energy consumption and decreased treatment efficiency.

Those results have successfully demonstrated the feasibility of coupling an ammonia-recovering MEC with FO for treating organic solution and recovering valuable resources (e.g., ammonia and water). Such cooperation provides a sustainable source of ammonium-based draw solute for FO. Establishing a loop operation of wastewater between the MEC anode and the FO feed side could alleviate the effect of reverse ammonium flux on the effluent quality with further treatment/recovery in the MEC. The hydraulic retention time in each unit of the system needs to be better coordinated because of uneven treatment speed between the two. Nevertheless, this proofof-concept system encourages further studies of system optimization with examination of actual wastewaters. 


\title{
Chapter 9 Recovery of Nitrogen and Water from Landfill Leachate by A Microbial Electrolysis Cell-Forward Osmosis System
}

(This section has been published as Qin, M., Molitor, H., Brazil, B., Novak, J. T., \& He, Z.* (2016). Recovery of nitrogen and water from landfill leachate by a microbial electrolysis cellforward osmosis system. Bioresource technology, 200, 485-492.)

\begin{abstract}
A microbial electrolysis cell (MEC) - forward osmosis (FO) system was previously reported for recovering ammonium and water from synthetic solutions, and here it has been advanced with treating landfill leachate. In the MEC, $65.7 \pm 9.1 \%$ of ammonium could be recovered in the presence of cathode aeration. Without aeration, the MEC could remove $54.1 \pm 10.9 \%$ of ammonium from the leachate, but little ammonia was recovered. With $2 \mathrm{M} \mathrm{NH}_{4} \mathrm{HCO}_{3}$ as the draw solution, the FO process achieved 51\% water recovery from the MEC anode effluent in 3.5-h operation, higher than that from the raw leachate. The recovered ammonia was used as a draw solute in the FO for successful water recovery from the treated leachate. Despite the challenges with treating returning solution from the FO, this MEC-FO system has demonstrated the potential for resource recovery from wastes, and provide a new solution for sustainable leachate management.
\end{abstract}




\subsection{Introduction}

The amount of solid waste is increasing globally because of increased population and living standards, and thus proper treatment of solid waste is critically important to societal development (Hoornweg and Bhada-Tata 2012). Among waste management options, landfilling is considered as a simple and cost-effective treatment method and often acts as the last destination for the wastes after appropriate treatment (Renou et al. 2008). Landfill leachate, the liquid waste that drains from the landfill, is produced through decomposition of landfill materials and drainage of precipitation that permeates and percolates through the landfill. Landfill leachate is one of the most complex waste streams to manage because it contains a wide range of organic and inorganic compounds including nutrients, chlorinated organics, inorganic salts and heavy metals (Foo and Hameed 2009). Leachate characteristics depend on many factors such as waste composition, landfill age, precipitation, seasonal weather (Kulikowska and Klimiuk 2008, Puig et al. 2011). The presence of toxic compounds in leachate generates problems in both direct disposal to natural water bodies and infiltration into groundwater. Therefore, there is a demand for environmentally sustainable solutions for landfill leachate treatment.

Leachate treatment technologies such as advanced oxidation, membrane separation, chemical treatment, and biological processes have been practiced (Greenman et al. 2009). Especially, biological treatment is widely and effectively employed to remove organic matter and nitrogen from leachate (Gotvajn et al. 2009, Kurniawan et al. 2006). However, leachate usually has a low carbon/nitrogen ratio, which makes the conventional nitrification and denitrification difficult (Puig et al. 2011). Anaerobic ammonium oxidation (anammox) could provide a more sustainable solution for ammonium removal with a high ammonium concentration (Liang and Liu 2008). 
Young landfill leachate contains relatively large amounts of biodegradable organic matter which could be aerobically oxidized, involving both energy consumption and the production of sludge. Removal of organic matter could avoid the negative effects on the anammox process (Ruscalleda et al. 2008). To achieve improved quality of the final effluent, membrane processes, such as microfiltration (MF), ultrafiltration (UF), reverse osmosis (RO), and forward osmosis (FO), are also applied in landfill leachate treatment (Cath et al. 2006, Renou et al. 2008). The major drawback of the implementation of membrane process is membrane fouling, especially for $\mathrm{RO}$ and FO. Thus, pretreatment of landfill leachate to remove organic matter, and to reduce solution conductivity, is required for the membrane process treatment.

Bioelectrochemical systems (BESs) are also studied for simultaneous landfill leachate treatment and energy generation (Damiano et al. 2014, Gálvez et al. 2009, Pant et al. 2010). In BES, such as microbial fuel cells (MFCs) and microbial electrolysis cells (MECs), the organics are oxidized by microorganisms in the anode, and the generated electrons may be used to produce electricity or other valuable chemicals (Wang and Ren 2013). Because of the high conductivity, high buffering capacity, and high ammonium concentration, landfill leachate is considered as a potential substrate for BES (Mahmoud et al. 2014), especially for MECs, which can remove/recover ammonium during electricity generation. In MECs, a higher current density would greatly enhance ammonia recovery, and thus MECs with an external power exhibit a better performance for ammonium recovery than MFCs (Zhang et al. 2014b). Previous studies of leachate treatment by BES focused on the reduction of chemical oxygen demand (COD) in leachate by various types of MFCs (Ganesh and Jambeck 2013, Greenman et al. 2009). It was demonstrated that MFCs could generate electricity from leachate, but the excessive organic residues may decrease Coulombic efficiency 
(CE), especially in membraneless MFC systems (Wu et al. 2015). It was reported that increasing proportions of butyrate and propionate could decrease power density and CE from 1.9 to $1.0 \mathrm{~W} \mathrm{~m}^{-}$ ${ }^{3}$ and from 34 to $20 \%$, respectively (Teng et al. 2010). Although BES could effective remove organic contaminants in leachate with electricity generation, the study of nutrient removal is very limited. Previous studies showed that $50-70 \%$ of the total nitrogen could be removed from landfill leachate with a dual-cathode MFC (Zhang and He 2013b). There have not been any studies that attempted to recover ammonium from leachate in BES.

To explore the potential of resource recovery from landfill leachate, a previously developed MECForward Osmosis (FO) system was adopted here for treating leachate samples. This MEC-FO system was developed to use MECs to recover ammonium from wastewater and use the recovered ammonium as a draw solute in FO for water recovery (Qin and He 2014). This study moved beyond the previous one with examining the waste (leachate) and the effects of aeration. The specific objectives of this study were to: (1) investigate the ammonium recovery from landfill leachate in an MEC; (2) examine the influence of aeration in the MEC cathode on leachate treatment; and (3) explore the synergistic effect of MEC and FO on resource recovery from leachate.

\subsection{Materials and Methods}

\subsubsection{The MEC-FO system}

The MEC-FO system consisted of two units, an MEC and an FO, as shown in Figure 9.1. The operating procedure: the landfill leachate is fed into the anode chamber of the MEC, and its effluent then flows into the feed side of the FO; the collected ammonia gas from the MEC cathode is used to prepare an ammonium bicarbonate solution with external $\mathrm{CO}_{2}$, which is then used as the draw 
solution in the FO; and after water extraction in the FO, the concentrated feed solution is returned to the MEC for further treatment.

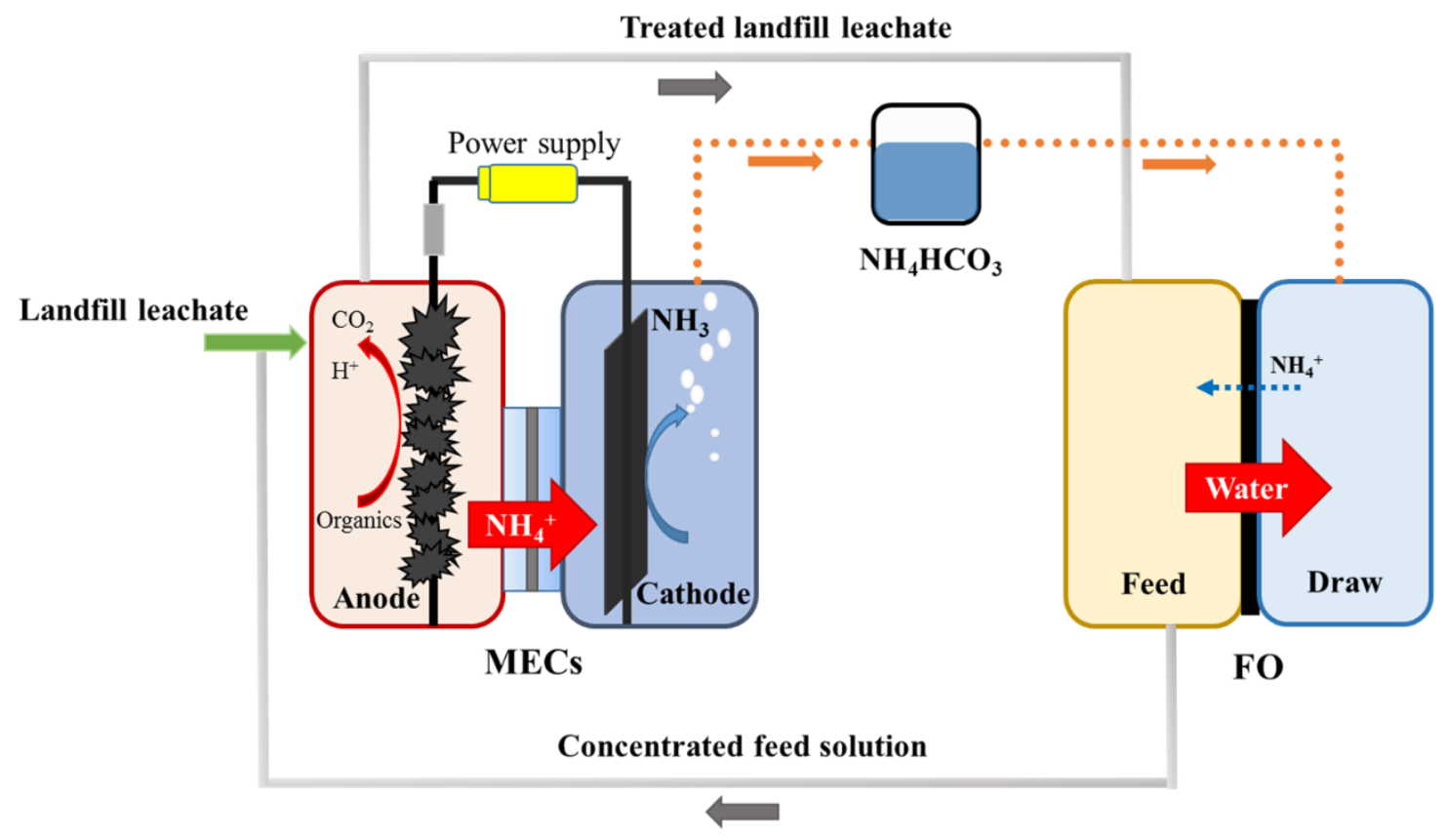

Figure 9.1 Schematic of the MEC-FO system.

\subsubsection{MEC setup and operation}

The MEC had a tubular configuration, with a cation exchange membrane (CEM) tube (Membrane International Inc., Ringwood, NJ, USA) to separate the anode and the cathode. The membrane tube was installed in a glass tube, thereby creating two compartments: the cathode (inside the membrane tube) and the anode (between the membrane and the glass tube). The liquid volume of the anode compartment was $500 \mathrm{~mL}$ and the cathode compartment contained $500 \mathrm{~mL}$ deionized water. A 1m long carbon brush was folded to fit into the anode compartment as the anode electrode. The cathode electrode was a piece of carbon cloth $\left(160 \mathrm{~cm}^{2}\right)$ coated with platinum/carbon as a catalyst (0.3 $\mathrm{mg} \mathrm{Pt} \mathrm{cm}^{-2}$ ) (Zhang et al. 2010). The anode compartment was inoculated with anaerobic sludge collected from the Peppers Ferry Regional Wastewater Treatment Plant (Radford, VA, USA). An 
external voltage of $0.8 \mathrm{~V}$ was applied to the circuit by a power supply (CSI3644A, Circuit Specialists, Inc., Mesa, AZ, USA) according to a previous study (Yossan et al. 2013). The external resistance was fixed at $1 \Omega$ for high current generation. The MEC was operated in a batch mode at room temperature of $\sim 20{ }^{\circ} \mathrm{C}$. Half of the MEC anolyte (landfill leachate) was replaced every 144 $\mathrm{h}$ or $168 \mathrm{~h}$ depending on the hydraulic retention time (HRT) while the catholyte (DI water) remained unchanged. The MEC cathode was operated with either aeration (oxygen reduction) or no aeration (hydrogen evolution). The HRT was 6 days in MEC with aeration and 7 days in MEC without aeration. The difference in HRT in these two modes was due to the different cathode reactions. The HRT was set based on maximum COD removal and current generation. At the beginning of each operation mode, the anolyte and the catholyte were disposed and the MEC received fresh leachate as the anolyte and DI water as the catholyte to start new batch cycles. When aeration was supplied, the air was pumped into the cathode compartment at a rate of $95 \mathrm{~mL} \mathrm{~min}^{-1}$ and the waste air was channeled through two 200-mL glass bottles. The first bottle was an absorption bottle, filled with DI water to collect gaseous ammonia $\left(\mathrm{NH}_{3}\right)$ and carbon dioxide $\left(\mathrm{CO}_{2}\right)$ to generate ammonium bicarbonate draw solute. Additional carbon dioxide was introduced into this bottle at a flow rate of $1.02 \mathrm{~mL} \mathrm{~min}^{-1}$ by a $\mathrm{CO}_{2}$ cylinder. The second bottle was filled with 1 M sulfuric acid $\left(\mathrm{H}_{2} \mathrm{SO}_{4}\right)$ to absorb excess $\mathrm{NH}_{3}$ that was not captured by the first bottle, named "recovery bottle". For the MEC without aeration, a gas bag was used to collect the ammonia gas from the cathode compartment.

\subsubsection{FO setup and operation}

A SEPA CF Cell (Sterlitech Corporation, Kent, WA, USA) was used as an FO cell. The FO membrane was cellulose triacetate (Hydration Technologies Inc., Albany, OR) and was installed 
in the FO cell with its active layer toward the feed solution. During operation, both the feed solution (treated landfill leachate) and the draw solution (ammonium bicarbonate) were recirculated at $125 \mathrm{~mL} \mathrm{~min}^{-1}$. The water flux $\left(\mathrm{L} \mathrm{m}^{-2} \mathrm{~h}^{-1}, \mathrm{LMH}\right)$ was calculated by the change of weight of the feed or draw solution (Wang et al. 2010a). The reverse salt flux $\left(\mathrm{g} \mathrm{m}^{-2} \mathrm{~h}^{-1}, \mathrm{gMH}\right)$ from the draw side to the feed side was calculated by measuring the salt concentration change in the feed solution (Wang et al. 2010a).

\subsubsection{Landfill leachate}

Landfill leachate was collected from a landfill site (Waverly, VA, USA) and kept at $4{ }^{\circ} \mathrm{C}$ prior to use. The leachate was used as collected for all experiments without $\mathrm{pH}$ adjustments, addition of nutrients/trace metals, or dilution. The characterization of the landfill leachate is summarized in Table 9.1. The landfill leachate contained a high COD concentration of $9,175 \mathrm{mg} \mathrm{L}^{-1}$. The average $\mathrm{NH}_{4}{ }^{+}-\mathrm{N}$ concentration was $4,540 \mathrm{mg} \mathrm{L}^{-1}$ while the concentrations of nitrite and nitrate in landfill leachate were below the detection limit of IC $\left(0.125 \mathrm{mg} \mathrm{L}^{-1}\right)$. The $\mathrm{pH}$ of the leachate sample was 7.94, which corresponded to an alkaline environment at the methanogenic phase of decomposition (Greenman et al. 2009). The conductivity was high at $28.1 \mathrm{mS} \mathrm{cm}^{-1}$ due to the high levels of inorganic ions in the samples $\left(\mathrm{Na}^{+}, \mathrm{Cl}^{-}, \mathrm{Mg}^{2+}\right.$ etc.). A variety of heavy metals $(\mathrm{Cu}, \mathrm{Zn}, \mathrm{Ni}, \mathrm{Sr}, \mathrm{Mo}$, Co, etc.) were also detected though at low concentrations. The leachate showed a dark brownreddish color, which may be attributed to the presence of dissolved organic matter (e.g., humic acid and fulvic acid) (Baun and Christensen 2004, Shouliang et al. 2008). 
Table 9.1 Chemical characteristics of the landfill leachate used in this study.

\begin{tabular}{lll}
\hline Parameters & Values & Unit \\
\hline $\mathrm{pH}$ & 7.94 & \\
Conductivity & 28.1 & $\mathrm{mS} \mathrm{cm}^{-1}$ \\
$\mathrm{COD}$ & 9,175 & $\mathrm{mg} \mathrm{L}^{-1}$ \\
$\mathrm{NH}_{4}{ }^{+}-\mathrm{N}$ & 4,540 & $\mathrm{mg} \mathrm{L}^{-1}$ \\
$\mathrm{Na}$ & 3,342 & $\mathrm{mg} \mathrm{L}^{-1}$ \\
$\mathrm{~K}$ & 1,299 & $\mathrm{mg} \mathrm{L}^{-1}$ \\
$\mathrm{Cl}$ & 5,184 & $\mathrm{mg} \mathrm{L}^{-1}$ \\
$\mathrm{Mg}$ & 130.8 & $\mathrm{mg} \mathrm{L}^{-1}$ \\
$\mathrm{Si}$ & 60.9 & $\mathrm{mg} \mathrm{L}^{-1}$ \\
$\mathrm{P}$ & 16.4 & $\mathrm{mg} \mathrm{L}^{-1}$ \\
$\mathrm{Ca}$ & 72.5 & $\mathrm{mg} \mathrm{L}^{-1}$ \\
$\mathrm{Fe}$ & 23.8 & $\mathrm{mg} \mathrm{L}^{-1}$ \\
$\mathrm{Cu}$ & 0.8 & $\mathrm{mg} \mathrm{L}^{-1}$ \\
$\mathrm{Ni}$ & 0.2 & $\mathrm{mg} \mathrm{L}^{-1}$ \\
$\mathrm{Zn}$ & 0.7 & $\mathrm{mg} \mathrm{L}^{-1}$ \\
\hline
\end{tabular}

\subsubsection{Measurement and analysis}

The voltage across the external resistance in the MEC was recorded every 2 min by a digital multimeter (2700, Keithley Instruments Inc., Cleveland, OH, USA). The pH of the liquid stream was measured by a benchtop pH meter (Oakton Instruments, Vernon Hills, IL, USA). Conductivity was measured with a benchtop conductivity meter (Mettler-Toledo, Columbus, OH, USA). The COD concentration was measured using a colorimeter (Hach DR/890, Hach Company, Loveland, CO, USA). The concentration of ammonium was measured using an ISE meter (Accumet AB250, Fisher Scientific, Pittsburgh, PA, USA). Ion concentrations were quantified by using ion chromatography (Dionex LC20 ion chromatograph, Sunnyvale, CA, U.S.A.) equipped with an ED40 eletrochemical detector and inductively coupled plasma - mass spectrometry (Thermo Electron X-Series ICPMS, Waltham, MA, U.S.A.). The concentration of $\mathrm{CO}_{2}$ in the MEC outlet gas was quantified by a Shimadzu GC-14A gas chromatograph equipped with a thermal conductivity meter (TCD). The analysis of electrochemical impedance spectroscopy (EIS) of the FO membrane was conducted with a potentiostat (Reference 600, Gamry Instruments, Warminster, 
PA, USA) according to a previous study (Ge et al. 2013b). The efficiency of organic-to-electricity was expressed by Coulombic recovery (CR) and Coulombic efficiency (CE), calculated as (Ge et al. 2013b):

$$
\begin{gathered}
C R=\frac{Q_{\text {output }}}{Q_{\text {input }-t}}=\frac{\sum I(A) t(s)}{96485\left(\frac{\mathrm{C}}{\text { mol e }^{-}}\right) \times C O D_{\text {total }}(\mathrm{mol}) \times 4\left(\frac{\mathrm{mol}^{-}}{\mathrm{mol} \mathrm{O}_{2}}\right)} \\
C E=\frac{Q_{\text {output }}}{Q_{\text {input }}}=\frac{\sum I(A) t(s)}{96485\left(\frac{C}{\text { mol }^{-}}\right) \times C O D_{\text {removed }}(\mathrm{mol}) \times 4\left(\frac{\text { mol e }^{-}}{\mathrm{mol} \mathrm{o}_{2}}\right)}
\end{gathered}
$$

where $Q_{\text {output }}$ is the produced charge, $Q_{\text {input-t }}$ is the total charge available in the added organic compounds, $Q_{\text {input }}$ is the total charge available in the removed organic compounds, $I$ is electric current and $t$ is the time. $C O D_{\text {total }}$ is the total COD in the anolyte in the period of time $t$, and calculated based on the input COD concentration and the remaining COD from last cycle. $C O D_{\text {removed }}$ is the removed COD within time $t$.

\subsection{Results and Discussion}

\subsubsection{MEC performance}

The MEC performance was evaluated with the leachate sample under two modes, aeration in the cathode (oxygen reduction) and no aeration (hydrogen evolution). In the presence of aeration, oxygen would act as an electron acceptor and the air flow in the cathode compartment could also promote the stripping of ammonia out of the catholyte. In the absence of aeration, protons could be reduced to hydrogen gas. Both reactions can accumulate hydroxyl ions and thus elevate the $\mathrm{pH}$, which would facilitate the conversion of ammonium ion to ammonia gas. 
Electricity generation in the MEC exhibited similar performance regardless of aeration (Figure 9.2A). The maximum current density with aeration was $0.72 \pm 0.06 \mathrm{~A} \mathrm{~m}^{-2}$, similar to $0.70 \pm 0.12$ $\mathrm{A} \mathrm{m}^{-2}$ in the absence of aeration $(p>0.05)$. The MEC with aeration generated the total coulomb of $4,274 \pm 500 \mathrm{C}$ in each batch cycle, which is not significantly different from $3791 \pm 583 \mathrm{C}$ in the absence of aeration $(p>0.05)$. The current generation was relatively stable under both modes but the COD concentration in the MEC effluent increased and thus the COD removal efficiency decreased with time. With aeration, the effluent COD was 4,440 $\mathrm{mg} \mathrm{L}^{-1}$ from the first batch and gradually increased to 5,910 $\mathrm{mg} \mathrm{L}^{-1}$ after 6 batches. Likewise, the effluent COD increased from 4,440 to $8,610 \mathrm{mg} \mathrm{L}^{-1}$ after 5 batches without aeration. This suggests that microbial ability of organic degradation was possibly impaired during operation, likely related to the accumulation of salt ions that will be discussed in the following section. Although the Coulombic efficiency increased from $29.87 \%$ to $46.89 \%$ (aeration) or from $37.61 \%$ to $110.40 \%$ (no aeration), mostly because of the amount of the removed COD decreased, the Coulombic recovery of the MEC was fairly stable at $15.45 \pm 1.80 \%$ (aeration) or $13.70 \pm 2.11 \%$ (no aeration) (Figure 9.2B).

The $\mathrm{pH}$ of the MEC anode effluent was around 7, indicating that the landfill leachate had high buffering capacity. The catholyte $\mathrm{pH}$ remained stable around 10 in both operation modes. The conductivity of both the anolyte and the catholyte increased with operation (Figure 9.2C), because of different reasons: the anolyte conductivity increased probably due to the accumulation of ions, while the catholyte conductivity increased because of the ion transportation from the anolyte across the CEM. 

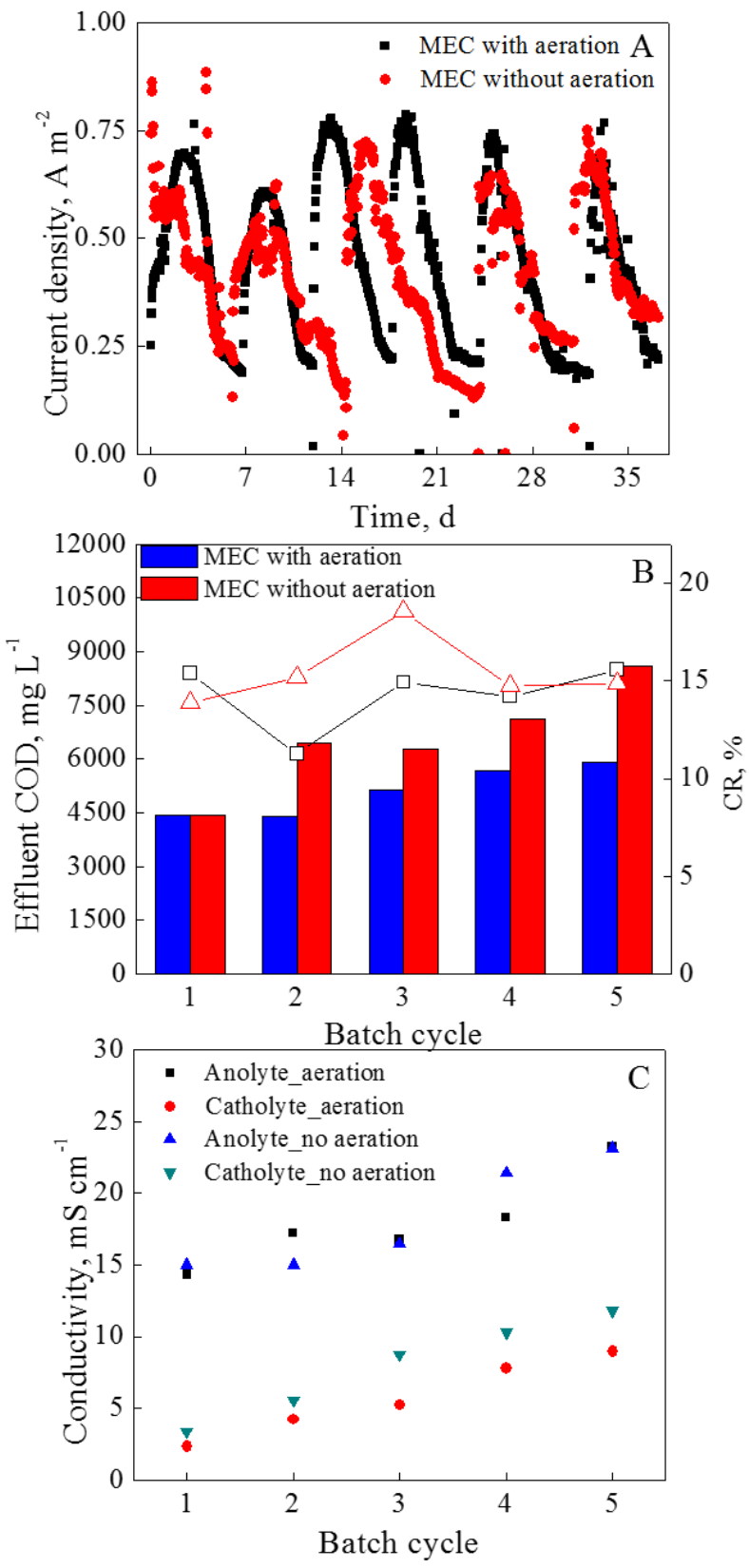

Figure 9.2 The MEC performance fed with and without aeration: (A) current generation; (B) average effluent COD and Coulumbic recovery (CR); and (C) the MEC effluent conductivity.

The MEC effectively decreased the concentration of ammonium in the leachate. When aeration was applied, the anode effluent contained ammonium nitrogen of 1,012 $\pm 109 \mathrm{mg} \mathrm{L}^{-1}$ (Figure 9.3A) representing a removal efficiency of $65.7 \pm 9.1 \%$. Meanwhile, the catholyte had an ammonium concentration of $611 \pm 66 \mathrm{mg} \mathrm{L}^{-1}$, resulting from ammonium migration from the anolyte. The 
stripped ammonia was collected in the absorption bottle, in which the ammonium concentration increased gradually to $0.77 \mathrm{~mol} \mathrm{~L}^{-1}$ after 6 batch cycles. When there was no aeration (the solutions in both anode and cathode compartments were refilled with new solutions before the test), it was observed that the ammonium concentration in both compartments increased with operation batches: the ammonium concentration in the anode effluent increased from $889 \mathrm{mg} \mathrm{L}^{-1}\left(1^{\text {st }}\right.$ batch ) to $1,548 \mathrm{mg} \mathrm{L}^{-1}\left(5^{\text {th }}\right.$ batch) (Figure 9.3B), corresponding to a decrease in ammonium removal efficiency from 68.1 to $47.2 \%$; the ammonium concentration in the catholyte increased from 840 $\mathrm{mg} \mathrm{L}^{-1}\left(1^{\text {st }}\right.$ batch) to $8,202 \mathrm{mg} \mathrm{L}^{-1}\left(5^{\text {th }}\right.$ batch). The inserts in Figure 9.3A and Figure 9.3B indicated that the MEC with aeration achieved higher ammonium removal efficiency and more stable performance than that without aeration. In the absence of aeration, $88.9 \%$ of the input ammonium stayed in the solution (anode effluent and the catholyte) after 5 batch cycles. The anolyte $\mathrm{pH}$ was around 7 , which allowed ammonium nitrogen existing as $\mathrm{NH}_{4}{ }^{+}$. In the catholyte, the $\mathrm{pH}$ was around 10 , and thus $85 \%$ of the ammonium nitrogen was present as ammonia $\left(\mathrm{NH}_{3}\right)($ Koelliker and Kissel 1988). The ammonia gas was dissolved in the catholyte due to its high solubility in aqueous solution. The membrane uptake for ammonium ion could be neglected because membrane sites were mostly occupied by $\mathrm{Na}^{+}$in the CEM. Although $11.1 \%$ of the ammonium nitrogen might have been converted to ammonia gas, the collection of ammonia gas was not successful in the absence of aeration, likely related to the low stripping effect. Even when the catholyte $\mathrm{pH}$ was adjusted to 12 or 12.6 at the beginning of the $4^{\text {th }}$ or the $5^{\text {th }}$ batch, the ammonium concentration in the catholyte was not obviously affected and the catholyte $\mathrm{pH}$ decreased to around 10 rapidly. After the $5^{\text {th }}$ batch, the aeration was put back in the cathode chamber without replacement of either the anolyte or the catholyte: the current generation increased dramatically to $0.89 \mathrm{~A} \mathrm{~m}^{-2}$ and then decreased gradually to $0.31 \mathrm{~A} \mathrm{~m}^{-2}$ in 2 days (Figure 9.3C). With aeration, the cathode reaction changed from hydrogen 
evolution (-0.41 V vs. SHE) to oxygen reduction $(0.82 \mathrm{~V} v s . S H E)$, and the higher reaction potential for the reduction of oxygen increased the cell voltage, as well as the current generation. The aeration helped strip out the ammonia gas from the catholyte and thus facilitated further removal of ammonium from the anode.

In addition to ammonium, the compounds such as metal ions and organics could also migrate from the anolyte into the catholyte across the CEM. The concentrations of several major inorganic ions were monitored in the effluents from the $3^{\text {rd }}$ and the $5^{\text {th }}$ batches (Figure 9.4). Sodium and potassium
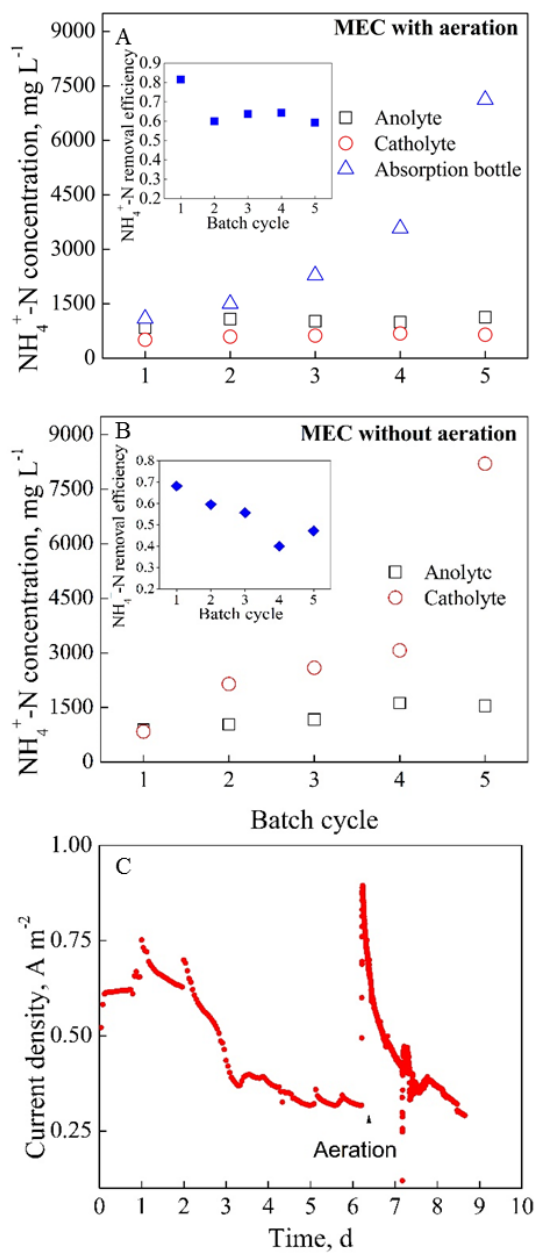

Figure 9.3 Ammonium recovery in the MEC: (A) ammonium concentration with aeration; (B) (A) ammonium concentration without aeration; and (C) the current generation when aeration was put back to the MEC. The insets in (A) and (B) show the $\mathrm{NH}_{4}{ }^{+}-\mathrm{N}$ removal efficiency. 
ions were the dominant cations in both electrolytes while chloride ions were the major anions. The other ions exhibited concentrations lower than $100 \mathrm{mg} \mathrm{L}^{-1}$. In the MEC with aeration, the removal efficiency for $\mathrm{K}^{+}, \mathrm{Na}^{+}$and $\mathrm{Cl}^{-}$was $16.1 \%, 44.7 \%$, and $49.1 \%$ in the $3^{\text {rd }}$ batch, and $9.4 \%, 25.2 \%$, and $40.4 \%$ in the $5^{\text {th }}$ batch, respectively. The lower removal efficiency in $5^{\text {th }}$ batch was likely resulted from the ion accumulation in the catholyte, for example the $\mathrm{Na}^{+}$concentration rose from $449 \mathrm{mg} \mathrm{L}^{-1}$ in the $3^{\text {rd }}$ batch to $950 \mathrm{mg} \mathrm{L}^{-1}$ in the $5^{\text {th }}$ batch, which created a concentration gradient against further migration. The removal efficiency of ions is determined by its concentration and mobility (Ping et al. 2015). The high concentration and high mobility of $\mathrm{K}^{+}, \mathrm{Na}^{+}$and $\mathrm{Cl}^{-}$led to stronger ion migration or diffusion across the membrane, resulting in a higher salinity in the catholyte, which could decrease the catholyte resistance and benefit the current generation. It was observed that more $\mathrm{K}^{+}$and $\mathrm{Na}^{+}$ions were transported across the CEM when there was no aeration, and this was probably related to the lower ammonium transportation under the same condition. Ion transport from the anolyte into the catholyte indicates that MECs may also remove other compounds than ammonium; however, because of nonvolatile feature of those ions, their accumulation in the cathode requires further treatment or periodic replacement of the concentrated solution. 

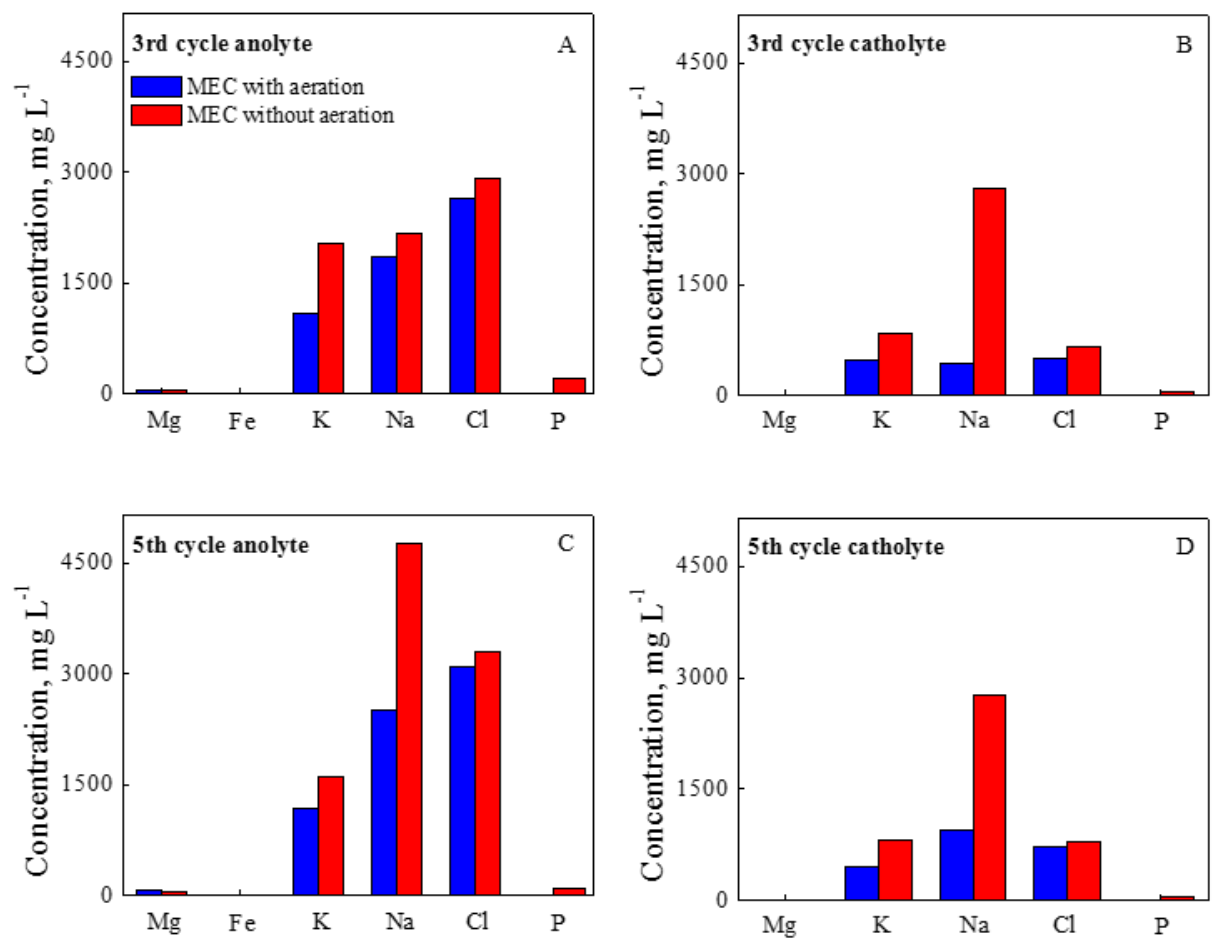

Figure 9.4 The concentrations of the major ions in the MEC with aeration and without aeration (A) in the anolyte from the $3^{\text {rd }}$ batch; (B) in the catholyte from the $3^{\text {rd }}$ batch; $(C)$ in the anolyte from the $5^{\text {th }}$ batch; and (D) in the catholyte from the $5^{\text {th }}$ batch.

After 6 batch cycles, the catholyte exhibited a dark yellow color but continued to be clear. The iron concentration in the catholyte was lower than $0.5 \mathrm{mg} \mathrm{L}^{-1}$ under both operation modes, which was insufficient to cause the observed color. It was found about $22 \%$ of the total organic carbon (TOC) was humic acid, which seems to be a plausible cause of the yellow color (Shouliang et al. 2008). However, after all the humic acid was precipitated out, the yellow color still existed. Therefore, the cause of the yellow color could also be related to other dissolved organic matter such as fulvic acid, and further investigation will be needed to understand the transportation of dissolved organic matter (DOM) across the CEM. 


\subsubsection{FO performance}

Water recovery in the FO was examined with either the raw leachate or the MEC treated leachate, and the prepared ammonium bicarbonate was used as a draw solute. With $1 \mathrm{M}$ ammonium bicarbonate solution as the draw solution, the FO achieved a maximum water flux of $3.3 \pm 0.2$ LMH with the treated leachate (Figure 9.5A), which decreased to $0.6 \pm 0.1 \mathrm{LMH}$ after 3.5 -h operation, and as a result, $26.2 \pm 1.3 \%$ of the treated leachate was extracted into the draw side. The water flux from the raw leachate was much lower, and the maximum water flux was $1.4 \mathrm{LMH}$ (Fig. 5A), resulting in only $12.3 \%$ of the leachate volume extracted into the draw chamber. The greater water flux from the treated leachate benefitted from a lower salt content than the raw (17.4 vs. $28.1 \mathrm{mS} \mathrm{cm}^{-1}$ ), and thus a greater osmotic gradient was created. This indicated that the MEC treatment could improve the FO performance. The FO treatment extracted water and also concentrated the ions in the feed solution (leachate). For example, the ammonium concentration in the feed solution increased from $1,030 \pm 140$ to 7,600 $\pm 300 \mathrm{mg} \mathrm{L}^{-1}$. Reverse flux of ammonium from the draw side into the feed side could also increase the ammonium concentration. When the different concentrations of ammonium bicarbonate were used for draw solution (1 M, $2 \mathrm{M}$ and 4 $\mathrm{M})$, the reverse salt flux was $11.4 \pm 1.4,20.4 \pm 1.4,33.3 \pm 2.5 \mathrm{gMH}$, respectively (Figure 9.5B). The reverse fluxed ammonium could be a concern for further treatment of the treated leachate, and it has been demonstrated that anammox may be used to remove the reverse-fluxed ammonium and thus alleviate the decline of water flux ( $\mathrm{Li}$ et al. 2015). When $2 \mathrm{M} \mathrm{NH}_{4} \mathrm{HCO}_{3}$ was used as draw solution, 51\% water recovery from the MEC effluent was achieved in 3.5-h operation. A higher $\mathrm{NH}_{4} \mathrm{HCO}_{3}$ concentration of $4 \mathrm{M}$ reduced the operating time to $2 \mathrm{~h}$ for achieving $50 \%$ water recovery, and a longer operating time of $3.5 \mathrm{~h}$ could extract $74 \%$ of the water in the leachate sample. 

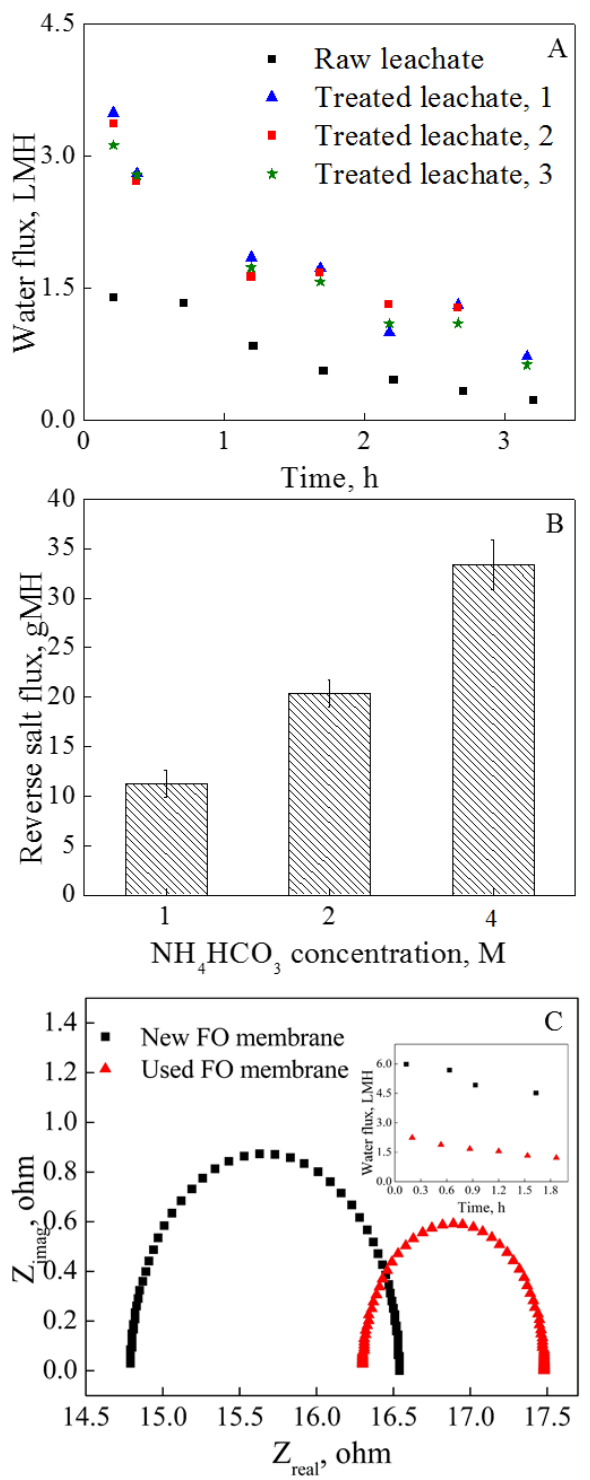

Figure 9.5 The FO performance fed with the treated leachate: (A) water flux with $1 \mathrm{M}$ $\mathrm{NH}_{4} \mathrm{HCO}_{3}$ as draw solution; (B) reverse salt flux with different $\mathrm{NH}_{4} \mathrm{HCO}_{3}$ concentrations; and (C) EIS for new and used FO membranes. The inset in (C) shows the water flux with 0.5 $\mathrm{M} \mathrm{NH}_{4} \mathrm{HCO}_{3}$ as draw solution for new and used $\mathrm{FO}$ membranes.

After 4-month experiment, the FO membrane was examined by measuring the water flux with DI water as the feed solution and $0.5 \mathrm{M} \mathrm{NH}_{4} \mathrm{HCO}_{3}$ as the draw solution. For comparison, a new FO membrane was tested, too. As shown in the insert of Fig. 5C, the maximum water flux of the new 
FO membrane was 6.0 LMH, about 2.7 times higher than that of the used FO membrane $(2.2$ $\mathrm{LMH}$ ), indicating that the membrane fouling decreased the water flux. To further understand the change in the FO membrane, the membrane resistance was measured by using EIS with $30 \mathrm{~g} \mathrm{~L}^{-1}$ $\mathrm{NaCl}$ solution as electrolyte. Figure 9.5C showed the impedance spectra for new and used $\mathrm{FO}$ membranes The measured EIS can be fit by an equivalent circuit according to a previous study (Gao et al. 2013). The first intersection of the Nyquist plot and the $\mathrm{x}$-axis represents the solution + membrane resistance. The new FO membrane had the solution + membrane resistance of 14.79 $\Omega$, slightly lower than $16.30 \Omega$ from the used FO membrane. Because the bulk solution (without FO membrane) had a solution resistance of $13.50 \Omega$, the membrane resistance could be estimated as $1.29 \Omega$ for the new FO membrane or $2.80 \Omega$ for the used FO membrane, and the increase in membrane resistance of the used FO membrane was due to fouling. The charge transfer resistance was $1.75 \Omega$ for new FO membrane and $1.18 \Omega$ for used FO membrane, indicating that ion (e.g., $\mathrm{Na}^{+}$) flux could increase when FO membrane is subjected to fouling (She et al. 2012).

\subsubsection{The MEC-FO system}

When the MEC was linked to the FO unit, the MEC anode effluent (treated leachate) was fed to the FO feed side and the concentrated feed solution was returned to the MEC anode mixed with raw leachate $(\sim 1: 1)$. This mixture had a COD of 11,200 $\pm 1,400 \mathrm{mg} \mathrm{L}^{-1}$. As shown in Figure 9.6A, the maximum current density decreased from $0.76 \mathrm{~A} \mathrm{~m}^{-2}$ to $0.41 \mathrm{~A} \mathrm{~m}^{-2}$ after 3 batch cycles. In addition, the COD removal efficiency decreased from $40.3 \%$ to $32.9 \%$, probably related to the salt accumulation in the anolyte: after 3 batches, the $\mathrm{Na}, \mathrm{Cl}$ and $\mathrm{K}$ concentrations in the MEC anolyte

reached 5,080 $\mathrm{mg} \mathrm{L}^{-1}, 3,067 \mathrm{mg} \mathrm{L}^{-1}$, and 1,846 $\mathrm{mg} \mathrm{L}^{-1}$, respectively. The high salt concentration 
(higher than $100 \mathrm{mM}$ ) could adversely affect the physiology of anaerobic microbial consortia (Lefebvre et al. 2012, Rousseau et al. 2013).
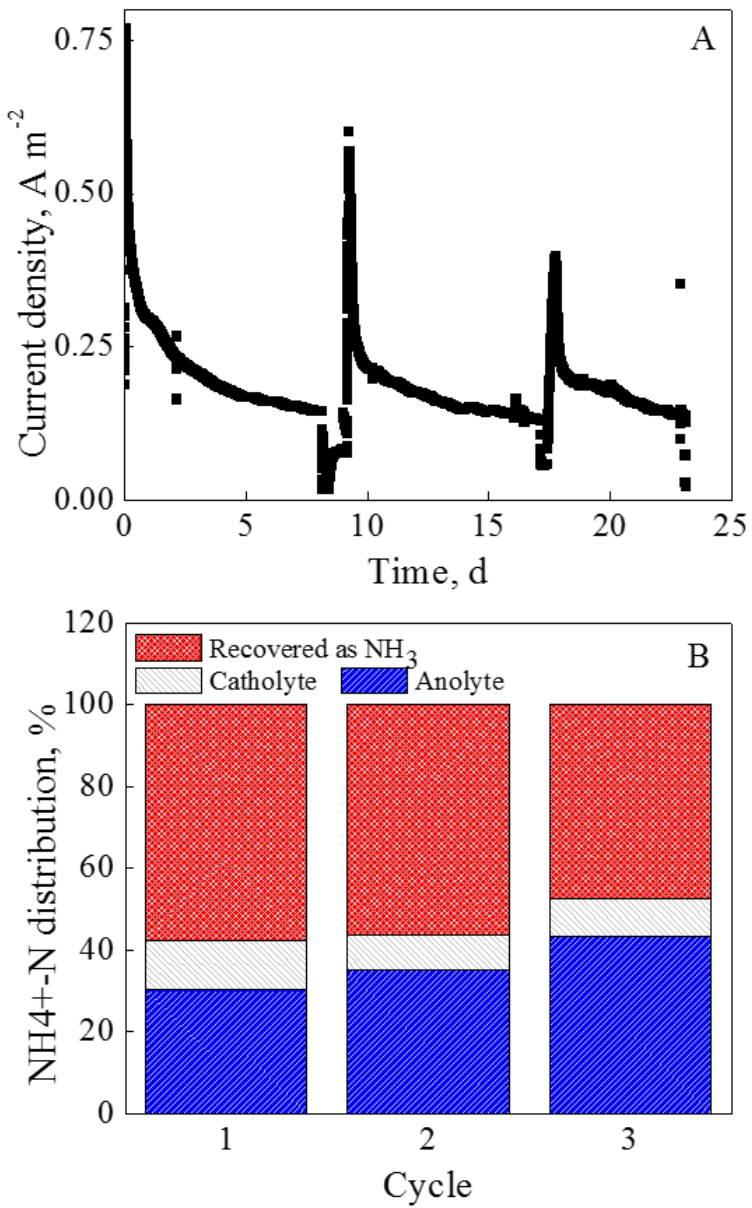

Figure 9.6 The MEC-FO system performance: (A) current generation; and (B) $\mathrm{NH}_{4}{ }^{+}-\mathrm{N}$ distribution during 3 batch cycles.

The MEC removed $63.7 \pm 6.6 \%$ of ammonium in the mixture, and an ammonium mass balance suggested that $36.2 \pm 6.6 \%$ of ammonium stayed in the anolyte, $9.9 \pm 2.0 \%$ was present in the catholyte and $53.8 \pm 5.5 \%$ was stripped out as ammonia gas (Figure 9.6B). The ammonium concentration in anolyte effluent increased from $1,400 \mathrm{mg} \mathrm{L}^{-1}$ to $2,900 \mathrm{mg} \mathrm{L}^{-1}$ during 3 batch cycles, which was due to high ammonium input from the FO returning solution. During the FO process, a large amount of ammonium moved across the FO membrane from the draw side into 
the feed side due to reverse salt flux. The ammonium concentration in the concentrated feed solution was $7,800 \pm 1,600 \mathrm{mg} \mathrm{L}^{-1}$, in which $57.8 \pm 2.3 \%$ was attributed to the reverse salt flux. After the mix with raw leachate, the ammonium concentration decreased to $6,100 \pm 800 \mathrm{mg} \mathrm{L}^{-1}$. The concentration of $\mathrm{Na}^{+}, \mathrm{K}^{+}$and $\mathrm{Cl}^{-}$in the diluted draw solution was 101,49 and $93 \mathrm{mg} \mathrm{L}^{-1}$, respectively, representing salt rejection of $95.8 \%, 94.6 \%$ and $97.8 \%$. No COD was detected in the FO draw effluent.

In the MEC with aeration, the energy input was $5.0 \mathrm{kWh} \mathrm{m}^{-3}$ treated water $\left(1.3 \mathrm{kWh} \mathrm{kg}^{-1} \mathrm{COD}\right.$, or $8.5 \mathrm{kWh} \mathrm{kg}^{-1} \mathrm{~N}$ ), among which $64.4 \%$ was consumed by the external power supply, $13.0 \%$ was due to the aeration in the cathode, and $22.6 \%$ was consumed by the anolyte recirculation. In the MEC without aeration, the energy input was $5.3 \mathrm{kWh} \mathrm{m}^{-3}$ treated water $\left(1.8 \mathrm{kWh} \mathrm{kg}^{-1} \mathrm{COD}\right.$, or 9.7 $\mathrm{kWh} \mathrm{kg}^{-1} \mathrm{~N}$ ). The hydrogen gas was not successfully collected in the MEC without aeration, so the energy consumption was high. In FO process, the energy input was $0.1 \mathrm{kWh} \mathrm{m}^{-3}$ treated water, which was much lower than that for MEC. In the future study, the performance of MFC-FO system will be explored.

The MEC-FO system can be effective for organic removal, nutrient recovery and clean water recovery from leachate. However, there are challenges when the concentrated solution from the FO is returned to the MEC anode. This returned solution increases the concentration of both ions and recalcitrant organic compounds, which make it difficult in the successive treatment. A potential solution for addressing this issue is to introduce pretreatment before the MEC treatment. A proper pretreatment method can improve biodegradability of leachate and thus current generation in the MEC. Higher current output will promote ammonium transport and increase 
ammonium recovery. More ions will also be moved into the cathode of the MEC, thereby alleviating the adverse effects of ion concentrating in the FO and its returning solution. It should be noted that, because ions other than ammonium will be only concentrating in the solution (either the anolyte or the catholyte), periodical replacement of the concentrating solution will be necessary, and the frequency of such replacement will need to be determined experimentally.

\subsection{Conclusions}

This study has furthered the development of an MEC-FO system through examining its performance with actual landfill leachate. The system could effectively generate electricity and recover ammonia. The recovered ammonia was successfully used as a draw solute in the following FO process for water recovery. Aeration in the MEC cathode was found to be necessary for ammonia collection. More water could be covered from the MEC treated leachate than from the raw leachate, because of the reduced salinity. This system has a potential for resource recovery from wastes, and will be investigated for further improvement through pretreatment. 


\title{
Chapter 10 Nanoparticulate $\mathrm{Ni}(\mathrm{OH})_{2}$ films synthesized from macrocyclic nickel (II) Cyclam for hydrogen production in microbial electrolysis cells
}

(This section has been published as Qin, M., Maza, W. A. (co-first author), Stratakes, B. M., Ahrenholtz, S. R., Morris, A. J., \& He, Z. (2016). Nanoparticulate Ni (OH) 2 films synthesized from macrocyclic nickel (II) Cyclam for hydrogen production in microbial electrolysis cells. Journal of the Electrochemical Society, 163(5), F437-F442.)

\begin{abstract}
Hydrogen production in microbial electrolysis cells (MECs) is a promising approach for energy harvesting from wastewater. The kinetic barriers toward proton reduction necessitate the use of catalysts to drive hydrogen formation at appreciable rates and low applied potentials. Towards this end, cost effective alternatives to platinum catalysts are of paramount interest. In this study, $\mathrm{Ni}(\mathrm{OH})_{2}$ films were synthesized by electrophoretic deposition from a Ni(II)cyclam precursor solution at varying concentrations $(6 \mathrm{mM}, 15 \mathrm{mM}$, and $23 \mathrm{mM})$. The films were characterized by scanning electron microscopy and X-ray photo-electron spectroscopy to confirm the deposition of $\mathrm{Ni}(\mathrm{OH})_{2}$. The $\mathrm{Ni}(\mathrm{OH})_{2}$-modified electrodes were then examined by both traditional electrochemical measurements and in an MEC for hydrogen production. Tafel analysis indicates an exchange current density of $\sim 0.36 \mathrm{~mA} \mathrm{~cm}^{-2}$ with a Tafel slope of $\sim 120 \mathrm{mV} \mathrm{decade}{ }^{-1}$ consistent with a rate determining proton adsoprtion step. The hydrogen production rates increased with increasing $\mathrm{Ni}(\mathrm{II})$ cyclam concentration in the precursor solution, with the $23 \mathrm{mM}$-derived film exhibiting a rate comparable to that of a Pt-based catalyst in MEC tests.
\end{abstract}




\subsection{Introduction}

Hydrogen is considered to be one of the most promising energy carriers as an alternative fuel because of its high energy density and availability from renewable sources. Over $90 \%$ of hydrogen gas is produced by steam reforming and coal gasification, both of which are highly energyconsuming processes (Manish and Banerjee 2008). Among the newly developed technologies for hydrogen production, microbial electrolysis cells (MECs) are of special interest as a new approach for hydrogen production from organic matter in wastewater and other organic waste (Logan et al. 2008a, Rozendal et al. 2006b). In an MEC, organic compounds are degraded by exoelectrogens (electrochemically-active microorganisms), and as a result, electrons are passed to an anode electrode. Hydrogen gas can be formed at the cathode (-0.414 V vs. SHE, standard hydrogen electrode) by reducing protons. This process must be aided by an additional voltage ( $0.114 \mathrm{~V}$ in theory) as a result of the insufficiency of the anode potential (e.g., $-0.300 \mathrm{~V}$ vs. SHE when acetate is used as an anode substrate) to drive proton reduction. In reality, an external voltage of more than $0.2 \mathrm{~V}$ is required to overcome the additional kinetic barriers imposed by the hydrogen formation reaction (Kundu et al. 2013). This additional voltage is substantially lower than that needed for electrochemical water splitting (theoretically $1.23 \mathrm{~V}$ vs. SHE (Liu et al. 2005)) making hydrogen production energetically more cost efficient. In addition, recovery of valuable energy content from wastewater/waste also results in environmental benefits.

Catalysts are indispensable in accomplishing the hydrogen evolution reaction (HER). Most MEC studies have used platinum-based catalysts for HER catalysis. Platinum is a highly efficient catalyst for HER, but its high cost obstructs its application in MECs, especially for large scale systems that are designed for wastewater treatment (Kundu et al. 2013, Tartakovsky et al. 2009). 
Thus, alternative catalysts have been explored such as Pd- (Huang et al. 2011), Fe- (Xiao et al. 2012), Mo-(Yuan et al. 2014), and Ni-based materials (Manuel et al. 2010). Among them, Nicontaining nanoparticles are of special interest because of nickel's low cost, abundance, low overpotentials toward proton reduction, and high stability in solutions, which are usually alkaline in the MEC cathode (Kundu et al. 2013, Selembo et al. 2009). For example, a cathode catalyst was developed by electrodepositing NiMo onto a three-dimensional carbon-fiber-weaved cloth material, and at an applied voltage of $0.6 \mathrm{~V}$, the MECs achieved a hydrogen production rate of $0.09 \mathrm{~m}^{3} \mathrm{~m}^{-2} \mathrm{~d}^{-1}\left(\mathrm{~m}^{3} \mathrm{H}_{2}\right.$ per $\mathrm{m}^{2}$ cathode surface area per day) at a sustained current density of $12 \mathrm{~A}$ $\mathrm{m}^{-2}$ (A per $\mathrm{m}^{2}$ cathode surface area) (Hu et al. 2009). The rate of hydrogen production by the NiMomodified carbon cloth was comparable to that of a Pt-modified carbon cloth $\left(0.10 \mathrm{~m}^{3} \mathrm{~m}^{-2} \mathrm{~d}^{-1}\right)$. Commercially available nickel was also used as a catalyst coated on carbon cloth for MEC cathodes (Selembo et al. 2010). Nickel oxide catalysts have also shown great promise as catalysts for hydrogen evolution in MECs (Jeremiasse et al. 2010). Electrodeposition of nickel oxide onto stainless steel and nickel alloy surfaces have been shown to improve the MEC hydrogen production from $0.0002 \mathrm{~m}^{3} \mathrm{~m}^{-2} \mathrm{~d}^{-1}$ (no Ni oxide) to $0.019 \mathrm{~m}^{3} \mathrm{~m}^{-2} \mathrm{~d}^{-1}$ at a current density of $3.25 \mathrm{~A}$ $\mathrm{m}^{-2}$ when a $0.9 \mathrm{~V}$ external voltage bias was applied (Selembo et al. 2009).

In this study, a nano- $\mathrm{Ni}(\mathrm{OH})_{2}$ modified cathode was developed by electrodeposition from a nickel(II) cyclam (cyclam = 1, 4, 8, 11-tetraazacyclotetradecane) precursor. The synthetic method was based on a procedure of the deposition of $\mathrm{Ni}(\mathrm{OH})_{2}$ using $\left[\mathrm{Ni}(\mathrm{en}=\text { ethylene diamine })_{3}\right] \mathrm{Cl}_{2}$, resulting in homogeneous, well adhered $\mathrm{Ni}(\mathrm{OH})_{2}$ thin film (Selembo et al. 2010, Singh et al. 2013). The $\left[\mathrm{Ni}(\mathrm{en})_{3}\right] \mathrm{Cl}_{2}$-derived $\mathrm{Ni}(\mathrm{OH})_{2}$ films were shown to exhibit significantly higher catalytic activity towards water oxidation than films derived from $\left[\mathrm{Ni}\left(\mathrm{OH}_{2}\right)_{6}\right]^{2+}$ solutions under similar 
conditions (Singh et al. 2013). The morphology and composition of the films generated here were investigated via scanning electron microscope (SEM) and X-ray photoelectron spectroscopy (XPS). The catalytic activity toward hydrogen production was examined through electrochemical techniques and in an MEC.

\subsection{Materials and Methods}

Materials — Reagents or analytical grade chemicals were sourced from commercial suppliers and used as received unless stated otherwise. Deionized water was used throughout the study.

Synthesis procedure - Ni(II)cyclam was prepared by refluxing equimolar Nickel(II) chloride hexahydrate (99.3\%, Alfa Aesar) and 1,4,8,11-tetraazacyclotetradecane (> $95 \%$, Ark Pharm Inc) in DMF (99.8 \% spectrophotochemical grade, Spectrum) at $90{ }^{\circ} \mathrm{C}$ for 12 hours. The purple precipitate was filtered and washed with a copious amount of DMF to remove any excess $\mathrm{Ni}(\mathrm{II}) \mathrm{Cl}_{2}$ $6 \mathrm{H}_{2} \mathrm{O}$ and then allowed to dry. This method differs slightly from typical synthetic approaches in which equimolar amounts of $\mathrm{Ni}(\mathrm{II}) \mathrm{Cl}_{2}$ and the tetraazacyclotetradecane are dissolved in methanol or ethanol and the product separated by addition of ether (Bosnich et al. 1965, Voelcker et al. 2008). Our approach affords a precipitated product in nearly quantitative yields.

The $\mathrm{Ni}(\mathrm{OH})_{\mathrm{x}}$ films were electrodeposited onto carbon cloth (PANEX 30PW03, Zoltek Corporation, St. Louis, MO, USA) with an area of $4 \mathrm{~cm}^{2}(2 \mathrm{~cm} \times 2 \mathrm{~cm})$ by dissolving the $\mathrm{Ni}(\mathrm{II})$ cylcam precursor in a solution of $0.1 \mathrm{M} \mathrm{NaOH}$. The potential was then cycled between $0 \mathrm{~V}$ and $1.3 \mathrm{~V}$ (vs $\mathrm{Ag} / \mathrm{AgCl}$ ) for 150 cycles. A three electrode arrangement was employed using the carbon cloth as the working electrode, a platinum mesh counter electrode, and $\mathrm{Ag} / \mathrm{AgCl}$ ( $\mathrm{sat} . \mathrm{KCl}$ ) 
as the reference electrode. The electrodeposition was carried out in a one-compartment electrochemical cell under aerobic conditions at room temperature. The procedure employed was similar to that used by Singh, et. al. for the deposition of $\mathrm{Ni}(\mathrm{OH}) \mathrm{x}$ films onto glassy carbon electrodes and fluorine doped tin oxide electrodes from $\mathrm{Ni}\left(\mathrm{NH}_{3}\right)_{6}, \mathrm{Ni}\left(\mathrm{OH}_{2}\right)_{6}, \mathrm{Ni}(\mathrm{en})_{3}(\mathrm{en}=1,2-$ diaminoethane), and other $\mathrm{Ni}(\mathrm{II})$ amine molecular precursors (Singh et al. 2013). The nomenclature of nano-scaled $\mathrm{Ni}(\mathrm{OH})_{2}$ represents the nickel hydroxide prepared with $\mathrm{X} \mathrm{mM} \mathrm{Ni(II)}$ cyclam as the precursor where X could be 6, 15, and 23.

Characterization - LEO (Zeiss) 1550 field emission scanning electron microscope (FESEM) operating at an acceleration voltage of $300 \mathrm{KV}$ was employed to study the morphology and structural properties of the nanoparticulate films. FEI Quanta 600 FEG environmental scanning electron microscope (ESEM) with Bruker energy-dispersive X-ray spectroscopy (EDS) was used to analyze the elemental composition of the catalyst. XPS was conducted using a PHI 5300 spectrometer with a Perkin-Elmer Dual Anode X-ray source operating with monochromatic Mg $\mathrm{K} \alpha$ radiation (hv $1253.6 \mathrm{eV}$ ) at $13 \mathrm{kV}$ and $250 \mathrm{~W}$ and a pass energy of $17.9 \mathrm{eV}$. A step size of 0.1 eV was used and 256 sweeps were averaged for all measurements. The photoelectrons emitted were detected by a hemispherical analyzer. Operating pressure in the sampling chamber was below 1 x $10^{-7}$ Torr. The spectral range for Ni $2 p$ was $894-844 \mathrm{eV}, \mathrm{N} 1 \mathrm{~s}$ was $410-390 \mathrm{eV}$, and O $1 \mathrm{~s} 545-$ $525 \mathrm{eV}$. The spectra were calibrated according to the $\mathrm{C} 1 \mathrm{~s}$ peak at $284.6 \mathrm{eV}$.

Electrochemical analysis - The synthesized catalysts were coated onto a piece of rectangular carbon cloth (PANEX 30PW03, Zoltek Corporation, St.Louis, MO, USA) with an area of $4 \mathrm{~cm}^{2}$ $(2 \mathrm{~cm} \times 2 \mathrm{~cm})$, which was used as a cathode electrode. A cathode coated with $0.5 \mathrm{mg} \mathrm{cm}^{-2} \mathrm{Pt} / \mathrm{C}$ 
(10\% wt. Platinum on Carbon Vulcan, Fuel Cell Earth LLC, Wakefield, Ma, USA) was prepared and served as a control.

Tafel plots (scan rate $1 \mathrm{mV} \mathrm{s}^{-1}$ ) were prepared with chronoamperametric data obtained on a potentiostat (Reference600, Gamry Instruments, Warminster, PA, USA). Electrochemical experiments were conducted using a three-electrode arrangement in a PBS (1M, $120 \mathrm{~mL})$ electrolyte solution. The modified cathode electrodes served as a working electrode, a platinum wire served as the counter electrode, and an $\mathrm{Ag} / \mathrm{AgCl}$ electrode (CH Instruments, Inc., Austin, TX, USA) was used as a reference electrode. The electrochemical cell was sparged with nitrogen gas for 15 min prior to each test.

MEC setup and operation - A two-chamber MEC (Figure 10.1) was built by connecting two glass bottles with a cation exchange membrane (CEM) as separator (UltexCMI7000, Membranes International, Inc., GlenRock, NJ, USA), according to a previous study (Yuan et al. 2014). The liquid volume of the anodic chamber and the cathodic chamber was 130 and $140 \mathrm{~mL}$, respectively. The anode electrode was a carbon brush (Gordon Brush Mfg.Co., Inc., Commerce, CA, USA) which had been cultivated under a microbial fuel cell mode for three months. The anolyte contained (per liter of DI water): sodium acetate $(1 \mathrm{~g}), \mathrm{NaCl}(1 \mathrm{~g}), \mathrm{MgSO}_{4}(0.015 \mathrm{~g}), \mathrm{CaCl}_{2}(0.02$ $\mathrm{g}), \mathrm{KH}_{2} \mathrm{PO}_{4}(0.53 \mathrm{~g}), \mathrm{K}_{2} \mathrm{HPO}_{4}(1.07 \mathrm{~g}), \mathrm{NaHCO}_{3}(1 \mathrm{~g})$, and trace element $(1 \mathrm{~mL})$ (Angenent and Sung 2001). PBS (1 M, $\left.\mathrm{KH}_{2} \mathrm{PO}_{4}(53 \mathrm{~g} / \mathrm{L}), \mathrm{K}_{2} \mathrm{HPO}_{4}(107 \mathrm{~g} / \mathrm{L})\right)$ solution was used as a catholyte. An external voltage of $0.8 \mathrm{~V}$ was applied to the circuit by a power supply (CSI3644A, Circuit Specialists, Inc., Mesa, AZ, USA) according to a previous study (Yossan et al. 2013). The MEC 
was operated under a batch mode with an HRT of $30 \mathrm{~h}$. In each cycle, part of the anolyte $(60 \mathrm{~mL})$ and the whole catholyte $(140 \mathrm{~mL})$ were replaced.

The voltage across resistor was recorded with a digital multimeter (2700, Keithley Instruments, Inc., Cleveland, OH, USA) with a time interval of 5 min. Current was calculated by Ohm's law. Hydrogen production in the MEC was collected by water displacement and measured with a syringe. Chemical oxygen demand (COD) was measured with a DR/890 colorimeter (HACH Co., Ltd., USA) according to the manufacturer's instructions. The content of $\mathrm{H}_{2}$ was analyzed using a Shimadzu GC-14A gas chromatograph equipped with a thermal conductivity detector (TCD).

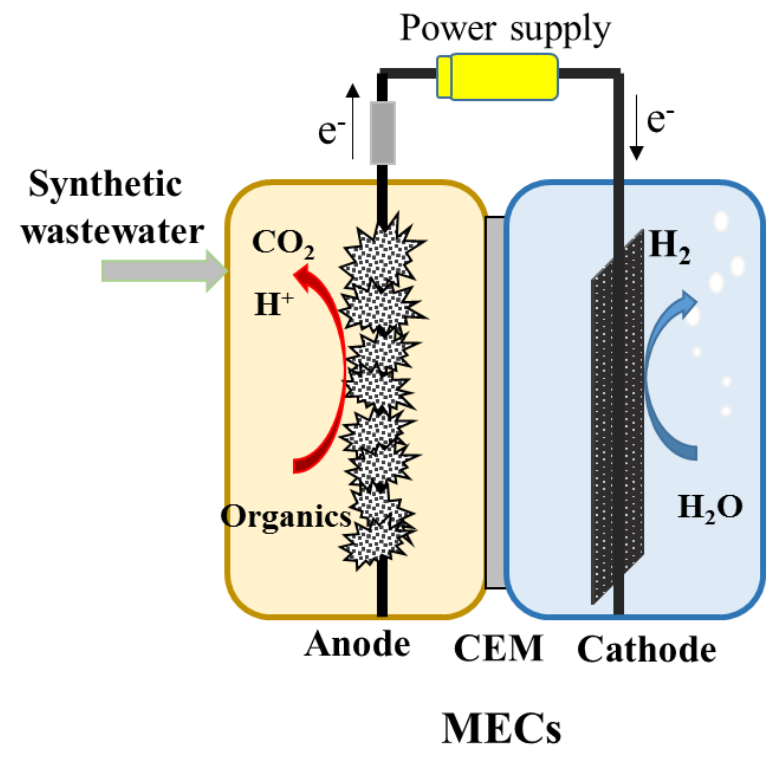

Figure 10.1 Schematic of an MEC

Calculation - Coulombic efficiency, cathodic hydrogen recovery, overall hydrogen recovery and hydrogen production rates were used to evaluate reactor performance (Logan et al. 2008a, Xiao et al. 2012). 
The total charge $\left(C_{\text {total }}, C\right)$ generated by each batch cycle can be calculated based on the measured current:

$$
C_{\text {total }}=\int I d t
$$

where $I$ is the current (A) calculated from the voltage across the resistor $(10 \Omega)$ and $d t$ is the time interval (300 s) for data collection.

The Coulombic recovery $\left(C_{R}\right)$ is the ratio of electrons recovered from substrate consumption relative to the total possible electrons available due to substrate consumption, calculated as

$$
C_{R}=\frac{C_{\text {total }}}{n F \triangle C O D}
$$

where $n$ is the number of electrons released from each oxygen (4), $F$ is the Faraday constant $\left(96,485 \mathrm{C} \mathrm{mol}^{-1} \mathrm{e}^{-}\right)$, and $\triangle C O D$ is total mole of consumed bacterial feedstock within time $t$.

The cathodic hydrogen recovery $\left(R_{c a t}\right)$ is the fraction of electrons that are recovered as hydrogen gas from the total generated electrons:

$$
R_{\text {cat }}=\frac{2 n_{\mathrm{H}_{2}} F}{C_{\text {total }}}
$$

where $n_{H 2}$ is the actual number of hydrogen moles produced.

The overall hydrogen recovery $\left(R_{H_{2}}\right)$ is the ratio of generated hydrogen gas compared to the theoretical hydrogen generation based on substrate degradation:

$$
R_{H_{2}}=C_{R} * R_{c a t}
$$


The hydrogen production rate $\left(Q_{H 2}, \mathrm{~m}^{3} \mathrm{H}_{2} \mathrm{~m}^{-2} \mathrm{~d}^{-1}\right)$ is calculated based on the generated hydrogen gas $\left(\mathrm{m}^{3}\right)$ per $\mathrm{m}^{2}$ cathode electrode per day.

\subsection{Results and Discussion}

$\mathrm{Ni}(\mathrm{II})$ catalyst electrodeposition and electrode preparation. - Cyclic voltammograms (CVs) of a carbon felt electrode (CFE) in the presence of $23 \mathrm{mM} \mathrm{Ni(II)cyclam} \mathrm{in} \mathrm{a} \mathrm{solution} \mathrm{of} 0.1 \mathrm{M} \mathrm{NaOH}$ are shown in Figure 10.2a. The CVs display an anodic peak at $410 \mathrm{mV}$ (vs. Ag/AgCl) whose current density increases with increasing potential sweeps. The increase in current density is accompanied by a shift in the anodic peak to more negative potential up to $285 \mathrm{mV}$. The observed current increase with respect to sweep number is attributed to the deposition of nanoparticulate $\mathrm{Ni}(\mathrm{OH})_{2}$ species. This is consistent with literature reports of $\mathrm{Ni}(\mathrm{OH})_{2}$ deposition from soluble molecular $\mathrm{Ni}(\mathrm{II})$ coordination complexes as pre-catalysts on glassy carbon (GCE) as well as fluorine-doped tin oxide (FTO) electrodes in basic borate buffers and $\mathrm{NaOH}$ solutions (Singh et al. 2013). The $\mathrm{Ni}(\mathrm{OH})_{2}$ has been proposed to form by a $\mathrm{Ni}-\mathrm{N}$ de-ligation mechanism in the presence of strongly coordinating basic groups (Singh et al. 2013).

A plot of the anodic peak current as a function of the sweep number displays a monotonic increase in the concentration current density with a plateau at approximately $420 \mathrm{~mA} \mathrm{~cm}^{-2} \mathrm{M}^{-1}$. Varying the starting solution concentration of $\mathrm{Ni}(\mathrm{II})$ cyclam had little effect on the behavior of the concentration-normalized current densities and value of the observed plateau, between $400 \mathrm{~mA}$ $\mathrm{cm}^{-2} \mathrm{M}^{-1}$ and $470 \mathrm{~mA} \mathrm{~cm}^{-2} \mathrm{M}^{-1}$ (Figure 10.3). The steepness of the inflections differ significantly 
in going from $6 \mathrm{mM} \mathrm{Ni(II)cyclam} \mathrm{to} \mathrm{higher} \mathrm{concentrations} \mathrm{of} \mathrm{Ni}(\mathrm{II})$ cyclam, possibly suggesting a concentration dependent mechanism of film formation.

A

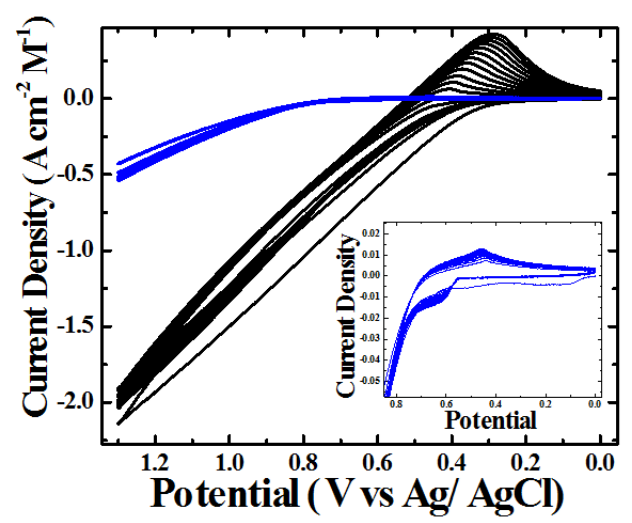

B

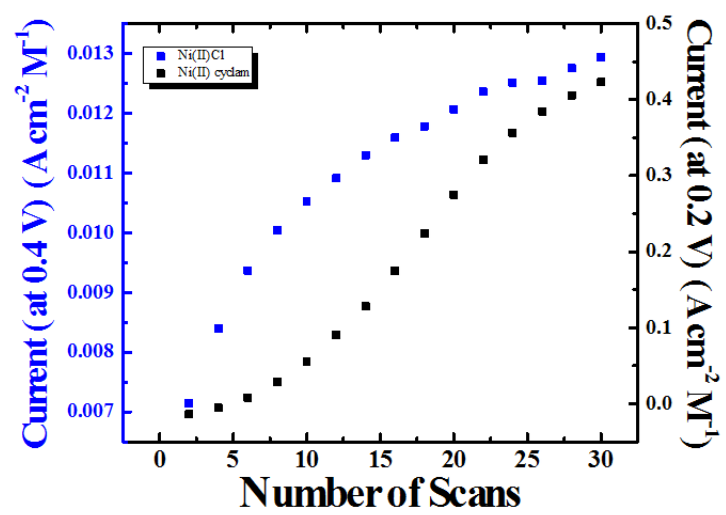

Figure 10.2 (a) Cyclic voltammograms of $\mathrm{Ni}$ (II)cyclam (solid black line) and $\mathrm{Ni}(\mathrm{II}) \mathrm{Cl}_{2}{ }^{\circ} 6 \mathrm{H}_{2} \mathrm{O}$ in an aqueous $0.1 \mathrm{M} \mathrm{NaOH}$ solution. The inset shows a closer view of the $\mathrm{Ni}\left(\mathrm{II}_{1}\right) \mathrm{Cl}_{2} \cdot 6 \mathrm{H}_{2} \mathrm{O}$ CV. (b) Comparison of the current densities corresponding to the electrodeposition of the $\mathrm{Ni}$ (II) catalyst from the $\mathrm{Ni}(\mathrm{II})$ cyclam (black) and $\mathrm{Ni}\left(\mathrm{III} \mathrm{Cl}_{2}\right.$ solutions on the $\mathrm{CFE}$ as a function of the number of potential sweep scans.

For comparison, the electrodeposition of $\mathrm{Ni}(\mathrm{OH})_{2}$ films onto $\mathrm{CFE}$ from a $\mathrm{Ni}(\mathrm{II}) \mathrm{Cl}_{2} \cdot 6 \mathrm{H}_{2} \mathrm{O}$ solution was also carried out (Figure 10.2). The $\mathrm{CV}$ of $\mathrm{Ni}(\mathrm{II}) \mathrm{Cl}_{2} \cdot 6 \mathrm{H}_{2} \mathrm{O}$ in $0.1 \mathrm{M} \mathrm{NaOH}$ displays cathodic and anodic peaks at $520 \mathrm{mV}$ and $440 \mathrm{mV}$, respectively. Upon continued sweeps the anodic peak potential was observed to shift to slightly more positive potentials reaching a value of $460 \mathrm{mV}$ after 30 sweeps. Much like the electrodeposition from Ni(II)cyclam, nickel oxide formation from $\mathrm{Ni}(\mathrm{II}) \mathrm{Cl}_{2}$ on the surface of the CFE (as evidenced by increasing the anodic peak current density) 
is monotonic as a function of increasing number of potential sweeps. Due to the poor solubility of $\mathrm{Ni}(\mathrm{II}) \mathrm{Cl}_{2} \cdot 6 \mathrm{H}_{2} \mathrm{O}$ in $0.1 \mathrm{M} \mathrm{NaOH}$ the effect of $\mathrm{Ni}(\mathrm{II}) \mathrm{Cl}_{2} \cdot 6 \mathrm{H}_{2} \mathrm{O}$ concentration on $\mathrm{Ni}(\mathrm{OH})_{2}$ formation and deposition could not be probed.
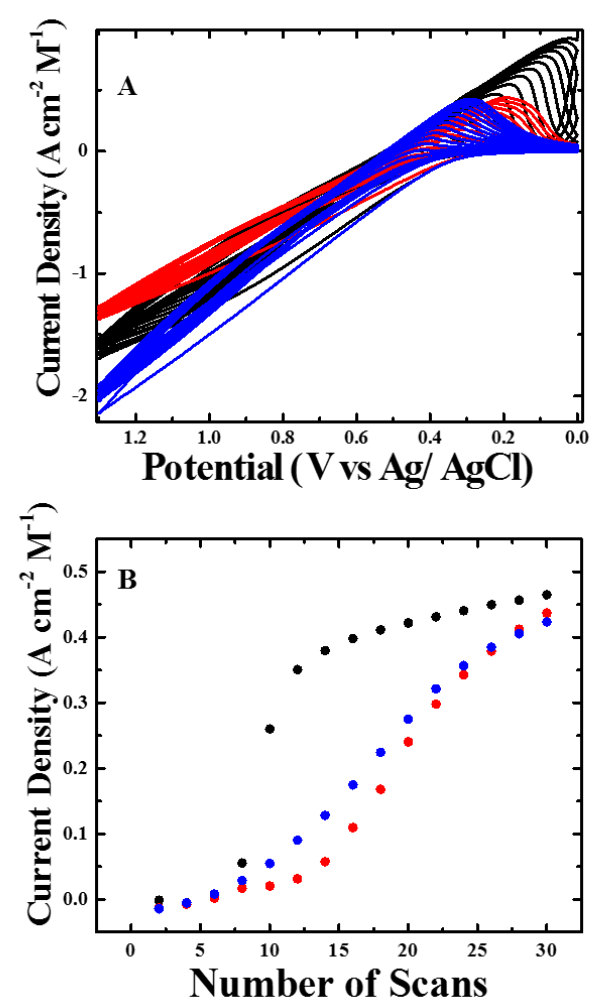

Figure 10.3 (a) Cyclic voltammograms of $6 \mathrm{mM}$ (solid black line), $15 \mathrm{mM}$ (solid red line), and $23 \mathrm{mM}$ (solid blue line) $\mathrm{Ni}$ (II)cyclam solutions in aqueous $0.1 \mathrm{M} \mathrm{NaOH}$ electrolyte. (b) Comparison of the current densities corresponding to the electrodeposition of the $\mathrm{Ni}$ (II) catalyst from the $6 \mathrm{mmm}$ (black circles), $15 \mathrm{mM}$ (red circles), and $23 \mathrm{mM}$ (blue circles) Ni(II)cyclam solutions on the CFE as a function of the number of potential sweep scans. These are compared to each other by dividing the current densities by the concentration of $\mathrm{Ni}$ (II) cyclam at the start of the deposition.

Cyclic voltammetry was performed on the $\mathrm{Ni}(\mathrm{OH})_{2}$ films on CFE and compared to $\mathrm{Ni}(\mathrm{II})$ cyclam in anaerobic $0.1 \mathrm{M} \mathrm{TBAPF}_{6}$ solutions in $\mathrm{CH}_{3} \mathrm{CN}$. At a scan rate of $25 \mathrm{mV} \mathrm{s}^{-1}$ the $\mathrm{CV}$ of the $\mathrm{Ni}(\mathrm{OH})_{2}$ film displays cathodic features at $-0.785 \mathrm{~V}$ and $-1.03 \mathrm{~V}$ with a anodic feature at $-0.555 \mathrm{~V}$ (Figure 10.4). Similar cathodic features at $-0.95 \mathrm{~V}$ and $-1.22 \mathrm{~V}$ with an cathodic feature at $-0.68 \mathrm{~V}$ were 
observed in the $\mathrm{CV}$ of $\mathrm{Ni}(\mathrm{II})$ cyclam at $25 \mathrm{mV} \mathrm{s}^{-1}$. The origin of these features are, as of yet, uncertain. They are, however, consistent with results observed for $\mathrm{Ni}(\mathrm{OH})_{2}$ films deposited from other $\mathrm{Ni}(\mathrm{II})$-amine coordination complexes (Singh et al. 2013). Their presence in the $\mathrm{Ni}(\mathrm{OH})_{2}$ films lead us to believe that they may be related to redox activity of $\mathrm{Ni}(\mathrm{OH})_{2}$ adsorbed on the electrode surface that may have formed during polarization (Ding et al. 1997). It is important to note that the reversible redox features observed with $\mathrm{Ni}(\mathrm{II})$ cyclam with $\mathrm{E}_{1 / 2}=0.398 \mathrm{~V}$ and -1.61 $\mathrm{V}$ (vs $\mathrm{Ag} / \mathrm{AgCl}$ ) corresponding to the $\mathrm{Ni}^{3+2+}$ and $\mathrm{Ni}^{2+/+}$ redox couples, respectively, are not observed in the $\mathrm{Ni}(\mathrm{OH})_{2}$ film (Beley et al. 1986, Jarzębińska et al. 1999, Zhalko-Titarenko et al. 1990, Zhang et al. 2014c).

Characterization of the nickel oxide films. - X-ray photoelectron spectroscopy was performed for the films deposited on CFE from $6 \mathrm{mM}, 15 \mathrm{mM}$, and $23 \mathrm{mM} \mathrm{Ni(II)cyclam} \mathrm{solutions} \mathrm{in} \mathrm{an}$ attempt to elucidate the nature of the films. The spectra corresponding to the $\mathrm{Ni} 2 \mathrm{p}_{3 / 2}, \mathrm{~N} 1 \mathrm{~s}$, and $\mathrm{O}$ 1s electron binding energies are shown in Figure 10.5. The Ni $2 p_{3 / 2}$ and $\mathrm{O} 1 \mathrm{~s}$ spectra were decomposed by non-linear least squares fitting using Gaussians where the peak positions and

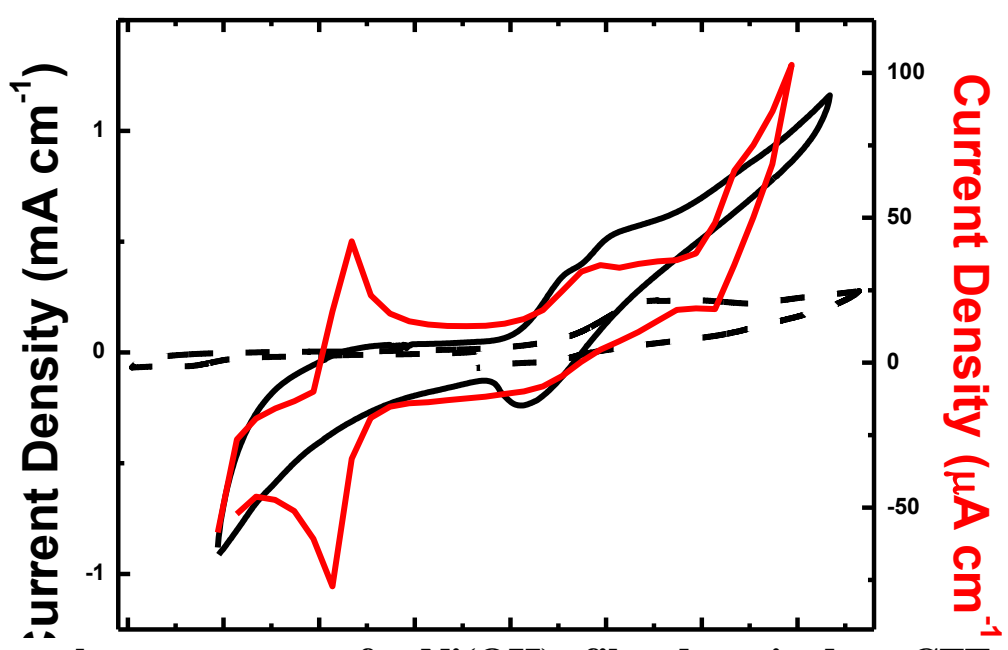

Figure 10.4 Cyclic voltammograms of a $\mathrm{Ni}(\mathrm{OH})_{2}$ film deposited on CFE (black - left yaxis), carbon cloth (black dash - left $\mathrm{y}$-axis), and Ni(II)cyclam in solution (red - right $\mathrm{y}$ axis, working electrode: glassy carbon) in anaerobic $0.1 \mathrm{M} \mathrm{TBAPF}_{6}-\mathrm{CH}_{3} \mathrm{CN}$ solutions (scan rate $25 \mathrm{mV} / \mathrm{s}$ ). 
FWHM were allowed to vary. The Ni $2 \mathrm{p}_{3 / 2}$ and satellite signals for the $23 \mathrm{mM}$ sample film were adequately fit to a single Gaussian each centered at $858.5 \mathrm{eV}$ and $864.3 \mathrm{eV}$, respectively, whereas the observed O 1s signal was found to be comprised of two underlying Gaussians centered at 532.8 $\mathrm{eV}$ and $534.4 \mathrm{eV}$. The $6 \mathrm{mM}$ and $15 \mathrm{mM}$ samples display similar results with $\mathrm{Ni} 2 \mathrm{p}_{3 / 2}$ (satellite) signals at $858.0 \mathrm{eV}(863.9 \mathrm{eV})$ and $856.2 \mathrm{eV}(862.2 \mathrm{eV})$, as well as $\mathrm{O} 1$ s signals at $532.9 \mathrm{eV}$ (with second component at $534.4 \mathrm{eV}$ ) and $531.4 \mathrm{eV}$, respectively.
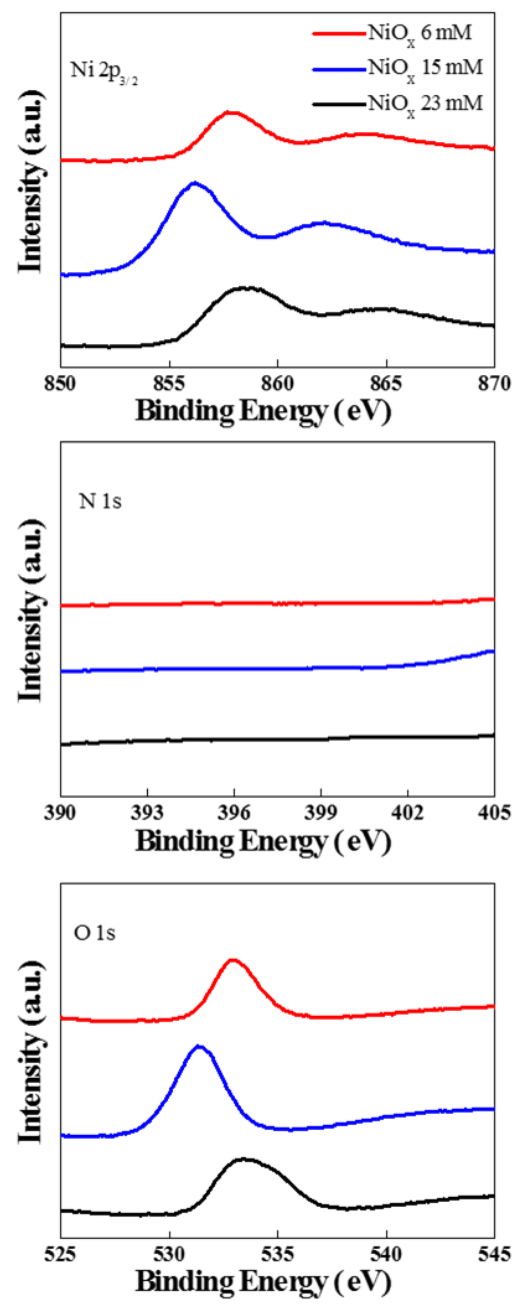

Figure 10.5 X-ray photoelectron spectra of Ni 2p $3 / 2$ (top), $\mathrm{N} 1 \mathrm{~s}$ (middle), and $\mathrm{O}$ 1s (bottom) signals for films prepared from $6 \mathrm{mM}, 15 \mathrm{mM}$, and $23 \mathrm{mM}$ solutions of $\mathrm{Ni}(\mathrm{II})$ cyclam. 
Based on the data above it is proposed that the films are $\mathrm{Ni}(\mathrm{OH})_{2}$ in nature (Singh et al. 2013). XPS signals for $\mathrm{Ni}(\mathrm{OH})_{2}$ are typically $855 \mathrm{eV}-858 \mathrm{eV}$ for the $\mathrm{Ni} 2 \mathrm{p}_{3 / 2}$ and $531 \mathrm{eV}$ for the $\mathrm{O} 1 \mathrm{~s}$ binding energies, which are in agreement with the results found here (vide supra) (Dube et al. 1995, Grosvenor et al. 2006, Mcintyre and Cook 1975). The cause of the O 1s shift to near 533 $\mathrm{eV}$ is uncertain, but may be due to interactions between the $\mathrm{Ni}(\mathrm{OH})_{2}$ nanoparticles and oxygen functional groups on the CFE that may form during the oxidative electrodeposition process under basic conditions. These oxygen functional groups (i.e. carbonyls) on the surface of the CFE may also explain the presence of the second band at $534 \mathrm{eV}$ in the $6 \mathrm{mM}$ and $23 \mathrm{mM}$ preparations.

SEM (with EDS) was also employed to characterize the structure of $\mathrm{Ni}(\mathrm{OH})_{2}$ on carbon cloth. Figure 10.6 shows the FESEM images of $\mathrm{Ni}(\mathrm{OH})_{2}$-coated carbon cloth substrates (Figure 10.6cf). For comparison, FESEM images of the unmodified cloth are also shown (Figure $10.6 \mathrm{a}$ and b). The carbon cloth is composed of large interweaved bunches of microfibers with a diameter of $\sim 15$ $\mu \mathrm{m}$. The figure shows that the surface of unmodified carbon cloth is relatively rough over the size of samples (not all shown here). The SEM images (Figure 10.6 D, e, and f) indicate the morphology of the $\mathrm{Ni}(\mathrm{OH})_{2}$ nanostructures is comprised of a network of intergrown plates. The irregularity of the orientations of the nano-plates suggest that the films may have large surface areas for reactivity. This could open up new opportunities and applications for catalytic $\mathrm{Ni}(\mathrm{OH})_{2}$-based materials. The $\mathrm{Ni}(\mathrm{OH})_{2}$ film prepared with $23 \mathrm{mM} \mathrm{Ni(II)cyclam} \mathrm{appeared} \mathrm{denser} \mathrm{when} \mathrm{compared} \mathrm{to} \mathrm{the} \mathrm{films}$ prepared with lower amounts of the precursor material, suggesting the potential of an increased number of catalytic sites when making films with higher amounts of Ni(II)cyclam. Figure 10.8 presents the ESEM images and EDS spectrums of the unmodified carbon cloth and $\mathrm{Ni}(\mathrm{OH})_{2}$ 
generated from nickel(II) cyclam. All samples displayed EDS peaks corresponding to carbon, resulting from the carbon cloth base. Nickel and oxygen peaks were also present at both sites materials prepared by electrodeposition from nickel(II) cyclam solutions, indicating successful coating of the microfibers. Small amounts of iron were observed in the $\mathrm{Ni}(\mathrm{OH})_{2}$ modified carbon cloth likely due to the contamination during the electrode preparation.
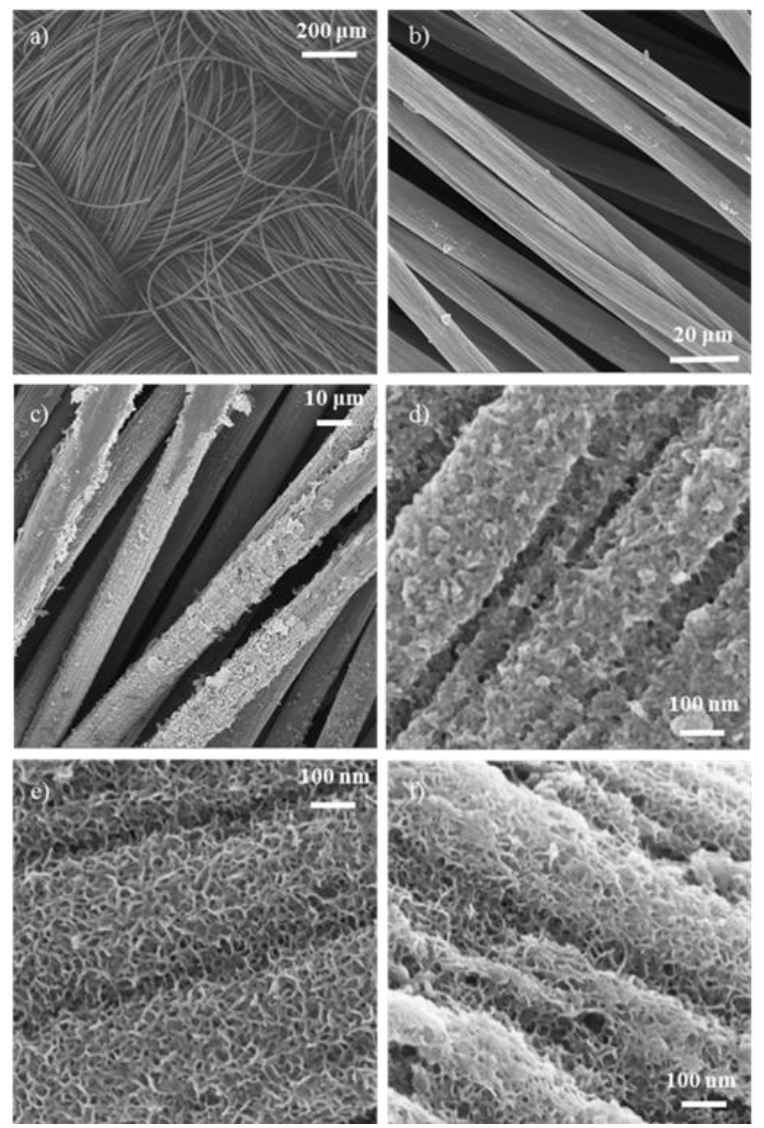

Figure 10.6 SEM images of carbon cloth (a and b) and $\mathrm{Ni}(\mathrm{OH}) 2$-modified carbon cloth that results from electrodeposition from a Ni(II)cyclam precursor solution at concentration of $23 \mathrm{mM}$ (c and d), $15 \mathrm{mM}$ (e) and $6 \mathrm{mM}$ (f).

Tafel plots (log (current density) vs overpotential) for unmodified carbon cloth and $\mathrm{Ni}(\mathrm{OH})_{2}$ coated electrodes generated from nickel(II) cyclam were constructed to identify the rate limiting step of hydrogen production (vida infra) and are shown in Figure 10.7. The unmodified electrode exhibits a Tafel slope of $211 \mathrm{mV}$ decade $^{-1}$ and an exchange current of $0.23 \mathrm{~mA} \mathrm{~cm}^{-2}$. The $\mathrm{Ni}(\mathrm{OH})_{2-}$ 
coated electrodes generated from $\mathrm{Ni}(\mathrm{II}) \mathrm{Cl}_{2} \cdot 6 \mathrm{H}_{2} \mathrm{O}$ showed a Tafel slope of $201 \mathrm{mV}$ decade $^{-1}$. Comparatively, the Tafel analysis of $\mathrm{Ni}(\mathrm{OH})_{2}$-modified electrodes generated from nickel(II) cyclam precursor solutions exhibited Tafel slopes of $105,123,129 \mathrm{mV}$ decade $^{-1}$ when the nickel(II) cyclam concentration in the deposition solution were 23,15 , and $6 \mathrm{mM}$, respectively. The exchange current was $0.34,0.36$, and $0.36 \mathrm{~mA} \mathrm{~cm}^{-2}$, respectively.
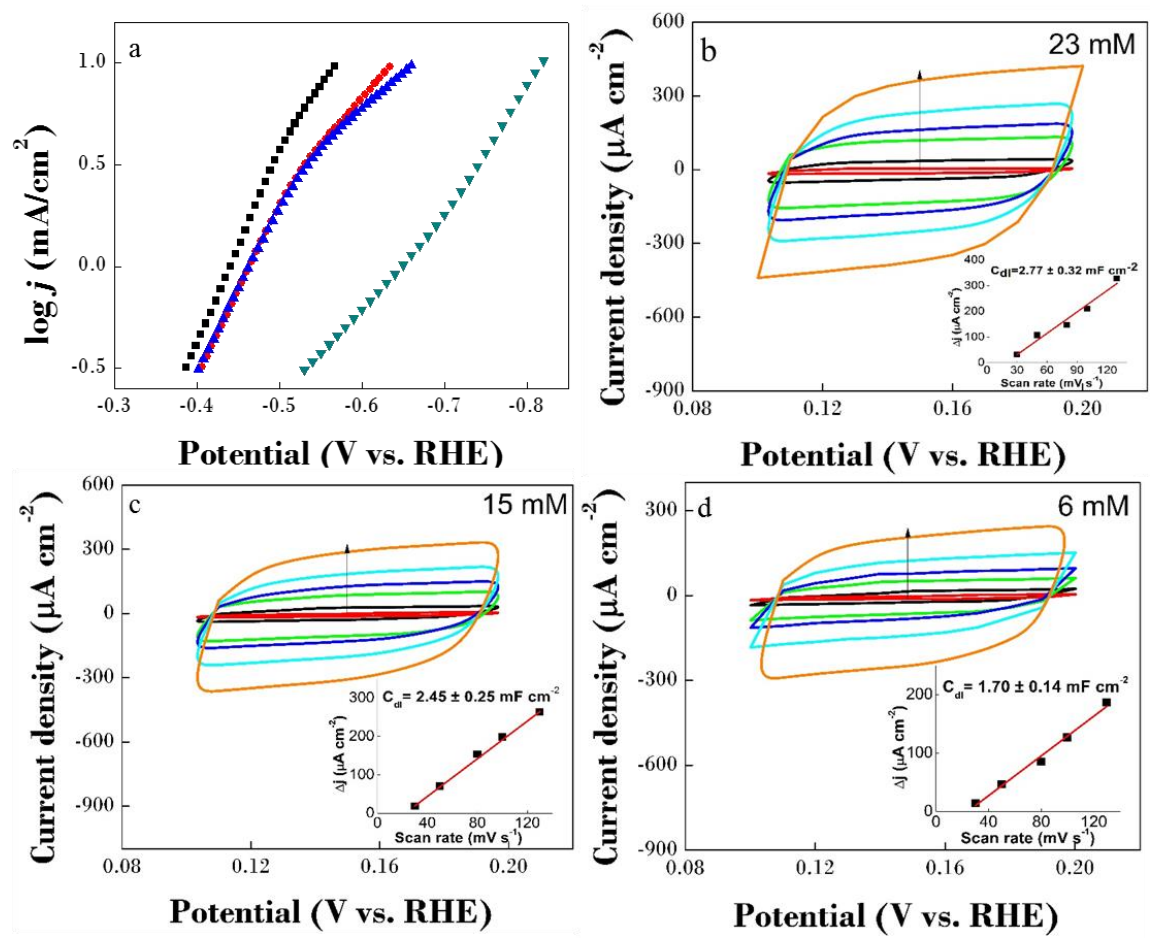

Figure 10.7 a. Tafel slope for carbon cloth (green), $\mathrm{Ni}(\mathrm{OH})_{2}-6 \mathrm{mM}$ precursor (navy), $\mathrm{Ni}(\mathrm{OH})_{2}$ $-15 \mathrm{mM}$ precursor (red) and $\mathrm{Ni}(\mathrm{OH})_{2}-23 \mathrm{mM}$ precursor (black) with a scan rate of $1 \mathrm{mV} \mathrm{s}^{-1}$ measured in PBS buffer $(1 \mathrm{M})$. b-d. CV in the region of 0.1-0.2 V vs. RHE plotted against scan rate and linear regression for the $C_{d l}$ estimation with $23 \mathrm{mM}, 15 \mathrm{mM}$ and $6 \mathrm{mM}$ $\mathrm{Ni}(\mathrm{II})$ cyclam. Scan rate: $130 \mathrm{mV} / \mathrm{s}$ (orange), $100 \mathrm{mV} / \mathrm{s}$ (blue), $80 \mathrm{mV} / \mathrm{s}$ (navy), $50 \mathrm{mV} / \mathrm{s}$ (green), $30 \mathrm{mV} / \mathrm{s}$ (black), and $10 \mathrm{mV} / \mathrm{s}$ (red).

The hydrogen evolution reaction (HER) involves three steps: the Volmer (1), Heyrovsky (2), and Tafel (3) reactions, which involve adsorption of a reduced proton on a surface and chemical recombination to produce $\mathrm{H}_{2}$ as outlined in equations 1 to 3 . 


$$
\begin{gathered}
\mathrm{H}_{3} \mathrm{O}^{+}+e^{-} \rightarrow \mathrm{H}_{a d s}+\mathrm{H}_{2} \mathrm{O} \\
\mathrm{H}_{\text {ads }}+\mathrm{H}_{3} \mathrm{O}^{+}+e^{-} \rightarrow \mathrm{H}_{2}+\mathrm{H}_{2} \mathrm{O} \\
2 \mathrm{H}_{a d s} \rightarrow \mathrm{H}_{2}
\end{gathered}
$$

The expected Tafel slopes for a Volmer, Hyrovsky, or Tafel rate-determining reaction are $120 \mathrm{mV}$ $\operatorname{dec}^{-1}, 40 \mathrm{mV} \mathrm{dec}{ }^{-1}$, and $30 \mathrm{mV} \mathrm{dec}{ }^{-1}$ respectively. Considering the observed Tafel slope of 120 $\mathrm{mV}$ decade $^{-1}$, the rate-determining HER step for the $\mathrm{Ni}(\mathrm{OH})_{2}$ films prepared here is most likely the adsorption related reaction (Volmer).

The active catalytic area was estimated through determination of the electrochemical surface area (ECSA) (Figure $10.7 \mathrm{~b}-\mathrm{d})$. The double layer capacitance $\left(C_{d l}\right)$, which is expected to be linearly proportional to the effective active surface area, can be determined through investigation of the current response of the $\mathrm{Ni}(\mathrm{OH})_{2}$-modified electrodes in a potential region where no Faradaic processes occur. For the $\mathrm{Ni}(\mathrm{OH})_{2}$-modified electrodes, the region of 0.1-0.2 V vs. RHE was used. By plotting the non-Faradaic (charging) current vs. scan rate the $\mathrm{C}_{\mathrm{dl}}$ was quantified. The $C_{d l} \mathrm{~s}$ for the $\mathrm{Ni}(\mathrm{OH})_{2}$-modified electrodes were determined to be $2.77 \pm 0.32 \mathrm{mF} \mathrm{cm}^{-2}, 2.45 \pm 0.25 \mathrm{mF} \mathrm{cm}^{-}$ 2 and $1.70 \pm 0.14 \mathrm{mF} \mathrm{cm}^{-2}$ for electrodes prepared in $23 \mathrm{mM}, 15 \mathrm{mM}$ and $6 \mathrm{mM} \mathrm{Ni(II)cyclam}$ precursor solutions, respectively. The ECSA increased as the starting concentration of $\mathrm{Ni}(\mathrm{II})$ cyclam in the electrodeposition reaction is increased. Assuming that the $C_{d l}$ is directly proportional to the active surface area, these results strongly suggest that a higher concentration of 
$\mathrm{Ni}(\mathrm{II})$ cyclam precursor results in an increased density of catalytically active sites for hydrogen evolution.

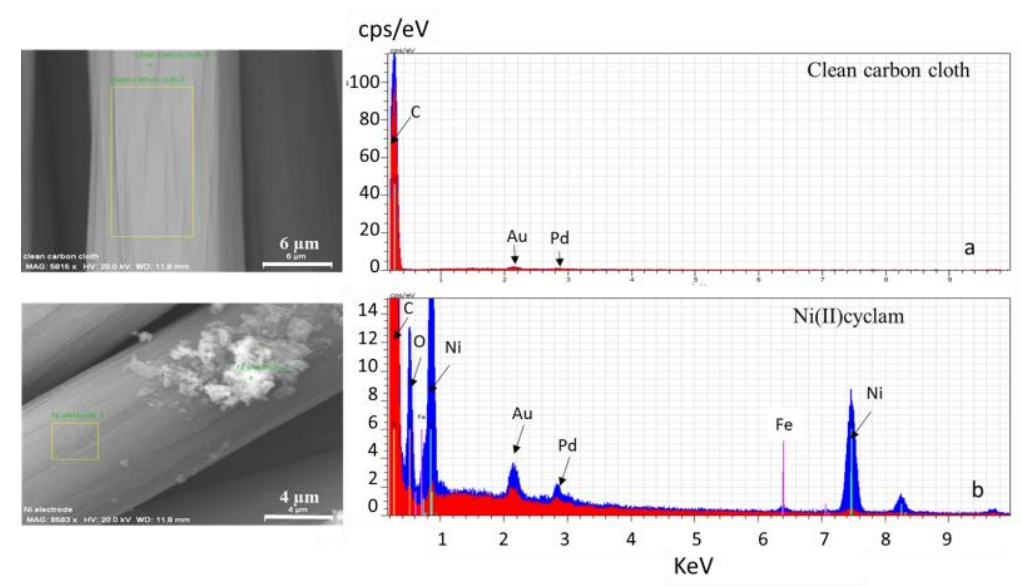

Figure 10.8 EDS spectrums of (a) unmodified carbon cloth, (b) $\mathrm{Ni}(\mathrm{OH}) 2$ modified carbon cloth. Pd and Au peaks results from the metal pre-treatment of the samples prior to SEM collection.

Hydrogen production in an MEC - Hydrogen generation catalyzed by the developed nano-scale $\mathrm{Ni}(\mathrm{OH})_{2}$ films was investigated in an MEC applying a $0.8 \mathrm{~V}$ external voltage. The typical batchprofile current density is shown in Figure 10.9. In general, the decrease in current density observed with operation time is related to substrate consumption. The results are summarized in Table 10.1. Some salient points arise from the analysis of the MEC experiments. First, it is not surprising that the total charge and Coulombic recovery observed for both the $\mathrm{Ni}(\mathrm{OH})_{2}$ film electrodes (regardless of the method of preparation) and the Pt electrode are within statistical error of each other considering these are dependent on the degree of feedstock consumption by the bacterial anodes.

It is noteworthy, then, that the hydrogen recovery efficiencies, $R_{\text {cat, }}$ and overall hydrogen recovery rates, $\mathrm{QH}_{2}$, for $\mathrm{Pt}\left(78.3 \pm 1.9 \%\right.$ and $0.013 \pm 0.001 \mathrm{~m}^{3} \mathrm{H}_{2} \mathrm{~m}^{-2} \mathrm{~d}^{-1}$, respectively) and the $\mathrm{Ni}(\mathrm{OH})_{2}$ film prepared at a higher concentration of $\mathrm{Ni}$ (II)cyclam precursor $(84.3 \pm 3.3 \%$ and $0.014 \pm 0.002$ 
$\mathrm{m}^{3} \mathrm{H}_{2} \mathrm{~m}^{-2} \mathrm{~d}^{-1}$, respectively) are within statistical error. In addition, there is a noticeable difference in the $\mathrm{R}_{\text {cat }}$ and $\mathrm{Q}_{2}$ between the $\mathrm{Ni}(\mathrm{OH})_{2}$ films prepared using $\mathrm{NiCl}_{2}$ (at saturating concentrations, $\mathrm{R}_{\text {cat }}=48.4 \%$ and $\left.\mathrm{QH}_{2}=0.008 \mathrm{~m}^{3} \mathrm{H}_{2} \mathrm{~m}^{-2} \mathrm{~d}^{-1}\right)$ and $\mathrm{Ni}(\mathrm{II})$ cyclam at higher, though well below saturating, concentrations $\left(\mathrm{R}_{\text {cat }}=84.3 \%\right.$ and $\left.\mathrm{Q}_{\mathrm{H} 2}=0.014 \mathrm{~m}^{3} \mathrm{H}_{2} \mathrm{~m}^{-2} \mathrm{~d}^{-1}\right)$. The later results are consistent with a previous study (Selembo et al. 2009). Finally, there appears to be a linear

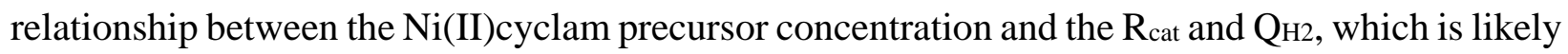
related to an increase in the number of catalytic sites available with films prepared at higher precursor concentration, as supported by the determination of the ESCA.

In summary, a method of preparing $\mathrm{Ni}(\mathrm{OH})_{2}$ films has been demonstrated here by electrophoretic deposition from $\mathrm{Ni}(\mathrm{II})$ cyclam precursor solutions. The films prepared in this manner displayed excellent performance for catalyzing HER for hydrogen production in MECs comparable to Pt. In addition, the MECs employing the $\mathrm{Ni}(\mathrm{OH})_{2}$ catalyst exhibited stable hydrogen production for one month, therefore, making these Ni-based materials stable electrodes in MECs.

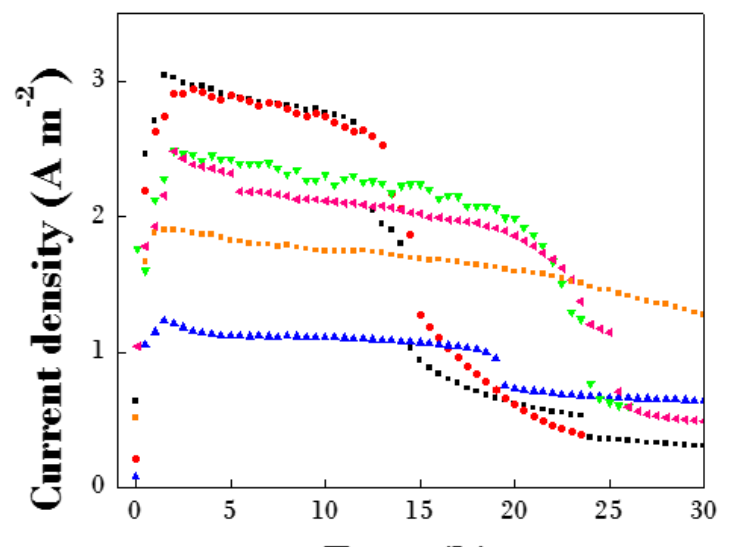

Time (h)

Figure 10.9 Current density of the MEC with modified carbon cloth with $\mathrm{Ni}(\mathrm{OH}) 2(23 \mathrm{mM}$ $\mathrm{Ni}(\mathrm{II})$ cyclam (black), $15 \mathrm{mM}$ (green), and $6 \mathrm{mM}$ (pink)), $\mathrm{Ni}(\mathrm{OH}) 2$ from $\mathrm{Ni}(\mathrm{II}) \mathrm{Cl} 2 \cdot 6 \mathrm{H} 2 \mathrm{O}$ (orange) or Pt (red) and unmodified carbon cloth (blue) at an applied voltage of $0.8 \mathrm{~V}$. 
Table 10.1 Hydrogen production and efficiencies of the MECs with modified carbon cloth with nickel hydroxide or Pt and unmodified carbon cloth at an applied voltage of $0.8 \mathrm{~V}$

\begin{tabular}{|c|c|c|c|c|c|c|}
\hline & & $\mathrm{C}_{\text {total }}{ }^{\mathrm{a}}, \mathrm{C}$ & $\mathrm{C}_{\mathrm{R}}^{\mathrm{b}}, \%$ & $\mathrm{R}_{\text {cat }}{ }^{\mathrm{c}}, \%$ & $\mathrm{R}_{\mathrm{H} 2}{ }^{\mathrm{d}}, \%$ & $\begin{array}{c}\mathrm{Q}_{\mathrm{H}_{2}}{ }^{\mathrm{e}}, \mathrm{m}^{3} \mathrm{H}_{2} \\
\mathrm{~m}^{-2} \mathrm{~d}^{-1}\end{array}$ \\
\hline \multirow{3}{*}{ Ni(II)cyclam } & $23 \mathrm{mM}$ & $\begin{array}{c}71.6 \pm \\
2.1 \\
\end{array}$ & $25.0 \pm 3.4$ & $84.3 \pm 3.3$ & $\begin{array}{c}20.7 \pm \\
1.1\end{array}$ & $0.014 \pm 0.002$ \\
\hline & $15 \mathrm{mM}$ & $\begin{array}{c}75.5 \pm \\
2.9 \\
\end{array}$ & $27.6 \pm 4.7$ & $69.6 \pm 4.0$ & $\begin{array}{c}19.2 \pm \\
1.9 \\
\end{array}$ & $0.012 \pm 0.003$ \\
\hline & $6 \mathrm{mM}$ & $\begin{array}{c}75.4 \pm \\
2.1 \\
\end{array}$ & $26.4 \pm 3.1$ & $59.2 \pm 2.1$ & $\begin{array}{c}15.7 \pm \\
1.3 \\
\end{array}$ & $0.010 \pm 0.001$ \\
\hline $\mathrm{Ni}(\mathrm{II}) \mathrm{Cl}_{2} \bullet 6 \mathrm{H}_{2} \mathrm{O}$ & & $\begin{array}{c}71.1 \pm \\
0.8 \\
\end{array}$ & $22.3 \pm 4.0$ & $48.4 \pm 4.2$ & $\begin{array}{c}10.8 \pm \\
2.1 \\
\end{array}$ & $0.008 \pm 0.002$ \\
\hline $\mathrm{Pt}$ & & $\begin{array}{c}71.4 \pm \\
1.2 \\
\end{array}$ & $26.2 \pm 2.4$ & $78.3 \pm 1.9$ & $\begin{array}{c}19.1 \pm \\
0.9\end{array}$ & $0.013 \pm 0.001$ \\
\hline Carbon cloth & & $\begin{array}{c}40.1 \pm \\
1.0\end{array}$ & $10.2 \pm 1.9$ & $21.1 \pm 3.8$ & $2.1 \pm 1.4$ & $0.002 \pm 0.001$ \\
\hline
\end{tabular}

a, Total coulombs passed - reports on the complete consumption of the electron source

(organics - acetate) in the anode chamber and is expected to be similar for all catalytic cathodes;

b, Coulombic recovery; c, Cathodic hydrogen recovery; d, Overall hydrogen recovery; e,

Hydrogen production rate. All were calculated according to the equation in experimental part. 


\section{Chapter 11 Perspectives}

The cooperation of BES and FO creates an innovative treatment system, which takes advantage of both technologies and can accomplish the recovery of valuable resources such as energy, water, and nutrient from various wastewaters. The cooperation, through either internal or external connection, also generates a strong synergy between BES and FO and helps overcome some inherent issues of each technology. The research of OsBES is still in an infancy stage, but great promise in resource recovery and progress of both BES and FO as individual technology will accelerate the development of the OsBES technology. More efforts must be invested to identify application niches, understand energy issues, alleviate membrane fouling, and scale up OsBES to a transitional stage.

\section{Energy balance}

Integrated system needs to take energy consumption and balance into consideration since more units are included in the system. Besides, the energy performance has not been widely presented in the BES studies. A new parameter, normalized energy recovery (NER), has been proposed to present energy consumption and production. NER is expressed in either $\mathrm{kWh} \mathrm{m}^{-3}$ or $\mathrm{kWh}$ $\mathrm{kgCOD}^{-1}$. Like BES studies, many OsBES studies do not report energy data; only power density data are presented. To better understand BES/OsBES performance and identify their application niches, energy data must be included (He 2017)

\section{Potential applications}

An OsBES may be applied to recover valuable resources with simultaneous treatment of various wastewaters including both low strength (e.g., municipal wastewater) and high strength (e.g., side stream, food/beverage wastewater, livestock wastewater, and landfill leachate). However, the 
strategies and goal of the applications will differ, because of different characteristics of wastewaters. The application to treat low strength wastewater will need to focus on energyefficient treatment and water recovery. Energy recovery from low strength wastewater is still important, but its primary goal will be to help achieve energy-neutral treatment. Nutrient recovery may not be feasible, due to very low concentrations of both nitrogen and phosphorus in low strength wastewater. High-strength wastewater contains high concentrations of organic matters and nutrients, and will be suitable for simultaneous NEW recovery.

\section{Coordination between MEC and FO}

The application of the MEC-FO system will need to address a key issue of system coordination between the MEC unit and FO unit. MEC is a biological process and tends to be slow, while FO is a physical process and usually fast. The difference in the treatment speed between the two creates the issue of coordinating the treatment capacities. In MEC-FO system, the HRT of an MEC that was used for energy and ammonia recovery was $48 \mathrm{~h}$ while the FO unit required only $5 \mathrm{~h}$ to complete water recovery. As a result, the FO unit was on standby most of the operation time and its treatment capacity would be wasted. Such discrepancy may be solved by increasing the treatment speed of the MEC unit (e.g., through optimizing MEC operation and materials), or by reducing the size of the FO unit so that a continuous supply of MEC effluent can be achieved.

\section{Membrane fouling}

Although membrane fouling in an FO process is generally less severe than that in pressure-driven membrane processes, it is still an inevitable phenomenon in an OsBES, and can lead to an additional hydraulic resistance that reduces osmotic pressure and water recovery, thereby increasing the capital and operational costs. When treating wastewater, the organic fouling due to the presence of organic matters and microorganisms can become significant (Zhao et al. 2011), 
and in this aspect, a coupled OsBES may be more advantageous over an integrated OsBES, because the BES unit in the coupled OsBES acts as a pre-treatment step and reduces organic contents in the liquid stream before it enters the FO unit. When fouling occurs, backwash is a commonly used method to remove foulants and retrieve the FO performance. Membrane fouling in FO can be almost fully reversible by backwash, due to the loose fouling layer on the membrane surface in the absence of external pressure (Lee et al. 2010). More severe fouling would require chemical cleaning, either in situ or ex situ. No matter which cleaning method, the microbial community in the anode of an integrated OsBES would be adversely affected, for example by a shock from a high salinity solution or chemical toxicity. To address this problem, membrane fabrication and modification must be advanced to reduce membrane fouling and cleaning requirement. Fouling alters the membrane properties and in some cases, it may benefit energy recovery in an OsBES. It was found that the current generation of an OsMFC was increased by 34\% upon membrane fouling without water flux (Zhu et al. 2016). This is because that the fouled FO membrane showed significantly higher flux of protons and other ions than a pristine membrane, resulting in a lower internal resistance and higher current generation.

\section{System scaling up}

Development of the OsBES technology must advance system scaling up, and this will be built on the ongoing scaling up efforts for both BES and FO. Scaling up FO is relatively easy and straightforward, because of its simple structure. There have been several pilot FO systems developed for extracting water from domestic wastewater, fracking wastewater and mine-impaired groundwater (Hancock et al. 2011b, McGinnis et al. 2013, Phuntsho et al. 2016). Thus, the key challenge to scaling up an OsBES will lie in the BES part. There have been a number of reports of BES scaling up, such as MFCs, MECs and MDCs (Cusick et al. 2011, Feng et al. 2014, Wu et al. 
2016, Zhang and He 2015), and those will provide valuable experiences to scaling up OsBES. An effective step to transform laboratory systems to pilot/full systems is to establish a transitionalscale system, which has a significantly larger size than bench systems but smaller than pilot/full systems. Such a transitional system possesses some features of both bench and pilot/full systems, and can be a great platform for further research. For example, a 200-L MFC system was developed as a transitional-scale system (much larger than bench MFCs that are usually no more than $1 \mathrm{~L}$ but smaller than typical pilot systems of >3,000 L) for treating domestic wastewater (Ge et al. 2015). Scaling up the integrated OsBES will largely benefit from BES scaling up efforts. Mathematical modeling can be a powerful tool to help develop transitional-scale systems based on the results of bench systems (Luo et al. 2016). A key objective for studying the transitional-scale systems is to perform techno-economic analysis, which will reveal both technical and economical feasibility of the OsBES technology. Involving both membranes and electrodes in an OsBES can result in a high capital cost; however, if an appropriate application niche is identified, there could be the potential need for this technology. In the previously mentioned study of 200-L MFC system, the initial analysis of the capital cost indicated that this system could be more competitive in the market of small-scale wastewater treatment (e.g., $<40 \mathrm{~m}^{3}$ day $^{-1}$ ) (Ge et al. 2015). Rapid progress in material science will lead to the reduction of the cost associated with membranes and electrodes. 


\section{Reference}

Achilli, A., Cath, T.Y. and Childress, A.E. (2010) Selection of inorganic-based draw solutions for forward osmosis applications. Journal of Membrane Science 364(1), 233-241.

Achilli, A., Cath, T.Y., Marchand, E.A. and Childress, A.E. (2009) The forward osmosis membrane bioreactor: a low fouling alternative to MBR processes. Desalination 239(1), $10-21$.

Achilli, A. and Childress, A.E. (2010) Pressure retarded osmosis: From the vision of Sidney Loeb to the first prototype installation-Review. Desalination 261(3), 205-211.

Aeshala, L., Uppaluri, R. and Verma, A. (2013) Effect of cationic and anionic solid polymer electrolyte on direct electrochemical reduction of gaseous $\mathrm{CO}_{2}$ to fuel. J. $\mathrm{CO}_{2}$ Util. 3, 4955.

Ahn, Y.-H. (2006a) Sustainable nitrogen elimination biotechnologies: a review. Process Biochem. 41(8), 1709-1721.

Ahn, Y.H. (2006b) Sustainable nitrogen elimination biotechnologies: a review. Process Biochem. 41(8), 1709-1721.

Akther, N., Sodiq, A., Giwa, A., Daer, S., Arafat, H. and Hasan, S. (2015) Recent advancements in forward osmosis desalination: a review. Chem. Eng. J. 281, 502-522.

Alturki, A., McDonald, J., Khan, S.J., Hai, F.I., Price, W.E. and Nghiem, L.D. (2012) Performance of a novel osmotic membrane bioreactor (OMBR) system: flux stability and removal of trace organics. Bioresour. Technol. 113, 201-206.

An, J. and Lee, H.S. (2014) Occurrence and Implications of Voltage Reversal in Stacked Microbial Fuel Cells. ChemSusChem 7(6), 1689-1695.

Angenent, L.T. and Sung, S. (2001) Development of anaerobic migrating blanket reactor (AMBR), a novel anaerobic treatment system. Water Res. 35(7), 1739-1747.

Ansari, A.J., Hai, F.I., Price, W.E., Drewes, J.E. and Nghiem, L.D. (2017) Forward osmosis as a platform for resource recovery from municipal wastewater-A critical assessment of the literature. J. Membr. Sci. 529, 195-206.

Arena, J.T., Chwatko, M., Robillard, H.A. and McCutcheon, J.R. (2015) pH sensitivity of ion exchange through a thin film composite membrane in forward osmosis. Environ. Sci. Technol. Lett. 2(7), 177-182.

Arena, J.T., Manickam, S.S., Reimund, K.K., Freeman, B.D. and McCutcheon, J.R. (2014) Solute and water transport in forward osmosis using polydopamine modified thin film composite membranes. Desalination 343, 8-16.

Arredondo, M.R., Kuntke, P., Jeremiasse, A., Sleutels, T., Buisman, C. and ter Heijne, A. (2015) Bioelectrochemical systems for nitrogen removal and recovery from wastewater. Environ. Sci.: Water Res. Technol. 1(1), 22-33. 
Bani-Melhem, K. and Elektorowicz, M. (2010) Development of a novel submerged membrane electro-bioreactor (SMEBR): performance for fouling reduction. Environ. Sci. Technol. 44(9), 3298-3304.

Barker, D.J. and Stuckey, D.C. (1999) A review of soluble microbial products (SMP) in wastewater treatment systems. Water research 33(14), 3063-3082.

Batstone, D., Hülsen, T., Mehta, C. and Keller, J. (2015) Platforms for energy and nutrient recovery from domestic wastewater: A review. Chemosphere 140, 2-11.

Baun, D.L. and Christensen, T.H. (2004) Speciation of heavy metals in landfill leachate: a review. Waste management \& research 22(1), 3-23.

Beley, M., Collin, J.P., Ruppert, R. and Sauvage, J.P. (1986) Electrocatalytic reduction of carbon dioxide by nickel cyclam $2+$ in water: study of the factors affecting the efficiency and the selectivity of the process. J. Am. Chem. Soc. 108(24), 7461-7467.

Bicer, Y., Dincer, I., Zamfirescu, C., Vezina, G. and Raso, F. (2016) Comparative Life Cycle Assessment of Various Ammonia Production Methods. J Clean Prod. 135, 1379-1395.

Boo, C., Elimelech, M. and Hong, S. (2013) Fouling control in a forward osmosis process integrating seawater desalination and wastewater reclamation. J. Membr. Sci. 444, 148156.

Boo, C., Lee, S., Elimelech, M., Meng, Z. and Hong, S. (2012) Colloidal fouling in forward osmosis: role of reverse salt diffusion. J. Membr. Sci. 390, 277-284.

Bosnich, B., Tobe, M.L. and Webb, G.A. (1965) Complexes of Nickel(II)with a Cyclic Tetradentate Secondary Amine. Inorg Chem 4(8), 1109-1112.

Cao, X., Huang, X., Liang, P., Xiao, K., Zhou, Y., Zhang, X. and Logan, B.E. (2009) A new method for water desalination using microbial desalination cells. Environ. Sci. Technol. 43(18), 7148-7152.

Capodaglio, A.G., Hlavínek, P. and Raboni, M. (2015) Physico-chemical technologies for nitrogen removal from wastewaters: a review. Revista Ambiente \& Agua 10(3), 481-498.

Cath, T.Y., Adams, D. and Childress, A.E. (2005a) Membrane contactor processes for wastewater reclamation in space: II. Combined direct osmosis, osmotic distillation, and membrane distillation for treatment of metabolic wastewater. J. Membr. Sci. 257(1), 111-119.

Cath, T.Y., Childress, A.E. and Elimelech, M. (2006) Forward osmosis: principles, applications, and recent developments. Journal of membrane science 281(1), 70-87.

Cath, T.Y., Elimelech, M., McCutcheon, J.R., McGinnis, R.L., Achilli, A., Anastasio, D., Brady, A.R., Childress, A.E., Farr, I.V. and Hancock, N.T. (2013) Standard methodology for evaluating membrane performance in osmotically driven membrane processes. Desalination 312, 31-38.

Cath, T.Y., Gormly, S., Beaudry, E.G., Flynn, M.T., Adams, V.D. and Childress, A.E. (2005b) Membrane contactor processes for wastewater reclamation in space: Part I. Direct osmotic concentration as pretreatment for reverse osmosis. J. Membr. Sci. 257(1), 85-98. 
Chanukya, B., Patil, S. and Rastogi, N.K. (2013) Influence of concentration polarization on flux behavior in forward osmosis during desalination using ammonium bicarbonate. Desalination 312, 39-44.

Chekli, L., Phuntsho, S., Shon, H.K., Vigneswaran, S., Kandasamy, J. and Chanan, A. (2012) A review of draw solutes in forward osmosis process and their use in modern applications. Desalin. Water. Treat. 43(1-3), 167-184.

Chen, H. and Ruckenstein, E. (2015) Hydrated Ions: From Individual Ions to Ion Pairs to Ion Clusters. J. Phys. Chem. B 119(39), 12671-12676.

Chen, J., Yang, C., Zhou, J. and Wang, X. (2007) Study of the influence of the electric field on membrane flux of a new type of membrane bioreactor. Chem. Eng. J. 128(2), 177-180.

Chen, S., Liu, G., Zhang, R., Qin, B. and Luo, Y. (2012) Development of the microbial electrolysis desalination and chemical-production cell for desalination as well as acid and alkali productions. Environ. Sci. Technol. 46(4), 2467-2472.

Chen, X., Liang, P., Zhang, X. and Huang, X. (2016) Bioelectrochemical systems-driven directional ion transport enables low-energy water desalination, pollutant removal, and resource recovery. Bioresour. Technol. 215, 274-284.

Cheng, K.Y., Kaksonen, A.H. and Cord-Ruwisch, R. (2013) Ammonia recycling enables sustainable operation of bioelectrochemical systems. Bioresour. Technol. 143, 25-31.

Cheng, S., Xing, D., Call, D.F. and Logan, B.E. (2009) Direct biological conversion of electrical current into methane by electromethanogenesis. Environ. Sci. Technol. 43(10), 3953-3958.

Chung, T.-S., Zhang, S., Wang, K.Y., Su, J. and Ling, M.M. (2012a) Forward osmosis processes: Yesterday, today and tomorrow. Desalination 287, 78-81.

Chung, T.S., Zhang, S., Wang, K.Y., Su, J. and Ling, M.M. (2012b) Forward osmosis processes: yesterday, today and tomorrow. Desalination 287, 78-81.

Clauwaert, P., Mulenga, S., Aelterman, P. and Verstraete, W. (2009) Litre-scale microbial fuel cells operated in a complete loop. Appl. Microbiol. Biotechnol. 83(2), 241.

Clauwaert, P., Rabaey, K., Aelterman, P., De Schamphelaire, L., Pham, T.H., Boeckx, P., Boon, N. and Verstraete, W. (2007) Biological denitrification in microbial fuel cells. Environ. Sci. Technol. 41(9), 3354-3360.

Coday, B.D., Xu, P., Beaudry, E.G., Herron, J., Lampi, K., Hancock, N.T. and Cath, T.Y. (2014) The sweet spot of forward osmosis: Treatment of produced water, drilling wastewater, and other complex and difficult liquid streams. Desalination 333(1), 23-35.

Cord-Ruwisch, R., Law, Y. and Cheng, K.Y. (2011) Ammonium as a sustainable proton shuttle in bioelectrochemical systems. Bioresource technology 102(20), 9691-9696.

Cordell, D., Drangert, J.O. and White, S. (2009) The story of phosphorus: global food security and food for thought. Global Environ. Change 19(2), 292-305.

Cornelissen, E., Harmsen, D., Dekorte, K., Ruiken, C., Qin, J., Oo, H. and Wessels, L. (2008) Membrane fouling and process performance of forward osmosis membranes on activated sludge. J. Membr. Sci. 319(1-2), 158-168. 
Cornelissen, E.R., Harmsen, D., Beerendonk, E.F., Qin, J.J., Oo, H., de Korte, K.F. and Kappelhof, J.W. (2011) The innovative osmotic membrane bioreactor (OMBR) for reuse of wastewater. Water Sci. Technol. 63(8), 1557-1565.

Cusick, R.D., Bryan, B., Parker, D.S., Merrill, M.D., Mehanna, M., Kiely, P.D., Liu, G. and Logan, B.E. (2011) Performance of a pilot-scale continuous flow microbial electrolysis cell fed winery wastewater. Appl. Microbiol. Biotechnol. 89(6), 2053-2063.

Cusick, R.D., Kim, Y. and Logan, B.E. (2012) Energy capture from thermolytic solutions in microbial reverse-electrodialysis cells. Science 335(6075), 1474-1477.

Damiano, L., Jambeck, J.R. and Ringelberg, D.B. (2014) Municipal solid waste landfill leachate treatment and electricity production using microbial fuel cells. Appl. Biochem. Biotechnol. 173(2), 472-485.

Dasgupta, P.K. and Dong, S. (1986) Solubility of ammonia in liquid water and generation of trace levels of standard gaseous ammonia. Atmos. Environ. 20(3), 565-570.

de Fraiture, C., Wichelns, D., Rockstrom, J., Kemp-Benedict, E., Eriyagama, N., Gordon, L.J., Hanjra, M.A., Hoogeveen, J., Huber-Lee, A. and Karlberg, L. (2007) Looking ahead to 2050: scenarios of alternative investment approaches, International Water Management Institute.

Desloover, J., Abate Woldeyohannis, A., Verstraete, W., Boon, N. and Rabaey, K. (2012) Electrochemical resource recovery from digestate to prevent ammonia toxicity during anaerobic digestion. Environ. Sci. Technol. 46(21), 12209-12216.

Ding, Y.C., Yuan, J.L. and Chang, Z.R. (1997) Cyclic voltammetry response of coprecipitated $\mathrm{Ni}(\mathrm{OH})(2)$ electrode in $5 \mathrm{M} \mathrm{KOH}$ solution. J. Power Sources 69(1-2), 47-54.

Dodds, W.K., Bouska, W.W., Eitzmann, J.L., Pilger, T.J., Pitts, K.L., Riley, A.J., Schloesser, J.T. and Thornbrugh, D.J. (2008) Eutrophication of US freshwaters: analysis of potential economic damages, ACS Publications.

Dong, Y., Feng, Y., Qu, Y., Du, Y., Zhou, X. and Liu, J. (2015) A combined system of microbial fuel cell and intermittently aerated biological filter for energy self-sufficient wastewater treatment. Sci. Rep. 5.

Dube, C.E., Workie, B., Kounaves, S.P., Robbat, A., Aksu, M.L. and Davies, G. (1995) Electrodeposition of Metal Alloy and Mixed-Oxide Films Using a Single-Precursor Tetranuclear Copper-Nickel Complex. J. Electrochem. Soc. 142(10), 3357-3365.

Dykstra, J., Biesheuvel, P., Bruning, H. and Ter Heijne, A. (2014a) Theory of ion transport with fast acid-base equilibrations in bioelectrochemical systems. Phys. Rev. E 90(1), 013302.

Dykstra, J.E., Biesheuvel, P.M., Bruning, H. and Ter Heijne, A. (2014b) Theory of ion transport with fast acid-base equilibrations in bioelectrochemical systems. Physical Review E 90(1).

Dykstra, J.E., Biesheuvel, P.M., Bruning, H. and Ter Heijne, A. (2014c) Theory of ion transport with fast acid-base equilibrations in bioelectrochemical systems. Phys. Rev. E 90(1), 013302.

ElMekawy, A., Hegab, H.M. and Pant, D. (2014) The near-future integration of microbial desalination cells with reverse osmosis technology. Energ. Environ. Sci. 7(12), 3921-3933. 
Erisman, J.W., Sutton, M.A., Galloway, J., Klimont, Z. and Winiwarter, W. (2008) How a century of ammonia synthesis changed the world. Nat. Geosci. 1(10), 636-639.

Fan, Y., Sharbrough, E. and Liu, H. (2008) Quantification of the internal resistance distribution of microbial fuel cells. Environ. Sci. Technol. 42(21), 8101-8107.

Feng, Y., He, W., Liu, J., Wang, X., Qu, Y. and Ren, N. (2014) A horizontal plug flow and stackable pilot microbial fuel cell for municipal wastewater treatment. Bioresour. Technol. $156,132-138$.

Foo, K. and Hameed, B. (2009) An overview of landfill leachate treatment via activated carbon adsorption process. J. Hazard. Mater. 171(1), 54-60.

Galama, A., Post, J., Stuart, M.C. and Biesheuvel, P. (2013) Validity of the Boltzmann equation to describe Donnan equilibrium at the membrane-solution interface. J. Membr. Sci. 442, 131-139.

Galama, A., Vermaas, D., Veerman, J., Saakes, M., Rijnaarts, H., Post, J. and Nijmeijer, K. (2014) Membrane resistance: The effect of salinity gradients over a cation exchange membrane. J. Membr. Sci. 467, 279-291.

Gálvez, A., Greenman, J. and Ieropoulos, I. (2009) Landfill leachate treatment with microbial fuel cells; scale-up through plurality. Bioresour. Technol. 100(21), 5085-5091.

Ganesh, K. and Jambeck, J.R. (2013) Treatment of landfill leachate using microbial fuel cells: Alternative anodes and semi-continuous operation. Bioresour. Technol. 139, 383-387.

Gao, Y., Li, W., Lay, W.C., Coster, H.G., Fane, A.G. and Tang, C.Y. (2013) Characterization of forward osmosis membranes by electrochemical impedance spectroscopy. Desalination $312,45-51$.

Ge, Z. and He, Z. (2012) Effects of draw solutions and membrane conditions on electricity generation and water flux in osmotic microbial fuel cells. Bioresour. Technol. 109, 70-76.

Ge, Z., Li, J., Xiao, L., Tong, Y. and He, Z. (2013a) Recovery of electrical energy in microbial fuel cells: brief review. Environ. Sci. Technol. Lett. 1(2), 137-141.

Ge, Z., Ping, Q., Xiao, L. and He, Z. (2013b) Reducing effluent discharge and recovering bioenergy in an osmotic microbial fuel cell treating domestic wastewater. Desalination 312, 52-59.

Ge, Z., Wu, L., Zhang, F. and He, Z. (2015) Energy extraction from a large-scale microbial fuel cell system treating municipal wastewater. J. Power Sources 297, 260-264.

Geise, G.M., Curtis, A.J., Hatzell, M.C., Hickner, M.A. and Logan, B.E. (2013) Salt concentration differences alter membrane resistance in reverse electrodialysis stacks. Environ. Sci. Technol. Lett. 1(1), 36-39.

Gotvajn, A.Ž., Tišler, T. and Zagorc-Končan, J. (2009) Comparison of different treatment strategies for industrial landfill leachate. J. Hazard. Mater. 162(2), 1446-1456.

Grant, S.B., Saphores, J.-D., Feldman, D.L., Hamilton, A.J., Fletcher, T.D., Cook, P.L., Stewardson, M., Sanders, B.F., Levin, L.A. and Ambrose, R.F. (2012) Taking the "waste" out of "wastewater" for human water security and ecosystem sustainability. Science 337(6095), 681-686. 
Greenman, J., Gálvez, A., Giusti, L. and Ieropoulos, I. (2009) Electricity from landfill leachate using microbial fuel cells: comparison with a biological aerated filter. Enzyme Microb. Technol. 44(2), 112-119.

Grosvenor, A.P., Biesinger, M.C., Smart, R.S. and McIntyre, N.S. (2006) New interpretations of XPS spectra of nickel metal and oxides. Surf. Sci. 600(9), 1771-1779.

Gupta, V., Sadegh, H., Yari, M., Shahryari Ghoshekandi, R., Maazinejad, B. and Chahardori, M. (2015) Removal of ammonium ions from wastewater: A short review in development of efficient methods. Global J. Environ. Sci. Manage. 1(2), 149-158.

Haddadi, S., Elbeshbishy, E. and Lee, H.-S. (2013) Implication of diffusion and significance of anodic $\mathrm{pH}$ in nitrogen-recovering microbial electrochemical cells. Bioresour. Technol. $142,562-569$.

Haddadi, S., Nabi-Bidhendi, G. and Mehrdadi, N. (2014) Nitrogen removal from wastewater through microbial electrolysis cells and cation exchange membrane. J. Environ. Health. Sci. Eng. 12(1), 48.

Hamelers, H.V., Ter Heijne, A., Sleutels, T.H., Jeremiasse, A.W., Strik, D.P. and Buisman, C.J. (2010) New applications and performance of bioelectrochemical systems. Appl. Microbiol. Biotechnol. 85(6), 1673-1685.

Hancock, N.T., Phillip, W.A., Elimelech, M. and Cath, T.Y. (2011a) Bidirectional permeation of electrolytes in osmotically driven membrane processes. Environ. Sci. Technol. 45(24), 10642-10651.

Hancock, N.T., Xu, P., Heil, D.M., Bellona, C. and Cath, T.Y. (2011b) Comprehensive bench-and pilot-scale investigation of trace organic compounds rejection by forward osmosis. Environ. Sci. Technol. 45(19), 8483-8490.

Harnisch, F. and Schröder, U. (2009) Selectivity versus mobility: separation of anode and cathode in microbial bioelectrochemical systems. ChemSusChem 2(10), 921-926.

He, Z. (2017) Development of Microbial Fuel Cells Needs To Go beyond "Power Density". ACS Energy Lett. 2, 700-702.

He, Z., Huang, Y., Manohar, A.K. and Mansfeld, F. (2008) Effect of electrolyte pH on the rate of the anodic and cathodic reactions in an air-cathode microbial fuel cell. Bioelectrochemistry 74(1), 78-82.

Higa, M., Tanioka, A. and Kira, A. (1998) A novel measurement method of Donnan potential at an interface between a charged membrane and mixed salt solution. J. Membr. Sci. 140(2), 213-220.

Hoek, E.M. and Elimelech, M. (2003) Cake-enhanced concentration polarization: a new fouling mechanism for salt-rejecting membranes. Environ. Sci. Technol. 37(24), 5581-5588.

Hoffert, M.I., Caldeira, K., Benford, G., Criswell, D.R., Green, C., Herzog, H., Jain, A.K., Kheshgi, H.S., Lackner, K.S. and Lewis, J.S. (2002) Advanced technology paths to global climate stability: energy for a greenhouse planet. Science 298(5595), 981-987.

Holloway, R.W., Childress, A.E., Dennett, K.E. and Cath, T.Y. (2007) Forward osmosis for concentration of anaerobic digester centrate. Water Res. 41(17), 4005-4014. 
Holloway, R.W., Maltos, R., Vanneste, J. and Cath, T.Y. (2015) Mixed draw solutions for improved forward osmosis performance. J. Membr. Sci. 491, 121-131.

Hoornweg, D. and Bhada-Tata, P. (2012) What a waste: a global review of solid waste management.

Hou, D., Lu, L. and Ren, Z.J. (2016) Microbial fuel cells and osmotic membrane bioreactors have mutual benefits for wastewater treatment and energy production. Water Res. 98, 183-189.

Howarth, R.W., Billen, G., Swaney, D., Townsend, A., Jaworski, N., Lajtha, K., Downing, J.A., Elmgren, R., Caraco, N. and Jordan, T. (1996) Nitrogen cycling in the North Atlantic Ocean and its watersheds, pp. 75-139, Springer.

Hu, H., Fan, Y. and Liu, H. (2009) Hydrogen production in single-chamber tubular microbial electrolysis cells using non-precious-metal catalysts. Int. J. Hydrogen Energy 34(20), 8535-8542.

Huang, Y.-X., Liu, X.-W., Sun, X.-F., Sheng, G.-P., Zhang, Y.-Y., Yan, G.-M., Wang, S.-G., Xu, A.-W. and Yu, H.-Q. (2011) A new cathodic electrode deposit with palladium nanoparticles for cost-effective hydrogen production in a microbial electrolysis cell. Int. J. Hydrogen Energy 36(4), 2773-2776.

Ismail, Z.Z. and Ibrahim, M.A. (2015) Desalination of oilfield produced water associated with treatment of domestic wastewater and bioelectricity generation in microbial osmotic fuel cell. J. Membr. Sci. 490, 247-255.

Jadhav, G.S. and Ghangrekar, M.M. (2009) Performance of microbial fuel cell subjected to variation in $\mathrm{pH}$, temperature, external load and substrate concentration. Bioresour. Technol. 100(2), 717-723.

Jarzębińska, A., Rowiński, P., Zawisza, I., Bilewicz, R., Siegfried, L. and Kaden, T. (1999) Modified electrode surfaces for catalytic reduction of carbon dioxide. Anal. Chim. Acta 396(1), 1-12.

Jeremiasse, A.W., Hamelers, H.V., Saakes, M. and Buisman, C.J. (2010) Ni foam cathode enables high volumetric $\mathrm{H} 2$ production in a microbial electrolysis cell. Int. J. Hydrogen Energy 35(23), 12716-12723.

Jiao, W., Luo, S., He, Z. and Liu, Y. (2016) Applications of high gravity technologies for wastewater treatment: A review. Chem. Eng. J.

Kelly, P.T. and He, Z. (2014) Nutrients removal and recovery in bioelectrochemical systems: a review. Bioresour. Technol. 153, 351-360.

Ki, D., Popat, S.C. and Torres, C.I. (2016) Reduced overpotentials in microbial electrolysis cells through improved design, operation, and electrochemical characterization. Chem. Eng. J. 287, 181-188.

Kim, J., Kim, K., Ye, H., Lee, E., Shin, C., McCarty, P.L. and Bae, J. (2010) Anaerobic fluidized bed membrane bioreactor for wastewater treatment. Environ. Sci. Technol. 45(2), 576-581.

Kim, J.R., Cheng, S., Oh, S.-E. and Logan, B.E. (2007a) Power generation using different cation, anion, and ultrafiltration membranes in microbial fuel cells. Environ. Sci. Technol. 41(3), 1004-1009. 
Kim, J.R., Cheng, S., Oh, S.E. and Logan, B.E. (2007b) Power generation using different cation, anion, and ultrafiltration membranes in microbial fuel cells. Environ. Sci. Technol. 41(3), 1004-1009.

Kim, J.R., Zuo, Y., Regan, J.M. and Logan, B.E. (2008) Analysis of ammonia loss mechanisms in microbial fuel cells treating animal wastewater. Biotechnol. Bioeng. 99(5), 1120-1127.

Klaysom, C., Cath, T.Y., Depuydt, T. and Vankelecom, I.F. (2013) Forward and pressure retarded osmosis: potential solutions for global challenges in energy and water supply. Chemical Society Reviews 42(16), 6959-6989.

Koelliker, J. and Kissel, D. (1988) Chemical equilibria affecting ammonia volatilization, pp. 3752.

Kulikowska, D. and Klimiuk, E. (2008) The effect of landfill age on municipal leachate composition. Bioresour. Technol. 99(13), 5981-5985.

Kumar, G., Saratale, R.G., Kadier, A., Sivagurunathan, P., Zhen, G., Kim, S.-H. and Saratale, G.D. (2017) A review on bio-electrochemical systems (BESs) for the syngas and value added biochemicals production. Chemosphere 177, 84-92.

Kundu, A., Sahu, J.N., Redzwan, G. and Hashim, M. (2013) An overview of cathode material and catalysts suitable for generating hydrogen in microbial electrolysis cell. Int. J. Hydrogen Energy 38(4), 1745-1757.

Kuntke, P., Geleji, M., Bruning, H., Zeeman, G., Hamelers, H. and Buisman, C. (2011) Effects of ammonium concentration and charge exchange on ammonium recovery from high strength wastewater using a microbial fuel cell. Bioresour. Technol. 102(6), 4376-4382.

Kuntke, P., Sleutels, T., Saakes, M. and Buisman, C. (2014) Hydrogen production and ammonium recovery from urine by a Microbial Electrolysis Cell. Int. J. Hydrogen Energy 39(10), 4771-4778.

Kuntke, P., Śmiech, K., Bruning, H., Zeeman, G., Saakes, M., Sleutels, T., Hamelers, H. and Buisman, C. (2012) Ammonium recovery and energy production from urine by a microbial fuel cell. Water Res. 46(8), 2627-2636.

Kurniawan, T.A., Lo, W.-h. and Chan, G.Y. (2006) Physico-chemical treatments for removal of recalcitrant contaminants from landfill leachate. J. Hazard. Mater. 129(1), 80-100.

Lackner, S., Gilbert, E.M., Vlaeminck, S.E., Joss, A., Horn, H. and van Loosdrecht, M.C. (2014) Full-scale partial nitritation/anammox experiences-an application survey. Water Res. 55, 292-303.

Ledezma, P., Kuntke, P., Buisman, C.J., Keller, J. and Freguia, S. (2015) Source-separated urine opens golden opportunities for microbial electrochemical technologies. Trends Biotechnol. 33(4), 214-220.

Lee, M.-Y., Kim, K.-Y., Yang, E. and Kim, I.S. (2015) Evaluation of hydrogen production and internal resistance in forward osmosis membrane integrated microbial electrolysis cells. Bioresour. Technol. 187, 106-112.

Lee, S., Boo, C., Elimelech, M. and Hong, S. (2010) Comparison of fouling behavior in forward osmosis (FO) and reverse osmosis (RO). J. Membr. Sci. 365(1), 34-39. 
Lefebvre, O., Tan, Z., Kharkwal, S. and Ng, H.Y. (2012) Effect of increasing anodic NaCl concentration on microbial fuel cell performance. Bioresour. Technol. 112, 336-340.

Lei, X., Sugiura, N., Feng, C. and Maekawa, T. (2007) Pretreatment of anaerobic digestion effluent with ammonia stripping and biogas purification. Journal of hazardous materials 145(3), 391-397.

Leong, J.X., Daud, W.R.W., Ghasemi, M., Liew, K.B. and Ismail, M. (2013) Ion exchange membranes as separators in microbial fuel cells for bioenergy conversion: a comprehensive review. Renewable and Sustainable Energy Reviews 28, 575-587.

Li, D., Zhang, X., Yao, J., Simon, G.P. and Wang, H. (2011a) Stimuli-responsive polymer hydrogels as a new class of draw agent for forward osmosis desalination. Chem. Commun. 47(6), 1710-1712.

Li, W.-W., Yu, H.-Q. and He, Z. (2014a) Towards sustainable wastewater treatment by using microbial fuel cells-centered technologies. Energ. Environ. Sci. 7(3), 911-924.

Li, W., Sheng, G., Liu, X. and Yu, H. (2011b) Recent advances in the separators for microbial fuel cells. Bioresour. Technol. 102(1), 244-252.

Li, W., Yu, H. and He, Z. (2014b) Towards sustainable wastewater treatment by using microbial fuel cells-centered technologies. Energ. Environ. Sci. 7(3), 911-924.

Li, X., Lu, Y. and He, Z. (2015) Removal of reverse-fluxed ammonium by anammox in a forward osmosis system using ammonium bicarbonate as a draw solute. J. Membr. Sci.

Liang, Z. and Liu, J. (2008) Landfill leachate treatment with a novel process: Anaerobic ammonium oxidation (Anammox) combined with soil infiltration system. J. Hazard. Mater. 151(1), 202-212.

Liao, P., Chen, A. and Lo, K. (1995) Removal of nitrogen from swine manure wastewaters by ammonia stripping. Bioresour. Technol. 54(1), 17-20.

Ling, M., Wang, K. and Chung, T.-S. (2010) Highly water-soluble magnetic nanoparticles as novel draw solutes in forward osmosis for water reuse. Ind. Eng. Chem. Res. 49(12), 5869-5876.

Liu, H., Grot, S. and Logan, B.E. (2005) Electrochemically assisted microbial production of hydrogen from acetate. Environ. Sci. Technol. 39(11), 4317-4320.

Liu, J., Liu, L., Gao, B. and Yang, F. (2013) Integration of bio-electrochemical cell in membrane bioreactor for membrane cathode fouling reduction through electricity generation. J. Membr. Sci. 430, 196-202.

Liu, J., Wang, X., Wang, Z., Lu, Y., Li, X. and Ren, Y. (2017) Integrating microbial fuel cells with anaerobic acidification and forward osmosis membrane for enhancing bio-electricity and water recovery from low-strength wastewater. Water Res. 110, 74-82.

Liu, Y., Qin, M., Luo, S., He, Z. and Qiao, R. (2016) Understanding Ammonium Transport in Bioelectrochemical Systems towards its Recovery. Sci. Rep. 6.

Logan, B.E. (2008) Microbial fuel cells, John Wiley \& Sons.

Logan, B.E. (2009) Exoelectrogenic bacteria that power microbial fuel cells. Nature reviews. Microbiology 7(5), 375. 
Logan, B.E., Call, D., Cheng, S., Hamelers, H.V., Sleutels, T.H., Jeremiasse, A.W. and Rozendal, R.A. (2008a) Microbial electrolysis cells for high yield hydrogen gas production from organic matter. Environ. Sci. Technol. 42(23), 8630-8640.

Logan, B.E., Call, D., Cheng, S., Hamelers, H.V.M., Sleutels, T.H.J.A., Jeremiasse, A.W. and Rozendal, R.A. (2008b) Microbial electrolysis cells for high yield hydrogen gas production from organic matter. Environ. Sci. Technol. 42(23), 8630-8640.

Logan, B.E. and Elimelech, M. (2012) Membrane-based processes for sustainable power generation using water. Nature 488(7411), 313-319.

Logan, B.E., Hamelers, B., Rozendal, R., Schröder, U., Keller, J., Freguia, S., Aelterman, P., Verstraete, W. and Rabaey, K. (2006) Microbial fuel cells: methodology and technology. Environ. Sci. Technol. 40(17), 5181-5192.

Logan, B.E. and Rabaey, K. (2012) Conversion of wastes into bioelectricity and chemicals by using microbial electrochemical technologies. Science 337(6095), 686-690.

Lu, X., Boo, C., Ma, J. and Elimelech, M. (2014a) Bidirectional diffusion of ammonium and sodium cations in forward osmosis: Role of membrane active layer surface chemistry and charge. Environ. Sci. Technol. 48(24), 14369-14376.

Lu, Y. and He, Z. (2015) Mitigation of Salinity Buildup and Recovery of Wasted Salts in a Hybrid Osmotic Membrane Bioreactor-Electrodialysis System. Environ. Sci. Technol. 49(17), 10529-10535.

Lu, Y., Qin, M., Yuan, H., Abu-Reesh, I.M. and He, Z. (2014b) When bioelectrochemical systems meet forward osmosis: accomplishing wastewater treatment and reuse through synergy. Water 7(1), 38-50.

Luo, S., Sun, H., Ping, Q., Jin, R. and He, Z. (2016) A review of modeling bioelectrochemical systems: engineering and statistical aspects. Energies 9(2), 111.

Lutchmiah, K., Verliefde, A., Roest, K., Rietveld, L. and Cornelissen, E. (2014a) Forward osmosis for application in wastewater treatment: A review. Water Res. 58, 179-197.

Lutchmiah, K., Verliefde, A., Roest, K., Rietveld, L.C. and Cornelissen, E.R. (2014b) Forward osmosis for application in wastewater treatment: a review. Water Res. 58, 179-197.

Mahmoud, M., Parameswaran, P., Torres, C.I. and Rittmann, B.E. (2014) Fermentation pretreatment of landfill leachate for enhanced electron recovery in a microbial electrolysis cell. Bioresour. Technol. 151, 151-158.

Manish, S. and Banerjee, R. (2008) Comparison of biohydrogen production processes. Int. J. Hydrogen Energy 33(1), 279-286.

Manuel, M.-F., Neburchilov, V., Wang, H., Guiot, S. and Tartakovsky, B. (2010) Hydrogen production in a microbial electrolysis cell with nickel-based gas diffusion cathodes. J. Power Sources 195(17), 5514-5519.

Matassa, S., Batstone, D.J., Hülsen, T., Schnoor, J. and Verstraete, W. (2015a) Can direct conversion of used nitrogen to new feed and protein help feed the world?, ACS Publications. 
Matassa, S., Batstone, D.J., Hülsen, T., Schnoor, J. and Verstraete, W. (2015b) Can direct conversion of used nitrogen to new feed and protein help feed the world? Environ. Sci. Technol. 49(9), 5247-5254.

Maurer, M., Schwegler, P. and Larsen, T. (2003a) Nutrients in urine: energetic aspects of removal and recovery. Water Science \& Technology 48(1), 37-46.

Maurer, M., Schwegler, P. and Larsen, T. (2003b) Nutrients in urine: energetic aspects of removal and recovery. Water Sci. Technol. 48(1), 37-46.

McCarty, P.L., Bae, J. and Kim, J. (2011) Domestic wastewater treatment as a net energy producer-can this be achieved? Environ. Sci. Technol. 45(17), 7100-7106.

McCutcheon, J.R., McGinnis, R.L. and Elimelech, M. (2005) A novel ammonia — carbon dioxide forward (direct) osmosis desalination process. Desalination 174(1), 1-11.

McCutcheon, J.R., McGinnis, R.L. and Elimelech, M. (2006) Desalination by ammonia-carbon dioxide forward osmosis: influence of draw and feed solution concentrations on process performance. J. Membr. Sci. 278(1), 114-123.

McGinnis, R.L., Hancock, N.T., Nowosielski-Slepowron, M.S. and McGurgan, G.D. (2013) Pilot demonstration of the $\mathrm{NH} 3 / \mathrm{CO} 2$ forward osmosis desalination process on high salinity brines. Desalination 312, 67-74.

McGinnis, R.L., McCutcheon, J.R. and Elimelech, M. (2007) A novel ammonia-carbon dioxide osmotic heat engine for power generation. J. Membr. Sci. 305(1), 13-19.

Mcintyre, N.S. and Cook, M.G. (1975) X-Ray Photoelectron Studies on Some Oxides and Hydroxides of Cobalt, Nickel, and Copper. Anal. Chem. 47(13), 2208-2213.

Oh, S.-E. and Logan, B.E. (2007) Voltage reversal during microbial fuel cell stack operation. J. Power Sources 167(1), 11-17.

Pant, D., Van Bogaert, G., Diels, L. and Vanbroekhoven, K. (2010) A review of the substrates used in microbial fuel cells (MFCs) for sustainable energy production. Bioresour. Technol. 101(6), 1533-1543.

Pardeshi, P. and Mungray, A. (2014) High flux layer by layer polyelectrolyte FO membrane: toward enhanced performance for osmotic microbial fuel cell. Int. J. Polym. Mater. Po. 63(12), 595-601.

Park, J., Jin, H., Lim, B.R., Park, K.Y. and Lee, K. (2010) Ammonia removal from anaerobic digestion effluent of livestock waste using green alga Scenedesmus sp. Bioresour. Technol. 101(22), 8649-8657.

Peng, S., Liang, D.-W., Diao, P., Liu, Y., Lan, F., Yang, Y., Lu, S. and Xiang, Y. (2013) Nernstping-pong model for evaluating the effects of the substrate concentration and anode potential on the kinetic characteristics of bioanode. Bioresour. Technol. 136, 610-616.

Persat, A., Chambers, R.D. and Santiago, J.G. (2009) Basic principles of electrolyte chemistry for microfluidic electrokinetics. Part I: acid-base equilibria and $\mathrm{pH}$ buffers. Lab on a Chip 9(17), 2437-2453.

Phillip, W.A., Yong, J.S. and Elimelech, M. (2010) Reverse draw solute permeation in forward osmosis: modeling and experiments. Environ. Sci. Technol. 44(13), 5170-5176. 
Phuntsho, S., Kim, J.E., Johir, M.A., Hong, S., Li, Z., Ghaffour, N., Leiknes, T. and Shon, H.K. (2016) Fertiliser drawn forward osmosis process: Pilot-scale desalination of mine impaired water for fertigation. J. Membr. Sci. 508, 22-31.

Phuntsho, S., Shon, H.K., Hong, S., Lee, S. and Vigneswaran, S. (2011) A novel low energy fertilizer driven forward osmosis desalination for direct fertigation: evaluating the performance of fertilizer draw solutions. J. Membr. Sci. 375(1), 172-181.

Phuntsho, S., Shon, H.K., Majeed, T., El Saliby, I., Vigneswaran, S., Kandasamy, J., Hong, S. and Lee, S. (2012) Blended fertilizers as draw solutions for fertilizer-drawn forward osmosis desalination. Environ. Sci. Technol. 46(8), 4567-4575.

Picioreanu, C., Head, I.M., Katuri, K.P., van Loosdrecht, M.C. and Scott, K. (2007) A computational model for biofilm-based microbial fuel cells. Water Res. 41(13), 2921-2940.

Ping, Q., Huang, Z., Dosoretz, C. and He, Z. (2015) Integrated experimental investigation and mathematical modeling of brackish water desalination and wastewater treatment in microbial desalination cells. Water Res. 77, 13-23.

Ping, Q., Porat, O., Dosoretz, C.G. and He, Z. (2016) Bioelectricity inhibits back diffusion from the anolyte into the desalinated stream in microbial desalination cells. Water Res. 88, 266273.

Ping, Q., Zhang, C., Chen, X., Zhang, B., Huang, Z. and He, Z. (2014) Mathematical model of dynamic behavior of microbial desalination cells for simultaneous wastewater treatment and water desalination. Environ. Sci. Technol. 48(21), 13010-13019.

Pinto, R., Srinivasan, B., Manuel, M.-F. and Tartakovsky, B. (2010) A two-population bioelectrochemical model of a microbial fuel cell. Bioresour. Technol. 101(14), 5256-5265.

Powers, S.E., Collins, A.G., Edzwald, J.K. and Dietrich, J.M. (1987) Modeling an aerated bubble ammonia stripping process. J. - Water Pollut. Control Fed. 59(2), 92-100.

Puig, S., Serra, M., Coma, M., Cabré, M., Balaguer, M.D. and Colprim, J. (2011) Microbial fuel cell application in landfill leachate treatment. J. Hazard. Mater. 185(2), 763-767.

Puyol, D., Batstone, D.J., Hülsen, T., Astals, S., Peces, M. and Krömer, J.O. (2016) Resource recovery from wastewater by biological technologies: opportunities, challenges, and prospects. Frontiers in microbiology 7.

Qin, M., Abu-Reesh, I.M. and He, Z. (2016a) Effects of current generation and electrolyte pH on reverse salt flux across thin film composite membrane in osmotic microbial fuel cells. Water Res. 105, 583-590.

Qin, M. and He, Z. (2014) Self-supplied ammonium bicarbonate draw solute for achieving wastewater treatment and recovery in a microbial electrolysis cell-forward osmosiscoupled system. Environ. Sci. Technol. Lett. 1(10), 437-441.

Qin, M., Hynes, E.A., Abu-Reesh, I.M. and He, Z. (2017) Ammonium removal from synthetic wastewater promoted by current generation and water flux in an osmotic microbial fuel cell. J Clean Prod. 149, 856-862. 
Qin, M., Molitor, H., Brazil, B., Novak, J.T. and He, Z. (2016b) Recovery of nitrogen and water from landfill leachate by a microbial electrolysis cell-forward osmosis system. Bioresour. Technol. 200, 485-492.

Qin, M., Ping, Q., Lu, Y., Abu-Reesh, I.M. and He, Z. (2015) Understanding electricity generation in osmotic microbial fuel cells through integrated experimental investigation and mathematical modeling. Bioresour. Technol. 195, 194-201.

Rabaey, K., Angenent, L., Schroder, U. and Keller, J. (2009) Bioelectrochemical systems, IWA publishing.

Rabaey, K. and Rozendal, R.A. (2010) Microbial electrosynthesis-revisiting the electrical route for microbial production. Nature Rev. Microbiol. 8(10), 706-716.

Rabaey, K. and Verstraete, W. (2005) Microbial fuel cells: novel biotechnology for energy generation. Trends Biotechnol. 23(6), 291-298.

Raghavulu, S.V., Mohan, S.V., Goud, R.K. and Sarma, P. (2009) Effect of anodic pH microenvironment on microbial fuel cell (MFC) performance in concurrence with aerated and ferricyanide catholytes. Electrochem. Commun. 11(2), 371-375.

Recio-Garrido, D., Perrier, M. and Tartakovsky, B. (2016) Modeling, optimization and control of bioelectrochemical systems. Chem. Eng. J. 289, 180-190.

Renou, S., Givaudan, J., Poulain, S., Dirassouyan, F. and Moulin, P. (2008) Landfill leachate treatment: review and opportunity. J. Hazard. Mater. 150(3), 468-493.

Rittmann, B.E. and McCarty, P.L. (2012) Environmental biotechnology: principles and applications, Tata McGraw-Hill Education.

Rousseau, R., Dominguez-Benetton, X., Délia, M.-L. and Bergel, A. (2013) Microbial bioanodes with high salinity tolerance for microbial fuel cells and microbial electrolysis cells. Electrochem. Commun. 33, 1-4.

Rozendal, R.A., Hamelers, H.V. and Buisman, C.J. (2006a) Effects of membrane cation transport on $\mathrm{pH}$ and microbial fuel cell performance. Environ. Sci. Technol. 40(17), 5206-5211.

Rozendal, R.A., Hamelers, H.V., Euverink, G.J., Metz, S.J. and Buisman, C.J. (2006b) Principle and perspectives of hydrogen production through biocatalyzed electrolysis. Int. J. Hydrogen Energy 31(12), 1632-1640.

Ruscalleda, M., López, H., Ganigué, R., Puig, S., Balaguer, M. and Colprim, J. (2008) Heterotrophic denitrification on granular anammox SBR treating urban landfill leachate. Water Sci. Technol 58(9), 1749-1755.

Sánchez, E., Milán, Z., Borja, R., Weiland, P. and Rodriguez, X. (1995) Piggery waste treatment by anaerobic digestion and nutrient removal by ionic exchange. Resources, conservation and recycling 15(3), 235-244.

Sarmidi, M.R. (2009) Recovery of sodium citrate and citric acid by one step electrodialysis process. Ph.D. Dissertation, Universiti Teknologi Malaysia, Johor ,Malaysia.

Sata, T. (2004) Ion exchange membranes: preparation, characterization, modification and application, Royal Society of chemistry. 
Schröder, U., Harnisch, F. and Angenent, L.T. (2015) Microbial electrochemistry and technology: terminology and classification. Energ. Environ. Sci. 8(2), 513-519.

Selembo, P.A., Merrill, M.D. and Logan, B.E. (2009) The use of stainless steel and nickel alloys as low-cost cathodes in microbial electrolysis cells. J. Power Sources 190(2), 271-278.

Selembo, P.A., Merrill, M.D. and Logan, B.E. (2010) Hydrogen production with nickel powder cathode catalysts in microbial electrolysis cells. Int. J. Hydrogen Energy 35(2), 428-437.

Shaffer, D.L., Werber, J.R., Jaramillo, H., Lin, S. and Elimelech, M. (2015) Forward osmosis: Where are we now? Desalination 356, 271-284.

Sharma, S.K. and Sanghi, R. (2013) Wastewater Reuse and Management, Springer.

She, Q., Jin, X., Li, Q. and Tang, C.Y. (2012) Relating reverse and forward solute diffusion to membrane fouling in osmotically driven membrane processes. Water Res. 46(7), 24782486.

Shouliang, H., Beidou, X., Haichan, Y., Liansheng, H., Shilei, F. and Hongliang, L. (2008) Characteristics of dissolved organic matter (DOM) in leachate with different landfill ages. Journal of Environmental Sciences 20(4), 492-498.

Singh, A., Chang, S.L.Y., Hocking, R.K., Bach, U. and Spiccia, L. (2013) Highly active nickel oxide water oxidation catalysts deposited from molecular complexes. Energ. Environ. Sci. 6(2), 579-586.

Sleutels, T.H., Hamelers, H.V., Rozendal, R.A. and Buisman, C.J. (2009) Ion transport resistance in microbial electrolysis cells with anion and cation exchange membranes. Int. J. Hydrogen Energy 34(9), 3612-3620.

Sprynskyy, M., Lebedynets, M., Zbytniewski, R., Namieśnik, J. and Buszewski, B. (2005) Ammonium removal from aqueous solution by natural zeolite, Transcarpathian mordenite, kinetics, equilibrium and column tests. Sep. Purif. Technol. 46(3), 155-160.

Stenina, I., Sistat, P., Rebrov, A., Pourcelly, G. and Yaroslavtsev, A. (2004) Ion mobility in Nafion-117 membranes. Desalination 170(1), 49-57.

Stillman, D., Krupp, L. and La, Y.-H. (2014) Mesh-reinforced thin film composite membranes for forward osmosis applications: the structure-performance relationship. J. Membr. Sci. 468, 308-316.

Tang, C.Y., Kwon, Y.-N. and Leckie, J.O. (2007) Characterization of humic acid fouled reverse osmosis and nanofiltration membranes by transmission electron microscopy and streaming potential measurements. Environ. Sci. Technol. 41(3), 942-949.

Tartakovsky, B., Manuel, M.-F., Wang, H. and Guiot, S. (2009) High rate membrane-less microbial electrolysis cell for continuous hydrogen production. Int. J. Hydrogen Energy 34(2), 672-677.

Teng, S.-X., Tong, Z.-H., Li, W.-W., Wang, S.-G., Sheng, G.-P., Shi, X.-Y., Liu, X.-W. and Yu, H.-Q. (2010) Electricity generation from mixed volatile fatty acids using microbial fuel cells. Appl. Microbiol. Biotechnol. 87(6), 2365-2372.

Thompson, N.A. and Nicoll, P.G. (2011) Forward osmosis desalination: a commercial reality, pp. 4-9. 
Trypuć, M. and Kiełkowska, U. (1998) Solubility in the NH4HCO3 + NaHCO3 + H2O System. Journal of Chemical \& Engineering Data 43(2), 201-204.

Verrecht, B., Judd, S., Guglielmi, G., Brepols, C. and Mulder, J. (2008) An aeration energy model for an immersed membrane bioreactor. Water Res. 42(19), 4761-4770.

Verstraete, W., Van de Caveye, P. and Diamantis, V. (2009) Maximum use of resources present in domestic "used water". Bioresour. Technol. 100(23), 5537-5545.

Voelcker, N.H., Alfonso, I. and Ghadiri, M.R. (2008) Catalyzed oxidative corrosion of porous silicon used as an optical transducer for ligand-receptor interactions. Chembiochem 9(11), 1776-1786.

Wang, H. and Ren, Z.J. (2013) A comprehensive review of microbial electrochemical systems as a platform technology. Biotechnol. Adv. 31(8), 1796-1807.

Wang, H. and Ren, Z.J. (2014) Bioelectrochemical metal recovery from wastewater: a review. Water Res. 66, 219-232.

Wang, K.Y., Ong, R.C. and Chung, T.-S. (2010a) Double-skinned forward osmosis membranes for reducing internal concentration polarization within the porous sublayer. Industrial \& Engineering Chemistry Research 49(10), 4824-4831.

Wang, K.Y., Ong, R.C. and Chung, T.-S. (2010b) Double-skinned forward osmosis membranes for reducing internal concentration polarization within the porous sublayer. Ind. Eng. Chem. Res. 49(10), 4824-4831.

Wang, R., Shi, L., Tang, C.Y., Chou, S., Qiu, C. and Fane, A.G. (2010c) Characterization of novel forward osmosis hollow fiber membranes. J. Membr. Sci. 355(1), 158-167.

Wang, Z., Gong, H., Zhang, Y., Liang, P. and Wang, K. (2017) Nitrogen recovery from lowstrength wastewater by combined membrane capacitive deionization (MCDI) and ion exchange (IE) process. Chem. Eng. J. 316, 1-6.

Water, U. (2014) The United Nations world water development report 2014: water and energy. United Nations, Paris.

Werner, C.M., Logan, B.E., Saikaly, P.E. and Amy, G.L. (2013) Wastewater treatment, energy recovery and desalination using a forward osmosis membrane in an air-cathode microbial osmotic fuel cell. J. Membr. Sci. 428, 116-122.

Wojtowicz, J.A. (2001) The carbonate system in swimming pool water. Journal of the Swimming Pool and Spa Industry 4(1), 54-59.

Won, S.G., Jeon, D.Y., Kwag, J.H., Kim, J.D. and Ra, C.S. (2015) Nitrogen Removal from Milking Center Wastewater via Simultaneous Nitrification and Denitrification Using a Biofilm Filtration Reactor. Asian Australas. J. Anim. Sci. 28(6), 896-902.

Wu, D., Wang, T., Huang, X., Dolfing, J. and Xie, B. (2015) Perspective of harnessing energy from landfill leachate via microbial fuel cells: novel biofuels and electrogenic physiologies. Appl. Microbiol. Biotechnol., 1-10.

Wu, S., Li, H., Zhou, X., Liang, P., Zhang, X., Jiang, Y. and Huang, X. (2016) A novel pilot-scale stacked microbial fuel cell for efficient electricity generation and wastewater treatment. Water Res. 98, 396-403. 
Wu, X. and Modin, O. (2013) Ammonium recovery from reject water combined with hydrogen production in a bioelectrochemical reactor. Bioresour. Technol. 146, 530-536.

Wu, Y., Yang, Q., Zeng, Q., Ngo, H.H., Guo, W. and Zhang, H. (2017) Enhanced low C/N nitrogen removal in an innovative microbial fuel cell (MFC) with electroconductivity aerated membrane (EAM) as biocathode. Chem. Eng. J. 316, 315-322.

Xiao, L., Wen, Z., Ci, S., Chen, J. and He, Z. (2012) Carbon/iron-based nanorod catalysts for hydrogen production in microbial electrolysis cells. Nano Energy 1(5), 751-756.

Xie, M., Nghiem, L.D., Price, W.E. and Elimelech, M. (2012) Comparison of the removal of hydrophobic trace organic contaminants by forward osmosis and reverse osmosis. Water Res. 46(8), 2683-2692.

Yang, E., Chae, K.-J., Alayande, A.B., Kim, K.-Y. and Kim, I.S. (2016a) Concurrent performance improvement and biofouling mitigation in osmotic microbial fuel cells using a silver nanoparticle-polydopamine coated forward osmosis membrane. J. Membr. Sci. 513, 217225.

Yang, E., Chae, K.J. and Kim, I.S. (2015a) Comparison of different semipermeable membranes for power generation and water flux in osmotic microbial fuel cells. J. Chem. Technol. Biotechnol. 91(8), 2305-2312.

Yang, E., Kim, K.-Y., Chae, K.-J., Lee, M.-Y. and Kim, I.S. (2016b) Evaluation of energy and water recovery in forward osmosis-bioelectrochemical hybrid system with cellulose triacetate and polyamide asymmetric membrane in different orientations. Desalin. Water. Treat. 57(16), 7406-7413.

Yang, E., Kim, K.Y., Chae, K.J., Lee, M.Y. and Kim, I.S. (2015b) Evaluation of energy and water recovery in forward osmosis-bioelectrochemical hybrid system with cellulose triacetate and polyamide asymmetric membrane in different orientations. Desalin. Water. Treat., 18.

Yang, Y., Li, X., Yang, X. and He, Z. (2016c) Membrane aeration as an energy-efficient method for supplying oxygen to microbial fuel cells. RSC Adv. 6(55), 49787-49791.

Yang, Y., Xu, M., Guo, J. and Sun, G. (2012) Bacterial extracellular electron transfer in bioelectrochemical systems. Process Biochem. 47(12), 1707-1714.

Yong, J.S., Phillip, W.A. and Elimelech, M. (2012) Reverse permeation of weak electrolyte draw solutes in forward osmosis. Industrial \& Engineering Chemistry Research 51(41), 13463 13472.

Yossan, S., Xiao, L., Prasertsan, P. and He, Z. (2013) Hydrogen production in microbial electrolysis cells: Choice of catholyte. Int. J. Hydrogen Energy 38(23), 9619-9624.

Yuan, H., Abu-Reesh, I.M. and He, Z. (2015a) Enhancing desalination and wastewater treatment by coupling microbial desalination cells with forward osmosis. Chem. Eng. J. 270, 437443.

Yuan, H., Abu-Reesh, I.M. and He, Z. (2016) Mathematical modeling assisted investigation of forward osmosis as pretreatment for microbial desalination cells to achieve continuous water desalination and wastewater treatment. J. Membr. Sci. 502, 116-123. 
Yuan, H. and He, Z. (2015) Integrating membrane filtration into bioelectrochemical systems as next generation energy-efficient wastewater treatment technologies for water reclamation: A review. Bioresour. Technol. 195, 202-209.

Yuan, H., Li, J., Yuan, C. and He, Z. (2014) Facile Synthesis of MoS2@ CNT as an Effective Catalyst for Hydrogen Production in Microbial Electrolysis Cells. ChemElectroChem $1(11), 1828-1833$.

Yuan, H., Lu, Y., Abu-Reesh, I.M. and He, Z. (2015b) Bioelectrochemical production of hydrogen in an innovative pressure-retarded osmosis/microbial electrolysis cell system: experiments and modeling. Biotechnol. Biofuels 8(1), 116.

Zang, G., Sheng, G., Li, W., Tong, Z., Zeng, R.J., Shi, C. and Yu, H. (2012) Nutrient removal and energy production in a urine treatment process using magnesium ammonium phosphate precipitation and a microbial fuel cell technique. Phys. Chem. Chem. Phys. 14(6), 19781984.

Zeng, Y., Choo, Y.F., Kim, B.-H. and Wu, P. (2010) Modelling and simulation of two-chamber microbial fuel cell. J. Power Sources 195(1), 79-89.

Zhalko-Titarenko, O., Lazurskii, O. and Pokhodenko, V. (1990) Electrocatalytic reduction of carbon dioxide on electrodes modified by polypyrrole with an immobilized complex of nickel with 1, 4, 8, 11-tetraazacyclotetradecane. Theor. Exp. Chem. 26(1), 40-44.

Zhang, B. and He, Z. (2012) Integrated salinity reduction and water recovery in an osmotic microbial desalination cell. RSC Adv. 2(8), 3265-3269.

Zhang, B. and He, Z. (2013a) Improving water desalination by hydraulically coupling an osmotic microbial fuel cell with a microbial desalination cell. J. Membr. Sci. 441, 18-24.

Zhang, F., Brastad, K.S. and He, Z. (2011a) Integrating forward osmosis into microbial fuel cells for wastewater treatment, water extraction and bioelectricity generation. Environ. Sci. Technol. 45(15), 6690-6696.

Zhang, F. and He, Z. (2013b) A cooperative microbial fuel cell system for waste treatment and energy recovery. Environmental technology 34(13-14), 1905-1913.

Zhang, F. and He, Z. (2015) Scaling up microbial desalination cell system with a post-aerobic process for simultaneous wastewater treatment and seawater desalination. Desalination 360, 28-34.

Zhang, F., Jacobson, K.S., Torres, P. and He, Z. (2010) Effects of anolyte recirculation rates and catholytes on electricity generation in a litre-scale upflow microbial fuel cell. Energy Environ. Sci. 3(9), 1347-1352.

Zhang, F., Li, J. and He, Z. (2014a) A new method for nutrients removal and recovery from wastewater using a bioelectrochemical system. Bioresour. Technol. 166, 630-634.

Zhang, F., Li, J. and He, Z. (2014b) A New Method for Nutrients Removal and Recovery from Wastewater Using a Bioelectrochemical System. Bioresource Technology.

Zhang, L., Li, C., Ding, L., Xu, K. and Ren, H. (2011b) Influences of initial pH on performance and anodic microbes of fed-batch microbial fuel cells. J. Chem. Technol. Biotechnol. 86(9), 1226-1232. 
Zhang, M., Zhang, M.T., Hou, C., Ke, Z.F. and Lu, T.B. (2014c) Homogeneous Electrocatalytic Water Oxidation at Neutral $\mathrm{pH}$ by a Robust Macrocyclic Nickel (II) Complex. Angew. Chem. Int. Ed. 53(48), 13042-13048.

Zhang, S., Wang, P., Fu, X. and Chung, T.-S. (2014d) Sustainable water recovery from oily wastewater via forward osmosis-membrane distillation (FO-MD). Water Res. 52, 112-121.

Zhang, Y. and Angelidaki, I. (2015a) Counteracting ammonia inhibition during anaerobic digestion by recovery using submersible microbial desalination cell. Biotechnol. Bioeng. 112(7), 1478-1482.

Zhang, Y. and Angelidaki, I. (2015b) Submersible microbial desalination cell for simultaneous ammonia recovery and electricity production from anaerobic reactors containing high levels of ammonia. Bioresour. Technol. 177, 233-239.

Zhao, F., Harnisch, F., Schröder, U., Scholz, F., Bogdanoff, P. and Herrmann, I. (2006) Challenges and constraints of using oxygen cathodes in microbial fuel cells. Environ. Sci. Technol. 40(17), 5193-5199.

Zhao, S., Zou, L. and Mulcahy, D. (2011) Effects of membrane orientation on process performance in forward osmosis applications. J. Membr. Sci. 382(1), 308-315.

Zhao, S., Zou, L., Tang, C.Y. and Mulcahy, D. (2012) Recent developments in forward osmosis: opportunities and challenges. J. Membr. Sci. 396, 1-21.

Zhu, H., Zhang, L., Wen, X. and Huang, X. (2012) Feasibility of applying forward osmosis to the simultaneous thickening, digestion, and direct dewatering of waste activated sludge. Bioresour. Technol. 113, 207-213.

Zhu, X., Zhang, F., Li, W., Li, J., Li, L., Yu, H., Huang, M. and Huang, T. (2016) Insights into enhanced current generation of an osmotic microbial fuel cell under membrane fouling condition. J. Membr. Sci. 504, 40-46.

Zhu, X., Zhang, F., Li, W., Liu, H., Wang, Y. and Huang, M. (2015) Forward osmosis membrane favors an improved proton flux and electricity generation in microbial fuel cells. Desalination 372, 26-31.

Zhuang, L., Zhou, S., Li, Y. and Yuan, Y. (2010) Enhanced performance of air-cathode twochamber microbial fuel cells with high-pH anode and low-pH cathode. Bioresour. Technol. 101(10), 3514-3519.

Zou, S. and He, Z. (2016) Enhancing wastewater reuse by forward osmosis with self-diluted commercial fertilizers as draw solutes. Water Res. 99, 235-243.

Zou, S., Qin, M., Moreau, Y. and He, Z. (2017) Nutrient-Energy-Water Recovery from Synthetic Sidestream Centrate Using a Microbial Electrolysis Cell - Forward Osmosis Hybrid System. J Clean Prod. 154, 16-25. 